\title{
MEMBERS OF THE SOCIETY
}

\author{
October 1, 1948
}

The following list includes the name of every person who is a member of the Society as of October 1, 1948.

So far as information is available the listings are made up as follows: (a) Surname. A dagger before a surname indicates life membership. (b) Initials, or the given name when there is but one, except that the first given name of every woman member is given in full. Parentheses enclosing any part of a name indicate that that part is not generally used in correspondence. (c) Official title or position. (d) Name and address of institution or organization with which item (c) is associated. (e) The address to be used for mail if this is different from (d). The name of the city and postal district number, where necessary, are not repeated if they are the same as in (d). (f) Following the regular listing, information re military service or temporary appointments is given.

Abbott, J. C. Asst. Prof., U. S. Naval Acad., Annapolis, Md. 87 Shipwright St. Abdelhay, Jose. Prof., Univ. of Brazil, Rio de Janeiro, Brazil.

Abdelhay, Maria Y. de M.N. (Mrs. Jose). Asst. Prof., Univ. of Brazil, Rio de Janeiro, Brazil. 10 Prudente de Morais St., Ipanema, Rio de Janeiro.

Abernethy, J. J. Asst. Prof., Houston Coll. for Negroes, Univ. of Houston, Houston 4, Tex. 2519 Nagle St.

Abramowitz, Dr. Milton. Math., Computation Lab., National Bur. of Standards, 150 Nassau St., New York 7, N.Y. 832 Ocean Ave., Brooklyn 26, N.Y.

Adams, C. R. Prof., Brown Univ., Providence 12, R.I. 60 Intervale Rd., Providence 6.

Adams, E. P. Prof. Emeritus, Princeton Univ., Princeton, N.J. Walpole, Mass.

Adams, Louise. Asst. Prof., High Point Coll., High Point, N.C.

Adams, Dr. Rachel B. (Mrs. C. R.). 60 Intervale Rd., Providence 6, R.I.

Adelman, Dr. D. M. 1315 2d Ave., Los Angeles 6, Calif.

Adkins, L. K. Prof., State Teachers Coll., LaCrosse, Wis.

Adkisson, V. W. Prof., Univ. of Arkansas, Fayetteville, Ark. 236 Buchanan St.

Adler, Claire F. (Mrs. E. H.) Asso. Prof., New York Univ., New York 3, N.Y. 189-21 Tioga Dr., St. Albans, N.Y.

Adler, F. T. Asst. Prof., Physics Dept., Univ. of Wisconsin, Madison 6, Wis.

Adney, J. E. Asst., Ohio State Univ., Columbus 10, Ohio. Box 3094, University Station.

Adshead, J. G. Asso. Prof., Dalhousie Univ., Halifax, N.S., Canada.

Agard, H. L. Prof., Williams Coll., Williamstown, Mass.

Agnew, Dr. Jeanne L. (Mrs. T. L.). College Ct. 18 E., Stillwater, Okla.

Agnew, R. P. Prof., Cornell Univ., Ithaca, N.Y. 112 White Hall.

Ahlfors, L. V. Prof., Harvard Univ., Cambridge 38, Mass. 52 Dunster St.

Aissen, M. I. Research Asst., Stanford Univ., Stanford University, Calif.

Akers, L. W. Asst. Prof., Central Missouri Coll., Warrensburg, Mo.

Akutowicz, Dr. E. J. Member, Inst. for Advanced Study, Princeton, N.J.

Alaoglu, Dr. Leon. Operations Analyst, Hqs. Strategic Air Command, Andrews Air Force Base, Washington 20, D.C. 1204 Mississippi Ave. S.E.

Albers, L. U. Asst. Prof., Western Reserve Univ., Cleveland 6, Ohio. 740 E. 105th St., Cleveland 8.

Albert, A. A. Prof., Univ. of Chicago, Chicago 37, Ill. Eckhart Hall.

Albert, G. E. Asso. Prof., Univ. of Tennessee, Knoxville 16, Tenn.

Albert, O. W. Prof., Univ. of Redlands, Redlands, Calif. 629 Buena Vista St.

Albert, R. G. Grad. Div. of Applied Math., Brown Univ., Providence 12, R.I. 54 Lauriston St., Providence 6.

Alder, Dr. H. L. Instr., Univ. of California, Berkeley 4, Calif. 854 Santa Barbara Rd., Berkeley 7.

Alexander, A. B. Asst. Prof., Ripon Coll., Ripon, Wis.

Alexander, H. W. Prof., Adrian Coll., Adrian, Mich. 
Alexander, J. W. Prof., Inst. for Advanced Study, Princeton, N.J. 29 Cleveland Lane.

Alger, P. L. Staff Asst. to Manager of Eng., General Elec. Co., 1 River Rd., Schenectady 5, N.Y. 1758 Wendell Ave., Schenectady 8.

Allan, R. E. Teaching Asst., Tulane Univ. of Louisiana, New Orleans 15, La. 818 6th St. W., Birmingham 4, Ala.

Allen, E. B. Prof., Rensselaer Polytech. Inst., Troy, N.Y. 4 Sheldon Ave.

Allen, E. S. Prof., Iowa State Coll. of Agric. and Mech. Arts, Ames, Iowa.

Allen, Florence E. Asst. Prof. Emeritus, Univ. of Wisconsin, Madison 6, Wis. 219 Lathrop St., Madison 5.

Allen, Harriet W. Asso. Prof., Physics Dept., Connecticut Coll., New London, Conn.

Allen, W. A. Staff member, Los Alamos Scientific Lab., Univ. of California, Los Alamos, N.M. 687-C 24th St.

Allen, W. R. Instr., Univ. of Illinois, Chicago 11, Ill. 1469 Carmen Ave., Chicago 40.

Allendoerfer, C. B. Prof., Haverford Coll., Haverford, Pa. Member, Inst. for Advanced Study, Princeton, N.J.

Alsberg, Julius. Chief, Chemicals Unit, Tech. Industrial Intelligence Div., Commerce Dept., Washington, D.C. 2120 16th St. N.W., Washington 9.

Alt, Dr. F. L. Deputy Chief, Computing Lab., Ballistic Research Labs., Aberdeen Proving Ground, Md.

Ambrose, Warren. Asst. Prof., Massachusetts Inst. of Tech., Cambridge 39, Mass.

Ames, D. B. Asso. Prof., Rensselaer Polytech. Inst., Troy, N.Y. 33 Kinloch Ave.

Amira, Dr. B. A. Lect., Hebrew Univ., Jerusalem.

Amundson, N. R. Asst. Prof., Univ. of Minnesota, Minneapolis 14, Minn.

Ananda-Rau, K. Prof., Presidency Coll., Madras, India.

Ancochea, Germán. Prof., Univ. of Madrid, Madrid, Spain.

Andersen, A. F. Prof., Tech. Univ. of Denmark, Copenhagen K, Denmark. Parkvaenget 28, Charlottenlund, Denmark.

Anderson, A. D. Asst., Univ. of Oregon, Eugene, Ore.

Anderson, A. T. Instr., Cooper Union, New York 3, N.Y.; Teacher, Sewanhaka High School, Floral Park, N.Y. P.O. Box 124, Floral Park.

Anderson, E. W. Prof., Iowa State Coll. of Agric. and Mech. Arts, Ames, Iowa.

Anderson, Florence R. Lect., Univ. of Southern California, Los Angeles 7, Calif. 4924 4th Ave., Los Angeles 43.

Anderson, P. H. Prof., Coll. of Business Admin., Loyola Univ., New Orleans 15, $\mathrm{La}$.

Anderson, Dr. R. D. Instr., Univ. of Pennsylvania, Philadelphia 4, Pa.

Anderson, R. E. Instr., North Illinois State Teachers Coll., De Kalb, Ill.

Anderson, R. Lucile. Asst. Prof., Hunter Coll., New York 21, N.Y.

Anderson, S. S. Instr., Psychology Dept., Ohio Univ., Athens, Ohio.

Anderson, T. W. Asst. Prof., Columbia Úniv., New Y̌ork 27, N.Y.

Anderson, William. Instr., Univ. of Rochester, Rochester 3, N.Y. 1793 Mt. Hope, Rochester 7.

Anderson, Dr. W. E. Retired. 112 E. Walnut St., Oxford, Ohio.

Andree, R. V. Acting Instr., Univ, of Wisconsin, Madison 6, Wis. North Hall.

Andrew, Dr. M. M. Staff Member, Operations Evaluation Group, Div. of Industrial Cooperation, Massachusetts Inst. of Tech., Cambridge 39, Mass. Room 3827 (CNO), Washington 25, D.C.

Andrews, F. C. Asso., Univ. of Washington, Seattle 5, Wash.

Andrews, J. J. Instr., St. Louis Univ., St. Louis 3, Mo. 155 S. Sappington Rd., Kirkwood 22, Mo.

Andrews, T. B. Math., National Advisory Com. for Aeronautics, Langley Aeronautical Lab., Hampton, Va. 207 Armstrong Dr.

Anthony, M. L. Armour Research Foundation, Illinois Inst. of Tech., Chicago 16, III. 5624 Maryland Ave., Chicago 37.

Apostle, H. G. Asso. Prof., Grinnell Coll., Grinnell, Iowa.

Apostol, Dr. T. M. Instr., Univ. of California, Berkeley 4, Calif. 1934 Blake St. 
Appuhn, w. E. F., Prof., St. Francis Coll., Brooklyn 2, N.Y.; Adj. Prof., Brooklyn Polytech. Inst., Brooklyn 2, N.Y. 548 State St., Brooklyn 17.

†Archibald, R. C. Prof. Emeritus, Brown Univ., Providence 12, R.I.

Archibald, R. G. Prof., Queens Coll., Flushing, N.Y.

Arden, D. N. Fellow, Purdue Univ., Lafayette, Ind.

Arena, F. J. Instr., North Dakota State Coll., Fargo, N.D. 1020 N. 14th St.

Arens, Richard. Asst. Prof., Univ. of California at Los Angeles, Los Angeles 24, Calif. 1117 N. Clark St., Los Angeles 46.

Arms, R. A. Prof., Gettysburg Coll., Gettysburg, Pa. 59 W. Lincoln Ave.

Armstrong, Beulah M. Asst. Prof., Univ. of Illinois, Urbana, I11. 364 Mathematics Bldg.

Arnoff, E. L. Asst., California Inst. of Tech., Pasadena 4, Calif.

Arnold, B. H. Asst. Prof., Oregon State Coll., Corvallis, Ore.

Arnold, Dr. H. A. Instr., Coll. of Agric., Univ. of California, Davis, Calif.

Arnold, H. C. Tech. Dir., Federal Enameling and Stamping Co., McKees Rocks, $\mathrm{Pa} .76$ Standish Blvd., Pittsburgh 16, $\mathrm{Pa}$.

Arnold, H. E. Prof., Wesleyan Univ., Middletown, Conn.

Arnold, H. L. Prof., Humboldt State Coll., Arcata, Calif. Box 82.

†Arnold, J. W. Eng., International Telephone and Telegraph Corp., 67 Broad St., New York 4, N.Y. 37 Washington Sq., New York 11.

Arnold, K. J. Asst. Prof., Univ. of Wisconsin, Madison 6, Wis. North Hall.

Arnoldy, Sister M. Nicholas. Prof., Marymount Coll., Salina, Kan.

Aroian, L. A. Asst. Prof., Hunter Coll., New York 21, N.Y. 247 Wadsworth Ave., New York 33.

Arrow, K. J. Asst. Prof., Economics Dept., Univ. of Chicago, Chicago 37, I11. Cowles Comm. for Research in Economics.

Arsove, M. G. Brown Univ., Providence 12, R.I. 445-A Elmgrove Ave., Providence 6.

Artin, Emil. Prof., Princeton Univ., Princeton, N.J. 7 Evelyn Pl.

Artin, Natascha J. (Mrs. Emil). Research Asst., Inst. for Math. and Mech., New York Univ., New York 3, N.Y.

Artzy, Dr. Rafael. Teacher, Galilee Secondary School, Tiberias, Israel.

Ascher, R. N. Columbia Univ., New York 27, N.Y. 310 W. 79th St., New York 24.

Ashburn, Andrew. Asso. Prof., Texas State Coll. for Women, Denton, Tex. Box 344, Main Office.

Askovitz, S. I. Univ. of Pennsylvania, Philadelphia 4, Pa. 932 N. Franklin St., Philadelphia 23.

Asprey, Winifred A. Asst. Prof., Vassar Coll., Poughkeepsie, N.Y.

Assadourian, Dr. Fred. Development Eng., Federal Telecommunication Labs., Inc., Nutley, N.J. 333 3d Ave., New York 10, N.Y.

Astrachan, Max. Prof., Antioch Coll., Yellow Springs, Ohio.

Atanasoff, Dr. J. V. Chief, Acoustics Div., Naval Ordnance Lab., White Oak, Silver Spring 19, Md. Bldg. 206.

Atchison, Dr. W. F. Instr., Univ. of Illinois, Urbana, I11. 356 Mathematics Bldg. Atkins, Ferrel. Instr., Univ. of Kentucky, Lexington 29, Ky.

Atkins, F. E. Instr., State Teachers Coll., California, Pa.

Atkins, H. P. Asst. Prof., Univ. of Rochester, Rochester 3, N.Y. River Campus. Aucoin, A. A. Prof., Univ. of Houston, Houston 4, Tex.

Auerbach, Leon. Instr., Gallaudet Coll., Washington 2, D.C.

Aulbach, Helmut. Instr., Syracuse Univ., Syracuse 10, N.Y

Aurora, Silvio. Columbia Univ., New York 27, N.Y. 30-58 83d St., Flushing, N.Y.

Avann, S. P. Asst. Prof., Univ. of Washington, Seattle 5, Wash. Philosophy Hall.

Ayer, Miriam C. Asst. Prof., Wellesley Coll., Wellesley 81, Mass.

Ayres, Frank. Prof., Dickinson Coll., Carlisle, Pa. 426 S. Pitt St.

Ayres, H. C. Prof., Jersey City Junior Coll., Jersey City, N.J.

Ayres, W. L. Dean, School of Science, Purdue Univ., Lafayette, Ind.

Babcock, R. W. Dean, School of Arts and Sciences, Kansas State Coll. of Agric. and Applied Science, Manhattan, Kan. 
Babcock, Wealthy. Asso. Prof., Univ. of Kansas, Lawrence, Kan.

Bachiller, T. R. Prof., Univ. of Madrid, Madrid, Spain. 2 Tormes St.

Bacon, H. M. Asso. Prof., Stanford Univ., Stanford University, Calif., P.O. Box 1144.

Bade, W. G. Univ. of California at Los Angeles, Los Angeles 24, Calif. 3525 7th Ave., San Diego 3, Calif.

Baer, Reinhold. Prof., Univ. of Illinois, Urbana, Ill. 363 Mathematics Bldg.

Baeumler, H. W. Instr., Univ. of Buffalo, Buffalo 14, N.Y.

Bagby, L. C. Prof., Lawrence Inst. of Tech., Detroit, Mich.; Industrial Eng., Technical Associates, 424 Book Bldg., Detroit 26. 62 Glendale Ave., Highland Park 3, Mich.

Bagemihl, Frederick. Asst. Prof., Univ. of Rochester, Rochester 3, N.Y.

Baidaff, B. I. Prof., Univ. of Buenos Aires, Buenos Aires, Argentina. 560 Mayo Ave.

Bailey, A. W. Instr., Casper Junior Coll., Casper, Wyo. Box 408.

Bailey, E. A. Dean, Registrar, and Prof., LaGrange Coll., LaGrange, Ga.

Bailey, H. W. Asso. Dean, Chicago Undergraduate Div., Univ. of Illinois, Chicago 11, Ill.

Bailey, R. P. Asso. Prof., U.S. Naval Acad., Annapolis, Md.

Baker, Dr. E. G. Member of Tech. Staff, American Bur. of Shipping, 45 Broad St., New York 4, N.Y. 41 Yantacaw P1., Nutley 10, N.J.

Baker, Frances E. Asso. Prof., Vassar Coll., Poughkeepsie, N.Y.

Baker, G. A. Asst. Prof., Math. Dept., and Asst. Stat., Experimental Station, Coll. of Agric., Univ, of California, Davis, Calif.

Bal, K. Y. 500 Riverside Dr., New York 27, N.Y.

Ball, Dr. R. W. Instr., Univ. of Washington, Seattle 5, Wash.

Ballantine, Dr. Constance R. (Mrs. J. P.). Asso., Univ. of Washington, Seattle 5, Wash. 1802 Ravenna Blvd.

Ballantine, J. P. Prof., Univ. of Washington, Seattle 5, Wash. 1802 Ravenna Blvd.

Ballou, D. H. Asso. Prof., Middlebury Coll., Middlebury, Vt. 27 Weybridge St.

Bamforth, F. R. Prof., Ohio State Univ., Columbus 10, Ohio. 64 S. Vine St., Westerville, Ohio.

Banhagel, E. W. Instr., Northwestern Univ., Evanston, I11. 2347 Clark Ave., Detroit 9, Mich.

Bankier, J. D. Asst. Prof., McMaster Univ., Hamilton, Ont., Canada.

Banks, G. B. Prof., Niagara Univ., Niagara University, N.Y.

Baños, Alfredo. Asso. Prof., Physics Dept., Univ. of California at Los Angeles, Los Angeles 24, Calif.

Barajas, Alberto. Dir., Faculty of Sciences, Univ. of Mexico, Mexico, D.F., Mexico.

Barankin, Dr. E. W. Asst. Prof., Stat. Lab., Univ. of California, Berkeley 4, Calif.

Barber, S. F. Asst. Prof., City Coll., New York 31, N.Y.

Bardell, R. H. Asso. Prof., Univ. of Wisconsin in Milwaukee, Milwaukee 3, Wis.

Bareis, Grace M. Asst. Prof. Emeritus, Ohio State Univ., Columbus 10, Ohio. 164 13th Ave., Columbus 1.

Bargmann, Valentine. Asso. Prof., Princeton Univ., Princeton, N.J.

Bari, Ruth A. (Mrs. Arthur). Instr., Univ. of Maryland, College Park, Md. 4902 Poe Ave., Baltimore 15, Md.

Barker, J. E. Math., Naval Proving Ground, Dahlgren, Va. Box 92.

Barlaz, Joshua. Asst. Prof., Rutgers Univ., New Brunswick, N.J.

Barnard, R. W. Asso. Prof., Univ. of Chicago, Chicago 37, I11. 320 Eckhart Hall.

Barnes, J. L. Prof., Engineering Dept., Univ. of California at Los Angeles, Los Angeles 24, Calif. 17411 Posetano Rd., Pacific Palisades, Calif.

Barnett, H. H. Teaching Asst., Univ. of Minnesota, Minneapolis 14, Minn. 1224 Delaware, Lawrence, Kan.

Barnett, I. A. Prof., Univ. of Cincinnati, Cincinnati 21, Ohio.

Barnett, Joseph. Asso. Prof., Oklahoma Agric. and Mech. Coll., Stillwater, Okla. 520 Maple Ave.

Baron, Louis. Asst. Prof., New York Univ., New York 3, N.Y. 
Barr, W. J. Project Head, Experiment, Inc., Box 1-T, Richmond 2, Va.

Barral-Souto, José. Dir., Inst. of Biometrics, and Prof., Univ. of Buenos Aires, Buenos Aires, Argentina. Córdoba 1459.

Barrow, D. F. Prof., Univ. of Georgia, Athens, Ga. 260 Cherokee Ave.

Bartels, R. C. F. Asst. Prof., Univ. of Michigan, Ann Arbor, Mich.

Barten, H. J. Asst. Eng., Armour Research Foundation, Illinois Inst. of Tech., Chicago 16, Ill.

Bartholomay, A. F. Instr., Rutgers Univ., New Brunswick, N.J.

Bartky, Walter. Dean, Div. of Physical Sciences, and Prof., Univ. of Chicago, Chicago 37, Ill. Eckhart Hall.

Bartnoff, Shepard. Teaching Fellow, Physics Dept., Massachusetts Inst. of Tech., Cambridge 39, Mass.

Barton, Helen. Prof., Woman's Coll., Univ. of North Carolina, Greensboro, N.C.

Basch, A. E. Prof. of General Mech., Tech. Univ. of Vienna, Vienna IV/50, Austria.

Basoco, M. A., Prof., Univ. of Nebraska, Lincoln 8, Neb. 210 Burnett Hall.

Bassford, H. R. Vice Pres. and Chief Actuary, Metropolitan Life Ins. Co., 1 Madison Ave., New York 10, N.Y.

Basye, R. E. Asst. Prof., Agric. and Mech. Coll. of Texas, College Station, Tex. Box 5734.

Batchelder, P. M. Asso. Prof., Univ. of Texas, Austin 12, Tex. 910 W. 22d St., Austin 21.

Bateman, Felice D. (Mrs. P. T.). Instr., New Jersey Coll. for Women, Rutgers Univ., New Brunswick, N.J. 345 Nassau St., Princeton, N.J.

Bateman, Dr. P. T. Member, Inst. for Advanced Study, Princeton, N.J. 345 Nassau St.

Bates, Grace E. Asst. Prof., Mt. Holyoke Coll., South Hadley, Mass.

Bates, O. K. Prof., St. Lawrence Univ., Canton, N.Y. 44 E. Main St.

$\dagger$ Battig, Leon. Instr., Extension Center, Univ. of Wisconsin, Sheboygan, Wis. 2535 Elizabeth St.

Battin, I. L. Asst. Prof., Brothers Coll., Drew Univ., Madison, N.J.

Bauer, P. S. Pres., New England Electronics Corp., Rear 78 Mt. Vernon St., Lynn, Mass.

Baumgart, J. K. Asst. Prof., Elmhurst Coll., Elmhurst, Ill. 1441 S. 13th Ave., Maywood, Ill.

Bausch, A. F. Instr., Williams Coll., Williamstown, Mass. 184 Main St.

Baxter, Ruby. Chm., Div. of Science and Math., Frances Shimer Coll., Mt. Carroll, I11.

Bazer, Jack. Columbia Univ., New York 27, N.Y. 644 E. 170th St., New York 56.

Beach, J. W. Asso. Prof., New Mexico School of Mines, Socorro, N.M. P.O. Box 99.

Beal, F. W. Prof., Univ. of Pennsylvania, Philadelphia 4, Pa. Bennett Hall.

Beale, F. S. Asst. Prof., Lehigh Univ., Bethlehem, Pa. 316 W. Packer Ave.

Beard, Helen P. Asst. Prof., Newcomb Memorial Coll., Tulane Univ. of Louisiana, New Orleans 18, La.

Bearman, J. E. Asst. Prof., Univ. of Minnesota, Minneapolis 14, Minn. 1414 N. Vincent Ave., Minneapolis 11.

Beatley, Ralph. Asso. Prof., Grad. School of Education, Harvard Univ., Cambridge 38, Mass.

Beatty, H. M. Asst. Prof., Ohio State Univ., Columbus 10, Ohio. 200 Tibet Rd., Columbus 2.

Beatty, Samuel. Prof., Univ. of Toronto, Toronto 5, Ont., Canada.

Beaty, Dr. Marjorie H. (Mrs. D. W.). 314 Canby St., Vermillion, S.D.

Beaumont, R. A. Asso. Prof., Univ. of Washington, Seattle 5, Wash.

Beberman, Max. Teachers Coll., Columbia Univ., New York 27, N.Y. 28 W. 706th St., Shanks Village, Orangeburg, N.Y.

Bechtolsheim, Lulu H. (Mrs.). Asst. Prof., Univ. of Redlands, Redlands, Calif. 330 Cajon St.

Beckenbach, E. F. Prof., Univ. of California at Los Angeles; Math., Inst. for Numerical Analysis, National Bur. of Standards, Univ. of California at Los Angeles, Los Angeles 24, Calif. 
Becker, H. W. 1214 N. 34th St., Omaha 3, Neb.

Becker, Leonard. 2629 7th Ave., Los Angeles 16, Calif.

Becker, Dr. Miriam F. 533 W. 112th St., New York 25, N.Y.

Beckman, F. S. Instr., Pratt Inst., Brooklyn 5, N.Y. 1419 Jesup Ave., New York 52, N.Y.

Becknell, G. G. Prof. Physics Dept., Univ. of Tampa, Tampa 6, Fla. 6900 Dixon Ave., Tampa 4.

Beckwith, Dr. Ethelwynn R. (Mrs. W. E.). Retired. 2827 N. Farwell Ave., Milwaukee 11 , Wis.

Beckwith, w. S. Asso. Prof., Univ. of Georgia, Athens, Ga. 731 Cobb St.

Beegle, B. L. Prof., Seattle Pacific Coll., Seattle 99, Wash.

Beeman, W. E. Instr., Agric. and Mech. Coll. of Texas, College Station, Tex. 703A E. 31st St., Bryan, Tex.

Beenken, May M. Asso. Prof., Immaculate Heart Coll., Los Angeles 27, Calif. 1906 Park Ave., Los Angeles 26.

Beer, F. P. Asso. Prof., Lehigh Univ., Bethlehem, Pa.

Beesley, E. M. Asso. Prof., Univ. of Nevada, Reno, Nev.

Begle, E. G. Asst. Prof., Yale Univ., New Haven 11, Conn. 216 Leet Oliver Memorial Hall.

Behmer, E. A. Highway Eng., Minnesota State Highway Dept., St. Paul, Minn. 32 Inner Dr., St. Paul 5.

Behrend, Dr. F. A. Lect., Univ. of Melbourne, Melbourne N.3, Victoria, Australia.

Beinert, R. L. Instr., Hobart Coll., Geneva, N.Y. Coxe Hall.

Belai, Dr. Louise G. Instr., Chemistry and Physics Dept., Our Lady of Cincinnati Coll., Cincinnati 6, Ohio. 102 Millville Ave., Hamilton, Ohio.

Belcher, Dr. D. R. Treas., American Telephone and Telegraph Co., 195 Broadway, New York 7, N.Y.

Belgodere, Paul. Research Attaché, National Center of Scientific Research, Paris 7, France. P.O. Box 1807.

Bell, Alice K. Asso. Prof., Fresno State Coll., Fresno 4, Calif.

Bell, Clifford. Asso. Prof., Univ. of California at Los Angeles, Los Angeles 24, Calif. 10514 Rochester Ave.

Bell, E. L. Univ. of Texas, Austin, Tex. 4349 W. 21st St., Chicago 23, I11.

Bell, E. T. Prof., California Inst. of Tech., Pasadena 4, Calif.

Bell, Dr. J. C. Research Eng., Battelle Memorial Inst., 505 King Ave., Columbus 1, Ohio.

Bell, J. H. Asst. Prof., Michigan State Coll., East Lansing, Mich. 625 Cherry Lane.

Bell, Dr. Janie L. (Mrs. J. C.). 370 Brevoort Rd., Columbus 2, Ohio.

Bell, P. O. Prof., Univ. of Kansas, Lawrence, Kan. 2112 Vermont St.

Bellamy, B. C. Civil Eng., Lamont, Wyo.

Bellman, Richard. Asso. Prof., Stanford Univ., Stanford University, Calif.

Belsky, Aurora. Instr., Polytech. Inst. of Brooklyn, Brooklyn 2, N.Y. 50 Park Ter. E., New York 34, N.Y.

Belzer, R. L. Research Analyst, Rand Corp., Douglas Aircraft Co., 3000 Ocean Park Blvd., Santa Monica, Calif. Dept. 9-970.

Benac, T. J. Asso. Prof., U.S. Naval Acad., Annapolis, Md.

Benedick, J. W. Instr., State Teachers Coll., Cortland, N.Y. 45 Homer Ave.

Benedict, Dr. D. L. Fellow in Physics, Cruft Lab., Harvard Univ., Cambridge 38, Mass.

Benjamin, Dr. Isaiah. 4860 Victoria Ave., Montreal, Que., Canada.

$\dagger$ Bennett, A. A. Prof., Brown Univ., Providence 12, R.I.

Bennett, C. A. Stat., Nucleonics Div., Hanford Works, General Elec. Co., Richland, Wash. 1428 Marshall Ave.

Bennett, H. C. Instr., Syracuse Univ., Syracuse 10, N.Y. 7 Terrace P1., Troy, N.Y.

Bennett, Theodore. Prof., Marietta Coll., Marietta, Ohio.

Benscoter, S. U. Aeronautical Eng. National Advisory Corn. for Aeronautics, Langley Field, Va. 1596 Corson St., Pasadena 4, Calif.

Benson, Leon. Instr., Lehigh Univ., Bethlehem, Pa. 313 Packer Hall.

Bentley, Dr. A. F. Retired. Paoli, Ind. 
Bentley, R. R. Univ. of Chicago, Chicago 37, I11. 7646 Greenview Ave., Chicago 26.

Benton, R. F. Teaching Fellow, California Inst. of Tech., Pasadena 4, Calif. 241 S. Holliston Ave., Pasadena 5.

Bercos, James. Asst., Univ. of Illinois, Urbana, Ill. 4808 Washington Blvd., Chicago 44, I11.

Berg, P. W. Inst. for Math. and Mech., New York Univ., New York 3, N.Y.

Berg, W. D. Asst. Prof., Kenyon Coll., Gambier, Ohio. Box 285.

Berger, Dr. Agnes H. (Mrs.). 10 Park Ave., New York 16, N.Y.

Bergman, Dr. Stefan. Lect., 'Harvard Univ., Cambridge 38, Mass. 201 Pierce Hall.

Bergmann, P. G. Asso. Prof., Physics Dept., Syracuse Univ., Syracuse 10, N.Y.

Berkeley, E. C. Consultant, Edmund C. Berkeley and Associates, 36 W. 11th St., New York 11, N.Y.

Berkowitz, Jerome. New York Univ., New York, N.Y. 31-32 89th St., Flushing, N.Y.

Bernard, R. R. Teaching Asst., Univ. of Virginia, Charlottesville, Va. Box 1412, University Station.

Bernardi, S. D. Instr., New York Univ., New York 53, N.Y.

Bernhart, Arthur. Asso. Prof., Univ. of Oklahoma, Norman, Okla. 13 Faculty Exch.

Bernstein, Arthur. Care of Sheets, Coal Run, Ohio.

Bernstein, B. A. Prof., Univ. of California, Berkeley 4, Calif. 2785 Shasta Rd., Berkeley 8.

Bernstein, Dorothy L. Asst. Prof., Univ. of Rochester, Rochester 3, N.Y.

Bernstein, Felix. Prof. Emeritus, New York Univ., New York, N.Y.; Lect., Triple Cities Coll., Syracuse Univ., Endicott, N.Y.

Bernstorf, Ella E. Retired. 1439 E. 4th Ave., Winfred, Kan.

Berry, A. C. Prof., Lawrence Coll., Appleton, Wis.

Berry, W. J. Retired. The Berrypatch, Box 298, Stony Brook, N.Y.

Bers, Lipman. Asso. Prof., Syracuse Univ., Syracuse 10, N.Y.

Besancon, R. M. Asst. Prof., Univ. of I1linois, Chicago 12, I11.

Beth, R. A. Prof., Physics Dept., Western Reserve Univ., Cleveland 6, Ohio.

Betz, E. E. Asso. Prof., U.S. Naval Acad., Annapolis, Md.

Betz, Herman. Asso. Prof., Univ. of Missouri, Columbia, Mo.

Betz, Dr. William. Math. Specialist, Public Schools of Rochester, Rochester, N.Y. 652 Melville St., Rochester 9.

Beveridge, H. R. Prof., Monmouth Coll., Monmouth, Ill. 1043 E. Detroit Ave.

Bibb, S. F. Asso. Prof., Illinois Inst. of Tech., Chicago 16, I11.

Bickel, R. J. Asst. Prof., Drexel Inst. of Tech., Philadelphia 4, Pa. 516 Upland Rd., Havertown, Pa.

Bickerstaff, T. A. Prof., Univ. of Mississippi, University, Miss. P.O. Box 262.

Biesele, F. C. Asso. Prof., Univ. of Utah, Salt Lake City 1, Utah.

Bigelow, J. H. Electronic Computer Project, Inst. for Advanced Study, Princeton, N.J.

Biggerstaff, J. S. Instr., Rensselaer Polytech. Inst., Troy, N.Y.

Billard, R. G. Teacher, Bristol High School, Bristol, Vt.

Bilo, S. J. Instr., Pennsylvania State Coll., State College, Pa. University Club.

Bing, R. H. Asst. Prof., Univ. of Wisconsin, Madison 6, Wis. North Hall 207.

Biot, M. A. Prof., Physics Dept., Brown Univ., Providence 12, R.I.

Birchby, w. N. Asst. Prof., California Inst. of Tech., Pasadena 4, Calif.

Birchenough, Harry. Prof., New York State Coll. for Teachers, Albany 3, N.Y.

Bird, M. T. Asst. Prof., San Jose State Coll., San Jose 14, Calif.

Birkhoff, Garrett. Prof., Harvard Univ., Cambridge 38, Mass. 45 Fayerweather St.

Birnbaum, Z. W. Asso. Prof., Univ. of Washington, Seattle 5, Wash.

Bishop, M. L. Asso. Prof., Brooklyn Coll., Brooklyn 10, N.Y.

Bisshopp, K. E. Research Eng., Armour Research Foundation, Illinois Inst. of Tech., Chicago 16, I11.

Bissinger, Dr. B. H. Vice Pres., George Gillis Shoe Corp., Fitchburg, Mass.

Black, A. H. Asso. Prof., Southern Illinois Univ., Carbondale, I1l. Apt. V-1, Ordnance Plant Veterans Housing Project, Carterville, Ill. 
Black, Florence. Asso. Prof., Univ. of Kansas, Lawrence, Kan.

Black, H. L. Asst. Prof., Michigan State Coll., East Lansing, Mich. 209 Cedar St.

Blackall, Dr. C. J. North Baltimore, Ohio.

Blackstock, May C. (Mrs. C. E.). Instr., Univ. of Tennessee, Knoxville 16, Tenn. Blackwell, D. H. Prof., Howard Univ., Washington 1, D.C.

Blair, Dewey. Univ. of Cincinnati, Cincinnati 21, Ohio. 6754 Britton Ave., Cincinnati 27.

Blake, Dr. Archie. Stat., Office of the Army Surgeon General, Pentagon Bldg., Washington 25, D.C. 223 N. George Mason Dr., Arlington, Va.

Blake, Dr. E. M. Retired. Box 210, Mt. Kisco, N.Y.

Blake, R. G. Instr., Univ. of Florida, Gainesville, Fla. Box 25, R.F.D. 5.

Blakers, Dr. A. L. Instr., Princeton Univ., Princeton, N.J. Fine Hall.

Blanc, Charles. Prof., Univ. of Lausanne, Lausanne, Switzerland. 28 Montolivet St.

Blanch, Dr. Gertrude. Senior Math., Inst. for Numerical Analysis, National Bur. of Standards, Univ. of California at Los Angeles, Los Angeles 24, Calif.

Blanche, Dr. E. E. Prin. Admin. Analyst, Management Branch, Plans and Policy Office, Service, Supply and Procurement Div., Army Dept., Washington 25, D.C.; Lect., American Univ., Washington 16. 9409 Montgomery Ave., Chevy Chase 15, Md.

Blanco, B. C. Eng., General Elec. Co., 1 River Rd., Schenectady 5, N.Y. 13 State St.

Blanco, Enrique. Prof., Economics Dept., Univ. of Madrid, Madrid, Spain. 4 Nervión.

Blank, A. A. New York Univ., New York 3, N.Y. 2458 84th St., Brooklyn 14, N.Y.

Blankinship, W. A. Lt. (jg), U.S.N. Fine Hall, Princeton Univ., Princeton, N.J.

Blaquier, Juan. Prof., Univ. of Buenos Aires, Buenos Aires, Argentina. Arroyo 1000.

Blau, Dr. J. H. Instr., Massachusetts Inst. of Tech., Cambridge 39, Mass. 1377 Commonwealth Ave., Allston 34, Mass.

Bleick, W. E. Asso. Prof., U.S. Naval Postgrad. School, Annapolis, Md. 8 Taney Ave.

†Bliss, G. A. Prof. Emeritus, Univ. of Chicago, Chicago 37, Ill. Flossmoor, Ill.

Block, Daniel. Instr., Yeshiva Univ., New York 33, N.Y. 775 Garden St., New York 60

Block, H. D. Instr., Iowa State Coll. of Agric. and Mech. Arts, Ames, Iowa.

Block, R. B. Math., Air Forces, Wright Field, Ohio. 1933 N. Main St., Dayton 5, Ohio.

Blondeau, Maude B. (Mrs. E. E.). Instr., Univ. of Arkansas, Fayetteville, Ark. $651 \mathrm{~N}$. Storer St.

Bloom, G. M. Instr., Northwestern Univ., Evanston, Il1.

Blum, E. K. Lect., Columbia Univ., New York 27, N.Y. 699 Ocean Ave., Brooklyn 26, N.Y.

Blum, Joseph. Research Analyst, Army Dept., Washington 25, D.C. $1500 \mathrm{Key}$ Blvd., Arlington, Va.

Blumberg, Henry. Prof., Ohio State Univ., Columbus 10, Ohio. 76 E. Blake Ave., Columbus 2.

Blumenthal, L. M. Prof., Univ. of Missouri, Columbia, Mo.

Blyth, M. Isobel. Instr., Michigan State Coll., East Lansing, Mich.

Boas, Dr. Mary L. (Mrs. R. P.). 21 Chauncy St., Cambridge 38, Mass.

Boas, Dr. R. P. Exeeutive Editor, Mathematical Reviews, American Math. Society, Brown Univ., Providence 12, R.I. 21 Chauncy St., Cambridge 38, Mass.

Bobonis, Augusto. Dir., City Coll., Hato Rey, Puerto Rico. Box 9.

Bocanegra-Saldafia, Olga. Asst. Prof., Univ. of Puerto Rico, Rio Piedras, Puerto Rico. International House, Univ. of California, Berkeley 4, Calif.

Bochner, Salomon. Prof., Princeton Univ., Princeton, N.J. Fine Hall.

Boddy, Marie L. Asst., Univ. of Illinois, Urbana, Ill. 507 W. Green.

Bode, Dr. H. W. Research Math., Bell Telephone Labs., Murray Hill, N.J.

Boeder, Dr. Paul. Dir., Bur. of Visual Science, American Optical Co., Southbridge, Mass. 
Boehm, Frank. Manager, Life Ins. Dept., 80 Maiden Lane, New York 7, N.Y. Boeker, Dr. Mary D. (Mrs. G. F.). Instr., Brooklyn Coll., Brooklyn 10, N.Y. 37 Washington Sq. W., New York 11, N.Y.

Bogdanoff, J. L. Instr., Civil Eng. Dept., Columbia Univ., New York 27, N.Y. 123 N. Middletown Rd., Pearl River, N.Y.

Boggs, Dr. Elizabeth M. (Mrs. F. W.). 75 Edgemont Rd., Upper Montclair, N.J. Bohnenblust, H. F. Prof., California Inst. of Tech., Pasadena 4, Calif.

Bohr, H. A. Prof., Univ. of Copenhagen, Copenhagen $\varnothing$, Denmark. Regensen, St. Kannikestraede 2, Copenhagen $\mathrm{K}$.

Boldyreff, A. W. Asso. Prof., Univ. of New Mexico, Albuquerque, N.M.

Bolton, Grace L. Instr., Barnard Coll., Columbia Univ., New York 27, N.Y.

Bompiani, Enrico. Prof., Univ. of Rome, Rome, Italy. 22 Verona Way.

Booth, Dr. A. D. Dir., Electronic Computer Project, Birkbeck Coll., Univ. of London, London, England. The Cottage, Hampton-on-the-Hill, Warwick, England.

Boothby, W. M. Instr., Northwestern Univ., Evanston, Ill.

Bordner, C. L. Asso. Prof., Pennsylvania Military Coll., Chester, Pa. 329 W. Main St., Kutztown, Pa.

Borgers, Dr. A. M. F. Mandatory, Belgium National Fund for Scientific Research. Fellow, Princeton Univ. and Inst. for Advanced Study, Princeton, N.J. Graduate College.

Borgman, W. M. Asst. Dean of Admin., Wayne Univ., Detroit 1, Mich.

Borofsky, Samuel. Asst. Prof., Brooklyn Coll., Brooklyn 10, N.Y. 335 State St., Brooklyn 2.

Bortle, F. E. Asst. Prof., Iowa State Coll. of Agric. and Mech. Arts, Ames, Iowa.

Borůvka, Otakar. Prof., Masaryk Univ., Brünn, Czechoslovakia. 2 Kotlářská.

Boschan, Dr. Paul. Chief Analyst, Econometric Inst., Inc., 500 5th Ave., New York 18, N.Y. 104 W. 40th St.

Boswell, J. M. Pres., Cumberland Coll., Williamsburg, Ky.

Bosworth, F. D. Instr., Clark School, Hanover, N.H. 20 South St., Middlebury, $\mathrm{Vt}$.

Bothwell, Dr. Frank. Senior Math., Ordnance Research, Univ. of Chicago, Chicago 37, I11. Museum of Science and Industry.

Botsford, J. L. Asst. Prof., San Jose State Coll., San Jose, Calif.

Bott, George. Staff Member, Operations Evaluation Group, Div. of Industrial Cooperation, Massachusetts Inst. of Tech., Cambridge 39, Mass. Rm. 3827 (CNO), Navy Dept., Washington 25, D.C.

Botts, T. A. Acting Asst. Prof., Univ. of Virginia, Charlottesville, Va. 1567 University Ave.

Bourgin, D. G. Inst. for Advanced Study, Princeton, N.J.

Bourne, S. G. Instr., Johns Hopkins Univ., Baltimore 18, Md.

Bouwkamp, Dr. C. J. N. V. Philips' Lab., Gloeilampenfabrieken, Eindhoven, Netherlands. 10 Goorstraat.

Bowden, Joseph. Prof. Emeritus, Adelphi Coll., Garden City, N.Y. 21 Carleton P1., Baldwin, N.Y.

Bowden, Dr. Muriel. Brooklyn Coll., Brooklyn 10, N.Y. 405 Park Ave., New York 22, N.Y.

Bower, Julia W. Asso. Prof., Connecticut Coll., New London, Conn.

Bower, O. K. Asst. Prof., Úniv. of Illinois, Ürbana, Ill. 369 Mathematics Bldg.

Bowie, H. E. Asso. Prof., American International Coll., Springfield, Mass. 47 Midway St., Indian Orchard, Mass.

Bowker, A. H. Asst. Prof., Stanford Univ., Stanford University, Calif.

Bowker, J. G. Prof., Middlebury Coll., Middlebury, Vt. 14 Adirondack View.

Boyce, Jessie W. Prof., Nebraska State Teachers Coll., Wayne, Neb. 518 Lincoln St.

Boyce, M. G. Asso. Prof., Vanderbilt Univ., Nashville 4, Tenn. Box 152.

Boyd, Evelyn. Yale Univ., New Haven 11, Conn. 370 Temple St.

Boyd, P. P. Dean Emeritus and Prof. Emeritus, Univ. of Kentucky, Lexington 29, Ky. 119 Waller Ave., Lexington 2.

Boyer, C. B. Asso. Prof., Brooklyn Coll., Brooklyn 10, N.Y.

Boyer, Jean M. Instr., Univ. of Maryland, College Park, Md. 
Bradfield, G. F. Instr., Lake View High School, Chicago, I11. 1864 Sherman Ave., Evanston, I11.

Bradford, Thelma E. Asst. Prof., Delaware State Coll., Dover, Del. Box 31.

Bradford, W. H. Asso. Prof., John McNeese Junior Coll., Louisiana State Univ. and Agric. and Mech. Coll., Lake Charles, La.

Bradley, A. D. Asst. Prof., Hunter Coll., New York 21, N.Y. 66 Villard Ave., Hastings on Hudson 6, N.Y.

Bradley, Helen J. Instr., Univ. of Tennessee, Knoxville 16, Tenn.

Bradshaw, J. W. Prof. Emeritus, Univ. of Michigan, Ann Arbor, Mich. 1304 Cambridge Rd.

Brady, C. P. Asso. Prof., U.S. Naval Acad., Annapolis, Md.

Brady, G. V. Actuary, Metropolitan Life Ins. Co., 1 Madison Ave., New York 10, N.Y.

Brady, R. P. Instr., Illinois Inst. of Tech., Chicago 16, Ill. 5810 Harper Ave., Chicago 37.

Brady, W. G. Instr., Univ. of Wyoming, Laramie, Wyo.

Brahana, H. R. Prof., Univ. of Illinois, Urbana, Ill. 504 W. Nevada St.

Brainard, Millar. 114 Mt. Vernon St., Boston 8, Mass.

Brainerd, J. G. Prof., Moore School of Elec. Eng., Univ. of Pennsylvania, Philadelphia 4, $\mathrm{Pa}$.

Bramble, Dr. C. C. Dir., Computation and Ballistics Dept., Naval Proving Ground, Dahlgren, Va.

Brand, Louis. Prof., Univ. of Cincinnati, Cincinnati 21, Ohio. Visiting Prof., Univ. of Hawaii, Honolulu 10, T.H.

Brandt, Angeline J. Asso. Prof., Wheaton Coll., Wheaton, I11.

Brannon, Mildred J. Asst. Prof., Michigan State Normal Coll., Ypsilanti, Mich.

Branson, H. R. Prof., Physics Dept., Howard Univ., Washington 1, D.C.

Brant, Dr. Laura. 33 Capwell Ave., West Warwick, R.I.

Brauer, A. T. Prof., Univ. of North Carolina, Chapel Hill, N.C. 410 Patterson P1.

Brauer, Richard. Prof., Univ. of Michigan, Ann Arbor, Mich.

Braun, Hel. Prof., Göttingen Univ., Göttingen, Germany.

Bray, H. E. Prof., Rice Inst., Houston 1, Tex.

Breakwell, Dr. J. V. Instr., Tufts Coll., Medford 55, Mass.

Breit, Gregory. Prof., Physics Dept., Yale Univ., New Haven 11, Conn. Sloane Physics Lab.

Brelot, M. E. Prof., Univ. of Grenoble, Grenoble, France.

Brendel, Ruth A. Instr., Univ. of Buffalo, Buffalo 14, N.Y. 145 E. Morris Ave.

Brenke, W. C. Prof. Emeritus, Univ. of Nebraska, Lincoln 8, Neb. 1250 S. 21st St., Lincoln 2.

Brennan, J. F. Eng., Pacific Gas and Elec. Co., San Francisco, Calif. 1769 Broadway, San Francisco" 9.

Brenner, Clifford. Instr., Temple Univ., Philadelphia 22, Pa.

Brenner, J. L. Asst. Prof., Santa Barbara Coll., Univ. of California, Santa Barbara, Calif. Box 593, Goleta, Calif.

Breusch, R. H. Asso. Prof., Amherst Coll., Amherst, Mass. 12 Walnut St.

Breves Filho, J. A. Prof., Polytech. School, Univ. of Brazil, São Paulo, Brazil.

Brewer, B. W. Asst. Prof., Oregon State Coll., Corvallis, Ore. 17 S. Bluff St., Janesville, Wis.

Briant, Dr. R. C. Senior Fellow, Mellon Inst. of Industrial Research, Pittsburgh 13, Pa. 154 Gordon St., Pittsburgh 18.

Bridgman, P. W. Prof., Harvard Univ., Cambridge 38, Mass. Research Physics Lab.

Brigham, N. A. Asst. Prof., Univ. of Maryland, College Park, Md.

Bright, H. F. Registrar, San Angelo Coll., San Angelo, Tex. 1607 Shafter St.

Bright, S. K. Instr., Vanderbilt Univ., Nashville 4, Tenn.

Brink, R. W. Prof., Univ. of Minnesota, Minneapolis 14, Minn. 2243 Hoyt Ave. St. Paul 8, Minn.

Brinkmann, H. W. Prof., Swarthmore Coll., Swarthmore, Pa.

Bristow, Leonard. Asst. Prof., Univ. of Wyoming, Laramie, Wyo. 
Britten, Kathleen H. King's Coll., Univ. of London, London W.C. 2, England. 58 Driffold, Sutton Coldfield, Warwicks, England.

Britton, F. R. Lect., McMaster Univ., Hamilton, Ont., Canada. 184 Herkimer St. Britton, J. R. Asso. Prof., Univ. of Colorado, Boulder, Colo.

Brixey, J. C. Prof., Univ. of Oklahoma, Norman, Okla. 927 S. Pickard.

Brock, Paul. Math., Reeves Instrument Co., Inc., 215 E. 91 st St., New York 28, N.Y. 455 E. 14th St., New York 3.

Brodetsky, Selig. Prof., Univ. of Leeds, Leeds 2, England. Brotherton Lib.

Brooks, F. (L.). Prof., Kent State Univ., Kent, Ohio.

Brothers, W. H. Prof., Talladega Coll., Talladega, Ala.

Broussard, R. L. Louisiana State Univ. and Agric. and Mech. Coll., Baton Rouge, La. P.O. Box 8364.

Brown, Azelle. New York Univ., New York, N.Y. 84-19 Kent Rd., Jamaica 3, N.Y.

Brown, Dr. A. A. Deputy Dir., Operations Evaluation Group, Div. of Industrial Cooperation, Massachusetts Inst. of Tech., Cambridge 39, Mass. 8 Tauxemont Rd., Alexandria, Va.

Brown, A. B. Asso. Prof., Queens Coll., Flushing, N.Y.

Brown, Bailey. Prof., Amherst Coll., Amherst, Mass. 19 Hitchcock Rd.

Brown, B. H. Prof., Dartmouth Coll., Hanover, N.H. 7 Ripley Rd.

Brown, Dr. D. M. Supervisor, Applied Math. Group, Aeronautical Research Center, Univ. of Michigan, Ann Arbor, Mich. 519 Soule Blvd.

Brown, Rev. F. L. Instr., Univ. of Notre Dame, Notre Dame, Ind. 244 Dillon Hall.

Brown, Dr. F. W. Research Specialist, North American Aviation, Inc., Municipal Airport, Los Angeles 45, Calif. 8300 Fordham Rd.

Brown, G. W. Prof., Iowa State Coll. of Agric. and Mech. Arts, Ames, Iowa. Statistical Lab.

Brown, H. K. Asst. Prof., Mech. Eng. Dept., Northeastern Univ., Boston 15, Mass. 87 Harvard Ave., Brookline 46, Mass.

Brown, Dr. L. F. Consulting Physicist and Eng., 1236 Greenacre Ave., Hollywood 46, Calif.

Brown, Myrtle C. Asso. Prof., North Texas State Teachers Coll., Denton, Tex. 5334 Teachers College Station.

Brown, O. E. Prof., Case Inst. of Tech., Cleveland 6, Ohio. 1387 East Blvd.

Brown, R. G. Staff Member, Operations Evaluation Group, Navy Dept., Washington 25, D.C. Room 3827.

Brown, R. H. Lect., Columbia Univ., New York 27, N.Y. 1310 John Jay Hall.

Brown, T. H. Prof., Grad. School of Business Admin., Harvard Univ., Boston 63, Mass. 25 Meadow Way, Cambridge 38, Mass.

Browne, E. T. Prof., Univ. of North Carolina, Chapel Hill, N.C. 730 E. Franklin St.

Browne, Marjorie L. Teaching Fellow, Univ. of Michigan, Ann Arbor, Mich. $210 \mathrm{~W}$. Kingsley St.

Bruce, R. E. Prof. Emeritus, Boston Univ., Boston 16, Mass.

Bruck, R. H. Asso. Prof., Univ. of Wisconsin, Madison 6, Wis. North Hall.

Brudno, Charlotte. Research Asst., Theoretical Mech. Subdiv., Naval Ordnance Lab., White Oak, Silver Spring 19, Md. Bldg. 90.

Brunk, H. D. Asst. Prof., Rice Inst., Houston 1, Tex.

Bruns, W. J. Visiting Prof., Syracuse Univ., Syracuse 10, N.Y. 2605 Midland Ave., Syracuse 5.

Bruton, G. S. Prof., Univ. of the South, Sewanee, Tenn.

Bryan, N. R. Asso. Prof., Univ. of Georgia, Athens, Ga.

Bryan, W. S. Univ. of Washington, Seattle 5, Wash. 512 N. 83d St., Seattle 3.

Buch, K. R. Lect., Tech. Univ. of Denmark, Copenhagen K, Denmark. 14A Ejgaardsvej, Charlottenlund, Denmark.

Buchanan, Dr. Daniel. Retired. Faculty of Arts and Science, Univ. of British Columbia, Vancouver, B.C., Canada.

Buchanan, H. E. Prof. Emeritus, Tulane Univ. of Louisiana, New Orleans 15, La.

Buck, Ellen F. (Mrs. R. C.). Instr., Brown Univ., Providence 12, R.I. 31 Ontario St., Providence 7. 
Buck, Helen J. (Mrs. J. G.). Instr., Extension Center, Indiana Univ., South Bend 3, Ind. $107 \mathrm{~S}$. Hill St., South Bend 17.

Buck, R. C. Asst. Prof., Brown Univ., Providence 12, R.I. 31 Ontario St., Providence 7.

Buck, Thomas. Prof., Univ. of California, Berkeley 4, Calif. Faculty Club.

Buell, Dr. E. L. Math., Aerial Measurements Lab., Northwestern Tech. Inst., Evanston, Ill.

Bula, Dr. Clotilde A. 626 San Lorenzo, Olivos, F.C.C.A., Argentina.

Bullard, J. A. Prof., Univ. of Vermont and State Agric. Coll., Burlington, Vt. 110 Summit St.

Bullitt, W. M. Lawyer, Bullitt, Dawson and Tarrant, Kentucky Home Life Bldg., Louisville 2, Ky.

Bullock, R. C. Asso. Prof., State Coll. of Agric. and Eng., Univ. of North Carolina, Raleigh, N.C. Box 5548, State College Station.

Bumer, C. T. Prof., Clark Univ., Worcester, Mass. 43 Canterbury Rd., Winchester, Mass.

Bundgaard, S. B. E. Lect., Univ. of Copenhagen, Copenhagen $\varnothing$, Denmark. Bernstorffs vej 144, Hellerup, Denmark.

Bunyan, L. H. Asso. Prof., Rutgers Univ., New Brunswick, N.J.

Burcham, P. B. Asst. Prof., Univ. of Missouri, Columbia, Mo.

Burdette, A. C. Asst. Prof., Coll. of Agric., Univ. of California, Davis, Calif.

Bures, Dr. C. E. Instr., Philosophy Dept., Univ. of Oregon, Eugene, Ore.

Burgess, Louis. Attorney, 233 Broadway, New York 7, N.Y.

Burington, Dr. R. S. Chief Math. and Dir., Evaluation and Analysis Groups, Research and Development Div., Bur. of Ordnance, Navy Dept., Washington 25, D.C. 5200 N. Carlin Spring Rd., Arlington, Va.

Burk, J. D. Asso. Prof., Univ. of Toronto, Toronto 5, Ont., Canada.

Burke, Rev. J. C. Asst. Prof., Univ. of Notre Dame, Notre Dame, Ind. 201 St. Edward's Hall.

Burke, Sister M. Leonarda. Prof., Regis Coll., Weston 93, Mass.

Burkett, F. J. H. Asso. Prof., Union Coll., Schenectady 8, N.Y.

Burkill, Dr. J. C. Fellow and Lect., Cambridge Univ., Cambridge, England. 20 Chaucer Rd.

Burnam, J. E. Prof., Hardin-Simmons Univ., Abilene, Tex. 1141 Grape St.

Burns, G. P. Asst. Prof., Physics Dept., Mary Washington Coll., Univ. of Virginia, Fredericksburg, Va. Box 1005, College Station.

Burns, Sister M. Roberdette. Asso. Prof., Clarke Coll., Dubuque, Iowa.

Burns, R. F. Fribourg Univ., Fribourg, Switzerland. 1161 Eaton Ave., Beloit, Wis.

Burr, I. W. Asso. Prof., Purdue Univ., Lafayette, Ind.

Burroughs, Winifred K. Univ. of Michigan, Ann Arbor, Mich. 1001 E. Huron St. Burt, Mary M. (Mrs. L. N.). Retired. 800 24th Ave. N., St. Petersburg 4, Fla. Burton, L. J. Asst. Prof., Bryn Mawr Coll., Bryn Mawr, Pa. College Inn.

Burwell, Dr. W. R. Chm. of Board, Brush Development Co., 3311 Perkins Ave., Cleveland 14, Ohio.

Busemann, Herbert. Prof., Univ. of Southern California, Los Angeles 7, Calif.

Bush, K. A. 7 Walling Blvd., Oneonta, N.Y.

Bush, L. E. Prof., Coll. of St. Thomas, St. Paul 1, Minn.

Bushey, Jewell H. (Mrs. J. H.). Asso. Prof., Hunter Coll., New York 21, N.Y. Bushey, J. H. Asso. Prof., Hunter Coll., New York 21, N.Y.

Bushkovitch, A. V. Asso. Prof., Physics Dept., St. Louis Univ., St. Louis 3, Mo.

Busk, Th $\varnothing$ ger. Lect., Univ. of Copenhagen, Copenhagen $\varnothing$, Denmark. $41^{\text {III }}$ Tvaergade Dr., Copenhagen $\mathrm{K}$.

Bussey, W. H. Prof. Emeritus, Univ. of Minnesota, Minneapolis 14, Minn. 1421 East River Rd.

Butchart, J. H. Prof., Arizona State Coll., Flagstaff, Ariz.

Butler, J. B. Teaching Asst., Univ. of Virginia, Charlottesville, Va.

Butler, J. W. Junior Tech. Specialist, Argonne National Lab., P.O. Box 5207, Chicago 80, I11. 6146 Kenwood Ave., Chicago 37.

Butler, L. C. Instr., Alfred Univ., Alfred, N.Y. Box 998.

Butler, L. G. Instr., State Coll. of Washington, Pullman, Wash. 1904 B St. 
Butter, Dr. F. A., Jr. Math., Howard Hughes Aircraft Co., Culver City, Calif. 1030 Embury St., Pacific Palisades, Calif.

Byerly, R. W. 225 Broadway, New York 7, N.Y.

Byrd, P. F. Asst. Prof., Fisk Univ., Nashville 8, Tenn.

Byrne, Dr. Lee. Lect., Arizona State Coll., Tempe, Ariz. 6041 Dorchester Ave., Chicago 37, Ill.

Byrne, R. E. Asso. Prof, of Applied Mech., California Inst. of Tech., Pasadena 4, Calif. 335 S. Greenwood Ave., Pasadena 10.

Byrne, W. E. Prof., Virginia Military Inst., Lexington, Va. Box 836.

Cahn, A. S. Executive Officer, Inst. for Numerical Analysis, National Bur. of Standards, Univ. of California at Los Angeles, Los Angeles 24, Calif.

Cairns, S. S. Prof., Univ. of Illinois, Urbana, Ill.

Cairns, W. D. Prof. Emeritus, Oberlin Coll., Oberlin, Ohio; Lect., California Inst. of Tech., Pasadena 4, Calif.

Caldwell, G. O. Prof., Rust Coll., Holly Springs, Miss. Box 645.

Calkin, J. W. Asso. Prof., Rice Inst., Houston 1, Tex.

Calkins, Helen. Prof., Pennsylvania Coll. for Women, Pittsburgh 6, Pa.

Callahan, Ethel B. Prof., Hartwick Coll., Oneonta, N.Y. 122 Chestnut St.

Callahan, W. R. Asst. Prof., New York Univ., New York 53, N.Y. 121 Gould Hall.

Calloway, Sister Rose G. Instr., Mt. St. Mary's Coll., Los Angeles 24, Calif. 12001 Chalon Rd.

Cameron, E. A. Prof., Univ. of North Carolina, Chapel Hill, N.C. 121 Kenan St.

Cameron, R. H. Prof., Univ. of Minnesota, Minneapolis 14, Minn. 4401 15th Ave. S., Minneapolis 7.

†Camp, B. H. Prof. Emeritus, Administrator, and Contract Officer for Government Research, Wesleyan Univ., Middletown, Conn. 110 Mt. Vernon St.

Camp, C. C. Prof., Univ. of Nebraska, Lincoln 8, Neb.

Camp, E. J. Prof., Macalester Coll., St. Paul 5, Minn.

Campaigne, Dr. H. H. Math., Communications Station, Navy Dept., Washington 16, D.C. 210 N. Granada St., Arlington, Va.

Campbell, Dr. D. F. Consulting Actuary, 188 W. Randolph St., Chicago 1, I11.

Campbell, Frances L. Prof., George Pepperdine Coll., Los Angeles 44, Calif.

Campbell, Dr. G. A. Retired. 129 Bellevue Ave., Upper Montclair, N.J.

Campbell, G. C. Asst. Actuary, Metropolitan Life Ins. Co., 1 Madison Ave., New York 10, N.Y.

Campbell, H. E. Asst., Univ. of Wisconsin, Madison 6, Wis. 705 Langdon St., Madison 5 .

Campbell, J. D. Ässo. Prof., Rensselaer Polytech. Inst., Troy, N.Y.

Campbell, J. w. Prof., Univ. of Alberta, Edmonton, Alta., Canada.

Campbell, R. C. Asst. Prof., U. S. Naval Postgrad. School, Annapolis, Md. 120 Charles St.

Campbell, Willie B. Instr., Southern Univ., Baton Rouge, La. 1618 Pennsylvania Ave., Austin 22, Tex.

†Canning, Joseph. Roundhouse Foreman, Northern Pacific Railway Co., Garrison, Mont.

Cannon, Dr. E.W. Asst. Chief, Applied Math. Div., National Bur. of Standards, Commerce Dept., Washington 25, D.C. 5 Vassar Circle, Glen Echo, Md.

Capecelatro, Achille. Asst. Prof., Physics Dept., Newark Coll. of Eng., Newark 2, N.J. Box 91, Mt. Arlington, N.J.

Capo, Dr. B. G. Asst. Dir. for Research, Agric. Experimental Station, Rio Piedras, Puerto Rico. 102 Arzuaga St.

Caraher, Loula M. Asso. Prof., Arkansas State Teachers Coll., Conway, Ark. 343 Donaghey Ave.

Carey, C. E. Asso. Prof., Greenville Coll., Greenville, I11. 223 Asbury Ave.

Carey, E. F. A. Asso. Prof. Emeritus, Montana State Univ., Missoula, Mont.

Caris, P. A. Asso. Prof., Univ. of Pennsylvania, Philadelphia 4, Pa. 717 Shadeland Ave., Drexel Hill, Pa.

Carlen, Mildred E. Registrar, Grad. School, Brown Univ., Providence 12, R.I. 
Carlitz, Leonard. Prof., Duke Univ., Durham, N.C. Box 4674, Duke Station. Carlson, B. G. Los Alamos Scientific Lab., Univ. of California, P.O. Box 1663, Los Alamos, N.M.

Carlson, C. S. Prof., St. Olaf Coll., Northfield, Minn.

Carlson, Elizabeth. Asst. Prof., Univ. of Minnesota, Minneapolis 14, Minn. 302414 th Ave. S., Minneapolis 7.

Carlson, K. H. Asst. Prof., Valparaiso Univ., Valparaiso, Ind.

Carlson, P. G. Instr., Rensselaer Polytech. Inst., Troy, N.Y. Carnegie Bldg.

Carlton, L. Virginia. Asso. Prof., Northwestern State Coll., Natchitoches, La.

Carman, M. G. Prof., Murray State Teachers Coll., Murray, Ky.

Carmichael, R. D. Dean Emeritus and Prof. Emeritus, Univ, of Illinois, Urbana, I1l. Griggsville, I11.

Carner, F. E. Design Eng., Eng. Dept., Aviation Gas Turbine Div., Westinghouse Elec. Corp., Essington, Pa. 1404 N. Park Ave., Philadelphia 22, Pa.

Carpenter, A. F. Prof., Univ. of Washington, Seattle 5, Wash. Physics Hall.

Carpenter, Fay D. Vibration Eng., Glenn L. Martin Co., Baltimore, Md. 79 Dunkirk Rd., Baltimore 12.

Carpenter, F. M. Asst. Prof., Colorado School of Mines, Golden, Colo.

Carr, R. E. Asst. Prof., Michigan State Coll., East Lansing, Mich.

Carrier, G. F. Asso. Prof., Grad. Div. of Applied Math., Brown Univ., Providence 12, R.I. 27 Brown St., Providence 6.

Carroll, I. S. Prof., Syracuse Univ., Syracuse 10, N.Y. 511 Comstock Ave.

Carroll-Rusk, Evelyn (Mrs. W. S. Rusk). Dean and Prof., Wells Coll., Aurora, N.Y.

Carruth, P. W. Asst. Prof., Swarthmore Coll., Swarthmore, Pa. 317 N. Chester $\mathrm{Rd}$.

Carslaw, H. S. Prof. Emeritus, Univ. of Sydney, Sydney, New South Wales, Australia. Burradoo, New South Wales, Australia.

Carson, A. B. Asso. Prof., Air Forces Inst. of Tech., Wright Field, Ohio. 301 Dawnview Ave., Dayton 3, Ohio.

Carson, E. R. I. Instr., St. Francis Coll., Brooklyn 2, N.Y. 142 Henry St.

Carter, E. R. Actuary, Washington National Ins. Co., Evanston, Ill.

Carter, H. C. Prof., Mary Washington Coll., Univ. of Virginia, Fredericksburg, Va. 1307 Littlepage St.

Carter, P. S. Radio Research Eng., Labs. Div., Radio Corp. of America, Rocky Point, N.Y.

Carter, Dr. W. C. Math., Air Flow Branch, Exterior Ballistics, Ballistic Research Labs., Aberdeen Proving Ground, Md. 569 Fountain St., Havre de Grace, Md.

Cartwright, K. C. Eng. Designer, Columbia Chemical Div., Pittsburgh Plate Glass Co., Barberton, Ohio. 729 Wooster Rd. W.

de Carvalho, C. A. 41 Aripuana St. (bairro: Laranjeiras), D.F., Brazil.

Carver, W. B. Prof. Emeritus, Cornell Univ., Ithaca, N.Y. White Hall.

Case, J. E. Prof., St. Louis Univ., St. Louis 3, Mo.

Cashwell, E. D. Univ. of Wisconsin, Madison 6, Wis. North Hall.

Casse1, C. W. Instr., Univ. of Dayton, Dayton 9, Ohio. 861/2 Mary Ave., Dayton 5.

Cassen, P. N. Univ. of Michigan, Ann Arbor, Mich. 1552 Essex Ct., Willow Run, Mich.

Cassidy, Dr. W. F. St. John's Univ., Brooklyn 2, N.Y.

Cassity, Dr. C. R. Math., Research and Development Div., New Mexico School of Mines, Albuquerque, N.M. Box 6000, Station A.

Castle, S. N. Retired. 2425 University Ave., Honolulu 5, Hawaii.

Castrucci, Benedito. Prof., Univ. of São Paulo, São Paulo, Brazil. 111 Nicolau de Souza Queirós St.

Cates, G. T. Asst., Univ. of Illinois, Urbana, I11. 904 S. Anderson St.

Cathcart, Alan. Columbia Univ., New York 27, N.Y. 140 Pinehurst Ave., New York 33, N.Y.

Caton, W. B. Asst. Prof., State Coll. of Washington, Pullman, Wash.

Cattell, Alberta S. (Mrs. R. B.). 313 W. Avondale, Champaign, IIl. 
Catunda, Omar. Prof., Univ. of São Paulo, São Paulo, Brazil. 212 Alameda Santos.

Caywood, Dr. T. E. Senior Math., Ordnance Research, Univ. of Chicago, Chicago 37, I11. 5226 W. Congress St., Chicago 44.

Cederberg, W. E. Prof., Augustana Coll. and Theological Seminary, Rock Island, I11. $254222 \mathrm{x} / 2$ Ave.

Celauro, F. L. Prof., Newark Coll. of Eng., Newark 2, N.J.

Cell, J. W. Prof., State Coll. of Agric. and Eng., Univ. of North Carolina, Raleigh, N.C. Box 5548, State College Station.

Certaine, Jeremiah. Asst. Prof., Howard Univ., Washington 1, D.C. 45 E. 135th St., New York 35, N.Y.

Cesco, R. P. Prof., Univ. of LaPlata, LaPlata, Argentina. 389 66th St.

Chako, Nicholas. Asso. Prof., Physics Dept., Alabama Polytech. Inst., Auburn, Ala.

Chalfant, A. I. Analytical Eng., Pratt and Whitney Aircraft Corp., East Hartford, Conn. 548 Main St., Portland, Conn.

Chamberlin, R. E. Asst. Prof., Univ. of Utah, Salt Lake City 1, Utah.

Chand, Pratap. 2 Hardinge Ave., New Delhi, India.

Chandrasekhar, Subrahmanyan. Prof., Yerkes Observ., Univ. of Chicago, Williams Bay, Wis.

Chandrasekharan, Dr. K. Member, Inst. for Advanced Study, Princeton, N.J.

Chanler, Josephine H. Asst. Prof., Univ. of Illinois, Urbana, Ill. 364 Mathematics Bldg.

Chapline, J. D. Tech. Asst., Eckert-Mauchly Computer Corp., Broad and Spring Garden Sts., Philadelphia 23, Pa. 411 W. Mt. Airy Ave., Philadelphia 19.

Chapman, D. G. Asst., Stat. Lab., Univ. of California, Berkeley 4, Calif.

Charnes, Abraham. Asst. Prof., Carnegie Inst. of Tech., Pittsburgh 13, Pa.

Charney, Dr. J. G. Research Ässo., Electronic Computer Project, Inst. for Advanced Study, Princeton, N.J.

Charpentier, Marie R. J. Prof., Univ. of Rennes, Rennes, France.

Chatelet, Albert. Prof., Univ. of Paris, Paris, France. 120 Assas St., Paris 6, France.

Chatland, Harold. Asso. Prof., Graduate Center, Ohio State Univ., Wright Field, Ohio. 4377 Olentangy Blvd., Columbus 2, Ohio.

Chelius, L. G. Physicist, Carbide and Carbon Chemicals Corp., Oak Ridge, Tenn. 105 Euclid P1.

Chellevold, J. O. Asst. Prof., Lehigh Univ., Bethlehem, Pa. 1111 Maple St.

Chen, K. K. Prof., Chekiang Univ., Hangchow, Chekiang, China.

Chen, K. T. Columbia Univ., New York 27, N.Y.'John Jay Hall.

Chen, P. P. Brown Univ., Providence 12, R.I. 121 Power St., Providence 6.

Chen, Dr. W. M. 814 Mandana Blvd., Oakland 10, Calif.

Chen, Dr. Y. W. Research Asso., Inst. for Math. and Mech., New York Univ., New York 3, N.Y. 150-46 Village Rd., Parkway Village, Jamaica 2, N.Y.

Cheney, W. F. Prof., Univ. of Connecticut, Storrs, Conn. Box 26.

Chern, S. S. Prof., Inst. of Math., Chinese Acad., 1 Jeou Hwa Shan, Nanking 5, China.

Chernick, Jack. Asso. Scientist, Brookhaven National Lab., Upton, N.Y.

Chernoff, Herman. Cowles Comm. for Research in Economics, Univ. of Chicago, Chicago 37, I11.

Chernofsky, M. I. Lect., City Coll., New York 10, N.Y. P.O. Box 81, St. John's Place Station, Brooklyn 13, N.Y.

Cherry, T. M. Prof., Univ. of Melbourne, Melbourne N. 3, Victoria, Australia.

Chesna, John. Electro-optical Physicist, Camera Works, Eastman Kodak Co., Rochester 4, N.Y. 12 Otilla St., Rochester 5.

Chessin, P. L. Columbia Univ., New York 27, N.Y. 316 W. 93d St., New York 25.

Chevalley, Claude. Prof., Princeton Univ., Princeton, N.J. Fine Hall.

Cheydleur, B. F. Math., Naval Ordnance Lab., Naval Gun Factory, Washington 25, D.C. 602 Ave. E, Washington 19.

Chiang, L. F. Prof., Inst. of Math., Chinese Acad., 1 Jeou Hwa Shan, Nanking 5, China. 
Chiarulli, Peter. Asso., Grad. Div. of Applied Math., Brown Univ., Providence 12, R.I.

Chiefa, S. R. 141 Jasper St., Paterson 2, N.J.

Chien, W. Z. Prof., Mech. Eng. Dept., Tsing Hua Univ., Peiping, China.

Chin, Louise H. Asst. Prof., Univ. of Arizona, Tucson, Ariz.

Chittenden, E. W. Prof., State Univ. of Iowa, Iowa City, Iowa. 1101 Kirkwood Ave.

Choquet, Dr. Gustave. Lect., Univ. of Grenoble, Grenoble, France. Institut Fourier.

Chow, W. L. Ásso. Prof., Johns Hopkins Univ., Baltimore 18, Md.

Chowla, Dr. Sarvadaman. Member, Inst. for Advanced Study, Princeton, N.J.

Christiansen, Alice M. Instr., Wright Junior Coll., Chicago 34, I11. $2149 \mathrm{~N}$. Sayre Ave., Chicago 37.

Christie, D. E. Asst. Prof., Bowdoin Coll., Brunswick, Me. 36 Boody St.

Chung, Dr. K. L. Asst. Prof., Cornell University, Ithaca, N.Y. White Hall.

Church, Alonzo. Prof., Princeton Univ., Princeton, N.J. Fine Hall.

Church, Elsie T. Instr., Univ. of Kentucky, Lexington 29, Ky.

Church, Randolph. Prof., U.S. Naval Postgrad. School, Annapolis, Md. 316 N. Glen Ave.

Churchill, Edmund. Asst. Prof., Antioch Coll., Yellow Springs, Ohio.

Churchill, R. V. Prof., Univ. of Michigan, Ann Arbor, Mich. 924 S. Forest Ave.

Civin, Paul. Asst. Prof., Univ. of Oregon, Eugene, Ore.

Clamons, E. H. Instr., Mech. Eng. Dept., Univ. of Minnesota, Minneapolis 14, Minn. 1696 Beechwood Ave., St. Paul 5, Minn.

Clark, C. E. Asso. Prof., Emory Univ., Emory University, Ga.

Clark, C. J. Research Eng., Curtiss-Wright Aircraft Corp., Columbus, Ohio. 2513 Avalon P1.

Clark, C. L. Asso. Prof., Oregon State Coll., Corvallis, Ore.

Clark, C. R. Teacher, McKinley High School, Washington, D.C. 2707 Adams Mill Rd. N.W., Washington 9.

Clark, Dr. F. E. Instr., Tulane Univ. of Louisiana, New Orleans 15, La.

Clark, Helen M. Instr., Northwestern Univ., Evanston, III.

Clark, Joan R. (Mrs. C. R.). Development Eng., Brown Instrument Co., Wayne and Roberts Aves., Philadelphia 44, $\mathrm{Pa}$.

Clark, W. G. Asst. Prof., Mt. Union Coll., Alliance, Ohio.

Clarke, A. B. Asst., Brown Univ., Providence 12, R.I.

Clarke, E. H. Prof., Hiram Coll., Hiram, Ohio. Box 283.

Clarke, F. Marion. Instr., Univ. of Nebraska, Lincoln 8, Neb.

Clarkson, Helen E. Instr., Creighton Univ., Omaha 2, Neb. 528 S. 52d St., Omaha 6.

Clarkson, J. A. Asso. Prof., Univ. of Pennsylvania, Philadelphia 4, Pa. Box 13, College Hall.

Clarkson, J. M. Prof., State Coll. of Agric. and Eng., Univ. of North Carolina, Raleigh, N.C. State College Station.

Clarkson, M. H. Research Eng., Defense Research Lab., Univ. of Texas, Austin 12, Tex.

Claytor, W. W. S. Asso. Prof., Howard Univ., Washington 1, D.C.

Clement, Mary Dean. Asst. Prof., Univ. of Miami, Coral Gables 34, Fla.

Clement, P. A. Teaching Asst., Univ. of California at Los Angeles, Los Angeles 24, Calif.

Clements, G. R. Prof., U.S. Naval Acad., Annapolis, Md. 7 Thompson St.

Clifford, A. H. Asso. Prof., Johns Hopkins Univ., Baltimore 18, Md.

Cline, Lois $\mathbf{V}$. Asst., Univ. of Illinois, Urbana, Ill. 1106 W. California.

Clippinger, Dr. R. F. Ballistician, Army Ordnance Dept., Aberdeen Proving Ground, Md. 102 O'Neill Ct., Havre de Grace, Md.

Coble, A. B. Visiting Prof., Haverford Coll., Haverford, Pa.

Coburn, Nathaniel. Asst. Prof., Univ. of Michigan, Ann Arbor, Mich. 1304 Washtenaw Ter.

Cockshott, A. H. Instr., Manhattan Coll., New York 63, N.Y. Box 308.

Cocuzza, P. J. City Coll., New York 31, N.Y. 2571 W. 16th St., Brooklyn 14, N.Y.

Coddington, Dr. E. A. Instr., Johns Hopkins Univ., Baltimore 18, Md. 2934 St. Paul St. 
Coe, C. J. Asst. Prof., Univ. of Michigan, Ann Arbor, Mich. 3004 Angell Hall. Coffin, L. M. Prof. Emeritus, Coe Coll., Cedar Rapids, Iowa. 633 S. Bixel St., Los Angeles 14, Calif.

Cohen, Abraham. Prof. Emeritus, Johns Hopkins Univ., Baltimore 18, Md.

Cohen, A. C. Asso. Prof., Univ. of Georgia, Athens, Ga. 399 W. Southview Dr.

Cohen, Carl. Research Asso., Harvard Univ., Cambridge 38, Mass. 40 Prentiss St., Cambridge 40.

Cohen, Dr. Eckford. Instr., Syracuse Univ., Syracuse 10, N.Y.

Cohen, Haskell. Instr., Univ. of Alabama, University, Ala. Box 2922.

Cohen, H. J. Asst., Univ. of Wisconsin, Madison 6, Wis. North Hall.

Cohen, I. S. Asst. Prof., Massachusetts Inst. of Tech., Cambridge 39, Mass.

Cohen, L. W. Asst. Prof., Queens Coll., Flushing, N.Y. 217-18 48th Ave., Bayside, N.Y.

Cohen, Teresa. Prof., Pennsylvania State Coll., State College, Pa. 315 S. Atherton St.

Cohn, Harvey. Asst. Prof., Wayne Univ., Detroit 1, Mich.

Cohn, Dr. R. M. Instr., Rutgers Univ., New Brunswick, N.J. 905 West End Ave., New York 25, N.Y.

Coker, E. C. Asso. Prof., Clemson Coll., Clemson, S.C. Box 1121.

Cole, C. A. 2d Lt., U.S.M.C., Marine Air Control Group 1, Marine Corps Air Station, Cherry Point, N.C.

Cole, K. S. Prof., Inst. of Radiobiology and Biophysics, Univ. of Chicago, Chicago 37, I11.

Cole, Nancy. Asst. Prof., Syracuse Univ., Syracuse 10, N.Y.

Cole, R. H. Asso. Prof., Univ. of Western Ontario, London, Ont., Canada. Asst. Prof., Princeton Univ., Princeton, N.J.

Coleman, Dr. A. J. Sec., World's Student Christian Federation, 13 Calvin St., Geneva, Switzerland.

Coleman, J. B. 620 Bull St., Columbia, S.C.

Coleman, W. B. Univ. of Texas, Austin 12, Tex. 4404 Shoalwood Ave., Austin 21.

Coler, M. A. Asst. Prof., Coll. of Eng., New York Univ., New York 53, N.Y. 785 West End Ave., New York 25.

Collier, Myrtie. Prof. Emeritus, Immaculate Heart Coll., Los Angeles 27, Calif. $225 \mathrm{~S}$. Thurston Ave., Los Angeles 24.

Collingwood, Dr. E. F. Lilburn Tower, Alnwick, Northumberland, England.

†Collins, O. C. Asst. Prof., Astronomy Dept., Univ. of Nebraska, Lincoln 8, Neb. The Observatory.

Colon, J. A. Instr., Univ. of Puerto Rico, Rio Piedras, Puerto Rico. Inst. of Tropical Meteorology.

Colquitt, L. A. Asst. Prof., Texas Christian Univ., Ft. Worth, Tex.

Colson, H. D. Teacher, Bemidji State Teachers Coll., Bemidji, Minn.

Colvin, B. H. Asst. Prof., Univ. of Wisconsin, Madison 6, Wis. North Hall.

Comba, Paolo. Asst., California Inst. of Tech., Pasadena 4, Calif.

Comegys, Esther. Asst. Prof., Univ. of Maine, Örono, Me. Dryden Ter., 17 Harris Rd.

Comenetz, Dr. George. Research Eng., Westinghouse Research Labs., Westinghouse Elec. Corp., East Pittsburgh 30, Pa.

Comfort, E. G. H. Asst. Prof., Illinois Inst. of Tech., Chicago 16, I11. 812 Prairie Ave., Wilmette, Ill.

Comstock, C. E. Prof. Emeritus, Bradley Univ., Peoria 5, I11. 203 Fredonia Ave.

Conkwright, N. B. Asso. Prof., State Univ, of Iowa, Iowa City, Iowa. 209-B Physics Bldg.

Conlan, Sister M. Loyola. Asso. Prof., Coll. of Mt. St. Vincent, New York 63, N.Y.

Connelly, Brother Damian. Asst. Prof., La Salle Coll., Philadelphia 41, Pa.

Conrad, W. A. Prof., U.S. Naval Acad., Annapolis, Md.

Constable, Mary Louise. Teacher, Philadelphia High School for Girls, Philadelphia 30, Pa. The Whittier, 140 N. 15th St., Philadelphia 2.

Conwell, Dr. G. M. 264 Dearing St., Athens, Ga. 
Conwell, H. H. Dean and Prof., Beloit Coll., Beloit, Wis. 1621 Emerson St. Cook, A. J. Asso. Prof., Univ. of Alberta, Edmonton, Alta., Canada.

Cook, Erben. Asst. Prof., Univ. of Connecticut, Storrs, Conn. Box 48.

Cook, E. A. Research Physicist, Research Lab., Carter Oil Co., 1133 N. Lewis, Tulsa, Okla.

Cook, Sister Rose M. Prof., Loretto Heights Coll., Loretto, Colo.

Cooley, H. R. Prof., New York Univ., New York 3, N.Y.

Cooley, J. A. Prof., Univ. of Tennessee, Knoxville 16, Tenn.

†Coolidge, J. L. Prof. Emeritus, Harvard Univ., Cambridge 38, Mass. 27 Fayerweather St.

Coolidge, T. J. Vice Pres., First National Bank of Boston, 67 Milk St., Boston 6, Mass.

Cooper, Dr. Elizabeth M. Teacher, Hunter Coll. High School, New York 21, N.Y. 201 E. 71st St.

Cope, T. F. Prof., Queens Coll., Flushing, N.Y. Montrose, N.Y.

Copeland, A. H. Prof., Univ. of Michigan, Ann Arbor, Mich. 616 Oswego St.

†Copeland, Lennie P. Prof. Emeritus, Wellesley Coll., Wellesley 81, Mass. 1190 8th St. N., St. Petersburg, Fla.

Coplan, Natalie. Computer, Computation Lab., National Bur. of Standards, 150 Nassau St., New York 7, N.Y. 365 West End Ave., New York 24.

Copson, E. T. Prof., University Coll., Univ. of St. Andrews, Dundee, Scotland.

Coral, Max. Asso. Prof., Wayne Univ., Detroit 1, Mich.

Corliss, J. J. Asso. Prof., Univ. of Illinois, Chicago 11, I11.

van der Corput, J. G. Prof., Univ. of Amsterdam, Amsterdam, Netherlands. 5 Wyttenbachstraat, Amsterdam O.

Cortell, Robert. Asst. Prof., City Coll., New York 31, N.Y.

Cosby, Byron. Asst. Prof., State Univ. of Iowa, Iowa City, Iowa. 209-A Physics Bldg.

Costello, G. R. Math., Flight Propulsion Research Lab., National Advisory Com. for Aeronautics, Cleveland 11, Ohio. 219 Seminary St., Berea, Ohio.

Cotellessa, R. F. Columbia Univ., New York 27, N.Y. Box 162, Ridgewood, N.J.

Cothran, F. E. 709 S. Norton Ave., Los Angeles 5, Calif.

Cothran, J. C. Prof., Chemistry Dept., Univ. of Minnesota, Duluth 5, Minn. 512 N. 19th Ave. E.

Cotlar, Micha. Research Math., Univ. of Buenos Aires, Buenos Aires, Argentina. 1309 Sarandi.

Courant, Richard. Prof., Inst. for Math. and Mech., New York Univ., New York 3, N.Y.

Court, L. M. Asst. Prof., Rutgers Univ., New Brunswick, N.J.

Court, N. A. Prof., Univ. of Oklahoma, Norman, Okla. 425 W. Eufaula St.

Coveyou, R. R. Asso. Physicist, Oak Ridge National Lab., Oak Ridge, Tenn.

Cowen, Elaine V. (Mrs. Jerry). Instr., Wellesley Coll., Wellesley 81, Mass. 85 N. Central St., East Bridgewater, Mass.

Cowgill, Dr. A. P. Maj. U.S.A., retired. 519 Allen St., Syracuse 10, N.Y.

Cowles, W. H. H. Prof., Pratt Inst., Brooklyn 5, N.Y. 132 Joralemon St., Brooklyn 2.

Cowling, T. G. Prof., Univ. of Leeds, Leeds 2, England. 19 Hollin Gardens, Leeds 6.

Cowling, V. F. Asst. Prof., Lehigh Univ., Bethlehem, Pa.

Cox, E. F. Prof., Howard Univ., Washington 1, D.C.

Cox, Dr. L. C. English Hotel, Indianapolis 4, Ind.

Cox, Mary J. Math., Ballistic Research Labs., Aberdeen Proving Ground, Md. Box 416, Aberdeen, Md.

Cox, R. F. Dean, John Brown Univ., Siloam Springs, Ark.

Coxeter, H. S. M. Prof., Univ. of Toronto, Toronto 5, Ont., Canada. 24 Strathearn Blvd., Toronto 10.

Coyne, Dorothy I. Instr., Paul Smith's Coll. of Arts and Sciences, Paul Smiths, N.Y.

Crabtree, J. B. Instr., Univ. of Chicago, Chicago 37, I11.

Craig, A. T. Prof., State Univ. of Iowa, Iowa City, Iowa. 208 Physics Bldg. 
Craig, C. C. Dir., Stat. Research Lab., and Prof., Univ. of Michigan, Ann Arbor, Mich. 3020 Angell Hall.

Craig, H. V. Prof., Univ. of Texas, Austin 12, Tex. 3104 Grandview St., Austin.

Cramblet, W. H. Pres., Bethany Coll., Bethany, W.Va.

Cramer, Dr. G. F. Math., Navy Dept., Washington 25, D.C. 112 Quincy St., Chevy Chase $15, \mathrm{Md}$.

Cramér, Harald. Prof., Univ. of Stockholm, Stockholm, Sweden. 7 Skärviksvägen, Djursholm, Sweden.

Cramer, R. H. Aerodynamicist, Cornell Aeronautical Lab., Inc., 4455 Genesee St., Buffalo, N.Y. 500 Grand Ave., Rochester 9, N.Y.

Cramlet, C. M. Prof., Univ. of Washington, Seattle 5, Wash. 11060 Sand Point Way, Seattle 55.

Crandall, S. H. Asst. Prof., Mech. Eng. Dept., Massachusetts Inst. of Tech., Cambridge 39, Mass.

Crane, C. H. Pres., St. Joseph Lead Co., 250 Park Ave., New York 17, N.Y.

Craver, F. E. Retired. 262 Gibson Ct., Carlisle, Pa.

Craw, A. R. Asst. Prof., U.S. Naval Acad., Annapolis, Md.

Crawford, Jane E. Eng., General Elec. Co., Schenectady 5, N.Y. 1010 Regent St., Schenectady 8.

Crawford, w. S. H. Prof., Mt. Allison Univ., Sackville, N.B., Canada. Box 174.

Crawley, A. B. West Virginia State Coll., Institute, W.Va., Box 174.

Creagh, Kathleen. Teacher, Waller Branch High School, Chicago 25, I11. 4526 N. Whipple St.

Creane, Sister James S. Asst. Prof., Fontbonne Coll., St. Louis 5, Mo.

Creese, F. G. San Francisco State Coll., San Francisco 2, Calif. 269 Davis St., San Leandro, Calif.

Crisler, E. H. Instr., West Virginia Univ., Morgantown, W.Va. 138 King St.

Crispin, J. W. Instr., Wayne Univ., Detroit 1, Mich. 363 Richton Ave., Detroit 3.

Crist, F. M. Col. U.S.A., retired. 8305 Childs Rd., Philadelphia 18, Pa.

Critchlow, Florence G. (Mrs. G. F.). Tutor, Queens Coll., Flushing, N.Y. 42-75 Kissena Blvd.

Cromwell, J. W. Certified Public Accountant, 1815 13th St. N.W., Washington 9, D.C.

Cronin, Dr. Jane S. Fellow, Princeton Univ., Princeton, N.J. 28 Vanderventer Ave.

Cronvich, Dr. L. L. Math., Applied Physics Lab., Johns Hopkins Univ., Silver Spring, Md. 8712 Colesville Rd.

Crosby, Dr. D. R. Fellow, Princeton Univ., Princeton, N.J. Fine Hall.

Crosby, W. J. R. Asst. Prof., Univ. of Saskatchewan, Saskatoon, Sask., Canada. 908 Saskatchewan Crescent E.

Crouch, R. P. Teaching Asst., Texas Tech. Coll., Lubbock, Tex. 2418 7th St.

Crouse, C. W. Actuary, Manufacturers Casualty Ins. Co., 1617 Pennsylvania Blvd., Philadelphia 3, $\mathrm{Pa}$.

Crout, P. D. Asso. Prof., Massachusetts Inst. of Tech., Cambridge 39, Mass. 2-173.

Crow, Dr. E. L. Math., Research Dept., Naval Ordnance Test Station, China Lake, Calif. 105-B Ellis St.

Culbertson, J. T. Fellow, Math. Biophysics, Univ. of Chicago, Chicago 37, I11.

Culmer, W. J. A. Asst. Prof., Hamline Univ., St. Paul 4 E, Minn.

Culver, M. M. Prof., Univ. of Pitsburgh, Pittsburgh 13, Pa.

Cummings, H. K. Chief, Automotive Power Plants Sec., National Bur. of Standards, Commerce Dept., Washington 25, D.C. 4213 Ellicott St. N.W., Washington 16.

Cunningham, A. B. Asst. Prof., West Virginia Univ., Morgantown, W.Va.

Cunningham, Frederic. Teaching Fellow, Harvard Univ., Cambridge 38, Mass. $35 \mathrm{Ash}$ St.

Cunningham, L. E. Asso. Prof., Univ. of California, Berkeley 4, Calif. Students' Observatory.

Currie, J. C. Asso. Prof., Alabama Polytech. Inst., Auburn, Ala.

Curry, H. B. Prof., Pennsylvania State Coll., State College, Pa.

Curtis, H. B. Prof., Lake Forest Coll., Lake Forest, Ill. 11 College Campus. 
Curtis, H. J. Instr., Illinois Inst. of Tech., Chicago 16, I1l. 934 Bonnie Brae, River Forest, Ill.

Curtiss, D. R. Prof. Emeritus, Northwestern Univ., Evanston, I1l. 1249 Monterey Ave., Redlands, Calif.

Curtiss, Dr. J. H. Chief, National Applied Math. Labs., National Bur. of Standards, Commerce Dept. Washington 25, D.C.

Cutler, E. H. Asso. Prof., Lehigh Univ., Bethlehem, Pa.

Dadourian, H. M. Prof., Trinity Coll., Hartford 6, Conn. 125 Vernon St.

$\lceil$ Dalal, R. D. Retired. Care of Swiss Bank Corp., 11c Regent St., Waterloo P1., London S.W. 1, England.

Daly, Dr. J. F. Stat., Bur. of the Census, Commerce Dept., Washington 25, D.C. 421318 th St. N.E., Washington 18

Dana, Victor. Fouad I Univ., Cairo, Egypt. Care of Sirgy, Dana and Co., 9 Adley Pacha St.

Daniells, Marian E. Asst. Prof., Iowa State Coll. of Agric. and Mech. Arts, Ames, Iowa.

Danskin, J. M. Fellow, Univ. of California, Berkeley 4, Calif. International House.

Dantzig, Dr. G. B. Math. Advisor to Comptroller, Air Forces, Washington 25, D.C. 3203 N. 19th Rd., Arlington, Va.

Dantzig, Dr. Tobias. Retired. 2321 Lake View, Los Angeles 26, Calif.

D’Arco, Paul. Instr., DePaul Univ., Chicago 1, I11. 1116 W. Polk St., Chicago 7.

Darkow, Marguerite D. Asso. Prof., Hunter Coll., New York 21, N.Y. 16 E. 82d St., New York 28.

Darling, D. A. Asst. Prof., Rutgers Univ., New Brunswick, N.J.

Daum, J. A. Asst. Prof., Agric. and Mech. Coll. of Texas, College Station, Tex. Box 1011.

Daus, P. H. Prof., Univ. of California at Los Angeles, Los Angeles 24, Calif.

Davenport, Harold. Prof., University Coll., Univ. of London, London W.C.1, England.

Davids, Norman. Asso. Prof., Pennsylvania State Coll., State College, Pa. Ordnance Research Lab.

Davidson, Anthony. Aeronautical Eng., Air Materiel Command, Wright Field, Ohio. 455 Groveland Ave., Dayton 7, Ohio.

Davidson, Nancy L. Math., Naval Ordnance Test Station, China Lake, Calif. 210 B Fowler.

Davies, Robert. Univ. of Wisconsin, Madison 6, Wis. 1615 Hoyt St., Madison 5.

Davies, R. W. Univ. of Edinburgh, Edinburgh 1, Scotland.

Davis, Anne C. (Mrs. E. A.). Univ. of California, Berkeley 4, Calif. 2518 Ellsworth St.

Davis, D. R. Prof., New Jersey State Teachers Coll., Upper Montclair, N.J. 43 College Ave.

Davis, E. A. Asst. Prof., Univ. of Nevada, Reno, Nev.

Davis, H. A. Prof., West Virginia Univ., Morgantown, W.Va. 307 Duquesne Ave.

Davis, H. C. Harvard Univ., Cambridge 38, Mass. 44 Irving St.

$\dagger$ Davis, H. N. Pres., Stevens Inst. of Tech., Hoboken, N.J.

Davis, James Edgar. Asst. Prof., Coll. of Pharmacy, Univ. of Illinois, Chicago 12 , I11.

Davis, James Elmer. Prof., Drexel Inst. of Tech., Philadelphia 4, Pa.

Davis, Martin. Research Asst., Princeton Univ., Princeton, N.J. Fine Hall.

Davis, M. E. Actuary, Metropolitan Life Ins. Co., 1 Madison Ave., New York 10, N.Y.

Davis, Margaret R. Prof., Southeastern Louisiana Coll., Hammond, La.

Davis, Philip. Teaching Fellow, Harvard Univ., Cambridge 38, Mass. 2 Potter $\mathrm{Pk}$.

Davis, W. C. Eng., Heating and Air Conditioning Div., Debardeleben Coal Corp., 421 S. 21 st St., Birmingham 3, Ala.

Davis, W. M. Prof., Cornell Coll., Mt. Vernon, Iowa. 616 7th Ave. N. 
Davison, Charlotte I. Prof. Emeritus, Wilson Coll., Chambersburg, Pa. 1046 Edgar Ave.

Dawkins, W. S. Prof., Loyola Coll., Baltimore 10, Md. 4401 Bedford P1., Baltimore 18.

Dawson, Lester. Asso. Prof., Adams State Coll., Alamosa, Colo.

Dawson, R. E. Acting Dean and Instr., Jacksonville Junior Coll., Jacksonville 4, Fla. 4728 Pinewood Rd., Jacksonville 5.

Day, A. S. Asst. Prof., Wesleyan Univ., Middletown, Conn. Box 11, Wesleyan Station.

Day, M. M. Asso. Prof., Univ. of Illinois, Urbana, Ill. Member, Inst. for Advanced Study, Princeton, N.J.

Dean, Alice C. Lib. Emeritus, Rice Inst., Houston 1, Tex. P.O. Box 1892.

Dean, B. V. Instr., Columbia Univ., New York 27, N.Y. 35 W. 102d St., Orangeburg, N.Y.

Dearborn, D. C. Dean and Prof., Catawba Coll., Salisbury, N.C.

DeBaggis, Rev. H. F. Instr., Univ. of Notre Dame, Notre Dame, Ind. The Presbytery.

Debever, Dr. R. G. Lect., Free Univ. of Brussels, Brussels, Belgium. 46 Helmet St.

DeCesare, E. A. Prof., Military School of Rio Santiago. 1757 Rivadavia St., Buenos Aires, Argentina.

DeCew, w. M. Editor, Nucleonics, McGraw-Hill Publishing Co., 330 W. 42d St., New York 18, N.Y. 290 Claremont Ave., Mt. Vernon, N.Y.

Decherd, Mary E. Retired. 2313 Nueces St., Austin, Tex.

DeCicco, John. Asso. Prof., Illinois Inst. of Tech., Chicago 16, Ill. 6340 Blackstone Ave. Chicago 37.

Deck, L. J. Prof., Muhlenberg Coll., Allentown, Pa.

Decker, F. F. Prof., Syracuse Univ., Syracuse 10, N.Y. 312 Marshall St.

DeCleene, Rev. L. A. V. Prof., St. Norbert Coll., West DePere, Wis. 1015 S. Monroe Ave., Green Bay, Wis.

$\nmid$ Dederick, Dr. L. S. Asso. Dir., Ballistic Research Labs., Aberdeen Proving Ground, Md.

DeFoe, O. K. Prof., St. Louis Coll. of Pharmacy and Allied Sciences, St. Louis 10, Mo. 4588 Parkview P1.

Dehn, M. W. Prof., Black Mountain Coll., Black Mountain, N.C.

Deime1, R. F. Prof., Mech. Eng. Dept., Stevens Inst. of Tech., Hoboken, N.J.

Dekker, Dr. D. B. Instr., Univ. of Washington, Seattle 5, Wash.

Delange, Hubert. Prof., Univ. of Clermont-Ferrand, Clermont-Ferrand, France.

DelFranco, Georgia K. (Mrs. J.) Asst. Prof., Univ. of Miami, Coral Gables 34, Fla. 6126-8 S. Woodlawn Ave., Chicago 37, Ill.

Delsarte, J. F. Dean, Univ. of Nancy, Nancy, France.

DeLury, Dr. D. B. Dir., Math Stat. Dept., Ontario Research Foundation, 43 Queen's Park, Toronto 5, Ont., Canada.

Demers, M. R. Brown Univ., Providence 12, R.I. Instr., Univ. of Nevada, Reno, Nev.

Demir, Huseyin. E.K.I. Kaudilli Bolg., Eregli, Zonguldak, Turkey.

Denbow, C. H. Asso. Prof., U.S. Naval Postgrad. School, Annapolis, Md.

Denby-Wilks, J. E. Teaching Fellow, California Inst. of Tech., Pasadena 4, Calif. Norman Bridge Lab. of Physics.

Deniston, R. F. Instr., Iowa State Coll. of Agric. and Mech. Arts, Ames, Iowa. West of Wilmoth Rd.

Dennis, J. J. Prof., Clark Coll., Atlanta, Ga. 134 Ashby St. S.W.

Denton, W. W. Asst. Prof., Univ. of Arizona, Tucson, Ariz. 643 N. 4th Ave.

De Prima, C. R. Asst. Prof. of Applied Mech., California Inst. of Tech., Pasadena 4, Calif.

Derry, Douglas. Asso. Prof., Univ. of British Columbia, Vancouver, B.C., Canada. 4593 W. 5th Ave.

Deutsch, R. A. Elec. Eng., Kellex Corp., 233 Broadway, New York 7, N.Y. 4672 Broadway, New York 34.

DeWitt, Dorothy M. Instr., Iowa State Teachers Coll., Cedar Falls, Iowa.

Diamond, A. H. Prof., Oklahoma Agric. and Mech. Coll., Stillwater, Okla. 
Dias, C. L. da S. Prof., Univ. of São Paulo, São Paulo, Brazil. 1259 W. North Shore Ave., Chicago 26, III.

Dias Coimbra, Alberto. Prof., Lyceum, Evora, Portugal. 126-20 Combatentes da Grande Guerra Rd., Coimba, Portugal.

Diaz, J. B. Asst. Prof., Brown Univ., Providence 12, R.I. 214 Laurens St., Cranston 10, R.I.

Dice, W. F. Instr., St. Francis Co11., Brooklyn 2, N.Y. 991 E. 25th St., Paterson, N.J.

Dickerson, B. K Instr, Univ. of the South, Sewanee, Tenn.

Dickinson, D. J. Teaching Fellow, Univ. of Michigan, Ann Arbor, Mich.

Dickson, L. E. Prof. Emeritus, Univ. of Chicago, Chicago 37, I1l. Care of Higinbotham, R.F.D. 2, Joliet, I11.

DiDomenica, E. M. Instr., Drexel Inst. of Tech., Philadelphia 4, Pa. 2036 S. 13th St., Philadelphia 48.

Dieckmann, R. C. H. Asso. Prof., San Jose State Coll., San Jose 14, Calif.

Dietrich, V. E. Asst., Purdue Univ., Lafayette, Ind. FPHA 508-3 Airport Rd., West Lafayette, Ind.

Dietsche, H. J. New York Univ., New York, N.Y. 117-64 123d St., Jamaica 20, N.Y.

Dietzold, R. L. Research Math., Bell Telephone Labs., Murray Hill, N.J.

Dieudonné, J. A. Prof., Univ. of Nancy, Nancy, France. 26 St. Michel St.

Diliberto, Dr. S. P. Instr., Univ. of California, Berkeley 4, Calif.

Dilworth, R. P. Asso. Prof., California Inst. of Tech., Pasadena 4, Calif.

Dimick, C. E. Capt., U.S.C.G., retired. Melrose Lodge, Tryon, N.C.

Dimond, Sister Agnes T. Prof., Coll. of Mt. St. Joseph, Mt. St. Joseph, Ohio.

Dimsdale, Dr. Bernard. Math., Ballistic Research Labs., Aberdeen Proving Ground, $\mathrm{Md}$.

Dines, L. L. Prof. Emeritus, Carnegie Inst. of Tech., Pittsburgh 13, Pa.; Visiting Prof., Northwestern Univ., Evanston, Ill. University Club, Evanston.

Dinkines, Flora. Univ. of Chicago, Chicago 37, Il1.

Dinneen, G. P. Asst., Univ. of Wisconsin, Madison 6, Wis. 177 Monroe Park, Madison 5.

Disert, Margaret C. Dean, Wilson Coll., Chambersburg, Pa.

Dismuke, Nancy M. (Mrs. Stewart). Physicist, Oak Ridge National Lab., Oak Ridge, Tenn. 151 Georgia Ave.

Dix, C. H. Asso. Prof. of Geophysics, California Inst. of Tech., Pasadena 4, Calif.

Dixon, Genevieve H. Computer, Cornell Aeronautical Lab., Inc., Buffalo 5, N.Y. 100 Willert Park, Buffalo 4.

Dixon, W. J. Asso. Prof., Univ. of Oregon, Eugene, Ore.

Dobbie, Dr. J. M. Scientific Analyst, Operations Evaluation Group, Div. of Industrial Cooperation, Massachusetts Inst. of Tech., Cambridge 39, Mass. 3638 Vacation Lane, Arlington, Va.

Dodson, Helen W. Asso. Prof., Goucher Coll., Baltimore 18, Md. Asst. Prof, of Astronomy, Univ. of Michigan, McMath-Hulbert Observatory, Lake Angelus, Box 104, Pontiac 4, Mich.

Dolciani, Dr. Mary P. Instr., Vassar Coll., Poughkeepsie, N.Y.

Dolph, C. L. Asst. Prof., Univ. of Michigan, Ann Arbor, Mich. 310 Maynard St.

Donsker, M. D. Instr., Cornell Univ., Ithaca, N.Y.

Doob, J. L. Prof., Univ. of Illinois, Urbana, Il1.

Doole, H. P. Asst. Prof., Univ. of Nebraska, Lincoln 8, Neb.

Dorroh, J. L. Texas Coll. of Arts and Industries, Kingsville, Tex.

Dorwart, H. L. Prof., Washington and Jefferson Coll., Washington, Pa. R.F.D. 6.

Doty, L. F. Asst. Prof., Aeronautical Eng. Dept., Univ. of Cincinnati, Cincinnati 21 , Ohio.

Douglas, C. H. Bursar and Prof., Accounting Dept., Catawba Coll., Salisbury, N.C.

Douglis, Avron. Inst. for Math. and Mech., New York Univ., New York 3, N.Y. 101 Woodruff Ave., Brooklyn 26, N.Y. 
Dowker, C. H. Asso. Prof., Tufts Coll., Medford 55, Mass. Fine Hall, Princeton Univ., Princeton, N.J.

Dowker, Dr. Yael N. (Mrs. C. H.). Member, Inst. for Advanced Study, Princeton, N.J.

Downey, J. F. Pres., James F. Downey and Staff, Consulting Engs., White Plains, N.Y. 20 N. Broadway.

Downey, Rev. J. V. Instr., Regis Coll., Denver 11, Colo.

Downing, H. H. Prof., Univ. of Kentucky, Lexington 29, Ky. 214 State St., Lexington 44.

Downing, R. H. Asso. Prof., Air Forces Inst. of Tech., Wright Field, Ohio.

Downs, T. L. Asso. Prof., Washington Univ., St. Louis 5, Mo.

Doyle, Martina E. Connecticut General Life Ins. Co., 55 Elm St., Hartford 6, Conn. 316 White St.

Doyle, T. C. Asst. Prof., Dartmouth Coll., Hanover, N.H. Graduate Club.

Doyle, Worthie. Teaching Fellow, California Inst. of Tech., Pasadena 4, Calif.

Doyle, w. C. Asso. Prof., Rockhurst Coll., Kansas City 4, Mo.

Dragonette, Leila A. Instr., Univ. of Pennsylvania, Philadelphia 4, Pa. 5209 Baltimore Ave., Philadelphia 43.

Drain, N. A. Senior Asst., Bur. of Aeronautics General Representative, Central District, Wright Field, Ohio.

Dreier, Theodore. Asso. Prof., Black Mountain Coll., Black Mountain, N.C.

Dresch, Dr. F. W. Asst. Dir., Computation and Ballistics Dept., Naval Proving Ground, Dahlgren, Va.

Dresden, Arnold. Prof., Swarthmore Coll., Swarthmore, Pa. 606 Elm Ave.

Dressel, F. G. Asso. Prof., Duke Univ., Durham, N.C. 309 Frances St.

Dressler, B. B. Asst. Prof., Kent State Úniv., Kent, Ohio.

Dressler, R. F. New York Univ., New York, N.Y. 510 W. Lehigh Ave., Philadelphia 33, $\mathrm{Pa}$.

Dreyfus, Denyse M. (Mrs. Richard). 24 Ismail Pacha St., Garden City, Cairo, Egypt.

Dribin, Dr. D. M. Research Analyst, Army Dept., Washington 25, D.C. 4320 4th St. N., Arlington, Va.

Droussent, Lucien. Civil Eng., Ministry of Armament, Clermont-Ferrand, France.

Drucker, B. M. Instr., Univ. of North Carolina, Chapel Hill, N.C. Box 322.

Dubisch, Roy. Asso. Prof., Fresno State Coll., Fresno 4, Calif.

Dubreil, P. J. Prof., Univ. of Paris, Paris, France. 11 René Bazin St., Paris 16.

Duffie, Dr. J. A. H. Dawson Coll., McGill Univ., Montreal, Que., Canada.

Duffin, R. J. Asso. Prof., Carnegie Inst. of Tech., Pittsburgh 13, Pa.

Dugundji, James. Asst. Prof., Univ. of Southern California, Los Angeles 7, Calif. 1117r/4 N. Clark St., Los Angeles 46.

Duncan, Dr. D. C. East Los Angeles Junior Coll., Los Angeles 22, Calif. 5357 Brooklyn.

Duncan, D. G. Teaching Fellow, Univ. of Michigan, Ann Arbor, Mich.

Dunford, Margaret J. Instr., Wayne Univ., Detroit 1, Mich.; Teacher, Southeastern High School, Detroit 14. 5085 Chatsworth, Detroit 24.

Dunford, Nelson. Prof., Yale Univ., New Haven 11, Conn. 374 Fountain St., New Haven 15.

Dunholter, Russell. Asso. Prof., Coll. of Eng., Univ. of Cincinnati, Cincinnati 21, Ohio.

Dunkel, Otto. Asso. Prof. Emeritus, Washington Univ., St. Louis 5, Mo.

Dunsmore, C. L. Teaching Asst., Univ. of California at Los Angeles, Los Angeles 24, Calif. Unit 496, Rodger Young Village, Los Angeles 25.

Duprat, J. T. Prof., Univ. of Ottawa, Ottawa, Ont., Canada.

Durand, Janet C. 223 W. Sedgwick St., Philadelphia 19, Pa.

$\dagger$ Durand, W. F. Prof. Emeritus, Stanford Univ., Stanford University, Calif. 540 Aeronautic Lab.

Duranona $\mathrm{y}$ Vedia, Dr. Agustin. Dir., Inst. of Math., Univ. of LaPlata, La Plata, Argentina. 1235 Luis Maria Campos, Buenos Aires, Argentina.

Duren, W. L. Prof., Tulane Univ. of Louisiana, New Orleans 15, La. 3323 Joseph St.

Durfee, Walter H. Dean, Hobart Coll., Geneva, N.Y. Coxe Hall. 
Durfee, William H. Asst. Prof., Dartmouth Coll., Hanover, N.H. 9 S. Balch St.

Durst, L. K. Teaching Fellow, California Inst. of Tech., Pasadena 4, Calif.

Dushnik, Ben. Asst. Prof., Univ. of Michigan, Ann Arbor, Mich. 205 West Engineering Annex.

Duthie, W. D. Prof. of Aerology, U.S. Naval Postgrad. School, Monterey, Calif. Box 19, Del Monte, Calif.

Dutka, Jacques. Asst. Prof., Rutgers Univ., New Brunswick, N.J. 740 Gerard Ave., New York 51, N.Y.

Dvoretzky, Dr. Aryeh. Member, Inst. for Advanced Study, Princeton, N.J.

Dwork, J. S. Instr., City Coll., New York 31, N.Y. 1815 Davidson Ave., New York 53.

Dwyer, P. S. Prof., Univ. of Michigan, Ann Arbor, Mich. 640 Oxford Rd.

Dye, L. A. Asso. Prof., The Citadel, Charleston, S.C.

Dyer, S. E. Univ. of Texas, Austin 12, Tex. Box 1608, University Station.

Dyer, W. G. Instr., Iowa State Coll. of Agric. and Mech. Arts, Ames, Iowa. 410 Welch Ave.

Dyer-Bennet, John. Asst. Prof., Purdue Univ., Lafayette, Ind.

Dysart, Robert. Certified Public Accountant, 50 State St., Boston 9, Mass.

Eachus, Dr. J. J. Research Eng., Navy Dept., Washington 25, D.C. 2424 37th St. N.W., Washington 7.

Eagle, E. L. Instr., Univ. of Arkansas, Fayetteville, Ark. Box 1011.

Earl, J. M. Prof., Municipal Univ. of Omaha, Omaha 6, Neb. 528 S. 53d St.

Eason, C. R. Hilltop Radio School, 1326 U St. N.W., Washington 9, D.C.

Eaton, Dr. J. E. Instr., Queens Coll., Flushing, N.Y.; Math., Naval Research Lab., Washington 20, D.C. 67-50 B 188th St., Flushing, N.Y.

Eaves, E. D. Prof., Univ. of Tennessee, Knoxville 16, Tenn. University Station.

Eberhart, Paul. Prof., Washburn Municipal Univ. of Topeka, Topeka, Kan.

Eberlein, W. F. Asst. Prof., Univ. of Wisconsin, Madison 6, Wis.

Eberman, J. W. Senior Eng., Shell Chemical Corp., P.O. Box 2633, Houston 1, Tex. Box 183, Route 16, Houston.

Ebersole, H. N. Instr., Lafayette Coll., Easton, Pa. 627 High St.

Ebin, A. W. Pres., American Inst. of Man, 800 North Tower Ct., Chicago 11, I11.

Eckert, Dr. W. J. Dir., Pure Science Dept., International Business Machines Corp., 590 Madison Ave., New York 22, N.Y. Watson Lab., 612 W. 116th St., New York 27.

Eckmann, Beno. Prof., Swiss Federal School of Tech., Zürich, Switzerland.

Eckweiler, Dr. H. J. Chief, Optical Sec., Kollsman Instrument Div., Square D Co., 80-08 45th Ave., Elmhurst, N.Y. 42-15 81st St.

Eddy, R. P. Math., Mech. Div., Naval Ordnance Lab., White Oak, Silver Spring 19, Md. 1320 Harvard St. N.W., Washington 9, D.C.

Edington, W. E. Prof., DePauw Univ., Greencastle, Ind. E. Franklin St.

Edison, T. M. Pres., Calibron Products, Inc., 51 Lakeside Ave., West Orange, N.J. Llewellyn Park.

Edmonson, Nat. Math., Applied Physics Lab., Johns Hopkins Univ., Silver Spring, Md.

Edwards, L. P. Asst. Prof., Univ. of New Brunswick, Frederickton, N.B., Canada. Apt. 34 Alexander College.

Edwards, Myrtle. Instr., Mars Hill Coll., Mars Hill, N.C.

Edwards, R. H. Instr., Illinois Inst. of Tech., Chicago 16, I1l. 9813 S. Calhoun, Chicago 17.

Efird, Laura C. Teacher, Hugh Morrison High School, Raleigh, N.C. 506 Cutler St.

Ehlers, F. E. Research Asso., Brown Univ., Providence 12, R.I. 30 N. Court St., Providence 3.

Eiardi, Rev. A. J. Asso. Prof., Boston Coll., Chestnut Hill 67, Mass.

Eide, Margaret C. (Mrs. R. B.). Prof., State Teachers Coll., River Falls, Wis. 308 S. $2 \mathrm{~d}$ St.

Eilenberg, Samuel. Prof., Columbia Univ., New York 27, N.Y. Hamilton Hall.

Eisele, Carolyn. Instr., Hunter Coll., New York 21, N.Y. 257 W. 86th St., New York 24. 
Eisenhart, Dr. Churchill. Chief, Stat. Eng. Lab., National Bur. of Standards, Commerce Dept., Washington 25, D.C. 302 South Bldg.

†Eisenhart, L. P. Prof. Emeritus, Princeton Univ., Princeton, N.J.; Executive Officer, American Philosophical Society, 104 S. 5th St., Philadelphia 6, Pa. 25 Alexander St., Princeton.

Eisenstadt, B. J. Fellow, Univ. of Michigan, Ann Arbor, Mich. 413x $\frac{1}{2}$ Forest Ave.

Elder, C. C. Hydraulic Eng., Metropolitan Water District of Southern California, 306 W. 3d St., Los Angeles 13, Calif.

Elder, J. D. Asso. Prof., St. Louis Univ., St. Louis 3, Mo.

Elkins, T. A. Geophysicist, Gulf Research and Development Co., P.O. Drawer 2038, Pittsburgh 30, Pa.

Ellingson, H. E. State Univ. of Iowa, Iowa City, Iowa. 211 Physics Bldg.

Elliott, Dr. H. Margaret. Research Fellow, Harvard Univ., Cambridge 38, Mass. 10 Lancaster St., Cambridge 40.

Elliott, W. W. Prof., Duke Univ., Durham, N.C. Box 4221, Duke Station.

Ellis, D. B. Instr., Arkansas State Coll., Jonesboro, Ark. Box 187.

Ellis, D. O. Instr., Univ. of Missouri, Columbia, Mo.

Ellis, F. M. Asst. Prof., Youngstown Coll., Youngstown 2, Ohio. 16 W. Evergreen Ave., Youngstown 7.

Ellis, Dr. G. A. Monument Ave., Bennington, Vt.

Ellis, H. V. Instr., U.S. Military Acad., West Point, N.Y. Capt., U.S.A.

Ellis, Dr. H. W. Lect., Queen's Univ., Kingston, Ont., Canada. 139 Union St. W.

Ellis, J. A. State Univ. of Iowa, Iowa City, Iowa. Belle Fourche, S.D.

Elston, Dr. F. G. Instr., Seton Hall Coll., South Orange, N.J. 242 E. 15th St., New York 3, N.Y.

Elston, J. S. Asst. Actuary, Travelers Ins. Co., Hartford 15, Conn.

Emch, Arnold. Prof. Emeritus, Univ. of Illinois, Urbana, Ill. 1002 S. Orchard St.

Emerson, M. P. Asst., Univ. of Wisconsin, Madison 6, Wis. 1525 Adams St., Madison 5 .

Enequist, L. N. Industrial Math., Eclipse-Pioneer Div., Bendix Aviation Corp., Teterboro, N. J. 134 State St., Hackensack, N.J.

Engel, J. H. Teaching Asst., Univ. of Wisconsin, Madison 6, Wis. North Hall.

Epstein, Benjamin. Asso. Prof., Wayne Univ., Detroit 1, Mich.

Epstein, Dr. Bernard. Instr., Univ. of Pennsylvania, Philadelphia 4, Pa. College Hall.

Epstein, Joseph. Instr., Philosophy Dept., Columbia Univ., New York 27, N.Y. 410 Central Park W., New York 25.

Epstein, M. P. Columbia Univ., New York 27, N.Y. 253 W. 72d St., New York 23.

Erdélyi, Dr. Arthur. Lect., Univ. of Edinburgh, Edinburgh 1, Scotland. Visiting Prof., California Inst. of Tech., Pasadena 4, Calif. 79 St. Albans Rd., Edinburgh \#9.

Erdös, Paul. Visiting Research Prof., Univ. of Syracuse, Syracuse 10, N.Y.

Ericksen, Dr. W. S. Math., U.S. Forest Products Lab., Madison, Wis. 1924 Rowley, Madison 5.

Erickson, R. W. Mississippi State Coll. for Women, Columbus, Miss.

Erkiletian, D. H. Asst. Prof., School of Mines and Metallurgy, Univ. of Missouri, Rolla, Mo.

Erzen, C. A. Asst., Univ. of Illinois, Urbana, I11. 310 S. Prospect St., Champaign, III.

Eshleman, J. D. Asso. Prof., Hampton Inst., Hampton Institute, Va. Box 75.

Esser, Dr. M. H. M. Instr., Illinois Inst. of Tech., Chicago 16, Ill.

Esteban-Carrasco, Luis. Asst. Prof., Univ. of Madrid, Madrid, Spain.

Estill, Mary E. Instr., Univ. of Texas, Austin 12, Tex. 611 W. $22 \mathrm{~d}$ St., Austin.

Esty, T. C. Prof. Emeritus, Amherst Coll., Amherst, Mass. Care of First National Bank.

Ettlinger, H. J. Prof., Univ. of Texas, Austin 12, Tex. 3110 Harris Park Ave., Austin 22.

Eudey, M. W. 686 Santa Barbara Rd., Berkeley 7, Calif.

$\dagger$ Evans, G. C. Prof., Univ. of California, Berkeley 4, Calif.

Evans, H. B. Prof. Emeritus, Univ. of Pennsylvania, Philadelphia 4, Pa. 88 Merbrook Lane, Merion Station, $\mathrm{Pa}$. 
Evans, H. P. Prof., Univ. of Wisconsin, Madison 6, Wis. North Hall.

Evans, Jane L. Teacher, Juan Demostenes Aresemana Normal School, Santiago, Panama.

Evans, J. O. Consulting Eng., P.O. Box 153, New Orleans 3, La.

Everett, Dr. C. J. Staff Member, Los Alamos Scientific Lab., Univ. of California, Los Alamos, N.M. 1334 43d St., Los Alamos 1.

Everett, H. S. Prof., Extension Div., Univ. of Chicago, Chicago 15, Ill.

Eves, Howard. Asso. Prof., Oregon State College, Corvallis, Ore.

Ewing, G. M. Asso. Prof., Univ. of Missouri, Columbia, Mo. Engineering Bldg.

Ewing, Maurice. Asso. Prof., Geology Dept., Columbia Univ., New York 27, N.Y.; Research Asso., Woods Hole Oceanographic Inst., Woods Hole, Mass.

Ewing, Meta M. Instr., Bay City Junior Coll., Bay City, Mich. 704ז 2 N. Grant P1.

Ewy, D. J. Instr., Bethel Coll., North Newton, Kan. Box 181.

Exner, R. M. Instr., Syracuse Univ., Syracuse 10, N.Y.

Fabricius-Bjerre, F. G. Prof., Tech. Univ. of Denmark, Copenhagen K, Denmark. $P$. Bangsvej 145, Copenhagen $F$.

Fagerstrom, W. H. Asst. Prof., City Coll., New York 31, N.Y. 706 Riverside Dr.

Fair, G. M. Dean and Prof., School of Eng., Harvard Univ., Cambridge 38, Mass. Pierce Hall.

Falconer, Dr. B. L. Retired. 305 Ward St., Marlin, Tex.

Fall, John. Chief, Acquisitions Div., New York Public Lib., 4765 th Ave., New York 18, N.Y.

Falvey, Frances E. Dean of Women and Asst. Prof., James Millikin Univ., Decatur, I11.

Fan, Ky. Asst. Prof., Univ. of Notre Dame, Notre Dame, Ind.

Farah, Edison. Asst., Univ. of São Paulo, São Paulo, Brazil.

Farley, Dr. B. G. Member of Tech. Staff, Bell Telephone Labs., Murray Hill, N.J.

Farnau, E. F. Prof., Chemistry Dept., Univ. of Cincinnati, Cincinnati 21, Ohio.

Farnell, Dr. A. B. Lect., Princeton Univ., Princeton, N.J.

Farrell, E. J. Instr., Univ. of San Francisco, San Francisco 17, Calif. 2526 Gough St., San Francisco 23.

Farrell, O. J. Asso. Prof., Union Coll., Schenectady 8, N.Y. Box 78, R.F.D. 1, Stone Ridge Rd., Schenectady.

Fasenmyer, Sister M. Celine. Asst. Prof., Mercyhurst Coll., Erie, Pa.

Fass, A. L. Lect., Columbia Univ., New York 27, N.Y. 882 Beck St., New York 59.

Fay, E. A. Univ. of California, Berkeley 4, Calif. 415 S. 17th St., Richmond, Calif.

Fazel, Dr. C. S. Dir. of Development, Nitrogen Sec., Solvay Process Div., Allied Chemical and Dye Corp., Hopewell, Va.

Feather, E. H. Prof., Physics Dept., Valley Forge Military Acad., Wayne, Pa. $526 \mathrm{~W}$. Beechtree Lane.

Federer, Herbert. Asso. Prof., Brown Univ., Providence 12, R.I.

Fedorovsky, B. M. Asst. Prof., St. Joseph's Coll., Philadelphia 31, Pa. 2226 Delancey P1., Philadelphia 3.

Fehr, H. F. Prof., Teachers Coll., Columbia Univ., New York 27, N.Y. 106 Morningside Dr.

Fein, Dr. Louis. Senior Physicist, Martin-Hubbard Corp., 11 Beacon St., Boston 8, Mass. 48 Mary St., Arlington 74, Mass.

Feld, J. M. Asst. Prof., Queens Coll., Flushing, N.Y.

Feldman, Chester. Univ. of Chicago, Chicago 37, I11. 1414 E. 59th St.

Feller, William. Prof., Cornell Univ., Ithaca, N.Y.

Fenchel, Dr. Werner. Docent, Tech. Univ. of Denmark, Copenhagen K, Denmark. Sønderengen 110, Søborg, Denmark.

Fender, F. G. Prof., Rutgers Univ., New Brunswick, N.J.

Fenichel, S. A. 261 Goldsmith Ave., Newark 8, N.J.

Fenn, I. H. Prof., Polytech. Inst. of Brooklyn, Brooklyn 2, N.Y.

Ferester, A. H. Instr., Trenton Junior Coll., Trenton 8, N.J. 1575 Townsend Ave., New York 52, N.Y. 
Ferguson, W. A. Asst. Prof., Univ. of Illinois, Urbana, Ill. 253 Mathematics Bldg.

Ferlini, Alvaro. Instr., St. Joseph Coll., West Hartford 7, Conn.

Ferns, H. H. Prof., Univ. of Saskatchewan, Saskatoon, Sask., Canada.

†Ferry, F. C. Pres. Emeritus, Hamilton Coll., Clinton, N.Y. 324 Hart St., New Britain, Conn.

Feyerherm, A. M. Northwest Missouri State Teachers Coll., Maryville, Mo. Fialkow, A. D. Asso. Prof., Polytech. Inst. of Brooklyn, Brooklyn 2, N.Y.

Ficken, F. A. Prof., Univ. of Tennessee, Knoxville 16, Tenn.; Consultant, Carbide and Carbon Chemicals Corp., Oak Ridge, Tenn.

Field, Peter. Prof. Emeritus, Univ. of Michigan, Ann Arbor, Mich. 2009 Vinewood Blvd.

Finan, E. J. Prof., Catholic Univ. of America, Washington 17, D.C. 604 Girard St. N.E.

Finch, J. V. Univ. of Chicago, Chicago 37, I11. 5704 S. Kimbark Ave.

Findlay, William. Prof. Emeritus, McMaster Univ., Hamilton, Ont., Canada. 34 S. Oval.

Fine, L. A. 75 Park Ter. E., New York 31, N.Y.

Fine, N. J. Asst. Prof., Univ. of Pennsylvania, Philadelphia 4, Pa. 3425 Powelton Ave.

Finkbeiner, D. T. Asst., California Inst. of Tech., Pasadena 4, Calif. 200 E. Avenue 42, Los Angeles 31, Calif.

Finston, Morton. Staff Member, Operations Evaluation Group, Div. of Industrial Cooperation, Massachusetts Inst. of Tech., Cambridge 39, Mass. 33-406.

Firestone, Dr. C. D. Instr., Rutgers Univ., New Brunswick, N.J.

Fischer, C. H. Asso. Prof., Univ. of Michigan, Ann Arbor, Mich. 3016 Angell Hall.

Fischer, Emanuel. Tutor, City College, New York 31, N.Y. 9210 69th Ave., Flushing, N.Y.

Fischer, Rev. F. J. Instr., DePaul Univ., Chicago 1, Ill. 1010 Webster Ave., Chicago 14.

Fischer, Irwin. City Coll., New York 31, N.Y. 1058 Manor Ave., New York 59.

Fischer, I. C. Asst. Prof., Eng. Dept., Univ. of Minnesota, Minneapolis 14, Minn. $150 \mathrm{Nicholson} \mathrm{Hall.}$

Fishback, W. T. Harvard Univ., Cambridge 38, Mass. 40 Kirkland St.

Fisher, Dr. F. G. Math., Navy Electronics Lab., San Diego 52, Calif. 1156 Catalina Blvd., San Diego 7.

Fisher, H. A. Prof., State Coll. of Agric. and Eng., Univ. of North Carolina, Raleigh, N.C. State College Station.

†Fite, W. B. Prof. Emeritus, Columbia Univ., New York 27, N.Y. 44 Morningside Dr., New York 25.

Fithian, J. H. Prof., Newark Coll. of Eng., Newark 2, N.J. 373 William St., East Orange, N.J.

Fitzpatrick, Robert. Lect., Univ. of Pittsburgh, Pittsburgh 13, Pa. 339 N. Aiken Ave., Pittsburgh 6.

Flanders, D. A. Senior Math., Argonne National Lab., P.O. Box 5207, Chicago 80, III.

Flanders, Harley. Fellow, Univ. of Chicago, Chicago 37, I11.

Fleiger, A. G. Research Eng., Detroit Edison Co., 2000 2d Ave., Detroit 26, Mich. 13193 Wisconsin Ave., Detroit 4.

Fleisher, Edward. Prof., Brooklyn Coll., Brooklyn 10, N.Y.

Fleming, Sarah G. Univ. of Wisconsin, Madison 6, Wis. 12 Langdon St., Madison 3.

Fleming, Walter. Ft. Hays Kansas State Coll., Hays, Kan.

Flexner, Dr. W. W. Senior Stat., United Nations, Lake Success, N.Y. Statistical Office.

Fleshler, A. D. Asst. Prof., Champlain Coll., Plattsburg, N.Y. Bldg. 77.

Flodin, I. A. Eng., American Smelting and Refining Co., San Luis Potosi, S.L.P., Mexico. Room 66.

Flood, Dr. M. M. Executive Sec., American Stat. Assn., 1603 K St. N.W., Washington 6, D.C. 3806 Kanawha St. N.W., Washington 15. 
Florio, J. S. Instr., Univ. of La Plata, La Plata, Argentina. 527 44th St.

Floris, Athanasius. Civil Eng. 748 S. Kingsley Dr., Los Angeles 5, Calif.

Floyd, E. E. Instr., Univ. of Virginia, Charlottesville, Va. 15 Monroe Apts.

Fobes, M. P. Prof., Coll. of Wooster, Wooster, Ohio.

Fog, David. Prof., Royal Veterinarian and Agric. School, Copenhagen V, Denmark. Travervänget 18 , Charlottenlund, Denmark.

Folley, K. W. Prof., Wayne Univ., Detroit 1, Mich. 19230 Gainsboro, Detroit 23.

F $\varnothing$ lner, Erling. Asst. Prof., Math Inst., Copenhagen $\varnothing$, Denmark. Almindingen 64, Søborg, Copenhagen.

Folz, C. H. Actuary, Western and Southern Life Ins. Co., 400 Broadway, Cincinnati 1, Ohio.

Foote, J. R. Instr., Massachusetts Inst. of Tech., Cambridge 39, Mass. Room 2-234.

Foote, R. T. Instr., Pennsylvania State Coll., State College, Pa.

Ford, L. R. Prof., Illinois Inst. of Tech., Chicago 16, I11.

$\uparrow$ Ford, W. B. Prof. Emeritus, Univ. of Michigan, Ann Arbor, Mich. Hayt Corners, N.Y.

Forder, H. G. Prof., University Coll., Auckland, New Zealand.

Foreman, W. C. Instr., Univ. of Kansas, Lawrence, Kan. Apt. 7-c, Sunnyside.

Forsythe, Dr. G. E. Math., Inst. for Numerical Analysis, National Bur. of Standards, Univ. of California at Los Angeles, Los Angeles 24, Calif. 944 Euclid St., Santa Monica, Calif.

Fort, Dr. M. K. Instr., Univ. of Illinois, Urbana, I11. 806 W. Clark St. Champaign, I11.

Fort, Tomlinson. Prof., Univ. of Georgia, Athens, Ga. Leconte Hall.

Foster, A. L. Asso. Prof., Univ. of California, Berkeley 4, Calif.

Foster, J. F. Instr., Univ. of Arizona, Tucson, Ariz.

Foster, M. C. Prof., Wesleyan Univ., Middletown, Conn. 30 Gordon P1.

Foster, R. M. Prof., Polytech. Inst. of Brooklyn, Brooklyn 2, N.Y. 136 Stanmore P1., Westfield, N.J.

Fostvedt, T. M. Lect., Univ. of Alberta, Edmonton, Alta., Canada.

Fouch, R. S. Instr., Univ. of Chicago, Chicago 37, I11. Faculty Exch.

Fowler, F. H. Project Eng., Lessells and Associates. 108 Washington St., Brighton 35, Mass.

Fowler, K. A. Teaching Fellow, Univ. of Michigan, Ann Arbor, Mich. 52 Donaldson Rd., Buffalo 8, N.Y.

Fowler, Sister M. Charlotte. Prof., Nazareth Coll., Louisville 3, Ky.

Fox, A. H. Asso. Prof., Union Coll., Schenectady 8, N.Y. 1101 Millington Rd.

Fox, Mother Ethelyn. Dir. of Humanities, Duchesne Coll., Omaha 3, Neb.

Fox, H. H. Asst., Univ. of Illinois, Urbana, I1l. 609 E. Washington St., Monticello, Ill.

Fox, R. H. Asso. Prof., Princeton Univ., Princeton, N.J. Fine Hall.

Foyle, Grace A. (Mrs. W. R.). 8 Gilboa St., East Douglas, Mass.

Fraenkel, A. A. Dir., Inst. of Math., and Prof., Hebrew Univ., Jerusalem. Beth Hama'aloth.

Frame, J. S. Prof., Michigan State Coll., East Lansing, Mich.

Franck, Abraham. Instr., Univ. of Minnesota, Minneapolis 14, Minn. Room 208, Main Engineering Bldg.

Frank, Evelyn. Asst. Prof., Univ. of Illinois, Chicago 11, I11. 2226 Sherman Ave., Evanston, I11.

Frankel, E. T. Budget Analyst, Federation of Social Agencies of Pittsburgh and Allegheny County, 519 Smithfield St., Pittsburgh 22, Pa.

Franken, P. A. 2 Sutton Sq., New York 22, N.Y.

Franklin, Constance W. (Mrs. Philip). Math., Harvey Radio Labs., 447 Concord Ave., Cambridge 38, Mass. 312 Pleasant St., Belmont 78, Mass.

Franklin, Philip. Prof., Massachusetts Inst. of Tech., Cambridge 39, Mass. 312 Pleasant St., Belmont 78, Mass.

Fraser, W. C. G. Asst. Prof., Rensselaer Polytech. Inst., Troy, N.Y.

Free, A. C. Actuarial Clerk, United Benefit Life Ins. Co., 33d and Farnam St., Omaha 3, Neb. 421 N. 31st St.

Free, N. S. Teaching Asst., Univ. of California, Berkeley 4, Calif. 
Freeman, J. C. Research Asst., Grad. Div. of Applied Math., Brown Univ., Providence 12, R.I. 6701 45th St., Chevy Chase 15, Md.

Freeman, M. F. Research Asst., Princeton Univ., Princeton, N.J. Graduate Coll. Freilich, Gerald. Brown Univ., Providence 12, R.I. 1235 E. 13th St., Brooklyn 30, N.Y.

Freire, L. B. Prof., Physics Dept., Univ. of Recife, Recife, Brazil. 621 Amelia St.

Freund, J. E. Asso. Prof., Alfred Univ., Alfred, N.Y. P.O. Box 182.

Freundlich, Dr. Marianne R. (Mrs. Lloyd Smith). Lect., Univ. of California, Berkeley 4, Calif.

Frey, E. J. Instr., Massachusetts Inst. of Tech., Cambridge 39, Mass. 11 Everett St., Cambridge 38.

Friedman, Bernard. Asst. Prof., New York Univ., New York, N.Y. 55 Hilltop Ave., New Rochelle, N.Y.

Friedrichs, K. O. Prof., Inst. for Math. and Mech., New York Univ., New York 3, N.Y. 157 Mt. Joy P1., New Rochelle, N.Y.

Friend, Helen L. Instr., Coe Coll., Cedar Rapids, Iowa.

Frink, Aline H. (Mrs. Orrin). Asst. Prof., Pennsylvania State Coll., State College, Pa. 706 Sunset Rd.

Frink, Orrin. Prof., Pennsylvania State Coll., State College, Pa. 706 Sunset Rd.

Frisch, Sister M. Elizabeth. Prof., Villa Madonna Coll., Covington, Ky.

Froseth, Alice J. Instr., Univ. of Illinois, Chicago 11, Ill. 5042 N. Wolcott Ave., Chicago 40.

Fry, Cleota G. Asst. Prof., Purdue Univ., Lafayette, Ind.

Fry, Dr. T. C. Dir. of Switching Research and Eng., Bell Telephone Labs., Inc., 463 West St., New York 14, N.Y.

Fry, W. J. 907 W. Eureka St., Champaign, I11.

Fryer, K. D. Fellow, Univ. of Toronto, Toronto 5, Ont., Canada. 6 Beverley St., St. Thomas, Ont.

Fuchs, Dr. W. H. J. Lect., Univ. of Liverpool, Liverpool 3, England. Visiting Asso. Prof., Cornell Univ., Ithaca, N.Y.

Fudge, Dr. Helen G. Teacher, Holmes Junior High School, Philadelphia 39, $\mathrm{Pa}$. Rosemont, $\mathrm{Pa}$.

Fulkerson, D. R. Asst., Univ. of Wisconsin, Madison 6, Wis. North Hall.

Fulks, Watson. Instr., Univ. of Minnesota, Minneapolis 14, Minn. 2012 26th Ave. S., Minnesota 6.

Fuller, Dr. D. L. Asso. Dir., Central Research Lab., General Aniline and Film Corp., Easton, $\mathrm{Pa}$.

Fuller, L. E. Asst., Univ. of Wisconsin, Madison 6, Wis. North Hall.

Fullerton, R. E. Asst. Prof., Univ. of Wisconsin, Madison 6, Wis.

Fulmer, H. K. Prof., Georgia School of Tech., Atlanta 1, Ga.

Fulton, A. E. Asst. Prof., Georgia School of Tech., Atlanta 1, Ga.

Fulton, Dr. C. M. Inst., Coll. of Agric., Univ. of California, Davis, Calif.

Fulton, D. G. Asso. Prof., Tufts Coll., Medford 55, Mass. 45 Sawyer Ave.

Fulton, L. M. Duke Univ., Durham, N.C. Box 4856, Duke Station.

Funk, Paul. Prof., Tech. Univ., Vienna IV, Austria.

Furman, Albert. Asst. Prof., Kansas State Coll. of Agric. and Applied Science, Manhattan, Kan.

Furquim de Almeida, Fernando. Prof., Univ. of São Paulo, São Paulo, Brazil. 286 Alfreda Ellis St.

Gaalswyk, Arie. Univ. of California at Los Angeles, Los Angeles 24, Calif. $4060 \mathrm{x} / 2$ Beethoven St., Venice, Calif.

Gaba, M. G. Prof., Univ. of Nebraska, Lincoln 8, Neb. 2755 Rathbone Rd., Lincoln 2.

Gaddum, J. W. Asst., Univ. of Wisconsin, Madison 6, Wis. 925 Conklin P1., Madison 5.

Gaffney, M. P. Research Asst., Univ. of Chicago, Chicago 37, I11. 525 Ash St., Winnetka, Ill.

Gage, W. H. Dean of Administrative and Inter-Faculty Affairs, Univ. of British Columbia, Vancouver, B.C., Canada.

Gaines, R. E. Prof., Univ. of Richmond, University of Richmond, Va. 
Galbraith, Dr. A. S. Math., Aberdeen Proving Ground, Md. 31 Defense Dr., Aberdeen, Md.

Gale, A. S. Prof. Emeritus, Univ. of Rochester, Rochester 3, N.Y. 93 Bellevue Dr., Rochester 7.

Gale, David. Teaching Asst., Princeton Univ., Princeton, N.J.

Gallagher, Rev. E. H. Asst. Prof., Providence Coll., Providence 8, R.I.

Galler, B. A. Teaching Asst., Univ. of California at Los Angeles, Los Angeles 24, Calif.

Galliher, H. P. Yale Univ., New Haven 11, Conn. Woodbridge, R.F.D. 2, New Haven 15.

Galper, Marcos. 105 Ipiranga St., Rio de Janeiro, Brazil.

Galuten, Aaron. Instr., Rutgers Univ., New Brunswick, N.J. 330 Wadsworth Ave., New York 33, N.Y.

Garabedian, C. A. Prof., Wheaton Coll., Norton, Mass.

Garabedian, H. A. 2d Vice Pres., John Hancock Mutual Life Ins. Co., 197 Clarendon St., Boston 16, Mass.

Garabedian, Dr. H. L. Chief, Research Reactors Sec., Div. of Research, Atomic Energy Comm., Washington 25, D.C.

Garabedian, Dr. P. R. National Research Fellow, Stanford Univ., Stanford University, Calif.

García, Mariano. Prof., Coll. of Agric. and Mech. Arts, Univ. of Puerto Rico, Mayagüez, Puerto Rico.

Gårding, Dr. Lars. Lund Univ., Lund, Sweden. Bokbindaregatan 12A.

Gardner, C. S. Math., Control Instrument Co., 67 35th St., Brooklyn 32, N.Y. 259 W. 12th St., New York 14, N.Y.

Gardner, R. S. Instr., Ohio State Univ., Columbus 10, Ohio. 215 W. 11th Ave., Columbus 1.

Garfin, Dr. Louis. Actuary, Ins. Dept., State of Oregon, State Office Bldg., Salem, Ore.

Garis, C. F. F. Prof. Emeritus, Union Coll., Schenectady 8, N.Y. 1517 Balltown Rd.

Garrahan, Sister M. Immaculata. Instr., Coll. Misericordia, Dallas, Pa.

Garrett, Dr. G. A. Head, Theoretical Analysis Dept., Carbide and Carbon Chemicals Corp., P.O. Box P, Oak Ridge, Tenn. 108 Moylan Lane.

Garrett, R. L. Prof., Athens Coll., Athens, Ala. 314 E. Bryan St.

Garrison, G. N. Asst. Prof., City Coll., New York 31, N.Y. Hudson View Gardens, 183d St. and Pinehurst Ave., New York 33.

Garvin, Sister M. Cleophas. Prof., Notre Dame Coll., South Euclid 21, Ohio.

Gaskell, R. E. Asst. Prof., Iowa State Coll. of Agric. and Mech. Arts, Ames, Iowa.

Gaspar, F. L. Dir., Inst. of Applied Math., and Prof., Univ. of Rosario, Rosario, Argentina. 2121 San Juan St.

Gassensmith, Rev. F. M. Asso. Prof., Univ. of Notre Dame, Notre Dame, Ind.

Gatewood, B. E. Asso. Prof. of Mech., Air Forces Inst. of Tech., Wright Field, Ohio. 919 Hampshire Rd., Dayton 9.

Gauthier, Abel. Prof., Univ. of Montreal, Montreal 26, Que., Canada.

Gauthier, L. E. N. Prof., Univ. of Nancy, Nancy, France. 52 Leopold Dr.

Gavurin, L. L. Lect., Columbia Univ., New York 27, N.Y. 107 Remsen Ave., Brooklyn 12, N.Y.

Geckler, R. D. Eng., Aerojet Eng. Corp., Azusa, Calif. 535 W. Bayless St.

Gehman, H. M. Prof., Univ. of Buffalo, Buffalo 14, N.Y. 163 Winspear Ave., Buffalo 15.

Geiringer, Hilda. Prof., Wheaton Coll., Norton, Mass.

Gelbart, Abe. Asso. Prof., Syracuse Univ., Syracuse 10, N.Y.

Gelbaum, B. R. Asst. Prof., Univ. of Minnesota, Minneapolis 14, Minn.

Gentry, C. B. Prof., Oakwood Coll., Huntsville, Ala.

Gentry, F. C. Asso. Prof., Arizona State Coll., Tempe, Arizona. 1109 Maple Ave.

Gentry, I. C. Duke Univ., Durham, N.C. 2608 Elgin St.

George, Dr. T. S. 300 Twining Rd., Oreland, Pa.

Gephart, Landis. Math., Air Forces. 449 Lexington St., Waltham 54, Mass. 
Gere, B. H. Asso. Prof., Hamilton Coll., Clinton, N.Y.

Gerende, L. J. Research Asst., Naval Medical Research Inst., Bethesda 14, Md. Gergen, J. J. Prof., Duke Univ., Durham, N.C. P.O. Box 4771, Duke Station.

Gerhardt, Henry. Instr., Mobile Center, Univ. of Alabama, Mobile, Ala., 1215 Elmira St.

Germond, Dr. H. H. Research Scientist, Rand Corp., Douglas Aircraft Co., 1500 4th St., Santa Monica, Calif.

Gerst, Rev. F. J. Prof., Loyola Univ., Chicago 26, Ill.

Gerst, Dr. Irving. Math., Control Instrument Co., 67 35th St., Brooklyn 32, N.Y. 1055 Clarkson Ave., Brooklyn 12.

Getchell, Dr. B. C. Research Analyst, Army Dept., Washington 25, D.C. 903 N. Wayne St., Arlington, Va.

Ghent, K. S. Asst. Prof., Univ. of Oregon, Eugene, Ore. $2105 \mathrm{McMillan}$ St.

Gibbens, Gladys E. C. Asso. Prof., Univ. of Minnesota, Minneapolis 14, Minn. 121 Folwell Hall.

Gibson, J. C. Asst., Yale Univ., New Haven 11, Conn. 208 Whitney Ave., New Haven 10.

Gibson, R. W. Prof., Physics Dept., William Penn Coll., Oskaloosa, Iowa.

Giddings, H. A. Asso. Prof., Univ. of New Hampshire, Durham, N.H. 13 Madbury Rd.

Giese, Dr. J. H. Math., Ballistic Research Labs., Aberdeen Proving Ground, Md. 667 Green St., Havre de Grace, Md.

Giever, Dr. J. B. Instr., Boston Univ., Boston 16, Mass. 166 Fuller St., Brookline 46, Mass.

Gilbarg, David. Asst. Prof., Indiana Univ., Bloomington, Ind.

Gilbert, E. N. Member of Tech. Staff, Bell Telephone Labs., Inc., 463 West St., New York 14, N.Y. 8529 89th St., Jamaica 21, N.Y.

Gilbert, Norma M. (Mrs. E. E.). Asst. Prof., Upsala Coll., East Orange, N.J. 235 Wooster St., New York 12, N.Y.

Gilbert, P. T. Research Chemist, National Tech. Labs., 820 Mission St., South Pasadena, Calif. 514 Orange Grove Ave.

Gilbert, P. W. Asst. Prof., Syracuse Univ., Syracuse 10, N.Y.

Gilbert, Shirley A. (Mrs. W. M.). Editorial Asst., Princeton Univ., Princeton, N.J. Fine Hall.

Gilbert, W. M. Instr., Princeton Univ., Princeton, N.J. Fine Hall.

Gilchrist, Lachlan. Asso. Prof., Physics Dept., Univ. of Toronto, Toronto 5, Ont., Canada.

Gildea, R. A. J. Brown Univ., Providence 12, R.I. 290 Eliot St., Milton 87, Mass.

Gill, B. P. Prof., City Coll., New York 31, N.Y. 493 Warwick Ave., West Englewood, N.J.

Gill, P. J. Asst. Seismologist, Magnetic Observ., Botanical Gardens, Christ Church, New Zealand. 131 Canon St., Christ Church N1.

Gillam, B. E. Prof., Drake Univ., Des Moines 11, Iowa.

Gillis, Dr. Joseph. 14 Thornhill Park, Sunderland, England.

Gillis, M. E. Prof., Blue Mountain Coll., Blue Mountain, Miss.

Gillman, Leonard. Staff Member, Operations Evaluation Group, Div. of Industrial Cooperation, Massachusetts Inst. of Tech., Cambridge 39, Mass. 3416 10th P1. S.E., Washington 20, D.C.

Gilman, R. E. Asso. Prof., Brown Univ., Providence 12, R.I. 44 E. Manning St., Providence 6.

Gingerich, Dr. H. F. Math., Navy Dept., Washington 25, D.C. 4607 Connecticut Ave. N.W., Washington 8.

Ginsburg, Jekuthiel. Prof., Yeshiva Univ., New York 33, N.Y. 610 W. 139th St., New York 31.

Girshick, M. A. Prof., Stat. Dept., Stanford Univ., Stanford University, Calif.

Gittings, H. T. Box 1663, Los Alamos, N.M.

Givens, Wallace. Prof., Univ. of Tennessee, Knoxville 16, Tenn.

Glazier, Harriet E. Asst. Prof. Emeritus, Univ. of California at Los Angeles, Los Angeles 24, Calif. 1307 Lucile Ave., Los Angeles 26.

Gleason, A. M. Fellow, Harvard Univ., Cambridge 38, Mass. A-25 Winthrop House. 
Gleason, R. E. Prof., Temple Univ., Philadelphia 22, Pa.

Glenn, Dr. O. E. 127 McKinley Ave., Lansdowne, Pa.

Glenn, W. H. Chairman, Natural Science Div., John Muir Junior Coll., Pasadena 3, Calif. 1425 Beech St., South Pasadena, Calif.

Glover, I. E. Prof., Langston Univ., Langston, Okla. P.O. Box 402.

Glusman, Sidney. Instr., Seton Hall Coll., South Orange, N.J. 7 W. 8th St., New York 11, N.Y.

Goa, Dr. C. S. Building Dir., Ministry of Public Works, Caracas, Venezuela. Jardines Del Valle, 4 16th St.

Gödel, Dr. Kurt. Permanent Member, Inst. for Advanced Study, Princeton, N.J.

Godfrey, E. L. Asst. Prof., Defiance Coll., Defiance, Ohio. 125 E. Sessions Ave.

Goedicke, Victor. Asso. Prof., Ohio Univ., Athens, Ohio.

Goff, J. A. Dean, Towne Scientific School, Univ. of Pennsylvania, Philadelphia 4, Pa. 109 Engineering Bldg.

Goffman, Casper. Asso. Prof., Univ. of Oklahoma, Norman, Okla.

Goheen, H. E. Asst. Prof., Syracuse Univ., Syracuse 10, N.Y. 259 Fenway Dr., Syracuse 3.

Gokhale, Dr. V. D. Lect., Rutgers Univ., New Brunswick, N.J.

Gold, J. S. Prof., Bucknell Univ., Lewisburg, Pa. 306 S. 3d St.

Goldberg, Michael. Head Eng., Bur. of Ordnance, Navy Dept., Washington 25, D.C. 5823 Potomac Ave. N.W., Washington 16.

Goldberg, Samuel. Cornell Univ., Ithaca, N.Y.

Goldhaber, J. K. Asst., Univ. of Wisconsin, Madison 6, Wis. North Hall.

Goldman, C. C. Instr., Univ. of Cincinnati, Cincinnati 21, Ohio. R.F.D. 1, Mount Orab, Ohio.

Goldman, I. L. Lect., Columbia Univ., New York 27, N.Y. 107 Broadway, Hicksville, N.Y.'

Goldman, Dr. Oscar. Instr., Harvard Univ., Cambridge 38, Mass.

Goldstein, Leon. Staff Member, Operations Evaluation Group, Div. of Industrial Cooperation, Massachusetts Inst. of Tech., Cambridge 39, Mass. 3902 53d P1., Bladensburg, Md.

Goldstein, Dr. Louis. Staff Member, Los Alamos Scientific Lab., Univ. of California, P.O. Box 1663, Los Alamos, N.M.

Goldstein, Max. Scientist, Los Alamos Scientific Lab., Univ. of California, P.O. Box 1663, Los Alamos, N.M.

Goldstine, Dr. H. H. Asst. Project Dir., Electronic Computer Project, Inst. for Advanced Study, Princeton, N.J.

Goldsworthy, E. C. Asst. Prof., California Inst. of Tech., Pasadena 4, Calif. 1245 Arden Rd., Pasadena 5.

Golomb, Michael. Asso. Prof., Purdue Univ., Lafayette, Ind.

Gomes, A. M. Asst., Univ. of Brazil, Rio de Janeiro, Brazil.

Gomide, Elza F. Asst., Univ. of São Paulo, São Paulo, Brazil.

González, J. M. 815 W. Main, E1 Paso, Tex.

Gonzalez, M. O. Prof., Univ. of Havana, Havana, Cuba.

González-Baz, Enriqueta. Teacher, Preparatory School, Univ. of Mexico, Mexico, D.F., Mexico, Edimburgo 21, Colonia del Valle.

González-Domínguez, Dr. Alberto. Univ. of Buenos Aires, Buenos Aires, Argentina.

Good, R. A. Asst. Prof., Univ. of Maryland, College Park, Md.

Goode, H. H. Math., Special Devices Center, Office of Naval Research, Sands Point, N.Y. 3251 Cambridge Ave., New York 63, N.Y.

Goodier, J. N. Prof., Mech. Dept., Stanford Univ., Stanford University, Calif. 506 Mayfield Ave.

Goodman, Dr. A. W. Instr., Rutgers Univ., New Brunswick, N.J. 10 Brookside Ave., Somerville, N.J.

Goodman, Ruth E. Asst. Prof., Duquesne Univ., Pittsburgh 19, Pa.

Goodner, D. B. Univ. of Illinois, Urbana, I11. $506 \mathrm{~S}$. Mathews.

Goodrich, M. T. Asst. Prof., Keene Teachers Coll., Keene, N.H. 36 Wyman Way.

Gopaul, A. A. Asst., Univ. of Illinois, Urbana, Ill. 501 S. Wright St., Champaign, IIl.

Gorciu, V. G. Instr., Univ. of Maryland, College Park, Md. 
Gordon, R. D. Asst. Prof., Univ, of Buffalo, Buffalo 14, N.Y.

Gordon, W. O. Asso. Prof., Pennsylvania State Coll., State College, Pa.

Gore, G. D. Prof., Roosevelt Coll., Chicago 5, Ill.

Gorenstein, Daniel. Harvard Univ., Cambridge 38, Mass. 37 Commonwealth Ave., Newton, Mass.

Gormley, P. G. Prof., University Coll., Dublin, Ireland. 54 Ranelagh Rd.

Gorn, Dr. Saul. Staff Math., Aircraft Radiation Lab., Air Materiel Command, Wright Field, Ohio. 246 Niagara Ave., Dayton 5, Ohio.

Gottlieb, M. J. Asst. Prof., Newark Coll., Rutgers Univ., Newark 2, N.J.

Gottschalk, W. H. Asst. Prof., Univ. of Pennsylvania, Philadelphia 4, Pa. Box 6, College Hall.

Gotz, Dr. Wulf. Columbia Univ., New York 27, N.Y. 410 Riverside Dr., New York 25.

Gough, Sister M. de Lellis. Prof., Incarnate Word Coll., San Antonio 2, Tex.

†Gould, Alice B. Care of Samuel Vaughan, 35 Congress St., Boston 9, Mass.

Gould, S. H. Asst. Prof., Purdue Univ., Lafayette, Ind.

Gourin, Dr. Eli. Lect., Columbia Univ., New York 27, N.Y. 33 E. 208th St., New York 67.

Gourrich, G. E. Elec. Eng., National Bur. of Standards, Commerce Dept., Washington 25, D.C. 3700 Massachusetts Ave. N.W., Washington 16.

Gouwens, Cornelius. Prof., Iowa State Coll. of Agric. and Mech. Arts, Ames, Iowa.

Graber, M. E. Prof., Physics Dept., Morningside Coll., Sioux City, Iowa.

Grable, E. S. Asst. Prof., Univ. of Richmond, University of Richmond, Va. Box 45.

Grad, Arthur. Math., Math. Branch, Office of Naval Research, Washington 25, D.C. 4320 3d St. S.E., Washington 20.

Grad, Harold. Asst. Prof., New York Univ., New York 3, N.Y. 743 Kelly St., New York 55.

Graef Fernandez, Carlos. Dir., Inst. of Physics, Univ. of Mexico, Mexico, D.F., Mexico.

Graesser, R. F. Prof., Univ. of Arizona, Tucson, Ariz. 1648 E. 5th St.

Graham, J. W. Instr., Ripon Coll., Ripon, Wis.

Graham, P. H. Asso. Dean and Prof., New York Univ., New York 3, N.Y.

† Grant, Alice A. Prin., Miss Grant's School, 215 College St., Toronto, Ont., Canada.

Grant, Dr. Anna M. C. Project Eng., Sperry Gyroscope Co., Lake Success, N.Y. 185 N. Village Ave., Rockville Centre, N.Y.

Grant, H. S. Asso. Prof., Rutgers Univ., New Brunswick, N.J.

Grant, U. S. Prof., Geology Dept., Univ. of California at Los Angeles, Los Angeles 24, Calif.

Gras, E. C. Asst. Prof., U.S. Naval Acad., Annapolis, Md.

Grau, A. A. Asso. Prof., Univ. of Oklahoma, Norman, Okla.

Graustein, Mary C. (Mrs. W. C.). Asst. Prof., Tufts Coll., Medford 55, Mass. Westminster, Mass.

Gravalos, F. G. Asso. Prof., Aeronautical Eng. Dept., Rensselaer Polytech. Inst., Troy, N.Y. Ricketts Bldg.

Graves, G. H. Asso. Prof., Purdue Univ., Lafayette, Ind. 227 S. Grant St., West Lafayette, Ind.

Graves, L. M. Prof., Univ. of Chicago, Chicago 37, I11. Eckhart Hall.

Graves, R. E. Asst. Prof., Univ. of Minnesota, Minneapolis 14, Minn. Folwell Hall.

Graves, W. L. Asst. Prof., Drury Coll., Springfield, Mo. 920 S. Fremont Ave.

Gray, Fletcher. Public Accountant, Room 216, Empire Bldg., Birmingham 3, Ala.

Gray, Dr. Marion C. Member of Tech. Staff, Bell Telephone Labs., Murray Hill, N.J.

Greeley, J. B. Asst. Prof., Utica Coll., Syracuse Univ., Utica, N.Y.

Green, Dorothy E. Huntingdon Coll., Montgomery, Ala. 1206 S. Court St.

Green, Dr. F. W. 6203 Washington Ave., St. Louis 5, Mo.

Green, J. W. Asso. Prof., Univ. of California at Los Angeles, Los Angeles 24, Calif. 
Green, L. J. Asst. Prof., Case Inst. of Tech., Cleveland 6, Ohio.

Greenberg, H. J. Asst. Prof., Brown Univ., Providence 12, R.I.

Greene, Laura Z. Asst. Prof., Washburn Municipal Univ. of Topeka, Topeka, Kan. 1516 Boswell.

Greene, R. L. Student Eng., Westinghouse Elec. Corp., East Pittsburgh, Pa. 210 S. Dullar Ave., Pittsburgh 8, Pa.

Greenspan, Bernard. Asst. Prof., Drew Univ., Madison, N.J. 48 Broadway, Florham Park, N.J.

Greenspan, Harold. Asst., Illinois Inst. of Tech., Chicago 16, Ill. 1026 N. Kedzie Ave., Chicago 51.

Greenstone, Dr. Leonard. Instr., Univ. of California at Los Angeles, Los Angeles 24, Calif.

Greenwood, R. E. Asst. Prof., Univ. of Texas, Austin 12, Tex. 1704 Windsor Rd., Austin.

Greer, Edison. Asso. Prof., Kansas State Coll. of Agric. and Applied Science, Manhattan, Kan. 1604 Pierre.

Greer, E. V. Bethany-Peniel Coll., Bethany, Okla. 700 N. Donald St.

Gregory, R. T. Iowa State Coll. of Agric. and Mech. Arts, Ames, Iowa. 851 Pammel Ct.

Greitzer, S. L. Instr., Talmudical Acad., Yeshiva Univ., New York 33, N.Y.; Teacher, Bronx High School of Science, New York 53, N.Y. 1535 Undercliff Ave., New York 53.

Grennan, Dr. Elizabeth B. (Mrs. John). 719 S. 7th St., Ann Arbor, Mich.

Greville, Dr. T. N. E. Prin. Actuarial Math., National Office of Vital Statistics, Public Health Service, Washington 25, D.C. P.O. Box 7363, Washington 4.

Griffin, F. L. Prof., Reed Coll., Portland 2, Ore.

Griffin, Harriet. Asst. Prof., Brooklyn Coll., Brooklyn 10, N.Y. 3609 Farragut Rd.

Griffith, W. C. Asso. Prof., Centenary Coll., Shreveport, La.

Griffiths, Lois W. Asso. Prof., Northwestern Univ., Evanston, I11. 220 Lunt Hall.

†Groat, B. F. Consulting Eng. 6733 Emlen St., Philadelphia 19, Pa.

Groenewoud, Cornelius. Instr., Michigan State Coll., East Lansing, Mich.

Gross, Dr. G. L. Research Eng., Grumman Aircraft Eng. Corp., Bethpage, N.Y. 78 Sammis St., Huntington, N.Y.

Grossman, E. A. Actuarial Admin., U.S. Life Ins. Co., 84 William St., New York 7, N.Y. 103 Sun Haven Dr., New Rochelle, N.Y.

Grossman, George. Teacher, De Witt Clinton High School, New York 63, N.Y.; Instr., Polytech. Inst. of Brooklyn, Brooklyn 2, N.Y. 3425 Gates P1., New York 67.

Grosswald, Emil. Instr., Univ. of Pennsylvania, Philadelphia 4, Pa. Care of Klein, 1075 Flatbush Ave., Brooklyn 26, N.Y.

Groth, A. O. Asso. Actuary, Equitable Life Ins. Co. of Iowa, Des Moines 6, Iowa.

Grove, Dr. C. C. Retired. 143 Milburn Ave., Baldwin, N.Y.

Grove, V. G. Prof., Michigan State Coll., East Lansing, Mich. 438 Rcsewood Ave.

Grover, Blanche B. (Mrs. R. M.). Asst. Prof., Univ. of Houston, Houston 4, Tex. 1032 Walling St., Houston 9.

Grünbaum, Adolf. Fellow, Yale Univ., New Haven 11, Conn. Box 2781, Yale Station.

Guenther, P. E. Asst. Prof., Case Inst. of Tech., Cleveland 6, Ohio. 1597 Westwood Ave., Lakewood, Ohio.

Guggenbuhl, Laura. Asst. Prof., Hunter Coll., New York 21, N.Y. 2685 Grand Concourse, New York 58.

Guglialmelli, J. P. San Martín 81, San Martín, Argentina.

Gumbel, E. J. Visiting Prof., New School for Social Research, New York 11, N.Y. 441 Ocean Ave., Brooklyn 26, N.Y.

†Gummere, Dr. H. V. Retired. 3026 Midvale Ave., Philadelphia 29, Pa.

Gunderson, Dr. N. G. Instr., Univ. of Rochester, Rochester 3, N.Y. River Campus. 
Gurland, John. Univ. of California, Berkeley 4, Calif. International House.

Gurney, Dr. Margaret. Sampling Stat., Bur. of the Census, Commerce Dept., Washington 25, D.C. 6102 Lombard St., Cheverly, Hyattsville, Md.

Gustin, William. Asst. Prof., Indiana Univ., Bloomington, Ind.

Gutiérrez, L. G. Asso. Prof., Univ. of Havana, Havana, Cuba.

Gutmann, Pierre. Stat., General Cable Corp., Bayonne, N.J. 530 Riverside Dr., New York 27, N.Y.

Gutterman, M. M. Univ. of Chicago, Chicago 37, I11. Room 303, Woodlawn Hall, 6208 Drexel Ave.

Gwathmey, Mary S. No address.

Habicht, Dr. W. R. 18 Fulach St., Schaffhausen, Switzerland.

Hacker, S. G. Prof., State Coll. of Washington, Pullman, Wash.

Hackett, Sister Edward J. St. Mary's Coll., Holy Cross, Notre Dame, Ind.

Hadamard, Jacques. Prof. Emeritus, Coll. of France and Polytech. School, Paris, France. 12 Emile Faguet St., Paris 14.

Hadlock, E. H. Asso. Prof., Univ. of Florida, Gainesville, Fla. 1235 Cherokee Ave.

Hafner, Sister M. Raphael. Instr., Immaculata Coll., Immaculata, Pa.

Hafner, Ralph. Instr., Univ. of Dayton, Dayton 9, Ohio. Box 123.

Hagen, Beatrice L. Asso. Prof., Pennsylvania State Coll., State College, Pa.

Haggerty, G. B. Asst. Prof., Rhode Island State Coll., Kingston, R.I. P.O. Box 199 .

Hahn, Dr. S. W. Instr., Univ. of Michigan, Ann Arbor, Mich. 1431 University Ter.

Hahnemann, Elizabeth. Tech. Asst., Connecticut Coll., New London, Conn. 832 Humboldt Pkwy., Buffalo 11, N.Y.

Hailperin, C. B. Instr., Duquesne Univ., Pittsburgh 19, Pa.

Hailperin, Theodore. Asst. Prof., Lehigh Univ., Bethlehem, Pa.

Haimo, Deborah T. (Mrs. Franklin). 6918 Mill Brook Blvd., St. Louis 5, Mo.

Haimo, Franklin. Asst. Prof., Washington Univ., St. Louis 5, Mo. 6918 Mill Brook Blvd.

Halbert, K. W. Stat., American Telephone and Telegraph Co., 195 Broadway, New York 7, N.Y.

Hald, A. H. Prof., Univ. of Copenhagen, Copenhagen $\varnothing$, Denmark. Emdrupvaenge 94, Copenhagen.

Haley, K. D. C. Asst. Prof., Acadia Univ., Wolfville, N.S., Canada.

Halfar, Edwin. Asst. Prof., Univ. of Nebraska, Lincoln 8, Neb. Room 213, Burnett Hall.

Hall, C. F. Asst. Prof., Rensselaer Polytech. Inst., Troy, N.Y. Carnegie Bldg.

Hall, D. W. Prof., Univ. of Maryland, College Park, Md. Box 162.

Hall, F. C. Asst. Prof., Manhattan Coll., New York 63, N.Y. 430 W. 119th St., New York 27.

Hall, Marshall. Prof., Ohio State Univ., Columbus 10, Ohio.

Hall, N. A. Prof., Mech. Eng. Dept., Univ. of Minnesota, Minneapolis 14, Minn. 1779 James Ave. S., Minneapolis 5.

Hall, N. S. Teaching Asst., Univ. of California, Berkeley 4, Calif. 35 Syndicate Ct. S., Harbor Gate, Richmond, Calif.

Hall, Philip. Lect., Kings Coll., Cambridge Univ., Cambridge, England.

Hallam, Sarah M. Sec., Math. Dept., Univ. of California, Berkeley 4, Calif.

Haller, Mary E. Asst. Prof., Univ. of Washington, Seattle 5, Wash.

Hallett, Dr. W. N. Deputy Chief, Reports Branch, Office of Military Govt. for Württenberg-Baden, Stuttgart, Germany. APO 154, New York, N.Y.

Halmos, P. R. Asst. Prof., Univ. of Chicago, Chicago 37, I11. Eckhart Hall.

Halperin, Israel. Asso. Prof., Queen's Univ., Kingston, Ont., Canada.

Halperin, Morris. Appraiser of Merchandise, U. S. Customs Service, 201 Varick St., New York 14, N.Y. 7 Balfour P1., Brooklyn 25, N.Y.

Halteman, A. E. Asst. Prof., Univ. of Idaho, Moscow, Idaho.

Haltiner, G. J. Asso. Prof. of Aerology, U.S. Naval Postgrad. School, Monterey, Calif. 
Hamilton, H. J. Asso. Prof., Pomona Coll., Claremont, Calif. 735 Yale.

Hamilton, O. H. Prof., Oklahoma Agric. and Mech. Coll., Stillwater, Okla. 503 Pine St.

Hammer, Dr. Carl. Instr., Walter Hervey Junior Coll., New York 23, N.Y. 141-11 68th Dr., Flushing, N.Y.

Hammer, Dr. P. C. Group Leader, Los Alamos Scientific Lab., Univ. of California, Los Alamos, N.M. 4753 Sandia Dr.

Hamming, Dr. R. W. Member of Tech. Staff, Bell Telephone Labs., Murray Hill, N.J.

Hammond, E. E. Asst., Brown Univ., Providence 12, R.I. Porter Rd., Andover, Mass.

Hammond, E. S. Dir. of Admissions and Prof., Bowdoin Coll., Brunswick, Me. 9 Thompson St.

Hamstrom, Mary-Elizabeth. Univ. of Texas, Austin 12, Tex. 2507 San Jacinto Blvd.

Handelman, G. H. Asst. Prof., Carnegie Inst. of Tech., Pittsburgh 13, Pa.

Hanneken, C. B. Asst., Univ. of Illinois, Urbana, I11. 305 E. John St., Champaign, I11.

Hansen, A. G. Math., Flight Propulsion Research Lab., National Advisory Com. for Aeronautics, Cleveland Airport, Cleveland, Ohio. 4728 Bridge Ave., Cleveland 2.

Hansen, H. H. Snorresgade $2^{5}$, Copenhagen S, Denmark.

Hansman, Margaret M. Asst. Prof., Colorado Coll., Colorado Springs, Colo. 2038 Armstrong Ave.

Harary, Dr. Frank. Instr., Univ. of Michigan, Ann Arbor, Mich.

Harder, Dr. E. L. Consulting Transmission Eng., Westinghouse Elec. Corp., East Pittsburgh, $\mathrm{Pa} .1204$ Milton Ave., Pittsburgh 18, Pa.

Hardin, R. I. Lawyer, 31 Nassau St., New York 5, N.Y.

† Hardy, J. G. Prof. Emeritus, Williams Coll., Williamstown, Mass. 6604 S.E. 38th Ave., Portland 2, Ore.

Harish-Chandra, Dr. Member, Inst. for Advanced Study, Princeton, N.J.

Harkin, Dr. Duncan. Consultant, Naval Research Lab., Washington 20, D.C. 3000 Crest Ave., Cheverly, Md.

Harp, H. G. Instr., Ohio State Univ., Columbus 10, Ohio. 243 16th Ave., Columbus 1.

Harper, F. S. Prof., Actuarial Science Dept., Drake Univ., Des Moines 11, Iowa. 8 Cole Hall.

Harrington, C. H. Instr., Glendale Coll., Glendale 8, Calif. 1330 Hillside Dr.

Harrington, W. J. Asst. Prof., Pennsylvania State Coll., State College, Pa.

Harris, I. H. Asso. Prof., Oklahoma Baptist Univ., Shawnee, Okla.

Harris, L. J. Staff Elec. Eng., Reduction Plant, Aluminum Co. of America, Alcoa, Tenn. P.O. Box 102, Maryville, Tenn.

Harris, Dr. T. E. Research Stat., Rand Corp., Douglas Aircraft Co., 3000 Ocean Park Blvd., Santa Monica, Calif.

Harris, V. C. Instr., Northwestern Univ., Evanston, I11. 1725 Orrington Ave.

Harrison, C. E. Instr., Teacher's Training Coll., Central Inst. for the Deaf, Washington Univ., St. Louis 10, Mo. 818 S. Kingshighway Blvd.

Harrison, Gerald. Asst. Prof., Wayne Univ., Detroit 1, Mich.

Harrison, M. G. Aerodynamacist, Eastman Kodak Co., Rochester 4, N.Y. 254 Sagamore Dr., Rochester 12.

Harrje, H. J. Architect and Civil Eng., P.O. Box 746, Jacksonville Beach, Fla.

Harrold, O. G. Prof., Univ. of Tennessee, Knoxville 16, Tenn.

Harshbarger, Frances. Prof., Kent State Univ., Kent, Ohio.

Hart, Bertha I. Math., Ballistic Research Labs., Aberdeen Proving Ground, Md.

Hart, W. L. Prof., Univ. of Minnesota, Minneapolis 14, Minn. 119 Folwell Hall.

Hart, W. W. Retired. Box 189, Libertyville, I11.

Harter, H. L. Fellow, Purdue Univ., Lafayette, Ind. Bldg. 9, Apt. 7, Ross-Ade Dr., West Lafayette, Ind.

Harter, N. W. Prof., Thiel Coll., Greenville, Pa.

Hartman, Philip. Asso. Prof., Johns Hopkins Univ., Baltimore 18, Md.

Hartnell, George. Retired. Wyoming, N.Y. 
Hartzler, H. H. Prof., Goshen Coll., Goshen, Ind. Steward Observatory, Univ. of Arizona, Tucson, Ariz.

Harvey, Dr. A. R. Fellow, California Inst. of Tech., Pasadena 4, Calif.

Harvey, G. G. Asso. Prof., Physics Dept., Massachusetts Inst. of Tech., Cambridge 39, Mass.

Haskins, E. E. Ásso. Prof., Fenn Coll., Cleveland 15, Ohio.

Hassler, J. O. Prof., Univ. of Oklahoma, Norman, Okla. 425 S. Lahoma.

Hastings, Cecil. Research Math., Douglas Aircraft Co., 1500 4th St., Santa Monica, Calif. 9075 th St.

Hatcher, T. W. Prof., Virginia Polytech. Inst., Blacksburg, Va. P.O. Box 172.

Hatfield, Charles. Asst. Prof., Univ. of Minnesota, Minneapolis 14, Minn. 4612 14th Ave. S., Minneapolis 7.

Hatke, Sister M. Agnes. Dean of Students and Instr., St. Francis Coll., Ft. Wayne 8, Ind.

Hattan, Dr. Corinne. Instr., Univ. of Illinois, Urbana, Ill. 153 Mathematics Bldg.

Hauser, A. A. Senior Project Eng., Sperry Gyroscope Co., Lake Success, N.Y. 36 Hamilton P1., Garden City, N.Y.

Hausmann, Rev. B. A. Prof., West Baden Coll., Loyola Univ., West Baden Springs, Ind.

Haviland, E. K. Asso. Prof., Johns Hopkins Univ., Baltimore 18, Md. Rowland Hall.

Hawkins, David. Asso. Prof., Philosophy Dept., Univ. of Colorado, Boulder, Colo.

Hawkins, Ernest. Asso. Prof., U.S. Naval Acad., Annapolis, Md. 4 Steele Ave.

Hawley, N. S. Fellow, Princeton Univ., Princeton, N.J.

Hay, G. E. Asso. Prof., Univ. of Michigan, Ann Arbor, Mich. 274 West Engineering Bldg.

Hayden, Camilla. Prof., St. Mary-of-the-Woods Coll., St. Mary-of-the-Woods, Ind.

Hayes, C. A. Asst. Prof., Coll. of Agric., Univ. of California, Davis, Calif.

Hayes, J. J. Asst. Prof., Univ. of Utah, Salt Lake City 1, Utah.

Hayes, R. M. Univ. of California at Los Angeles, Los Angeles 24, Calif. 17402 Chatsworth St., San Fernando, Calif.

Haynes, Euphemia L. (Mrs. H. A.). Asso. Prof., Miner Teachers Coll., Washington 1, D.C. 1237 Franklin St. N.E., Washington 17.

Haynes, Nola A. (Mrs. E. S.). Acting Asso. Prof., Univ. of Missouri, Columbia, Mo. 1408 Rosemary Lane.

Haywood, Stuart. Asst., Univ. of Maryland, College Park, Md. Box 81.

Hazard, C. T. Prof., Purdue Univ., Lafayette, Ind.

Hazard, Katharine E. Asst. Prof., New Jersey Coll. for Women, Rutgers Univ., New Brunswick, N.J.

Hazeltine, Alan. Tech. Consultant. 15 Tower Dr., Maplewood, N.J.

†Hazlett, Olive C. Asst. Prof., Univ. of Illinois, Urbana, I11. Care of J. O. Huff, $212 \mathrm{~W}$. Washington Ave.

H'Doubler, Dr. F. T. Surgeon, A Medical Arts Bldg., Springfield, Mo.

Heacock, R. R. Teaching Fellow, Univ. of California, Berkeley 4, Calif. 1411 Allston Way, Berkeley 2.

Heaslet, Dr. M. A. Aeronautical Research Scientist, National Advisory Com. for Aeronautics, Ames Aeronautical Lab., Moffett Field, Calif. P.O. Box 402, Los Altos, Calif.

Heath, J. M. Teacher, Hopkins Grammar School, New Haven, Conn. 25 Curtis Ave., Wallingford, Conn.

Hebbert, Dr. C. M. Member of Tech. Staff, Bell Telephone Labs., Inc., 463 West St., New York 14. N.Y.

Heckman, R. F. Vice Pres. and Chief Eng., Ingwersen Mfg. Co., Inc., 1800 S. Acoma St., Denver 10, Colo. 1725 Sherman St., Denver 5.

Hedberg, E. A. Asso. Prof., Univ. of South Carolina, Columbia 19, S.C. Sloan College.

Hedge, Dr. I. B. Research Eng., Ordnance Dept., Army Dept., Washington 25, D.C. 2921 S. Buchanan St., Fairlington, Arlington, Va. 
Hedlund, G. A. Prof., Yale Univ., New Haven 11, Conn.

Hefner, R. A. Dean, Georgia School of Tech., Atlanta 1, Ga.

Heilbronn, Dr. H. A. Reader, Univ. of Bristol, Bristol 8, England. The Royal Fort.

Heins, A. E. Asso. Prof., Carnegie Inst. of Tech., Pittsburgh 13, Pa.

Heins, M. H. Prof., Brown Univ., Providence 12, R.I.

Heinsheimer, Ruth. Asst., Univ. of Illinois, Urbana, Ill. 153 Mathematics Bldg.

Heller, Alex. Fellow, Columbia Univ., New York 27, N.Y. 315 Riverside Dr., New York 25.

Hellinger, E. D. Prof., Northwestern Univ., Evanston, I1l. 2215 Maple Ave.

Helme, G. C. Instr., Pratt Inst., Brooklyn 5, N.Y. 161 Emerson P1.

Helmer, Dr. Olaf. Research Math., Rand Corp., Douglas Aircraft Co., 1500 4th St., Santa Monica, Calif. 10468 Scenario Lane, Los Angeles 24, Calif.

Helsel, R. G. Asso. Prof., Ohio State Univ., Columbus 10, Ohio.

Helton, F. F. Prof., Central Coll., Fayette, Mo. Morrison Observatory.

Hemmingsen, Erik. Asst. Prof., Syracuse Univ., Syracuse 10, N.Y.

Henderson, Emma M. (Mrs. K. W.). Teaching Asst., Univ. of Southern California, Los Angeles 7, Calif. 3985 S. Vermont Ave., Los Angeles 37.

Hendrickson, M. S. Asso. Prof., Univ. of New Mexico, Albuquerque, N.M.

Henkin, Dr. L. A. Fellow, Princeton Univ., Princeton, N.J. Fine Hall.

Henriques, Anna S. (Mrs. D. E.) Asso. Prof., Univ. of Utah, Salt Lake City 1, Utah.

Herbach, L. H. Instr., Brooklyn Coll., Brooklyn 10, N.Y.

Herpel, Coleman. Asso. Prof., Undergraduate Center, Pennsylvania State Coll., Altoona, $\mathrm{Pa}$. 212 Coleridge Ave.

Herr, D. I. Executive Eng., Allen Bradley Co., Milwaukee 4, Wis.

Herr, Gertrude A. Asso. Prof., Iowa State Coll. of Agric. and Mech. Arts, Ames, Iowa. 24041/2 Knapp St.

Herrick, H. L. Asst. in instruction, Yale Univ., New Haven 11, Conn. 1543 2d St., Boone, Iowa.

Herriot, J. G. Asst. Prof., Stanford Univ., Stanford University, Calif.

Herschdorfer, Manuel. Prof., Seton Hall Coll., South Orange, N.J.

Herschfeld, Aaron. Junior Actuarial Math., Office of the Actuary, Social Security Admin., Washington 25, D.C.

Hersh, N. K. Instr., Tulane Univ. of Louisiana, New Orleans 15, La. 4919 Willow.

Hershner, I. R. Asst. Prof., Univ. of North Carolina, Chapel Hill, N.C.

Herstein, Dr. I. N. Instr., Univ. of Kansas, Lawrence, Kan.

Herzberger, Dr. M. J. Member of Research Staff, Eastman Kodak Research Lab., Rochester 4, N.Y. 186 Augustine St., Rochester 13.

Herzog, Fritz. Asso. Prof., Michigan State Coll., East Lansing, Mich.

Hess, G. W. Prof., Howard Coll., Birmingham 6, Ala. 8009 4th Ave. S.

Hestenes, Dr. A. D. Chief of Analysis Sec., Electronics and Instruments Div., Franklin Inst., Philadelphia 3, Pa. 904 Turner Ave., Drexel Hill, Pa.

Hestenes, M. R. Prof., Univ. of California at Los Angeles, Los Angeles 24, Calif.

Hester, D. M. Baker Univ., Baldwin City, Kan. 611 5th St.

Hett, Dr. J. H. Research Asso., New York Univ., New York 53, N.Y. 3140 Netherland Ave., New York 63.

Hewitt, Edwin. Asst. Prof., Univ. of Washington, Seattle 5, Wash.

Heyda, Dr. J. F. Math., Research Dept., Naval Ordnance Plant, Indianapolis, Ind.

Hibbard, Wilbur. Instr., Lehigh Univ., Bethlehem, $\mathrm{Pa}$.

Hickman, J. S. Physicist, Navy Electronics Lab., San Diego 52, Calif. 3242 Lucinda St., San Diego 6.

Hickson, A. O. Asst. Prof., Duke Univ., Durham, N. C. 2712 Legion Ave.

Higdon, Archie. Asso. Prof., Theoretical and Applied Mech. Dept., Iowa State Coll. of Agric. and Mech. Arts, Ames, Iowa. 208 M.H.

Higgins, C. H. Prof., Monmouth Junior Coll., Long Branch, N.J. 169 Chelsea Ave.

Hightower, Dr. Ruby U. Retired. Quitman, Ga. 
Hildebrand, F. B. Asst. Prof., Massachusetts Inst. of Tech., Cambridge 39, Mass. Hildebrand, J. L. Asso., Univ. of Washington, Seattle 5, Wash.

Hildebrandt, E. H. C. Asso. Prof., Northwestern Univ., Evanston, I11.

Hildebrandt, T. H. Prof., Univ. of Michigan, Ann Arbor, Mich. 1930 Cambridge Rd.

Hilding, Dr. S. H. Instr., Univ. of Stockholm, Stockholm, Sweden. Hallebergsv. 13, Traneberg, Sweden.

Hildner, R. C. Asso. Prof., Univ. of New Mexico, Albuquerque, N.M. Box 16.

Hill, C. G. Senior Partner, Cyrus G. Hill, Engineers, 231 LaSalle St., Chicago 4, I11.

Hill, E. L. Prof., Physics Dept., Univ. of Minnesota, Minneapolis 14, Minn.

Hill, J. D. Asso.' Prof., Michigan State Coll., East Lansing, Mich.

Hill, L. S. Asso. Prof., Hunter Coll., New York 21, N.Y. 22 Sagamore Rd., Bronxville 8, N.Y.

Hill, Sister M. Laetitia. Prof., Our Lady of the Lake Coll., San Antonio 7, Tex.

Hill, Winifred A. Instr., Alabama Polytech. Inst., Auburn, Ala. 271 S. Gay St.

Hille, Einar. Prof., Yale Univ., New Haven 11, Conn. 72 Edgehill Rd.

Hillman, A. P. Asso. Math., Computation Lab., National Bur. of Standards, 150 Nassau St., New York 7, N.Y. 1455 Sheridan Ave., New York 57.

Hilsenrath, Joseph. Instruction Unit Head, Training Div., Naval Ordnance Lab., Washington 25, D.C. 601 Erie Ave., Takoma Park 12, Md.

Hinman, H. H. Instr., City Coll., New York 31, N.Y.

Hinrichsen, J. J. L. Asso. Prof., Iowa State Coll. of Agric. and Mech. Arts, Ames, Iowa.

Hirsch, W. M. Inst. for Math. and Mech., New York Univ., New York 3, N.Y. 2791 University Ave., New York 63.

Hirschman, Dr. I. I. Fellow, Harvard Univ., Cambridge 38, Mass. 42 Kinnaird St., Cambridge 39.

Hizon, M. O. Actuary-Examiner, Bur. of Banking, Manila, Philippines.

Hlavatý, Vaclav. Visiting Prof., Indiana Univ., Bloomington, Ind.

Hobbs, A. W. Dean, Coll. of Arts and Sciences, Univ. of North Carolina, Chapel Hill, N.C.

Hochschild, G. P. Asst. Prof., Univ. of Illinois, Urbana, I11.

Hodell, L. R. Creole Petroleum Corp., Caracas, Venezuela.

Hodge, F. H. Retired. 1010 S. 21st St., Terre Haute, Ind.

Hodges, J. L. Teaching Asst., Univ. of California, Berkeley 4, Calif. 3244 California St., Berkeley 3.

Hodgkinson, William. Stat., American Telephone and Telegraph Co., 195 Broadway, New York 7, N.Y.

Hoel, P. G. Prof., Univ. of California at Los Angeles, Los Angeles 24, Calif.

Hoersch, v. A. Asst. Prof., Univ. of Illinois, Urbana I1l. 909 S. 1st St., Champaign, I11.

Hoffman, A. J. Fellow, Columbia Univ., New York 27, N.Y. 2776 Jerome Ave., New York 58.

Hoffman, W. C. 833 S. Mansfield Ave., Los Angeles 36, Calif.

Hoffmann, Banesh. Asst. Prof., Queens Coll., Flushing, N.Y. 43-17 169th St.

Hoffmann, P. O. Prof., Physics Dept., Newark Coll. of Eng., Newark, N.J.

Hohaus, R. A. Asst. Actuary, Metropolitan Life Ins. Co., 1 Madison Ave., New York 10, N.Y.

Hohn, Dr. F. E. Instr., Univ. of Illinois, Urbana, Ill.

Holdman, R. E. Box 157, Times Sq. Station, New York 18, N.Y.

von Holdt, R. E. Instr., Northwestern Ưniv., Evanston, I11. 1725 Orrington Ave.

Holl, D. L. Prof., Iowa State Coll. of Agric. and Mech. Arts, Ames, Iowa. 2323 Donald St.

Holland, Sister M. Charlotte. Registrar, St. Francis Xavier Coll. for Women, Chicago 15, I11.

Hollcroft, Mary P. (Mrs. T. R.). Wells Coll., Aurora, N.Y.

Hollcroft, Temple. 4529 Walsh St., Chevy Chase, Md.

Hollcroft, T. R. Prof., Wells Coll., Aurora, N.Y.

Holley, Dr. J. L. Economic Analyst, Treasury Dept., Washington 25, D.C. 2700 Q St. N.W., Washington 7. 
Holmes, C. T. Prof., Bowdoin Coll., Brunswick, Me. 60 Spring St.

Holtom, Carl. Asso. Prof., Air Forces Inst. of Tech., Wright Field, Ohio.

Hood, R. T. Asst., Univ. of Wisconsin, Madison 6, Wis. 705 Langdon St., Madison 5 .

Hopf, Heinz. Prof., Swiss Federal School of Tech., Zürich, Switzerland.

Hopkins, L. A. Dir., Summer Session, and Prof., Univ. of Michigan, Ann Arbor, Mich.

Hopkins, Dr. Margarete W. (Mrs. E. J.). 196 Clinton Ave., Brooklyn 5, N.Y.

Horn, Alfred. Asst. Prof., Univ. of California at Los Angeles, Los Angeles 24, Calif.

Horsfall, I. O. Dir., Extension Div., and Prof., Univ. of Utah, Salt Lake City 1, Utah.

Horton, R. E. Instr., Los Angeles City Coll., Los Angeles 27, Calif. 4655 Los Feliz Blvd.

Hosier, W. A. Actuarial Asst., Monarch Life Ins. Co., 365 State St., Springfield, Mass. 274 Forest Park Ave.

Hoskins, R. H. Actuarial Clerk, John Hancock Mutual Life Ins. Co., 197 Clarendon St., Boston 16, Mass.

Hostetter, I. M. Asso. Prof., Oregon State Coll., Corvallis, Ore.

Hostinsky, L. Aileen. Asst., Univ. of Illinois, Urbana, Ill. 153 Mathematics Bldg.

Hotelling, Harold. Asso. Dir. and Prof., Inst. of Stat., Univ. of North Carolina, Chapel Hill, N.C. P.O. Box 168.

Houghton, D. B. Research Eng., Franklin Inst., Philadelphia 3, Pa.

Househam, K. O. Princeton Univ., Princeton, N.J. 101 Broadmead.

Householder, Dr. A. S. Chief Physicist, Oak Ridge National Lab., Oak Ridge, Tenn.

Houston, W. V. Pres. and Prof. of Physics, Rice Inst., Houston 1, Tex. Box 1892.

Hove, E. Marie. Instr., Hofstra Coll, Hempstead, N.Y.

Hovey, B. K. Asso. Prof., Elec. Eng. Dept., Univ. of Pittsburgh, Pittsburgh 13, Pa. 208 Thaw Hall.

Howard, Aughtum S. (Mrs. N. J.). Prof., Kentucky Wesleyan Coll., Winchester, $\mathrm{Ky}$.

Howard, B. E. Asst., Univ. of Illinois, Urbana, Ill.

Howell, A. C. John Hancock Mutual Life Ins. Co., 197 Clarendon St., Boston 17, Mass. 167 Beacon St., Boston 16.

Howell, J. L. Asst., Yale Univ., New Haven 11, Conn.

Howland, L. A. Dean Emeritus and Prof. Emeritus, Wesleyan Univ., Middletown, Conn. 29 Gordon P1.

Hoy, E. A. Chief, Research and Evaluation Sec., Naval Reserve Training Publications Project, Naval Gun Factory, Washington 25, D.C. 2800 Erie St. S.E., Washington 20.

Hoyt, R. S. Retired. 827 Summit Ave., River Edge, N.J.

Hratz, Rev. J. A. Asst. Prof., St. Ambrose Coll., Davenport, Iowa.

Hsieh, L. F. Instr., Shantung Univ., Tsingtao, Shantung, China.

Hsiung, Dr. C. C. Instr., Univ. of Wisconsin, Madison 6, Wis.

Hsti, H. T. Yale Univ., New Haven 11, Conn. 2678 Yale Station.

Hsu, P. L. Asso. Prof., Inst. of Stat., Univ. of North Carolina, Chapel Hill, N.C. Box 168.

Hua, L. K. Visiting Prof., Univ. of Illinois, Urbana, Ill.

Hubbs, H. N. Sec., Treas., and Prof., Hobart and William Smith Colls., Geneva, N.Y.

Hubert, W. G. Asso. Prof., City Coll., New York 31, N.Y.

Huck, Raymond. Instr., Johns Hopkins Univ., Baltimore 18, Md. Rowland Hall.

Huff, G. B. Prof., Univ. of Georgia, Athens, Ga. Fellow, Harvard Univ., Cambridge 38, Mass. 30 Gould Rd., Arlington 74, Mass.

Huff, W. N. Asst. Prof., Univ. of Oklahoma, Norman, Okla.

Huffer, R. C. Prof., Beloit Coll., Beloit, Wis.

Hughart, S. P. Instr., Univ. of Chicago, Chicago 37, I1l. 411 Eckhart Hall.

Hughes, H. K. Prof., Purdue Univ., Lafayette, Ind.

Hull, Evelyn M. Office Manager, American Math. Society, 531 W. 116th St., New York 27, N.Y. 
Hull, Ralph. Prof., Purdue Univ., Lafayette, Ind.

Humm, Dr. D. G. Dir., Humm Personnel Service, 1219 W. 12th St., Los Angeles 15, Calif. P.O. Box 1433, Del Valle Station.

Hummel, P. M. Prof., Univ. of Alabama, University, Ala. Box 1251.

Humphreys, M. Gweneth. Asst. Prof., Newcomb Memorial Coll., Tulane Univ. of Louisiana, New Orleans 18, La.

Humphreys, T. R. Asst. Prof., New Jersey State Teachers Coll., Upper Montclair, N.J. 118 Union St., Montclair, N.J.

Humphreys, W. J. Prof. Emeritus, George Washington Univ., Washington 6, D.C. Cosmos Club, Washington 5.

Hunsaker, N. C. Asso. Prof., Utah State Agric. Coll., Logan, Utah.

Hunt, G. H. Asst. Prof. Emeritus, Univ. of California at Los Angeles, Los Angeles 24, Calif. 237 Tavistock Ave.

Hunt, Mildred. Registrar and Prof., Illinois Wesleyan Univ., Bloomington, Ill.

Hunter, Louise S. Asso. Prof., Virginia State Coll., Petersburg, Va.

Hunter, Dr. R. G. Retired. 525 Country Club Blvd., Des Moines 12, Iowa.

† Huntington, E. V. Prof. Emeritus, Harvard Univ., Cambridge 38, Mass. 48 Highland St.

Huntley, H. B. Math., Cox and Stevens Aircraft Corp., Box 30, Mineola, N.Y.

Hurd, Dr. C. C. Technical Research Head, Carbide and Carbon Chemicals Corps., Oak Ridge, Tenn.

Hurewicz, Witold. Asso. Prof., Massachusetts Inst. of Tech., Cambridge 39, Mass.

Hurst, J. W. Prof., Montana State Coll., Bozeman, Mont. 522 S. 6th St.

Hurt, J. M. Instr., Univ. of Texas, Austin 12, Tex. 408A W. 21st St., Austin 21.

Hurt, J. T. Prof., Agric. and Mech. Coll. of Texas, College Station, Tex.

Hurwitz, Dr. Solomon. Instr., City Coll., New York 31, N.Y. 4014 Ave. I, Brooklyn 10, N.Y.

† Hurwitz, W. A. Prof., Cornell Univ., Ithaca, N.Y. White Hall.

Huskey, Dr. H. D. Math., Inst. for Numerical Analysis, National Bur. of Standards, Univ. of California at Los Angeles, Los Angeles 24, Calif.

Huston, R. E. Prof., Rensselaer Polytech. Inst., Troy, N.Y. 6 Broadview Ter.

Hutcherson, W. R. Prof., Northwestern State Coll., Natchitoches, La.

Hutchings, w. L. Asso. Prof., Whitman Coll., Walla Walla, Wash.

Hutchinson, C. A. Prof., Coll. of Eng., Univ. of Colorado, Boulder, Colo.

Hutchinson, J. D. Asso. Prof., Univ. of Houston, Houston 4, Tex. 5415 Yupon, Bellaire, Tex.

Hutchinson, L. C. Asso. Prof., Polytech. Inst. of Brooklyn, Brooklyn 2, N.Y.

Hyden, J. A. Prof., Vanderbilt Univ., Nashville 4, Tenn. Box 111.

Hyers, D. H. Asso. Prof., Univ. of Southern California, Los Angeles 7, Calif.

Hyman, $\dot{M}$. A. Math., Naval Ordnance Lab., White Oak, Silver Spring 19, Md. 4000 Kansas Ave. N.W., Washington 11, D.C.

Ikenberry, Ernest. Instr., Louisiana State Univ. and Agric. and Mech. Coll., Baton Rouge 3, La. 228 Maximilian St., Baton Rouge 10.

Indritz, Jack. Instr., Univ. of Minnesota, Minneapolis 14, Minn. 119 Folwell Hall.

Ingersoll, B. M. 534 W. 124th St., New York 27, N.Y.

Ingham, A. E. Lect., King's Coll., Cambridge Unniv., Cambridge, England.

Ingraham, M. H. Dean and Prof., Univ. of Wisconsin, Madison 6, Wis. South Hall.

Ingram, Rev. R. E. Lect., Univ. of Ireland, Dublin, Ireland. Rathfornham Castle. Ingram, W. H. 150 Claremont Ave., New York 27, N.Y.

Irick, P. E. Instr., Purdue Univ., Lafayette, Ind.

Irwin, Frank. Asso. Prof. Emeritus, Univ. of California, Berkeley 4, Calif. 2921 Regent St., Berkeley 5.

Irwin, P. C. Actuarial Vice Pres., Equitable Life Ins. Co. of Iowa, Des Moines 6, Iowa.

Isaacs, Rufus. Research Eng., North American Aviation, Inc., Municipal Airport, Los Angeles 45, Calif. 8449 Truxton Ave.

Isaacson, Eugene. Instr., Inst. for Math. and Mech., New York Univ., New York 3, N.Y. 175-27 Wexford Ter., Jamaica 3, N.Y. 
Isaacson, S. L. Columbia Univ., New York 27, N.Y. 2523 Loyola Southway, Baltimore 15, Md.

Isenberg, J. S. Research Asso., Grad. Div. of Applied Math., Brown Univ., Providence 12, R.I.

Ito, W. H. Instr., Univ. of Minnesota, Minneapolis 14, Minn. 204 Main Engineering.

Iverson, P. F. Prof., Potomac State School, West Virginia Univ., Keyser, W.Va.

Iwanchuk, R. Y. Asst. Prof., Kent State Univ., Kent, Ohio.

Iwanowski, R. M. Instr., Sweet Briar Coll., Sweet Briar, Va.

Jablon, Seymour. 200 W. 108th St., New York 25, N.Y.

Jabotinsky, E. T. Publisher, Riveon Lematematika, 39 Gaza Rd., Jerusalem. 30 W. 90th St., New York 24, N.Y.

Jacka, R. C. Lect., Univ. of Alberta, Edmonton, Alta., Canada.

Jackson, J. B. Dean of Men, Univ. of South Carolina, Columbia 19, S.C. 227 S. Waccamaw Ave., Columbia 48.

Jackson, L. L. Retired. Box 688, R.F.D. 6, Tucson, Ariz.

Jackson, Rosa L. Prof., Alabama Coll., Montevallo, Ala. 211 Moody St.

Jackson, S. B. Prof., Univ. of Maryland, College Park, Md.

Jacobson, A. W. Asst. Prof., Wayne Univ., Detroit 1, Mich. 18735 Greenview, Detroit 19.

Jacobson, Florence D. (Mrs. Nathan). 12 Wadsworth St., Hamden, Conn.

Jacobson, Nathan. Asso. Prof., Yale Univ., New Haven 11, Conn. Leet Oliver Memorial Hall.

Jacobus, Dr. D. S. Retired. 93 Harrison Ave., Montclair, N.J.

Jacoby, A. R. Asst. Prof., Univ. of Miami, Coral Gables 34, Fla.

Jaeger, C. G. Prof., Pomona Coll., Claremont, Calif.

Jaeger, Dr. J. C. Lect., Univ. of Tasmania, Hobart, Tasmania, Australia.

Jaffe, Haym. Asso. Prof., Pennsylvania Military Coll., Chester, Pa. 248 Rocklyn Rd., Upper Darby, Pa.

Jaffe, W., J. Asst. Prof., Industrial Eng. Dept., Newark Coll. of Eng., Newark 2, N.J. 1030 Anderson Ave., Palisade, N.J.

Jaffee, W. A. Math., Univ. of Chicago, Chicago 37, Ill. 1441 Albion Ave., Chicago 26.

James, Glenn. Asso. Prof., Univ. of California at Los Angeles, Los Angeles 24, Calif.

James, Dr. R. C. Instr., Univ. of California, Berkeley 4, Calif.

James, R. D. Prof., Univ. of British Columbia, Vancouver, B.C., Canada.

Jamrich, J. X. Asst., Univ. of Wisconsin, Madison 6, Wis.

Janes, w. C. Asso. Prof., Kansas State Coll. of Agric. and Applied Science, Manhattan, Kan.

Janko, J. J. Prof., Univ. of Tech. Sciences, Prague II, Czechoslovakia.

Janssen, R. E. Asst., Univ. of Illinois, Urbana, I11. 153 Mathematics B1dg.

Jaramillo, Dr. T. J. Research Math., Armour Research Foundation, Illinois Inst. of Tech., Chicago 16, I11. 1947 S. Kedzie Ave., Chicago 23.

Jardetzky, Wenceslas. Visiting Prof., Univ. of Graz, Graz, Austria. 120 Hilmteich St., Graz-Kroisbach, Austria.

Jarnagin, M. P., Jr. 630 Milledge Circle, Athens, Ga.

Jastrow, Robert. Lect., Physics Dept., Columbia Univ., New York 27, N.Y. Pupin Physics Labs.

Jeeves, T. A. Lect. and Research Asst., Stat. Lab., Univ. of California, Berkeley 4, Calif. 2511 Hearst Ave., Berkeley 9.

Jeffery, R. L. Prof., Queen's Univ., Kingston, Ont., Canada.

Jeffrey, Charlotte D. (Mrs. T. E.). Psychometrician, Psychometric Lab., Univ. of Chicago, Chicago 37, Ill. 9857 Hoxie Ave., Chicago 17.

Jeffries, J. B. Prof., Physics Dept., Agric. and Tech. Coll. of North Carolina, Greensboro, N.C. 923 Sevier St.

Jeffries, Marion B. Univ. of Virginia, Charlottesville, Va. 507 Westview Ave., Bedford, Va. 
Jehle, Herbert. Asst. Prof., Physics Dept., Univ. of Pennsylvania, Philadelphia 4, $\mathrm{Pa}$. Randal Morgan Lab. of Physics.

Jenkins, E. D. Asso. Prof., Kent State Univ., Kent, Ohio.

Jenkins, Dr. J. A. Fellow, Harvard Univ., Cambridge 38, Mass. 87 Belsize Dr., Toronto 12, Ont., Canada.

Jennings, S. A. Prof., Univ. of British Columbia, Vancouver, B.C., Canada.

Jennings, Walter. Asst. Prof., U. S. Naval Postgrad. School, Annapolis, Md.

Jensen, Dr. C. M. Lect., Univ. of Minnesota, Minneapolis 14, Minn. 3315 17th Ave. S., Minneapolis 7.

Jerbert, A. R. Asso. Prof., Univ. of Washington, Seattle 5, Wash. 5033 Pullman Ave.

Jerison, Meyer. Univ. of Michigan, Ann Arbor, Mich. 1630 Tully Ct., Willow Run Village, Mich.

Jessen, Børge. Prof., Univ. of Copenhagen, Copenhagen $\varnothing$, Denmark. Maltegårdsvej 11, Gentofte, Denmark.

†Joffe, S. A. Retired. 515 W. 110th St., New York 25, N.Y.

Johanson, R. N. Asso. Prof., Boston Univ., Boston 16, Mass.

John, Fritz. Asso. Prof., New York Univ., New York, N.Y. 30 Summit Ave., New Rochelle, N.Y.

Johns, A. E. Prof., McMaster Univ., Hamilton, Ont., Canada.

Johnsen, Dr. Madeline M. Instr., Purdue Univ., Lafayette, Ind.

Johnson, Evan. Prof., Pennsylvania State Coll., State College, Pa. P.O. Box 321.

Johnson, P. B. Asst. Prof., Occidental Coll., Los Angeles 41, Calif.

Johnson, R. A. Prof., Brooklyn Coll., Brooklyn 10, N.Y. 3315 80th St., Flushing, N.Y.

Johnson, R. B. Teaching Asst., Univ. of Tennessee, Knoxville 16, Tenn. 907 Mountcastle St.

Johnson, R. E. Asso. Prof., Smith Coll., Northampton, Mass. Lect., Yale Univ., New Haven 11, Conn.

Johnson, Roberta F. Asso. Prof., Wilson Coll., Chambersburg, Pa.

Johnson, S. M. Asst., Univ. of Illinois, Urbana, Ill. 408 S. Goodwin.

Johnston, F. E. Prof., George Washington Univ., Washington 6, D.C.

Jonah, H. F. S. Asso. Prof., Purdue Univ., Lafayette, Ind.

Jones, A. W. Asso. Prof., Rensselaer Polytech. Inst., Troy, N.Y. 352 Marshland Ct.

Jones, Bessie. Instr., Univ. of Alabama, University, Ala. 608 W. Adams St., Dothan, Ala.

Jones, B. W. Prof., Univ. of Colorado, Boulder, Colo.

Jones, C. D. Chief Eng., Meyer Eng. Co., Ferndale, Mich. 2500 Brockton W., Royal Oak, Mich.

Jones, F. B. Asso. Prof., Univ. of Texas, Austin 12, Tex. 1401 W. 29th St., Austin 21,

Jones, Dr. G. M. Architect and Eng., 437 E. Oakwood Blvd., Chicago 15, Ill.

Jones, H. T. Lehigh Univ., Bethlehem, Pa. $1032 \mathrm{Club}$ Ave., Allentown, Pa.

Jones, Dr. L. G. F. Staff Member, General Precision Lab., Inc., 63 Bedford Rd., Pleasantville, N.Y. 144 Columbus Ave., Valhalla, N.Y.

Jones, Margaret E. Asst. Prof., Ohio State Univ., Columbus 10, Ohio. 164 13th Ave., Columbus 1.

Jones, P. C. Science Editor, Bell Telephone Labs., Inc., 463 West St., New York 14, N.Y.

Jones, P. S. Asst. Prof., Univ. of Michigan, Ann Arbor, Mich.

Jonsson, Bjarni. Asst. Prof., Brown Univ., Providence 12, R.I.

Jordan, C. W. Asst. Prof., Williams Coll., Williamstown, Mass. Stetsam Rd.

Jordan, H. A. Asso. Prof., Colby Coll., Waterville, Me. P.O. Box 103.

Jordan, H. E. Asso. Prof. Emeritus, Univ. of Kansas, Lawrence, Kan. 1600 Kentucky St.

Jordan, Reba. Instr., Oklahoma Baptist Univ., Shawnee, Okla.

Joseph, Louis. Illinois Inst. of Tech., Chicago 16, Ill. 1306 S. Lawndale Ave., Chicago 23.

Julia, G. M. Prof., Univ. of Paris, Paris 5, France. 4b Traversiere St., Versailles, France. 
Juncosa, M. L. Instr., Johns Hopkins Univ., Baltimore 18, Md.

Justice, H. K. Asst. Dean and Prof., Coll. of Eng., Univ. of Cincinnati, Cincinnati 21, Ohio.

Kac, Mark. Prof., Cornell Univ., Ithaca, N.Y.

Kagno, Dr. I. N. 3326 Bainbridge Ave., New York 67, N.Y.

Kahal, Robert. Instr., Polytech. Inst. of Brooklyn, Brooklyn 2, N.Y.

Kahn, Herman. 330 $1 / 2$ N. Spalding Ave., Los Angeles 36, Calif.

Kaitz, H. B. Economic Analyst, Commerce Dept., Washington 25, D.C. 1919 19th St. N.W., Washington 9.

Kales, Dr. M. L. Radio Eng., Naval Research Lab., Washington 20, D.C. 4692 Nichols Ave. S.W.

Kalinowski, Rev. Walbert. Asst. Prof., St. John's Univ., Collegeville, Minn.

Kalisch, G. K. Asst. Prof., Univ. of Minnesota, Minneapolis 14, Minn.

Kalish, Aida. 420 Crown St., Brooklyn 25, N.Y.

Kamel, Hyman. Instr., Univ. of Pennsylvania, Philadelphia 4, Pa. 4107 Leidy Ave.

Kampé de Fériet, Joseph. Prof., Univ. of Lille, Lille, France.

Kanter, L. H. Asst. Prof., Univ. of Arkansas, Fayetteville, Ark.

Kantz, A. D. Asst. Prof., Southwestern Coll., Winfield, Kan.

Kaplan, E. L. Instr., Coll. of Eng. and Science, Carnegie Inst. of Tech., Pittsburgh $13, \mathrm{~Pa}$.

Kaplan, Samuel. Asst. Prof., Wayne Univ., Detroit 1, Mich. 2472 Glendale, Detroit 6.

Kaplan, Wilfred. Asst. Prof., Univ. of Michigan, Ann Arbor, Mich. 1308 Olivia Ave.

Kaplansky, Irving. Asst. Prof., Univ. of Chicago, Chicago 37, Ill. Member, Inst. for Advanced Study, Princeton, N.J.

Karlin, Dr. Meyer. Math., Computation Lab., National Bur. of Standards, 150 Nassau St., New York 7, N.Y. 1804 E. 4th St., Brooklyn 23, N.Y.

Karlin, Dr. Samuel. Instr., California Inst. of Tech., Pasadena 4, Calif.

Karnes, H. T. Asso. Prof., Louisiana State Univ. and Agric. and Mech. Coll., Baton Rouge 3, La.

Karp, Dr. S. N. Senior Research Scientist, Inst. for Math. and Mech., New York Univ., New York 3, N.Y.

Karush, William. Asst. Prof., Univ. of Chicago, Chicago 37, Ill. Eckhart Hall.

Kaskey, Gilbert. Instr., Univ. of Delaware, Newark, Del. 17 New St.

†Kasner, Edward. Prof., Columbia Univ., New York 27, N.Y. 430 W. 116th St.

Kasriel, R. H. Instr., Univ. of Virginia, Charlottesville, Va. 105 Observatory Ave.

Kato, Chosaburo. Asso. Prof., Denison Univ., Granville, Ohio. P.O. Box 105.

Katz, Leo. Asst. Prof., Michigan State Coll., East Lansing, Mich.

Katz, Stanley. Hydrocarbon Research, Inc., 115 Broadway, New York 6, N.Y.

Katz, William. Attorney, 70 Pine St., New York 5, N.Y. 1574 Crotona Park E., New York 60.

Kaufman, Bruria (Mrs. Z. S. Harris). 2222 N. 53d, Philadelphia 31, Pa.

Kavanagh, Dr. A. J. Senior Research Physicist, Scientific Instrument Div., American Optical Co., Buffalo 15, N.Y. Box A.

Kavanau, J. L. Fellow, Univ. of California at Los Angeles, Los Angeles 24, Calif. 2311 Malcolm Ave.

Kean, H. P. Asso. Prof., McMurry Coll., Abilene, Tex. 1234 Peach St.

Kearney, Dora E. Itasca Junior Coll., Coleraine, Minn.

Keck, Winfield. Fellow, Physics Dept., Brown Univ., Providence 12, R.I. 74 Benefit St., Providence 3.

Keeler, Dr. I. F. 219 S. Columbia, Naperville, I11.

Keffer, Ralph. Actuary, Aetna Life Ins. Co., Hartford 15, Conn.

Kellar, Margaret E. Editorial Asst., American Math. Society, 531 W. 116th St., New York 27, N.Y. 616 W. 116th St.

Keller, M. W. Asso. Prof., Purdue Univ., Lafayette, Ind.

Kelley, J. L. Asso. Prof., Univ. of California, Berkeley 4, Calif. 2327 Derby Ave., Berkeley 5. 
Kells, L. M. Prof., U.S. Naval Acad., Annapolis, Md. 23 Thompson St.

Kelly, J. B. Instr., Univ. of Wisconsin, Madison 6, Wis. 1521 Madison St., Madison 5 .

Kelly, L. M. Asst. Prof., Michigan State Coll., East Lansing, Mich.

Kelly, Dr. P. J. Instr., Univ. of Southern California, Los Angeles 7, Calif. 11773 Montana Ave., Los Angeles 24.

Kemble, E. C. Prof., Physics Dept., Harvard Univ., Cambridge 38, Mass.

Kempner, A. J. Prof., Univ. of Colorado, Boulder, Colo.

Kendall, Claribel. Prof., Univ. of Colorado, Boulder, Colo. 1305 Euclid Ave.

Kennedy, E. S. Adj. Prof., American Univ. of Beirut, Beirut, Lebanon.

Kenney, J. F. Asso. Prof., Univ. of Wisconsin in Milwaukee, Milwaukee 3, Wis. 623 W. State St.

Kennison, L. S. Asst. Prof., Brooklyn Coll., Brooklyn 10, N.Y. Lt. Com. U.S.N.R., Hq., 3d Naval District, 90 Church St., New York 7, N.Y. 110-11 215th St., Jamaica 9, N.Y.

Kent, J. R. F. Asso. Prof., Triple Cities Coll., Syracuse Univ., Endicott, N.Y.

Kernaghan, Mother Marie. Prof., Maryville Coll., St. Louis 18, Mo.

Kersten, H. J. Prof., Dept. of Biophysics, Univ. of Cincinnati, Cincinnati 21, Ohio.

Kester, W. W. Asst. Prof., Southwestern Univ., Georgetown, Tex.

Ketchum, Dr. Gertrude S. (Mrs. P.W.). 803 W. Illinois St., Urbana, Ill.

Ketchum, P. W. Prof., Univ. of Illinois, Urbana, Ill. 803 W. Illinois St.

Keyser, Sarah Y. (Mrs. C. J.). Dean, Nightingale-Bamford School, New York 28, N.Y. 50 Morningside Dr., New York 25.

Kibbey, D. E. Asso. Prof., Syracuse Univ., Syracuse 10, N.Y.

Kiernan, P. J. Instr., Lawrenceville School, Lawrenceville, N.J.

Kieval, Dr. H. S. Instr., Brooklyn Coll., Brooklyn 10, N.Y.

Kimball, Dr. B. F. Senior Stat., New York State Public Service Comm., 233 Broadway, New York 7, N.Y. 33 Bogart Ave., Port Washington, N.Y.

Kimball, S. H. Prof., Univ. of Maine, Orono, Me. 135 Stevens Hall.

Kincaid, Dr. W. M. Instr., Univ. of Michigan, Ann Arbor, Mich.

Kinerk, H. E. Designing Eng., Western Gear Works, Seattle, Wash. 528 22d Ave. N., Seattle 2.

King, A. L. Prof., Physics Dept., Dartmouth Coll., Hanover, N.H.

Kingsbury, R. S. Staff Member, Operations Evaluation Group, Div. of Industrial Cooperation, Massachusetts Inst. of Tech., Cambridge 39, Mass. OEG (CNO), Room 3827, Navy Dept., Washington 25, D.C.

Kingston, H. R. Dean, Coll. of Arts and Science, Univ, of Western Ontario, London, Ont., Canada.

Kingston, J. M. Asst. Prof., Univ. of Washington, Seattle 5, Wash.

Kinney, J. M. Prof. Emeritus, Wilson Junior Coll., Chicago 21, Ill. 8058 Bennett Ave., Chicago 17.

Kinney, J.' R., Asst., Univ. of Illinois, Urbana, I1l. 160 Mathematics Bldg.

Kiokemeister, Fred. Asso. Prof., Mt. Holyoke Coll., South Hadley, Mass.

Kirby, A. R. Asst. Prof., School of Education, Fordham Univ., New York 58, N.Y. 2921 Briggs Ave.

Kirby, Mary D. (Mrs. A. R.). Instr., Manhattanville Coll. of the Sacred Heart, New York 27, N.Y. 2921 Briggs Ave., New York 58.

Kirk, D. B. Actuarial Student, Mutual Benefit Life Ins. Co., Box 359, Newark 1, N.J. 5131 Spruce St., Philadelphia 39, $\mathrm{Pa}$.

Kiss, S. A. Patent Attorney, Standard Oil Development Co., 30 Rockefeller Plaza, New York 21, N.Y. 11 E. 92d St., New York 28.

Kitchens, J. W. Scientist, Naval Observ., Washington 25, D.C.

Kjosness, O. C. Dean of Admin. and Prof., Western State Coll., Gunnison, Colo.

Klamkin, M. S. Instr., Polytech. Inst. of Brooklyn, Brooklyn 2, N.Y.

Klauber, L. M. Pres., San Diego Gas and Elec. Co., San Diego, Calif. 233 W. Juniper St., San Diego 1.

Klee, V. L. Fellow, Univ. of Virginia, Charlottesville, Va. Montibello Circle.

Kleene, S. C. Prof., Univ. of Wisconsin, Madison 6, Wis.

Klimczak, Dr. W. J. Instr., Univ. of Rochester, Rochester 3, N.Y.

Kline, J. R. Prof., Univ. of Pennsylvania, Philadelphia 4, Pa. 529 Riverview Rd., Swarthmore, $\mathrm{Pa}$. 
Kline, Morris. Asso. Prof., New York Univ., New York 3, N.Y. 1188 E. 8th St., Brooklyn 30, N.Y.

Kline, Vivian W. (Mrs. A. P.). Asst. Prof., Northeast Missouri State Teachers Coll., Kirksville, Mo. 601 E. McPherson St.

Klipple, E. C. Prof., Agric. and Mech. Coll. of Texas, College Station, Tex. P.O. Box 4852 .

Kloosterman, H. D. Prof., Univ. of Leiden, Leiden, Netherlands. Van Oldenbarneveltstraat 52 .

Kloss, R. E. Weather Observer, U.S. Weather Bur., Raton, N.M. 238 Park Ave.

Kneale, S. G. Instr., Univ. of Kansas, Lawrence, Kan.

Knebelman, M. S. Prof., State Coll. of Washington, Pullman, Wash. 2008 Indiana St.

Knechtel, B. G. Instr., Aurora Coll., Aurora, Ill.

Knedler, P. A. Prof., State Teachers Coll., Kutztown, Pa. East Texas, Pa.

Knifley, Louise M. Instr., Marshall Coll., Huntington, W.Va.

Knight, K. V. Physicist, Bur. of Ordnance, Navy Dept., Washington 25, D.C. 271931 st St. S.E., Washington 20.

Knight, L. C. Asst. Prof., Muskingum Coll., New Concord, Ohio. 156 Lakeside.

Knobelauch, Dr. E. A. Research Asso., Moore School of Elec. Eng., Univ. of Pennsylvania, Philadelphia 4, Pa. Kellers Church, $\mathrm{Pa}$.

Knowler, L. A. Prof., State Univ. of Iowa, Iowa City, Iowa.

Knox, R. H. Asso. Prof., Virginia Military Inst., Lexington, Va. 313 Letcher Ave.

Kober, Hermann. 91 Weoley Park Rd., Selly Oak, Birmingham 29, England.

Koehler, Rev. C. F. Woodstock Coll., Woodstock, Md.

Koehler, Fulton. Asso. Prof., Univ. of Minnesota, Minneapolis 14, Minn. 50 Melbourne Ave. S.E.

Koehler, T. L. Asst. Prof., Muhlenberg Coll., Allentown, Pa. 625 N. 24th St.

Kogbetliantz, E. G. Prof., New School for Social Research, New York 11, N.Y. 438 W. 116th St., New York 27.

Koken, J. C. Instr., Univ. of Missouri, Columbia, Mo. 1318 Ross St.

Kokomoor, F. W."Prof., Univ. of Florida, Gainesville, Fla. Peabody 106.

Kolchin, E. R. Asst. Prof., Columbia Univ., New York 27, N.Y. Hamilton Hall.

Komm, Horace. Asst. Prof., Univ. of Rochester, Rochester 7, N.Y.

Konijn, H. S. 2339 Blake St., Berkeley 4, Calif.

Koopman, B. O. Prof., Columbia Univ., New York 27, N.Y.

Kopp, Paul. Asst., Patent Dept., Gulf Oil Corp., 1240 Munsey Bldg., Washington 4, D.C. 1305 N. Adams St., Arlington, Va.

Kormes, Dr. Jennie P. (Mrs. Mark). Instr., Brooklyn Coll., Brooklyn 10, N.Y. 119 E. 84th St., New York 28, N.Y.

Kormes, Dr. Mark. Consulting Actuary, 341 Madison Ave., New York 17, N.Y. 119 E. 84th St., New York 28.

Korzybski, Alfred. Dir., Inst. of General Semantics, Lakeville, Conn.

Kosambi, D. D. Prof., Tata Inst. of Fundamental Research, 53 Pedder Rd., Bombay 26, India.

Koss, W. E. Asst. Prof., Agric. and Mech. Coll. of Texas, College Station, Tex.

Kossack, C. F., Asso. Prof., Purdue Univ., Lafayette, Ind.

Kossack, C. R. Asst. Prof., State Teachers Coll., New Haven, Conn. 128 Bayard Ave., Hamden 14, Conn.

Kovarik, A. F. Prof. Emeritus, Yale Univ., New Haven 11, Conn. 145 Cottage St.

Kozakiewicz, Waclaw. Asso. Prof., Univ. of Saskatchewan, Saskatoon, Sask., Canada.

Krabill, D. M. Asso. Prof., Bowling Green State Univ., Bowling Green, Ohio.

Kraft, Ona. Teacher, Collinwood High School, Cleveland, Ohio. 11328 Euclid Ave., Cleveland 6.

Krall, H. L. Prof., Pennsylvania State Coll., State College, Pa.

Kramer, M. S. Instr., Univ. of Illinois, Urbana, Ill. 114 W. Clark St., Champaign, Ill.

Kramer-Lassar, Dr. Edna E. (Mrs. B. T. Lassar). Teacher, Thomas Jefferson High School, Brooklyn, N.Y. 32 Lenox Rd., Brooklyn 26.

Krasner, Dr. Marc. Chief of Research, National Center of Scientific Research, Paris 7, France. 101 Mont-Cenis St., Paris 18. 
Krathwohl, W. C. Prof. and Dir. of Tests, Inst. for Psychological Services, Illinois Inst. of Tech., Chicago 3, I11.

Kratz, P. H. Research Admin., Navy Dept., Washington, D.C. 1623 W. Westmoreland St., Philadelphia 40, Pa.

Kravetz, Saul. 3072 Brighton 13th St., Brooklyn 24, N.Y.

Kravitz, Sidney. Math., Ballistic Research Labs., Aberdeen Proving Ground, Md.

Kravtchenko, Julien. Prof., Univ. of Grenoble, Grenoble, France. 1 Perigord St., Toulouse, France.

Kreezer, G. L. Asso. Prof., Psychology Dept., Washington Univ., St. Louis 5, Mo.

Kremer, Rev. B. P. Instr., John Carroll Univ., Cleveland 18, Ohio.

Krieger, Cecilia. Asst. Prof., Univ. of Toronto, Toronto 5, Ont., Canada. Visiting Lect., Univ. of British Columbia, Vancouver, B.C., Canada.

Krug, R. E. Lib., Milwaukee Public Lib., 814 W. Wisconsin Ave., Milwaukee 3, Wis.

Krumhansl, J. A. Asst. Prof., Physics Dept., Brown Univ., Providence 12, R.I.

Kruse, W. E. Instr., St. Peter's Coll., Jersey City 6, N.J. 320 Webster Ave., Jersey City 7.

Krzywoblocki, M. Z. Asso. Prof., Aeronautical Eng. Dept., Univ. of Illinois, Urbana, Ill.; Consultant, Theoretical Mech. Subdiv., Naval Ordnance Lab., White Oak, Silver Spring 19, Md.

Kubis, J. F. Asso. Prof., Psychology Dept., Fordham Univ., New York 58, N.Y.

Kuebler, R. R. Asso. Prof., Dickinson Coll., Carlisle, Pa.

Kuhn, H. W. Prof. Emeritus, Ohio State Univ., Columbus 10, Ohio. 1179 Fairview Ave., Columbus 12.

Kuiper, Dr. N. H. Member, Inst. for Advanced Study, Princeton, N.J.

van der Kulk, Wouter. Asst. Prof., Brown Univ., Providence 12, R.I.

Kuller, R. G. Univ. of Michigan, Ann Arbor, Mich. 1931 Park Ave., Baltimore $17, \mathrm{Md}$.

Kuo, Dr. Y. H. Instr., Grad. School of Aeronautical Eng., Cornell Univ., Ithaca, N.Y.

† Kuschke, Dr. C. G. P. Retired. 1662 Shatto St., Los Angeles 14, Calif.

Kutman, Helen K. (Mrs.). Instr., Hunter Coll., New York 21, N.Y. 2825 Grand Concourse, New York 58.

L'Abbé, Maurice. Asst. Lect., Univ. of Montreal, Montreal 26, Que., Canada.

Lackay, Sister M. Pachomia. Instr., Coll. of St. Teresa, Kansas City 2, Mo.

Lachenbruch, S. H. Math. Physicist, National Bur. of Standards, Commerce Dept., Washington 25, D.C. 204 Elmira St. S.W., Washington 20.

Laden, Dr. H. N. Lt., U.S.N., U.S. Naval Postgrad. School, Annapolis, Md.

Laderman, Jack. Math., Computation Lab., National Bur. of Standards, 150 Nassau St., New York 7, N.Y. 1705 Andrews Ave., New York 53.

Laderman, Leonore M. (Mrs. P.). Tutor, Queens Coll., Flushing, N.Y. 115 Central Park W., New York 23, N.Y.

LaFon, Gladys Q. (Mrs. J. E.). 806 Monnett Ave., Norman, Okla.

Lagerstrom, P. A. Asst. Prof., Aeronautics Dept., California Inst. of Tech., Pasadena 4, Calif.

Laguardia, Rafael. Prof., Univ. of Uruguay, Montevideo, Uruguay. 1329 Mercedes.

Lambert, W. D. Retired. Box 687, Canaan, Conn.

Lampland, Dr. C. O. Astronomer, Lowell Observ., Flagstaff, Ariz. Box 1640.

Lamson, K. W. Asso. Prof., Lehigh Univ., Bethlehem, Pa.

Lancaster, Dr. O. E. Math., Bur. of Aeronautics, Navy Dept., Washington 25, D.C. 460727 th St., Mt. Rainier, Md.

Lanckton, A. L. Manager, Socony-Vacuum Oil Co. Box 163, Athens, Greece.

Lanczos, Dr. Cornelius. Research Eng., Boeing Aircraft Co., Seattle 14, Wash. 1916 Franklin P1., Seattle 2.

Landau, Dr. H. G. Math., Ballistic Research Labs., Aberdeen Proving Ground, Md. 259 Wilson St., Havre de Grace, Md.

Landers, A. W. Asst. Prof., Brooklyn Coll., Brooklyn 10, N.Y. 108-48 67th Dr., Forest Hills, N.Y. 
Landers, Mary K. (Mrs. A. W.). Asst. Prof., Hunter Coll., New York 21, N.Y. 108-48 67th Dr., Flushing, N.Y.

Landin, Joseph. Asst. Prof., Univ. of Illinois, Urbana, Ill.

Landry, A. E. Prof., Catholic Univ. of America, Washington 17, D.C. 3624 13th St. N.E.

Lane, E. P. Prof., Univ. of Chicago, Chicago 37, I1l. Eckhart Hall.

Lane, R. E. Asst. Prof., Univ. of Texas, Austin 12, Tex. 2807 Breeze Ter., Austin.

Lang, G. B. Asst. Prof., Univ. of Florida, Gainesville, Fla.

Lang, Kermit. Asst. Actuary, Equitable Life Ins. Co. of Iowa, Des Moines 6, Iowa.

Langebartel, R. G. Asst., Univ. of Illinois, Urbana, I11. 160 Mathematics Bldg.

Langenhop, C. E. Research Asst., Princeton Univ., Princeton, N.J. Fine Hall.

Langer, R. E. Prof., Univ. of Wisconsin, Madison 6, Wis. 822 Miami Pass., Madison 5.

Laning, Dr. J. H. Staff Member, Massachusetts Inst. of Tech., Cambridge 39, Mass. 39 Grant Ave., Watertown 72, Mass.

LaPaz, Lincoln. Prof., Univ. of New Mexico, Albuquerque, N.M.

Lapidus, Benjamin. Fellow, Univ. of Toronto, Toronto 5, Ont., Canada. 29 Howland Ave.

Lapidus, Leo. Instr., Michigan State Coll., East Lansing, Mich.

Larew, Gillie A. Prof., Randolph-Macon Woman's Coll., Lynchburg, Va.

Larguier, E. H. Prof., Spring Hill Coll., Spring Hill, Ala.

Larkin, Sister Francis X. Prof., St. Joseph's Coll. for Women, Brooklyn 5, N.Y.

Larrivee, J. A. Asst. Prof., Univ. of Vermont and State Agric. Coll., Burlington, $\mathrm{Vt}$.

Larsen, H. D. Prof., Albion Coll., Albion, Mich. 310 Burr Oak St.

Larsen, Thorstein. Asst. Prof., Elec. Eng. Dept., Johns Hopkins Univ., Baltimore $18, \mathrm{Md}$.

Larson. C. W. Asst., Univ. of Wisconsin, Madison 6, Wis. 1015 Clymer P1., Madison 5.

Larus, J. R. Vice Pres. and Actuary, Phoenix Mutual Life Ins. Co., Hartford 15 , Conn.

LaSala, Lucy. Teacher. 256 Irving Ave., Brooklyn 27, N.Y.

LaSalle, J. P. Asst. Prof., Univ. of Notre Dame, Notre Dame, Ind.

LaSalle, Margaret M. Asst., Louisiana State Univ. and Agric. and Mech. Coll., Baton Rouge, La. P.O. Box 7537, University Station.

Laserna, Mario. 6-35 19th St., Bogota, Colombia.

Lasley, J. W. Prof., Univ. of North Carolina, Chapel Hill, N.C. 523 E. Rosemary Lane.

Lassen, L. L. Asso. Prof., North Texas Agric. Coll., Agric. and Mech. Coll. of Texas, Arlington, Tex.

Latimer, C. G. Prof., Emory Univ., Emory University, Ga.

Latshaw, V. V. Asso. Prof., Lehigh Univ., Bethlehem, Pa. 708 8th Ave.

Latta, G. E. Lect., California Inst. of Tech., Pasadena 4, Calif.

Latter, Richard. California Inst. of Tech., Pasadena 4, Calif. 2050 Emperor Ave., Temple City, Calif.

Lauritzen, Dr. S. E. Lect., Tech. Univ. of Denmark, Copenhagen K, Denmark. Gentoftegade 51, Gentofte, Denmark.

Laush, George. Teaching Fellow, Cornell Univ., Ithaca, N.Y.

Lawler, Jane M. Project Eng. and Math., Aircraft Projects Sec., Armitage Field, Naval Ordnance Test Station, China Lake, Calif.

Lawrence, V. S. Asso. Prof., Virginia Polytech. Inst., Blacksburg, Va.

Lawson, J. W. Asst. Prof., Univ. of Manitoba, Winnipeg, Man., Canada.

Lax, Anneli L. (Mrs. P. D.). Asst. Research Math., Inst. for Math. and Mech., New York Univ., New York 3, N.Y. 100 W. 61 st St., New York 23.

Lax, P. D. Inst. for Math. and Mech., New York Univ., New York 3, N.Y. 100 W. 61 st St., New York 23.

Layton, Lamar. Asst., Univ. of Illinois, Urbana, I11. 250 Mathematics Bldg.

Lazar, Dr. Nathan. Instr., Teachers Coll., Columbia Univ., New York 27, N.Y. 1728 E. 17th St., Brooklyn 29, N.Y. 
Leach, C. E. R.F.D. 2, Marion, Ohio. Private, U.S.A.

Leamon, W. V. Eng., Kellex Corp., 233 Broadway, New York 7, N.Y. Box 22, Cathedral Station, New York 25.

Learson, R. J. Vice Pres. and Actuary, Western and Southern Life Ins. Co., 400 Broadway, Cincinnati 2, Ohio.

Leavens, D. H. Retired. 1632 Wood Ave., Colorado Springs, Colo.

Leavitt, W. G. Asst. Prof., Univ. of Nebraska, Lincoln 8, Neb. 1535 Nemaha, Lincoln 2.

Lee, H. L. Asst. Prof., Univ. of Tennessee, Knoxville 16, Tenn. 132 Woodburn Dr.

Lee, J. R. Asst. to the Dean, Yale Univ., New Haven 11, Conn. 2785 Yale Station.

Lee, Mary A. Asst. Prof., Sweet Briar Coll., Sweet Briar, Va.

Lee, (T.) H. Adj. Prof., Univ. of South Carolina, Columbia 19, S.C.

Leech, J. S. Asst. Prof., Univ. of Chicago, Chicago 37, I11.

Lefschetz, Solomon. Prof., Princeton Univ., Princeton, N.J. Fine Hall.

Leger, G. F. Asst., Univ. of Illinois, Urbana, Ill.

Lehman, Margaret B. (Mrs. R. M.). Lect., Univ. of California at Los Angeles, Los Angeles 24, Calif. 2315 24th St., Santa Monica, Calif.

Lehmann, C. H. Instr., Cooper Union, New York 3, N.Y. 144-17 29th Ave., Flushing, N.Y.

Lehmann, E. L. Asst. Prof., Univ. of California, Berkeley 4, Calif. 40 Oakridge Rd., Berkeley 5.

Lehmer, D. H. Prof., Univ. of California, Berkeley 4, Calif. 942 Hilldale Ave., Berkeley 8.

Lehner, Dr. Joseph. Math., Hydrocarbon Research, Inc., 115 Broadway, New York 6, N.Y.

Lehr, Marguerite. Asso. Prof., Bryn Mawr Coll., Bryn Mawr, Pa. Cartref.

Lehrer, T. A. Columbia Univ., New York 27, N.Y. 940 Park Ave., New York 28.

Leibler, Dr. R. A. Math., Navy Dept. Washington 25, D.C. 3801 Nebraska Ave. N.W.

Leifer, H. R. Asst. Chief, Registration and Research Sec., Veterans Admin., Pittsburgh, Pa. 1059 N. Negley Ave., Pittsburgh 6.

Leight, W. G. Meteorologist, Extended Forecast Sec., Weather Bur., Washington 25 , D.C. 1362 Parkwood P1. N.W., Washington 10.

Leighton, Walter. Prof., Washington Univ., St. Louis 5, Mo. Box 146.

Leipnik, R. B. Fellow, Inst. for Advanced Study, Princeton, N.J. 222 Ewing St.

Leisenring, Dr. K. B. 521 E. Jefferson St., Ann Arbor, Mich.

LeJunter, J. J. Instr., Michigan State Coll., East Lansing, Mich.

LeLeiko, Max. Instr., Rutgers Univ., New Brunswick, N.J. 204 Engineering Bldg.

Lemme, M. M. Asso. Prof., San Diego State Coll., San Diego 5, Calif.

Lemmer, Rev. J. G. Asst. Prof., Philosophy Dept., West Baden Coll., Loyola Univ., West Baden Springs, Ind.

Lence, R. H. Instr., Northwestern Univ., Evanston, Ill.

Lengyel, Dr. B. A. Physicist, Naval Research Lab., Washington 20, D.C. 127 Joliet St. S.W.

Lennes, J. B. Instr., Univ. of Oklahoma, Norman, Okla. Faculty Exch.

† Lennes, N. J. Prof. Emeritus, Montana State Univ., Missoula, Mont. 1325 Gerald Ave.

Lenser, W. T. Instr., Univ. of Nebraska, Lincoln 8, Neb.

Lenzen, V. F. Prof., Physics Dept., Univ. of California, Berkeley 4, Calif.

Leonard, H. B. Prof., Univ. of Arizona, Tucson, Ariz. Box 4024, University Station.

Leone, F. C. Fellow, Purdue Univ., Lafayette, Ind.

Lepecki, Zbigniew. Visiting Prof., Univ. of Paraná, Paraná, Brazil. 47 Barao de Lucena St., Belo Horizonte, Minas Gerais, Brazil.

Lepson, Benjamin. Lect., Columbia Úniv., New York 27, N.Y.

Lesher, Marian A. Instr., Ft. Scott Junior Coll., Ft. Scott, Kan. 910 Crawford St.

Lester, Carolina A. Asst. Prof., New York State Coll. for Teachers, Albany 3, N.Y.

LeStourgeon, Flora E. Retired. 208 N.E. 5th Ct., Delray Beach, Fla. 
Leuschner, A. O. Prof. Emeritus, Univ. of California, Berkeley 4, Calif. 1816 Scenic Ave., Berkeley 9.

Leutert, W. W. Asst. Prof., Univ. of Maryland, College Park, Md.

Levenberg, Kenneth. Univ. of Maryland, College Park, Md. 6809 Dartmouth Ave.

Levene, Dr. Howard. Instr., Math. Stat. Dept., Columbia Univ., New York 27, N.Y. 22 E. 88th St., New York 28.

Levenson, Dr. M. E. Instr., School of Eng., Cooper Union, New York 3, N.Y.

LeVeque, Dr. W. J. Instr., Harvard Univ., Cambridge 38, Mass. 12 Hunt Hall.

Levi, Dr. Beppo. Dir., Inst. of Math., Univ. of Rosario, Rosario, Argentina. 2133 San Lorenzo.

Levi, F. W. Prof., Tata Inst. of Fundamental Research, 53 Pedder Rd., Bombay 26 , India.

Levi, Dr. Howard. Instr. Columbia Univ., New York 27, N.Y. 185 Claremont Ave.

Levin, Dr. J. H. Chief, Machines Branch, Computing Lab., Ballistic Research Labs., Aberdeen Proving Ground, Md.

Levin, Dr. Madeline. Instr., Hunter Coll., New York 21, N.Y. 542 W. 112th St., New York 25.

Levine, Jack. Prof., State Coll. of Agric. and Eng., Univ. of North Carolina, Raleigh, N.C. Box 5548, State College Station.

Levinson, Dr. H. C. Alewive Farm, Kennebunk, Me.

Levinson, Norman. Asso. Prof., Massachusetts Inst. of Tech., Cambridge 39, Mass. Mathematical Inst., Univ. of Copenhagen, Copenhagen, Denmark.

Levit, R. J. Asso. Prof., Univ. of Georgia, Athens, Ga.

Levitzki, Dr. Jakob. Lect., Hebrew Univ., Jerusalem.

Levy, Harry. Asso. Prof., Univ. of Illinois, Urbana, Ill.

Levy, Samuel. Physicist, National Bur. of Standards, Commerce Dept., Washington 25, D.C.

Levy, S. L. Instr., Illinois Inst. of Tech., Chicago 16, Ill. 3458 W. Polk St., Chicago 24.

Lew, E. A. Asst. Actuary, Metropolitan Life Ins. Co., 1 Madison Ave., New York 10, N.Y. 51 Mohegan Rd., Larchmont, N.Y.

Lewis, Anne L. Asst. Prof., Woman's Coll., Univ. of North Carolina, Greensboro, N.C.

Lewis, C. H. Instr., Orange Coast Coll., Costa Mesa, Calif. 343 S. Pickering Ave., Whittier, Calif.

Lewis, D. C. Prof., Johns Hopkins Univ., Baltimore 18, Md.

Lewis, D. J. Teaching Fellow, Univ. of Michigan, Ann Arbor, Mich.

Lewis, F. A. Prof., Univ. of Alabama, University, Ala. P.O. Box 1444.

Lewis, Brother George. Vice Pres. and Prof., La Salle Coll., Philadelphia 41, Pa.

Lewis, J. H. Dir., Cinemath Tech. Animation Studio, 244 W. 49th St., New York 19, N.Y. 400 W. 118th St., New York 27.

Lewis, Dr. J. v. Math., Supersonic Wind Tunnel Lab., Aberdeen Proving Ground, Md.

Lewis, Sister M. Teresine. Instr., Fontbonne Coll., St. Louis 5, Mo.

Lewis, P. E. Asso. Prof., Oklahoma Agric. and Mech. Coll., Stillwater, Okla. Asso. Prof., State Coll. of Agric. and Eng., Univ. of North Carolina, Rawleigh, N.C.

Lewy, Hans. Prof., Univ. of California, Berkeley 4, Calif.

Li, J. C. R. Asst. Prof., Oregon State Coll., Corvallis, Ore.

Li, T. C. H. Asst. Prof., Drake Univ., Des Moines 11, Iowa. 521 Miller St., Des Moines 15.

Lichnerowicz, André. Prof., Univ. of Strasbourg, Strasbourg, France. 1 Golbery P1.

Lieber, Lillian R. (Mrs. H. G.). Prof., Long Island Univ., Brooklyn 1, N.Y. $624 \mathrm{~A} 3 \mathrm{~d}$ St., Brooklyn 15.

Lieberstein, H. M. Instr., Univ. of Kansas, Lawrence, Kan.

Lifschitz, Dr. Jaime. No address.

Light, F. W. Asst. Prof., Johns Hopkins Univ., Baltimore 18, Md. 719 E. Cold Spring Lane, Baltimore 12. 
Lima Filho, G. S. Asst., Univ. of São Paulo, São Paulo, Brazil.

Lin, C. C. Asso. Prof., Massachusetts Inst. of Tech., Cambridge 39, Mass.

Lindgren, B. W. Instr., Univ. of Minnesota, Minneapolis 14, Minn. 803 9th Ave. S.E.

Lindquist, Dr. C. B. Asso. Prof., Univ. of Minnesota, Duluth 5, Minn.

Lindsay, R. B. Prof., Physics Dept., Brown Univ., Providence 12, R.I.

Linehan, P. H. Prof., City Coll., New York 31, N.Y. 924 West End Ave., New York 25.

Linfield, B. Z. Asso. Prof., Univ. of Virginia, Charlottesville, Va. Hilltop Rd.

Ling, Dr. D. P. Member of Tech. Staff, Bell Telephone Labs., Murray Hill, N.J.

Linial, Samuel. City Coll., New York 31, N.Y. 1775 Clay Ave., New York 57.

Linscheid, H. W. Asso. Prof., Southwestern Inst. of Tech., Weatherford, Okla. 420 N. Caddo St.

Lipschutz, Dr. Miriam A. 755 West End Ave., New York 25, N.Y.

Lipscombe, W. H. Asst. Prof., Univ. of Akron, Akron 4, Ohio.

Lipsich, H. D. Instr., Univ. of Cincinnati, Cincinnati 21, Ohio.

Lister, W. G. Asst. in instruction, Yale Univ., New Haven 11, Conn. Leet Oliver Memorial Hall.

Littell, A. S. Johns Hopkins Univ., Baltimore 18, Md.

Litzinger, Marie. Prof., Mt. Holyoke Coll., South Hadley, Mass.

Livens, G. H. Prof., University Coll., Cardiff, Wales.

Liverman, T. P. G. Univ. of Paris, Paris, France. 28 Berthollet St., Paris 5.

Livers, J. J. Prof., Montana State Coll., Bozeman, Mont.

Livingood, Dr. J. N. B. Aeronautical Research Scientist, Lewis Flight Propulsion Lab., National Advisory Com. for Aeronautics, Cleveland 11, Ohio. 17716 Lakewood Heights Blvd., Lakewood 7, Ohio.

Lockhart, B. J. Asst. Prof., U. S. Naval Postgrad. School, Annapolis, Md.

Loève, Michel. Prof., Univ. of California, Berkeley 4, Calif. 5974 Keith Ave., Oakland, Calif.

Loewner, Charles. Prof., Syracuse Univ., Syracuse 10, N.Y.

Loflin, Z. L. Prof., Southwestern Louisiana Inst., Lafayette, La. Box 353.

Logsdon, Mayme I. (Mrs.). Prof. Emeritus, Univ. of Chicago, Chicago 37, I11.; Prof., Univ. of Miami, Coral Gables 34, Fla.

Lohwater, A. J. Fellow, Columbia Univ., New York 27, N.Y. 19 E. 98th St., New York 29.

Lomont, J. S. Fellow, Physics Dept., Purdue Univ., Lafayette, Ind. 615 North St.

Longenecker, J. V. S. Actuary, Farmers and Bankers Life Ins. Co., Box 580, Wichita, Kan.

Longley, W. R. Prof., Yale Univ., New Haven 11, Conn. 305 Lawrence St.

Lonseth, A. T. Asso. Prof., Oregon State Coll., Corvallis, Ore.

Loo, Dr. C. T. Fellow, Stat. Lab., Univ. of California, Berkeley 4, Calif.

Loomis, L. H. Asso. Prof., Harvard Univ., Cambridge 38, Mass. 17 Selwyn Rd., Belmont 78, Mass.

Lorch, E. R. Prof., Barnard Coll., Columbia Univ., New York 27, N.Y. 501 W. 121 st St.

Lorch, Dr. Lee. Instr., City Coll., New York 31, N.Y. 651 E. 41st St., New York 9.

Lorell, Jack. Research Eng., Jet Propulsion Lab., California Inst. of Tech., Pasadena 4, Calif. 3024 Highview Ave., Altadena, Calif.

Lorenz, A. J. Asst. Prof., St. Louis Univ., St. Louis 3, Mo.

Lorenz, E. N. Staff Member (Meteorology), Massachusetts Inst. of Tech., Cambridge 39, Mass. 420 Memorial Dr.

Loring, S. J. Eng. Consultant, 29 Stiles St., Stratford, Conn.

de Losada y Puga, Cristóbal. Minister of Public Education of Peru; Prof., Catholic Univ. of Peru, Lima, Peru. Apartado 2708.

Lotka, Dr. A. J. Asst. Stat., Metropolitan Life Ins. Co., 1 Madison Ave., New York 10, N.Y.

Loud, W. S. Asst. Prof., Univ. of Minnesota, Minneapolis 14, Minn. 119 Folwell Hall.

Love, C. E. Prof. Emeritus, Univ. of Michigan, Ann Arbor, Mich. 1915 Scottwood. 
$\dagger$ Lovett, E. O. Pres. Emeritus, Rice Inst., Houston 1, Tex.

Lowan, Dr. A. N. Chief, Computation Lab., National Bur. of Standards, 150 Nassau St., New York 7, N.Y.; Prof., Yeshiva Univ., New York 33.

Lowe, R. B. Instr., Polytech. Inst. of Brooklyn, Brooklyn 2, N.Y.

Lowell, S. C. Inst. for Math. and Mech., New York Univ., New York 3, N.Y.

Lowenstein, L. L. Prof., Kent State Univ., Kent, Ohio. 410 Stow St.

Lu, Chin-Hua L. (Mrs. A. C.). 13 Chuan Pan Hutung, Mside Ha Te Men, Peiping, China.

Lubben, R. G. Asso. Prof., Univ. of Texas, Austin 12, Tex. 16 Waggener Hall.

Lubin, C. I. Asso. Prof., UUniv. of Cincinnati, Cincinnati 21, Ohio. 3612 Washington Ave., Cincinnati 29.

Luchins, Edith H. (Mrs. A. S.). Instr., Brooklyn Coll., Brooklyn 10, N.Y. 2052 77th St., Brooklyn 14.

Luckey, R. R. Asso. Prof., Houghton Coll., Houghton, N.Y.

Ludwig, Eleanor A. Instr., Syracuse Univ., Syracuse 10, N.Y. 120 Stinard Ave., Syracuse 4.

Lufkin, Dr. H. M. Instr., Univ. of Pennsylvania, Philadelphia 4, Pa. Bennett Hall.

Lukacs, Eugene. Prof., Our Lady of Cincinnati Coll., Cincinnati 6, Ohio. Stat., Naval Ordnance Test Station, China Lake, Calif. 200-B Halsey, China Lake.

Lund, J. L. Teacher, Woodland High School, Woodland, Calif. 24A 2d St.

Luneburg, Dr. R. K. Inst. for Math. and Mech., New York Univ., New York 3 , N.Y.

Lunn, A. C. Prof. Emeritus, Univ. of Chicago, Chicago 37, I11. 5211 S. Kenwood Ave., Chicago 15.

Luther, C. F. Prof., Willamette Univ., Salem, Ore.

Luxenberg, Harold. Teaching Asst., Univ. of California at Los Angeles, Los Angeles 24, Calif. 1401 N. Catalina St., Los Angeles 27.

Iyles, A. D. Instr., Presbyterian Junior Coll., Maxton, N.C. Box 37.

Lynch, Rev. B. A. Prof., Siena Coll., Loudonville, N.Y.

Lynch, Rev. Joseph. Prof., Physics Dept., Fordham Univ., New York 58, N.Y. Lyndon, Dr. R. C. Instr., Princeton Univ., Princeton, N.J.

Lytle, R. A. Adj. Prof., Univ. of South Carolina, Columbia 19, S.C. 2849 Sheffield Rd., Columbia 4.

McAdam, J. E. Yale Univ., New Haven 11, Conn. 2734 Yale Station.

McBee, Ethelyne L. Teacher, Falls Church High School, Falls Church, Va. 2126 N. Stafford St., Arlington, Va.

McBrien, V. O. Asst. Prof., Coll. of the Holy Cross, Worcester 3, Mass.

McCallion, W. J. Lect., McMaster Univ., Hamilton, Ont., Canada. 24 Franklin Ave.

McCankie, R. C. Vice Pres. and Actuary, Equitable Life Ins. Co. of Iowa, Des Moines 6, Iowa.

McCarthy, E. D. Asst. Prof., Coll. of Eng., Univ. of Detroit, Detroit 21, Mich.

McCarthy, J. J. Asst. Prof., Physics Dept., Newark Coll., Rutgers Univ., Newark 2, N.J. 128 Marine Ave., Brooklyn 9, N.Y.

McCauley, C. E. Development Eng., P. R. Mallory and Co., Inc., North Tarrytown, N.Y. 131 W. 52d St., Bayonne, N.J.

McClay, D. T. Asst. Prof., Univ. of Vermont and State Agric. Coll., Burlington, $\mathrm{Vt}$.

$\dagger$ McClenon, R. B. Prof., Grinnell Coll., Grinnell, Iowa. 1105 Park St.

McClune, Jessie I. Aeronautical Eng., Research Div., United Aircraft Corp., 400 S. Main St., East Hartford 8, Conn.

McConnell, A. J. Prof., Philosophy Dept., Univ. of Dublin, Dublin, Ireland. 36 Trinity College.

McConnell, H. J. Eng. Rt. 9, Box 2817, Sacramento 16, Calif.

McConnon, Myles. Asst. Prof., Norwich Univ., Northfield, Vt. 82 S. Main St.

McCormick, C. T. Prof., Illinois State Normal Univ., Normal, Ill.

McCormick, J. T. Prof., Coll. of the Holy Cross, Worcester 3, Mass.

McCoy, Dorothy. Prof., Belhaven Coll., Jackson 4, Miss. 
McCoy, N. H. Prof., Smith Coll., Northampton, Mass. 53 Ridgewood Ter.

McCrea, W. H. Prof., Royal Holloway Coll., Univ. of London, Englefield Green, Surrey, England. Holmwood, Larch Ave., Sunninghill, Berks, England.

McCulley, W. S. Asst. Prof., Agric. and Mech. Coll. of Texas, College Station, Tex. P.O. Box 2795.

McCuskey, S. W. Prof., Case Inst. of Tech., Cleveland 6, Ohio.

McDaniel, R. R. Prof., Virginia State Coll., Petersburg, Va.

McDaniel, W. C. Asso. Prof., Southern Illinois Univ., Carbondale, Ill.

McDermott, Eugene. Pres., Geophysical Service, Inc., Dallas, Tex. Box 7045, Dallas 9.

McDonald, Janet, Asst. Prof., Vassar Coll., Poughkeepsie, N.Y.

McDonald, Sophia L. (Mrs.'J. H.). Asso. Prof., Univ. of California, Berkeley 4, Calif. 2818 Webster St., Berkeley 5.

McDonough, Dr. D. L. Teacher, South Philadelphia High School for Boys; Instr., Drexel Inst. of Tech., Philadelphia 4, Pa. 1205 Belfield Ave., Drexel Hill, $\mathrm{Pa}$.

McDougle, Edith A. Instr., Women's Coll., Univ. of Delaware, Newark, Del.

McEwen, G. F. Prof. of Physical Oceanography, Scripps Inst. of Oceanography, Univ. of California, La Jolla, Calif. P.O. Box 109.

McEwen, W. H. Prof., Univ. of Manitoba, Winnipeg, Man., Canada. 29 Rothesay Apts.

McFadden, Leonard. Asso. Prof., Virginia Polytech. Inst., Blacksburg, Va. P.O. Box 161.

McFarlan, L. H. Prof., Univ. of Washington, Seattle 5, Wash.

McFarland, Dora. Prof., Univ. of Oklahoma, Norman, Okla. Faculty Exchange.

McGar, F. H. Instr., Fenn Coll., Cleveland 15, Ohio.

McGaughey, A. W. Asso. Prof., Bradley Univ., Peoria 5, Ill.

McKee, Dr. Ruth S. (Mrs. G. W.). 3607 Brisban St., Harrisburg, Pa.

McKelvey, J. V. Prof., Iowa State Coll. of Agric. and Mech. Arts, Ames, Iowa. 2117 Graeber St.

McKelvey, Martha M. (Mrs. J. V.). 2117 Graeber St., Ames, Iowa.

McKenney, Rev. J. L. Prof., Providence Coll., Providence 8, R.I.

McKenzie, H. C. Asst. Prof., Western State Coll., Gunnison, Colo. P.O. Box 73.

McKinsey, Dr. J. C. C. Design Specialist, Douglas Aircraft Co., 3000 Ocean Park Blvd., Santa Monica, Calif. Dept. G4-9-970.

McKnight, Betty. Instr., Centenary Coll., Shreveport, La.

McLaughlin, J. E. Asst., California Inst. of Tech., Pasadena 4, Calif.

McLaughlin, K. F. Instr., U.S. Naval Acad., Annapolis, Md. 326 N. Johnson, Iowa City, Iowa.

McMahon, F. A. Asst. Prof., Manhattan Coll., New York 63, N.Y. 445 E. Fulton St., Long Beach, N.Y.

McMillan, Dr. Audrey W. (Mrs. Brockway). 9 Pearl St., Summit, N.J.

McMillan, Dr. Brockway. Member of Tech. Staff, Bell Telephone Labs., Murray Hill, N.J. 9 Pearl St., Summit, N.J.

McMillan, O. T. Instr., Michigan State Coll., East Lansing, Mich. 216 Morrill Hall.

McNeary, S. S. Asst. Prof., Drexel Inst. of Tech., Philadelphia 4, Pa.

McNeil, Sister M. Gertrude. Prof., Seton Hill Coll., Greensburg, Pa.

McShane, E. J. Prof., Univ. of Virginia, Charlottesville, Va. 209 Maury Ave.

MacCheyne, G. W. Mech. Eng., Stromberg-Carlson Co., Rochester 3, N.Y. 82 Grove St., Geneva, N.Y.

MacColl, Dr. L. A. Member of Tech. Staff, Bell Telephone Labs., Inc., 463 West St., New York 14, N.Y.

MacCullough, R. H. Prof., Defiance Coll., Defiance, Ohio. Sisson Hall.

MacDonald, J. K. L. Asst. Prof., Cooper Union, New York 3, N.Y.; Prof., New York Univ., New York 12. 11 Waverly P1., New York 3.

MacDougal, H. B. Prof., South Dakota State Coll., Brookings, S.D.

MacDuffee, C. C. Prof., Univ. of Wisconsin, Madison 6, Wis. 1815 Summit Ave., Madison 5.

Machado, Dr. E. A. Univ. of LaPlata, LaPlata, Argentina. 73 Gascon, Buenos Aires, Argentina. 
Macintyre, Dr. A. J. Lect., Univ. of Aberdeen, Aberdeen, Scotland. 8 Braemar P1.

Mack, Dr. C. E. Research Eng., Grumman Aircraft Eng. Corp., Bethpage, N.Y. Plant No. 5.

MacKenzie, R. E. Princeton Univ., Princeton, N.J. Graduate College.

Mackey, G. W. Asso. Prof., Harvard Univ., Cambridge 38, Mass. Kirkland House K-21.

Mackie, E. L. Dean of Students and Prof., Univ. of North Carolina, Chapel Hill, N.C. 702 Gimghoul Rd.

Mackin, B. L. Prof., East Central State Coll., Ada, Okla.

MacKinnon, W. D. Actuary, Equitable Life Ins. Co. of Iowa, Des Moines 6, Iowa.

MacLane, G. R. Asst. Prof., Rice Inst., Houston 1, Tex.

MacLane, Saunders. Prof., Univ. of Chicago, Chicago 37, Ill. Eckhart Hall.

MacNair, Peter. Harvard Univ., Cambridge 38, Mass. 54 Perkins Hall.

MacNeille, Dr. H. M. Chief, Fundamental Research Branch, Atomic Energy Comm., Washington 25, D.C. 153 26th St. N.W., Washington 7.

MacNeish, H. F. Visiting Prof., Univ. of Miami, Coral Gables 34, Fla. Hotel Dallas Park, Miami 30, Fla.

MacNerney, J. S. Instr., Univ. of Texas, Austin 12, Tex. 2501 San Gabriel, Austin.

MacPhail, M. S. Asso. Prof., Carleton Coll., Ottawa, Ont., Canada. 142 Brighton Ave.

MacQueen, M. L. Prof., Southwestern Coll., Memphis 12, Tenn.

Maddaus, Dr. Ingo. 81 Columbia Heights, Brooklyn 2, N.Y.

Madden, Sister Teresa M. Prof., Coll. of Our Lady of the Elms, Chicopee, Mass.

Madow, W. G. Prof., Inst. of Stat., Univ. of North Carolina, Chapel Hill, N.C. P.O. Box 168.

Magee, G. R. Asso. Prof., Univ. of Western Ontario, London, Ont., Canada.

Makarov, A. G. Asst. Prof., Rutgers Univ., New Brunswick, N.J.

Maker, Dr. P. T. 56 Franklin St., Wrentham, Mass.

Malde, H. E. 224017 th St., Boulder, Colo.

Mallory, V. S. Prof., New Jersey State Teachers Coll., Upper Montclair, N.J.

Maloney, Sister M. Michael. Prof., D’Youville Coll., Buffalo 1, N.Y.

Malti, M. G. Prof., Elec. Eng. Dept., Cornell Univ., Ithaca, N.Y. Franklin Hall.

Maly, Dis. Asso. Prof., Rensselaer Polytech. Inst., Troy, N.Y.

Manchester, R. E. Dean of Men, Kent State Univ., Kent, Ohio.

Mancib, A. S. Consulting Eng., Alvin S. Mancib Co., 26 Wallace St., West Sommerville, Mass. 46 Yale St., Winchester, Mass.

Mancill, J. D. Prof., Univ. of Alabama, University, Ala.

Mandelbrojt, Szolem. Prof., Coll. of France, Paris, France; Prof., Rice Inst., Houston 1, Tex.

Manger, W. P. Asst., Massachusetts Inst. of Tech., Cambridge 39, Mass 96 Bay State Rd., Boston 15, Mass.

Mann, G. J. Asst. Prof., U.S. Naval Acad., Annapolis, Md. 10 N. Locust Ave.

Mann, H. B. Prof., Ohio State Univ., Columbus 10, Ohio.

Mann, W. R. Teaching Asst., Univ. of California, Berkeley 4, Calif. 2333 Channing Way.

Manning, A. W. Prof., Concordia Collegiate Inst., Bronxville 8, N.Y. 5 Concordia P1.

† Manning, H. P. Prof. Emeritus, Brown Univ., Providence 12, R.I.

Manning, Rhoda. Asst. Prof., Oregon State Coll., Corvallis, Ore.

Manning, W. A. Prof. Emeritus, Stanford Univ., Stanford University, Calif. 649 Alvarado Row.

Mannos, Murray. Asst. Prof., Johns Hopkins Univ., Baltimore 18, Md.

Mansfield, Ralph. Vice Pres. in charge of Eng., Auto-Test, Inc., 1452 S. Michigan Ave., Chicago 5, I11. 8049 S. Maryland Ave., Chicago 19.

Many, Anna E. Prof., Newcomb Memorial Coll., Tulane Univ. of Louisiana, New Orleans 18, La.

Maple, C. G. Asso. Prof., North Texas State Teachers Coll., Denton, Tex. 1124 W. Oak St. 
Marano, J. A. Instr., Manhattan Coll., New York 63, N.Y. 2238 Bassford Ave., New York 57.

Marcaccio, William. Asst. Prof., Xavier Univ., Cincinnati 7, Ohio.

March, H. W. Prof., Univ. of Wisconsin, Madison 6, Wis.; Math., U.S. Forest Products Lab., Madison.

Marchand, E. W. Instr., Univ. of Rochester, Rochester 3, N.Y.

Marcou, Dr. R. J. Math. Physicist, Raytheon Mfg. Co., Waltham, Mass. 930 Beacon St., Newton Centre 59, Mass.

Marden, Morris. Prof., Univ. of Wisconsin in Milwaukee, Milwaukee 3, Wis. 403 E. Carlisle Ave., Milwaukee 11.

de Maré, Mary C. (Mrs. B. L.). Math., Aberdeen Proving Ground, Md. $627 \mathrm{Bel}$ Air Ave., Aberdeen, Md.

Maria, A. J. Asst. Prof., Brooklyn Coll., Brooklyn 10, N.Y.

Maria, Dr. May H. (Mrs. A. J.). Brooklyn Coll., Brooklyn 10, N.Y.

Mariani, Dr. Jean. Member, Inst. for Advanced Study, Princeton, N.J.

Mark, Dr. A. M. Instr., Univ. of Wisconsin, Madison 6, Wis.

Mark, Dr. J. C. Staff Member, Theoretical Physics Div., Los Alamos Scientific Lab., Univ. of California, P.O. Box 1663, Los Alamos, N.M.

Markow, Carl. Instr., St. Peter's Coll., Jersey City 6, N.J. 3836 Bailey Ave., New York 63, N.Y.

Marm, Anna. Prof., Bethany Coll., Lindsborg, Kan. 741 N. 2d St.

Marquis, R. H. Prof., Ohio Univ., Athens, Ohio. P.O. Box 216.

Marriott, R. W. Prof., Swarthmore Coll., Swarthmore, Pa. 213 Lafayette Ave.

Marsaglia, George. Ohio State Univ., Columbus 10, Ohio.

Marshall, J. W. Physicist, U.S. Rubber Co., Ft. Wayne, Ind.

Marshall, Milton. Prof., Physics Dept., Brigham Young Univ., Provo, Utah. 211 S. University Ave.

Marshall, William. Prof. Emeritus, Purdue Univ., Lafayette, Ind. Route 9.

Marston, Dr. A. E. Research Math., Naval Research Lab., Washington 20, D.C. 4320 3d St. S.E., Washington 20.

Marth, Ella. Dean of Women and Asst. Prof., Harris Teachers Coll., St. Louis 12, Mo. 4521a Clarence Ave., St. Louis, 15.

Martin, A. V. 5714 Matilija Ave., Van Nuys, Calif.

Martin, J. E. Instr., Johnstown Center, Univ. of Pittsburgh, Johnstown, Pa. P.O. Box 211.

Martin, M. H. Prof., Univ. of Maryland, College Park, Md.

Martin, Rev. Norbert. Instr., St. Francis Preparatory School, Spring Grove, Pa.

Martin, W. A. Asst. Prof., Georgia School of Tech., Atlanta 1, Ga. Bldg. 16, 251 10th St. N.W., Atlanta.

Martin, W. T. Prof., Massachusetts Inst. of Tech., Cambridge 39, Mass.

Martinson, Margaret E. Instr., Washburn Municipal Univ. of Topeka, Topeka, Kan.

Martyn, W. J. Otago Boys' High School, Dunedin, New Zealand. 16 Sheen St., Roslyn, New Zealand.

Martynes, G. W. L. D. Univ. of Brazil, Rio de Janeiro, Brazil. 598 Atlantic Ave.

Marx, Imanuel. Research Asst., New York Univ., New York 3, N.Y. 219 W. 16th St., New York 11.

Masani, Dr. P. R. Member, Inst. for Advanced Study, Princeton, N.J.

† Mason, Dr. Max. Chm., Observ. Council, California Inst. of Tech., Pasadena 4, Calif.

Mason, W. E. Asso. Prof., Eng. Dept., Univ. of California at Los Angeles, Los Angeles 24, Calif.

Massera, J. L. Prof., School of Eng., Univ. of Montevideo, Montevideo, Uruguay.

Massey, F. J. Asst. Prof., Univ. of Oregon, Eugene, Ore.

Massey, w. S. Princeton Univ., Princeton, N.J.

Matchett, Dr. Margaret S. (Mrs. G. J.). Instr., Illinois Inst. of Tech., Chicago 16, I11. 1551 E. 65th St., Chicago 37.

Mathews, Dr. C. W. Instr., Washington Univ., St. Louis 5, Mo.

Mathewson, L. C. Prof., Dartmouth Coll., Hanover, N.H.

Mathis, H. F. Research Eng., Elec. Eng. Dept., Northwestern Univ., Evanston, III. 
Mauch, Margaret E. Asst. Prof., Univ. of Akron, Akron 4, Ohio.

Mauller, R. A. Asst., Central Coll., Fayette, Mo. 3C Eagle Village.

Mautner, F. I. Massachusetts Inst. of Tech., Cambridge 39, Mass.

Mautner, Frances M. (Mrs. F. I.). 1171 Boylston St., Boston 15, Mass.

May, A. E. Asst. Dir., Univ. of Wisconsin in Racine, Racine, Wis. 1024 Main St.

May, Dr. D. C. Math., Bur. of Ordnance, Navy Dept., Washington 25, D.C. 3410 B St. S.E., Washington 19.

May, Kenneth. Asso. Prof., Carleton Coll., Northfield, Minn.

Mayor, J. R. Asso. Prof., Univ. of Wisconsin, Madison 6, Wis. North Hall.

Maziarz, Rev. E. A. Asst. Prof., Philosophy Dept., St. Joseph's Coll., Collegeville, Ind.

Meacham, E. D. Dean, Coll. of Arts and Sciences, Univ. of Oklahoma, Norman, Okla. Faculty Exch.

Mead, D. G. 22 Fairview Ave., West Orange, N.J.

Mead, Sallie P. (Mrs. C. E. Grant). Member of Tech. Staff, Bell Telephone Labs., Inc., 463 West St., New York 14, N.Y.

Meadows, P. E. Asso. Prof., Carroll Coll., Waukesha, Wis. 100 Racine Ave.

Meals, L. K. Instr., Univ. of Missouri, Columbia, Mo. R.F.D. 3, Gettysburg, Pa.

Mears, Florence M. Prof., George Washington Univ., Washington 6, D.C.

Meder, A. E. Dean, Rutgers Univ., New Brunswick, N.J.

Medina, Alexander. 46 Minerva Ave., Col. Credito Constructor, Mexico, D.F., Mexico.

Mehlenbacher, L. E. Prof., Univ. of Detroit, Detroit 21, Mich. 1207 Longfellow Ave., Royal Oak, Mich.

Mehr, Emanuel. Instr., Johns Hopkins Univ., Baltimore 18, Md.

Meier, Paul. Princeton Univ., Princeton, N.J. Fine Hall.

Melcher, Dr. George. Supt. of Schools Emeritus, Kansas City, Mo. 3331 Campbell St., Kansas City 3.

Melmore, Sidney. 61 Carr Lane, Acomb, York, England.

Melvin, Dr. M. A. Indralaya, East Sound, Wash.

Mendel, C. W. Asso. Prof., Univ. of Illinois, Urbana, Ill.

Mendelsohn, N. S. Asst. Prof., Univ. of Manitoba, Winnipeg, Man., Canada.

Menger, Karl. Prof., Illinois Inst. of Tech., Chicago 16, Ill. 5506 N. Wayne St., Chicago 40.

Mentzer, R. L. Instr., Univ. of Illinois, Urbana, I11. 406 S. Vine St.

Merriell, D. M. Teaching Asst., Univ. of Chicago, Chicago 37, I11. Eckhart Hall.

Merrill, A. S. Prof., Montana State Univ., Missoula, Mont.

† Merrill, Helen A. Prof., Wellesley Coll., Wellesley 81, Mass. 6 Roanoke Rd.

Merriman, G. M. Prof., Univ. of Cincinnati, Cincinnati 21, Ohio.

Merritt, H. L. Instr., Johns Hopkins Univ., Baltimore 18, Md.

Mersman, Dr. W. A. Aeronautical Research Scientist, Ames Aeronautical Lab., National Com. for Aeronautics, Moffet Field, Calif. 2965 Middlefield Rd., Palo Alto, Calif.

Meserve, B. E. Asst. Prof., Univ. of Illinois, Urbana, Ill. 121 W. Franklin.

Métral, Paul. Prof., Lycee of Marseilles, Marseilles, France.

Mette, Andre. Prof., Pasteur Inst., Paris, France. 34 Spintini St., Paris 16.

Mettler, J. W. Instr., Lehigh Univ., Bethlehem, Pa. 1520 W. North St.

Mewborn, A. B. Asso. Prof., U.S. Naval Post-Grad. School, Monterey, Calif. P.O. Box 1748 .

Meyer, B. C. Stanford Univ., Stanford University, Calif.

Meyer, Herman. Prof., Univ. of Miami, Coral Gables 34, Fla.

Meyer, H. A. Asso. Prof., Univ. of Florida, Gainesville, Fla.

Meyer, H. K. Sr. Civil Eng., Eng. Dept., Township of Teaneck, Municipal Bldg., Teaneck, N.J. 562 Center P1.

Meyer, H. L. Asst. Prof., Univ. of Chicago, Chicago 37, I11. 412 Eckhart Hall.

Meyerson, Helen B. (Mrs. M. M.). 29 Washington Sq. W., New York 11, N.Y. Michal, A. D. Prof., California Inst. of Tech., Pasadena 4, Calif.

Michalup, Dr. E. M. Actuary, La Previsora, 37 Este Ave., Caracas, Venezuela.

Michel, R. J. Prof., Southeast Missouri State Teachers Coll., Cape Girardeau, Mo. 225 N. Ellis St.

Michie, J. N. Prof., Texas Tech. Coll., Lubbock, Tex. 2110 17th St. 
Mickelson, Dr. E. L. Instr., Delaware National Guard. Box 1632, Wilmington, Del. Lt. Col., U.S.A.

Mickle, E. J. Asso.'Prof., Ohio State Univ., Columbus 10, Ohio.

Middlemiss, R. R. Prof., Washington Univ., St. Louis 5, Mo.

Mielke, P. T. Asst., Purdue Univ., Lafayette, Ind. F.P.H.A. 517-3.

Miesse, C. C. Research Eng., Battelle Memorial Inst., 505 King Ave., Columbus 1, Ohio.

Mikhail, S. L. Univ. of Illinois, Urbana, Ill. 610 W. Elm St.

Miles, E. J. Asso. Prof., Yale Univ., New Haven 11, Conn. 87 Marvel Rd., New Haven 15.

Miles, E. P. Duke Univ., Durham, N.C. Box 4244, Duke Station.

Miles, H. J. Prof., Univ. of Illinois, Urbana, Ill. 251 Mathematics Bldg.

Milgram, A. N. Asso. Prof., Syracuse Univ., Syracuse 10, N.Y.

Miller, Dr. Abraham. Math., Bur. of Ordnance, Navy Dept., Washington 25, D.C. 4829-BS. 28th St., Arlington, Va.

Miller, Dr. A. L. Treas. and General Manager, Charles N. Miller Co., 100 Massachusetts Ave., Boston 15, Mass.

Miller, B. L. Physicist, Bartol Research Foundation, Franklin Inst., Swarthmore, $\mathrm{Pa}$.

Miller, B. W. Staff Eng., Tele-tronics, Inc., 444 41st St., Miami Beach, Fla. 1220 Pennsylvania Ave.

Miller, Carman E. Prof., Univ. of Saskatchewan, Saskatoon, Sask., Canada.

Miller, Clair E. Teaching Asst., Univ. of Chicago, Chicago 37, I11. 5752 S. Maryland Ave.

Miller, D. D. Asso. Prof., Univ. of Tennessee, Knoxville 16, Tenn. Box 4265, University Station.

Miller, D. S. Asst. Prof., Univ. of Rochester, Rochester 3, N.Y.

Miller, E. A. 113 N. Tioga St., Ithaca, N.Y.

Miller, E. D. Instr., Yuba Coll., Marysville, Calif.

Miller, F. Harshman. B. F. Goodrich Co., 311 E. Central Pkwy., Cincinnati 2, Ohio. 5181 Ralph Ave., Cincinnati 5.

Miller, Frederic H. Prof., Cooper Union, New York 3, N.Y.

$\dagger$ Miller, G. A. Prof., Univ. of Illinois, Urbana, Ill. $1203 \mathrm{~W}$. Illinois St.

Miller, G. T. Asst. Prof., Purdue Univ., Lafayette, Ind. 601 Dodge St.

Miller, Harlan C. Asso. Prof., Texas State Coll. for Women, Denton, Tex. Box 3464.

Miller, Henry C. Asst., Univ. of Alabama, University, Ala. Box 240, Rt. 6, Bessemer, Ala.

Miller, H. L. Prof., Univ. of Cincinnati, Cincinnati 21, Ohio.

Miller, J. L. 542 Georgia Ave., Brooklyn 7, N.Y.

Miller, K. S. Eng., Otis Elevator Co., New York, N.Y. 184 Truman Ave., Yonkers 2, N.Y.

Miller, L. H. Asst. Prof., Ohio State Univ., Columbus 10, Ohio. 1671 Rhoda Ave., Columbus 12.

Miller, Norman. Prof., Queen's Univ., Kingston, Ont., Canada.

Miller, R. A. Asst. Prof., Univ. of Mississippi, University, Miss.

Miller, Rose M. Instr., Vermont Junior Coll., Montpelier, Vt. Glover Hadley Hall.

Miller, W. I. Asso. Prof., Bucknell Univ., Lewisburg, Pa. 220 S. 3d St.

Mills, Dr. M. M. Lect., Aeronautics Dept., California Inst. of Tech., Pasadena 4, Calif. 1565 Kensington Rd., San Marino 9, Calif.

Mills, W. H. Princeton Univ., Princeton, N.J. 460 Riverside Dr., New York 27, N.Y.

Millsaps, Dr. Knox. Ohio State Univ., Columbus 10, Ohio.

Milne, W. E. Prof., Oregon State Coll., Corvallis, Ore.

Milne, Dr. W. P. Retired. 16 Monkbridge Rd., Headingly, Leeds, England.

Miloslavsky, G. C. Princeton Textile Printing Co., 415 E. 32d St., New York 16, N.Y. 600 W. 169 th St., New York 32.

Min, S. H. Asst. Prof., Tsing Hua Univ., Peiping, China.

Minakshisundaram, Dr. S. Reader, Andhra Univ., Waltair, India.

Mindlin, R. D. Prof., Civil Eng. Dept., Columbia Univ., New York 27, N.Y. 45 Christopher St., New York 14. 
Mira, J. A. Prof., Manhattanville Coll. of the Sacred Heart, New York 27, N.Y. 124 E. 84th St., New York 28.

Miranda, Carlo. Prof., Univ. of Naples, Naples, Italy. 31 F Crispi Way.

Mirick, G. R. Asst. Dir., Div. of Teachers Coll. Schools and School Experimentation, and Exec. Dir., Curriculum Service Bur., International Studies, Inc., Teachers Coll., Columbia Univ., New York 27, N.Y. 425 W. 123d St.

Miser, H. J. Asst. Prof., Williams Coll., Williamstown, Mass. Stetson Rd.

Miser, W. L. Prof., Vanderbilt Univ., Nashville 4, Tenn.

Mises, R. v. Prof., Harvard Univ., Cambridge 38, Mass. 10 Chauncy St.

Mishoe, L. I. Asso. Prof., Physics Dept., Morgan State Coll., Baltimore, Md. Mist, H. F. Asst., Univ. of Chicago, Chicago 37, I11. 6824 Ridgeland, Chicago 49.

Mitchell, Dr. A. K. Aerodynamicist, Applied Physics Lab., Johns Hopkins Univ., Silver Spring, Md. 19 Keswick St., Garrett Park, Md.

Mitchell, C. R. Asst. Prof., Rhode Island Coll. of Education, Providence, R.I. 98 Mulberry St., Pawtucket, R.I.

Mitchell, H. B. Chm. of the Board and Treas., Griscom-Russell Co., 285 Madison Ave., New York 17, N.Y. Chapel Farm, New York 63.

Mitchell, Josephine M. Asst. Prof., Univ. of Illinois, Urbana, I11. 365 Mathematics Bldg.

Mitchell, Merle. Asst., Univ. of Wisconsin, Madison 6, Wis. 514 N. Lake, Madison 5 .

Mitchell, Dr. W. L. Aerodynamicist, Bell Aircraft Corp., Buffalo 5, N.Y. 20 Garlow Rd., R.F.D. 1, Lewiston, N.Y.

Mittleman, Don. Instr., Columbia Univ., New York 27, N.Y. 70 Morningside Dr.

Mock, A. J. Teacher, Porterville Junior Coll., Porterville, Calif.

Mode, E. B. Prof., Boston Univ., Boston 15, Mass. 33 Longmeadow Rd., Wellesley 81 , Mass.

Moeller, A. C. Instr., Eng. School, Marquette Univ., Milwaukee 3, Wis.

Moenter, R. L. Asso. Prof., Midland Coll., Fremont, Neb.

Moise, Dr. E. E. Instr., Univ. of Michigan, Ann Arbor, Mich.

Mokre, Johann. Prof., Barat Coll. of the Sacred Heart, Lake Forest, I11.

Molina, E. C. Lect., Newark Coll. of Eng., Newark 2, N.J. 141 Dodd St., East Orange, N.J.

Moll, J. L. Instr., Graduate Center, Ohio State Univ., Wright Field, Ohio. Area (B).

Molloy, Sister M. Corona. Dean, Coll. of Mt. St. Joseph, Mt. St. Joseph, Ohio.

Monsky, Morris. Asst. Actuary, Mutual Life Ins. Co. of New York, 32 Nassau St., New York 5, N.Y.

Montague, Harriet F. Prof., Univ. of Buffalo, Buffalo 14, N.Y.

Monteiro, A. A. Prof., Univ. of Brazil, Rio de Janeiro, Brazil. 882 Almirante Alexandrino Ave.

Montgomery, Dr. Deane. Permanent Member, Inst. for Advanced Study, Princeton, N.J.

Montgomery, J. C. Asso. Prof., Univ. of Connecticut, Storrs, Conn.

Montgomery, P. V. Vice Pres. and Actuary, Southland Life Ins. Co., Southland Life Bldg., Dallas 1, Tex.

Montroll, E. W. Asso. Prof., Univ. of Pittsburgh, Pittsburgh 13, Pa.

Monville, Rev. L. J. Asst. Prof., John Carroll Univ., Cleveland 18, Ohio.

Mood, Dr. A. M. Research Stat., Rand Corp., Douglas Aircraft Co., 1500 4th St., Santa Monica, Calif.

Mooers, C. N. 40 Grozier Rd., Cambridge 38, Mass.

Moon, Parry. Asso. Prof., Elec. Eng. Dept., Massachusetts Inst. of Tech., Cambridge 39, Mass.

Moore, A. H. Instr., Pratt Inst., Brooklyn 5, N.Y. 1585 Unionport Rd., New York 62, N.Y.

Moore, B. C. Asso. Prof., Agric. and Mech. Coll. of Texas, College Station, Tex. P.O. Box 4913.

Moore, C. N. Prof., Univ. of Cincinnati, Cincinnati 21, Ohio. 219 Woolper Ave., Cincinnati 20.

Moore, J. T. Instr., Univ. of Western Ontario, London, Ont., Canada. 
Moore, Dr. Lillian. Teacher, Far Rockaway High School, Far Rockaway, N.Y. 885 N. 28th St., Philadelphia 30, Pa.

Moore, L. T. Asso. Prof., Brooklyn Coll., Brooklyn 10, N.Y. 205 Hicks St., Brooklyn 2.

Moore, M. G. Prof., Bradley Univ., Peoria 5, Ill. 503 W. Melbourne.

Moore, M. R. Instr., Bowling Green State Univ., Bowling Green, Ohio.

† Moore, R. L. Prof., Univ. of Texas, Austin 12, Tex.

Moore, T. W. Asso. Prof., U.S. Naval Acad., Annapolis, Md.

Moore, W. K. Univ. of Kansas, Lawrence, Kan. 417 S. H St., Wellington, Kan.

Moore, W. L. Asso. Prof., Univ, of Louisville, Louisville 8, Ky. Box 59, R.F.D. 2, Coral Ridge, $\mathrm{Ky}$.

Moots, E. E. Prof., Cornell Coll., Mt. Vernon, Iowa. 710 8th Ave. N.

de Moraes, C. A. Rensselaer Polytech. Inst., Troy, N.Y. 42 W. Castle P1., New Rochelle, N.Y.

Morales, J. D. Prof., Univ. of Puerto Rico, Mayagüez, Puerto Rico. 4 Egozcue St., Santurce 34, Puerto Rico.

Moran, Dr. C. W. Instr., Wright Junior Coll., Chicago 34, Ill. 2950 Jarlath St., Chicago 45.

Mordell, L. J. Prof., St. Johns Coll., Cambridge Univ., Cambridge, England.

Morduchow, Dr. Morris. Instr. of Aeronautical Eng., Polytech. Inst. of Brooklyn, Brooklyn 2, N.Y. 1470 Sterling P1., Brooklyn 13.

Morecroft, G. F. Actuarial Clerk, Metropolitan Life Ins. Co., 1 Madison Ave., New York 10, N.Y. 37 Madison Ave.

Morehead, J. C. Prof. Emeritus, Carnegie Inst. of Tech., Pittsburgh 13, Pa. 327 S. College Ave., Salem, Va.

Morenus, Dr. Eugenie M. Retired. Cleveland, N.Y.

Morgan, Dr. F. M. Treas., Clark School Foundation, Hanover, N.H.

Morgan, Dr. Kathryn A. Instr., Syracuse Univ., Syracuse 10, N.Y.

Morgan, W. B. Asst. Prof., Wilmington Coll., Wilmington, Ohio. 615 N. Rombach Ave.

Morgan, W. D. Traffic Analyst, D. L. Piazza Co., 100 N. 7th St., Minneapolis 3, Minn. 1764 St. Anthony Ave., St. Paul 4, Minn.

Morkovin, M. V. Asst. Prof., Aeronautical Eng. Dept., Univ. of Michigan, Ann Arbor, Mich. 2816 Pittsfield Blvd.

Morley, R. K. Prof., Worcester Polytech. Inst., Worcester 2, Mass.

Morrey, C. B. Prof., Univ. of California, Berkeley 4, Calif. 210 Yale Ave., Berkeley 8.

Morrill, W. K. Asso. Prof., Johns Hopkins Univ., Baltimore 18, Md.

Morris, C. R. Math., National Union Radio Corp., Orange, N.J. 421 Lincoln Ave., Orange, N.J.

Morris, F. R. Prof., Fresno State Coll., Fresno 4, Calif.

Morris, Max. Asso. Prof., Case Inst. of Tech., Cleveland 6, Ohio.

Morris, Richard. Prof. Emeritus, Rutgers Univ., New Brunswick, N.J. 12 Johnson St., Highland Park, N.J.

Morris, R. H. Physicist, Color Control Dept., Eastman Kodak Co., Rochester 4, N.Y. 16 Landing Rd. S., Rochester 10.

Morris, T. F. Univ. of Toronto, Toronto 5, Ont., Canada. 198 Ellis Ave., Toronto 3.

Morrison, D. R. Asst., Univ. of Wisconsin, Madison 6, Wis. 2822 Myrtle St., Madison 4.

Morrison. Sister M. Jesse. Instr., Nazareth Coll., Nazareth, Mich.

Morrison, Nathan. Prin. Actuary, New York State Div. of Placement and Unemployment Ins., 342 Madison Ave., New York 17, N.Y. 196 Elm St., New Rochelle, N.Y.

Morrow, D. C. Asso. Prof., Wayne Univ., Detroit 1, Mich.

Morrow, Dorothy J. Biometrician, Medical Service, Civil Aeronautics Admin., Washington 25, D.C. 2115 F St. N.W., Washington 7.

Morrow, R. C. Asso. Prof., U.S. Naval Acad., Annapolis, Md. 102 N. Linden Ave.

Morse, A. P. Asso. Prof., Univ. of California, Berkeley 4, Calif. 18 Oak Vale Ave., Berkeley 5.

Morse, D. S. Prof., Union Coll., Schenectady 8, N.Y. 
Morse, Marston. Prof., Inst. for Advanced Study, Princeton, N.J. Fuld Hall. Morton, J. E. Prof., Stat. Dept., Cornell Univ., Ithaca, N.Y. Warren Hall.

Mosely, M. E. Prof., Jackson Coll., Jackson, Miss. Box 222.

Moser, Catherine E. Instr., Bethany Coll., Bethany, W.Va.

Moser, Leo. Lect., Univ. of Manitoba, Winnipeg, Man., Canada. 436 Cathedral Ave.

Mosesson, Dr. Z. I. Asst. Math., Prudential Ins. Co. of America, 763 Broad St., Newark 1, N.J. 104 N. Munn Ave., East Orange, N.J.

Moskovitz, David. Asso. Prof., Carnegie Inst. of Tech., Pittsburgh 13, Pa.

Mossman, Thirza A. Asso. Prof., Kansas State Coll. of Agric. and Applied Science, Manhattan, Kan.

Mostafa, H. H. Lect., Faculty of Eng., Fouad I Univ., Cairo, Egypt. 2923 James St., Syracuse 6, N.Y.

Mosteller, Frederick. Asso. Prof., Social Relations Dept., Harvard Univ., Cambridge 38, Mass. Emerson Hall.

Mostow, G. D. Member, Inst. for Advanced Study, Princeton, N.J.

Mostow, Wolfe. Math., Bur. of Ships, Navy Dept., Washington 25, D.C. 310 Birch Ave., Takoma Park 12, Md.

Motzkin, Dr. T. S. Instr., Einstein Inst., Hebrew Univ., Jerusalem. International House, Berkeley 4, Calif.

Moulton, E. J. Prof., Northwestern Univ., Evanston, I1l.

Moursund, A. F. Prof., Univ. of Oregon, Eugene, Ore.

Mowbray, A. H. Prof. of Ins., Univ. of California, Berkeley 4, Calif.; Consulting Actuary, Ins. Dept. of California, San Francisco, Calif.

Mowshowitz, Somon. Instr., Univ. of Bridgeport, Bridgeport 5, Conn.

Moye, W. B. Asst. Prof., Georgia Teachers Coll., Collegeboro, Ga.

Moyls, B. N. Asst. Prof., Univ. of British Columbia, Vancouver, B.C., Canada. Hut B-1.

Muhly, H. T. Asst. Prof., U.S. Naval Acad., Annapolis, Md.

Mullen, Rev. Cronan. Prof., Physics Dept., Siena Coll., Loudonville, N.Y.

Muller, Elizabeth M. Instr., Univ. of Bridgeport, Bridgeport 5, Conn.

Mulligan, Sister Charles A. Instr., Albertus Magnus Coll., New Haven 11, Conn.

Mulligan, Dr. J. H. Chief Eng., Television Transmitter Div., Allen B. DuMont Labs., Inc., 1000 Main Ave., Clifton, N.J. 14 Mitchell P1., East Orange, N.J.

Mullings, M. E. Prof., Abilene Christian Coll., Abilene, Tex.

Mullins, E. R. Asst., Univ. of Illinois, Urbana, Ill. 405 W. Springfield Ave., Champaign, Ill.

Mullins, G. W. Prof. Emeritus, Columbia Univ., New York 27, N.Y. 460 Riverside $\mathrm{Dr}$.

Mumford, C. G. Prof., State Coll. of Agric. and Eng., Univ. of North Carolina, Raleigh, N.C. State College Station.

Munro, Dr. G. C. Math., Applied Physics Lab., Johns Hopkins Univ., Silver Spring, Md.

Munro, W. D. Asst. Prof., Univ. of Minnesota, Minneapolis 14, Minn. 39 Clarence Ave. S.E.

Munroe, M. E. Asst. Prof., Univ. of Illinois, Urbana, I11. 360 Mathematics Bldg.

Munshower, C. W. Prof., Colgate Univ., Hamilton, N.Y.

Munster, A. C. Senior Eng., Research Div., Philco Radio and Television Corp., Broad and Somerset Sts., Philadelphia 32, Pa. 264 W. Sulis St., Philadelphia 20.

Murdoch, D. C. Asso. Prof., Univ. of British Columbia, Vancouver, B.C. Canada.

Murdock, W. I. Teaching Fellow, Cornell Univ., Ithaca, N.Y.

Murnaghan, F. D. Visiting Prof., Carnegie Inst. of Tech., Pittsburgh 13, Pa.

Murphy, R. D. Vice Pres. and Actuary, Equitable Life Assurance Society of the U.S., 393 7th Ave., New York 1, N.Y. 28 Godfrey Rd., Upper Montclair, N.J.

Murray, F. J. Asso. Prof., Columbia Univ., New York 27, N.Y. 2 Arden St., New York 34.

Murray, Rev. J. P. Instr., Fairfield Univ., Fairfield, Conn.

Murray, W. R. Prof., Franklin and Marshall Coll., Lancaster, Pa. 
Musselman, J. R. Prof., Western Reserve Univ., Cleveland 6, Ohio.

Myatt, D. J. Asst., Mech. Eng. Dept., Univ. of Louisville, Louisville 8, Ky. 2368 Tyler Lane, Louisville 5.

Myers, A. T. Instr., Univ. of Arizona, Tucson, Ariz. 2812 W. Van Buren, Phoenix, Ariz.

Myers, S. B. Prof., Univ. of Michigan, Ann Arbor, Mich. 3020 Angell Hall.

Nachbin, Leopoldo. Asst. Prof., Univ. of Brazil, Rio de Janeiro, Brazil. 166 Copacabana Ave.

Nagel, Ernest. Prof., Philosophy Dept., Columbia Univ., New York 27, N.Y. Philosophy Hall.

Nagle, J. L. Deputy Commissioner for Real Estate Management, Public Buildings Admin., Federal Works Agency, Washington 25, D.C. Room 6302, Federal Works Bldg.

Nahikian, H. M. Asso. Prof., State Coll. of Agric. and Eng., Univ. of North Carolina, Raleigh, N.C. Box 5548, State College Station.

Nantkes, Elaine V. Visiting Lect., Astronomy Dept., Univ. of Illinois, Urbana, I11.

Nápoles-Gándara, Dr. Alfonso. Dir., Inst. of Math., Univ. of Mexico, Mexico, D.F., Mexico. 204 Mar Negro.

Narayana Row, K. R. 24 Nagasandra Rd., Basavanagudi, Bangalore, India.

Nash, Dr. J. P. Physicist, Kimberly-Clark Corp., Neenah, Wis.

Nathan, Dr. D. S. Instr., City Coll., New York 31, N.Y.

Nead, D. M. Instr., Univ. of Cincinnati, Cincinnati 21, Ohio. Chemistry Bldg.

Neelley, J. H. Prof., Carnegie Inst. of Tech., Pittsburgh 13, Pa. 300 Broadmoor Ave., Pittsburgh 16.

Nef, Walter. Asst. Prof., Univ. of Bern, Bern, Switzerland. 8 St. Marc Way, Fribourg, Switzerland.

Nehari, Zeev. Asso. Prof., Washington Univ., St. Louis 5, Mo. 19 Faculty Lane.

Neifeld, Dr. M. R. Economist, Beneficial Management Corp., 15 Washington St., Newark 2, N.J.

Nelson, A. L. Prof., Wayne Univ., Detroit 1, Mich.

Nelson, C. A. Prof., New Jersey Coll. for Women, Rutgers Univ., New Brunswick, N.J.

Nelson, David. Asst. Prof., George Washington Univ., Washington 6, D.C.

Nelson, J. B. Instr., Los Angeles City Coll., Los Angeles 27, Calif. 3981 Menlo Ave., Los Angeles 37.

Nelson, Dr. Lewis. Senior Math., NEPA Div., Fairchild Engine and Airplane Corp., Box 415, Oak Ridge, Tenn. 109 Glendale Lane.

Nelson, Sara L. Prof., Georgia State Coll. for Women, Milledgeville, Ga. 512 N. Columbia St.

Neményi, Dr. P. F. Physicist, Theoretical Subdiv., Research Dept., Naval Ordnance Lab., White Oak, Silver Spring 19, Md. 3015 Legation St. N.W., Washington 15, D.C.

Nering, E. D. Asst. Prof., Univ. of Minnesota, Minneapolis 14, Minn.

Nesbeda, Dr. Paolo. Instr., Catholic Univ. of America, Washington 17, D.C.

Nesbitt, C. J. Asso. Prof., Univ. of Michigan, Ann Arbor, Mich. 3016 Angell Hall.

Ness, Marie M. Asso., Univ. of Minnesota, Minneapolis 14, Minn. 2530 Dupont Ave. S., Minneapolis 5.

Neugebauer, O. E. Prof., Brown Univ., Providence 12, R.I.

Neuman, Sidney. 717 W. 177th St., New York 33, N.Y.

Neumann, Dr. B. H. Lect., Univ. of Manchester, Manchester 13, England. 75 Westbourne Ave., Hull, England.

von Neumann, John. Prof., Inst. for Advanced Study, Princeton, N.J.

Neustadter, S. F. Lect., Univ. of California, Berkeley 4, Calif. Box 55, Wheeler Hall.

Newburgh, J. D. Instr., Massachusetts Inst. of Tech., Cambridge 39, Mass.

Newell, Dr. H. E. Sec. Head, Rocket-Sonde Research Sec., Naval Research Lab., Washington 20, D.C. 8 Melbourne Ave., Silver Spring, Md.

Newhouse, Albert. Asso. Prof., Univ. of Houston, Houston 4, Tex.

Newlander, August, Univ. of Chicago, Chicago 37, I11. 5757 University Ave. 
Newman, J. J. Teaching Fellow, Harvard Univ., Cambridge 38, Mass. 220 W. 107 th St., New York 25, N.Y.

Newman, Morris. Instr., Univ. of Delaware, Newark, Del.

Newman, M. H. A. Prof., Univ. of Manchester, Manchester 13, England.

Newman, Dr. Philip. Physicist, Watson Labs., Air Materiel Command, Red Bank, N.J. 72 Norwood Ave., Elberon, N.J.

Newsom, Dr. C. V. Asst. Commissioner for Higher Education, State Education Dept., Albany 1, N.Y. 50 Euclid Ave., Albany 3.

Newton, Abba V. Asst. Prof., Vassar Coll., Poughkeepsie, N.Y. 12 Collegeview Ave.

Newton, G. A. Retired. Chapman Ranch, Tex.

Neyman, Jerzy. Dir., Stat. Lab., and Prof., Univ. of California, Berkeley 4, Calif.

Nicholl, Dr. Leonard. Research Dir., Kay-Fries Chemicals, Inc., West Haverstraw, N.Y. 68 Sicles Ave., Nyack, N.Y.

Nichols, G. D. Dean, Univ. of Arkansas, Fayetteville, Ark. Box 11, University Station.

Nichols, W. A. Radio Eng., Canadian Broadcasting Corp., P.O. Box 6000, Montreal, Que., Canada.

Nicholson, W. A. Transmission Man, Long Lines Dept., American Telephone and Telegraph Co., 208 N. Caldwell St., Charlotte 2, N.C. 911 S. Tryon St., Charlotte 6.

Nickel, K. N. 129 S. Estelle, Wichita, Kan.

Nickerson, Helen K. (Mrs. W. J.). Instr., Wheaton Coll., Norton, Mass.

Nielsen, Jakob. Prof., Tech. Univ. of Denmark, Copenhagen $\mathrm{K}$, Denmark. Gjorlingsvej 12, Hellerup, Denmark.

Nielsen, Dr. K. L. Senior Math., Research Lab., Naval Ordnance Plant, Indianapolis 6 , Ind.

Nikodým, O. M. Asso. Prof., Kenyon Coll., Gambier, Ohio. Box 135.

Nilson, E. N. Asst. Prof., Trinity Coll., Hartford 6, Conn. Kenmore Rd., Bloomfield, Conn.

Nirenberg, Louis. Research Asst., Inst. for Math. and Mech., New York Univ., New York 3, N.Y.

Niven, Ivan. Asso. Prof., Univ. of Oregon, Eugene, Ore.

Noble, Andrewa R. Asso. Prof., Pacific Univ., Forest Grove, Ore. 337 4th St. S.

Noble, C. A. Prof. Emeritus, Úniv. of California, Berkeley 4, Calif. 2224 Piedmont Ave.

Nodelman, H. M. Senior Instr., Radio Corp. of America Insts., Inc., 350 W. 4th St., New York 14, N.Y.

Nordhaus, E. A. Asst. Prof., Michigan State Coll., East Lansing, Mich.

Nordling, C. G. A. Asst. Prof., Univ. of Connecticut, Storrs, Conn.

Norlie, O. M. Prof., St. Olaf Coll., Northfield, Minn. 818 Forest Ave.

Nørlund, N. E. Prof., Univ. of Copenhagen, Copenhagen $\varnothing$, Denmark. Malmøgade 6.

Norman, P. B. Asst. Prof., Wagner Coll., Staten Island 1, N.Y. 2175 Cedar Ave., New York 53, N.Y.

Norris, M. J. Asst. Prof., Coll. of St. Thomas, St. Paul 1, Minn. 75 N. Cleveland, St. Paul 5.

Norris, Nilan. Asst. Prof., Economics Dept., Hunter Coll., New York 21, N.Y.

Norris, R. E. Dean of Instruction, State Teachers Coll., Milwaukee 11, Wis.

Northam, J. I. Asst. Prof., Kansas State Coll. of Agric. and Applied Science, Manhattan, Kan.

Northcott, J. A. Prof., Columbia Univ., New York 27, N.Y.

Northrop, E. P. Asso. Dean and Asso. Prof., Univ. of Chicago, Chicago 37, Ill. 5464 Cornell Ave., Chicago 15.

Norton, D. A. Asst., Univ. of Wisconsin, Madison 6, Wis.

Norton, K. A. Physicist, Central Radio Propagation Lab., National Bur. of Standards, Commerce Dept., Washington 25, D.C. 4623 Kenmore Dr. N.W., Washington 7.

Norwood, Lawrence. Math., Evans Signal Corps Lab., Ft. Monmouth, N.J. 28 Slocum P1., Long Branch, N.J. 
Novak, Dr. Ilse L. Instr., Wellesley Coll., Wellesley 81, Mass. 37 Hawthorne St., Cambridge 38 , Mass.

Novak, J. D. Asso. Prof., Univ. of South Carolina, Columbia 19, S.C. 802 Poinsettia Rd., Columbia 50.

Novikoff, A. B. J. Fellow, Stanford Univ., Stanford University, Calif.

Nowlan, F. S. Prof., Univ. of British Columbia, Vancouver, B.C., Canada. Visiting Prof., Univ. of Illinois, Chicago 11, Ill.

Nubar, Dr. Yves. Research Asso., New York Univ., New York 53, N.Y. 45 W. 81st St., New York 24.

Nuess, Vivian R. Asst. Prof., Univ. of Illinois, Urbana, Ill. 1201 W. Oregon.

Nusim, Florence. Math., National Research Lab., Navy Dept., Washington 25, D.C. 416 Cattell St., Easton, $\mathrm{Pa}$.

Nyswander, J. A. Asso. Prof., Univ. of Michigan, Ann Arbor, Mich.

Oakley, C. O. Prof., Haverford Coll., Haverford, Pa.

Oberbeck, Dr. T. E. Scientific Analyst, Operations Evaluation Group, Div. of Industrial Cooperation, Massachusetts Inst. of Tech., Cambridge 39, Mass. $2856 \mathrm{~S}$. Buchanan St., Arlington, Va.

Oberg, E. N. Asso. Prof., State Univ. of Iowa, Iowa City, Iowa. 210-A Physics Bldg.

Oboukhoff, N. M. Prof. of Elec. Eng. and Math. Physics, Oklahoma Agric. and Mech. Coll., Stillwater, Okla. 402 Jefferson St.

O'Brien, Sister Catharine M. Dean, Coll. of Mt. St. Vincent, New York 63, N.Y.

O'Callahan, Rev. J. T. Coll. of the Holy Cross, Worcester 3, Mass.

O'Connor, A. R. Instr., Manhattan Coll., New York 63, N.Y. 4274 Napier Ave., New York 66.

O'Connor, Rev. R. E. Prof., Loyola Coll., Montreal 28, Que., Canada.

Odle, Dr. J. W. Research Math., Naval Ordnance Test Station, China Lake, Calif. 2052 Michelson Lab.

O'Donnell, G. A. Dean, Grad. School, Boston Coll., Chestnut Hill 67, Mass.

Ogden, E. B. Prof., Union Coll., Lincoln 6, Neb. 4626 Bancroft.

Ogg, F. C. Prof., Bowling Green State Univ., Bowling Green, Ohio.

Oglesby, E. J. Prof., Univ. of Virginia, Charlottesville, Va. Box 1887, University Station.

Ohnsorg, F. R. 1230 Edmund Ave., St. Paul 4, Minn.

Oister, Charles. Instr., Keystone Junior Coll., La Plume, $\mathrm{Pa}$.

Oldenburger, Rufus. Prof., DePaul Univ., Chicago 1, Ill.

Olds, C. D. Asso. Prof., San Jose State Coll., San Jose, Calif. P.O. Box 462, Los Altos, Calif.

Olds, E. G. Asso. Prof., Carnegie Inst. of Tech., Pittsburgh 13, Pa. 222 Gladstone Rd., Pittsburgh 17.

Oliphant, Elizabeth A. Asst. Prof., Texas Coll. of Arts and Industries, Kingsville, Tex.

Olive, Gloria. Instr., Idaho State Coll., Pocatello, Idaho.

de Oliveira Castro, F. M. Prof., School of Eng., Univ. of Brazil, Rio de Janeiro, Brazil. 24 Alfredo Chaves St., Botafogo, Rio de Janeiro.

de Oliveira Junior, E. L. Prof., Univ. of Brazil, Rio de Janeiro, Brazil. 148 Aarão Reis St.

Ollivier, Arthur. Prof., Mississippi State Coll., State College, Miss. P.O. Box 504.

Ollmann, L. F. Prof., Hofstra Coll., Hempstead, N.Y.

Olmstead, Dr. P. S. Consultant in Stat., Bell Telephone Labs., Murray Hill, N.J. Box 72, Essex Fells, N.J.

Olmsted, J. M. H. Asso. Prof., Univ. of Minnesota, Minneapolis 14, Minn. 100 Malcolm Ave. S.E.

Olshen, Dr. A. C. Vice Pres. and Actuary, West Coast Life Ins. Co., 605 Market St., San Francisco 5, Calif.

Olson, Emma J. Asso. Prof., Kent State Univ., Kent, Ohio. 537 S. Lincoln St.

Olum, Dr. Paul. Fellow, Inst. for Advanced Study, Princeton, N.J.

O'Neill, Sister Anna C. Asso. Prof., Coll. of St. Elizabeth, Convent, N.J.

O'Neill, Anne F. Asst. Prof., Smith Coll., Northampton, Mass. Laura Scales House. 
Opatowski, Izaak. Asst. Prof. of Math. Biology, Univ. of Chicago, Chicago 37, Ill. 1047 W. North Shore Ave., Chicago 26.

Oppenheim, Alexander. Prof., Raffles Coll., Singapore, Straits Settlements.

Ore, Oystein. Prof., Yale Univ., New Haven 11, Conn. 326 Hall of Graduate Studies.

Orloff, Daniel. Teaching Asst., Univ. of Chicago, Chicago 37, I11. 6211 N. Washtenaw Ave., Chicago 45.

Orloff, Hilda M.'(Mrs. Daniel). 6211 N. Washtenaw Ave., Chicago 45, Ill.

Ormsby, E. F. Instr., Union Coll., Schenectady 8, N.Y.

O'Shea, Rev. E. F. Instr., Univ. of Scranton, Scranton 3, Pa.

Osner, H. J. Instr., Modesto Junior Coll., Modesto, Calif. 416 Oak St.

Osterberg, Alice. Instr., Hunter Coll., New York 21, N.Y. 215 E. 23d St., Brooklyn 26, N.Y.

Ostrofsky, Morris. Prof., Duquesne Univ., Pittsburgh 19, Pa.

Ostrom, T. G. Asst. Prof., Montana State Univ., Missoula, Mont.

Ostrow, E. H. Asst., Univ. of Chicago, Chicago 37, Ill. 5303 Woodlawn Ave., Chicago 15.

Ostrowski, A. M. Prof., Univ. of Basel, Basel, Switzerland.

Ott, E. R. Asso. Prof., Rutgers Univ., New Brunswick, N.J. 199 Sterling Dr., Orange, N.J.

Overholtzer, Dr. G. K. Instr., Univ. of Kansas, Lawrence, Kan. 746 Indiana.

Overman, J. R. Dean Emeritus and Prof., Bowling Green State Univ., Bowling Green, Ohio.

Owchar, Margaret. Teaching Asst., Univ. of Minnesota, Minneapolis 14, Minn.

Owen, D. B. Asso., Univ. of Washington, Seattle 5, Wash. 612 W. 85th St., Seattle 7.

Owens, A. J. Teaching Asst., Univ. of Florida, Gainesville, Fla.

Owens, F. W. Prof., Pennsylvania State Coll., State College, Pa. 462 E. Foster Ave.

Owens, Helen B. (Mrs. F. W.). Asst. Prof., Pennsylvania State Coll., State College, Pa. 462 E. Foster Ave.

Owens, O. G. Asst. Prof., Univ. of Wisconsin, Madison 6, Wis.

Oxtoby, J. C. Asso. Prof., Bryn Mawr Coll., Bryn Mawr, Pa. Dalton Hall.

Oxtoby, Jean S. (Mrs. J. C.). Bryn Mawr Coll., Bryn Mawr, Pa. Dalton Hall.

Pace, W. E. Univ. of Virginia, Charlottesville, Va. 2728 Jefferson Park Ave.

Paganelli, Adelaide B. (Mrs. John). Encyclopaedia Britannica, $20 \mathrm{~N}$. Walker Dr., Chicago 6, Ill. 47 Montclair Ave., Montclair, N.J.

Page, Leigh. Prof., Physics Dept., Yale Univ., New Haven 11, Conn.

Paige, Dr. L. J. Instr., Univ. of California at Los Angeles, Los Angeles 24, Calif.

Painter, Dr. N. H. Member of Research Staff, Univ. of Chicago, Chicago 37, I11. 3600 Sheffield Ave., Lot 115, Hammond, Ind.

Pall, Gordon, Prof., Illinois Inst. of Tech., Chicago 16, Ill. 3034 W. Eastwood Ave., Chicago 25.

Palmer, T. P. Asst. Prof., Rose Polytech. Inst., Terre Haute, Ind.

Palmquist, K. L. Asst. Prof., U.S. Naval Acad., Annapolis, Md. 109 Old Crossing Lane.

Papas, Dr. C. H. Fellow, Harvard Univ., Cambridge 38, Mass. 27 Stone Rd., Belmont 78, Mass.

$\dagger$ Paranjpye, Sir Raghunath P. Retired. Poona 4, India.

Park, H. V. Asso. Prof., State Coll. of Agric. and Eng., Univ. of North Carolina, Raleigh, N.C. Box 5548, State College Station.

Park, R. S. Prof., Eastern Kentucky State Teachers Coll., Richmond, Ky. 213 Burnam Ct.

Parke, Dr. N. G. Consulting Physicist, 39 Main St., Concord, Mass. Spencer Brook Rd.

Parker, Bob. Asst. Prof., Texas Tech. Coll., Lubbock, Tex. 2606 28th St.

Parker, F. D. Instr., Case Inst. of Tech., Cleveland 6, Ohio.

Parker, S. T. Asso. Prof., Kansas State Coll. of Agric. and Applied Science, Manhattan, Kan.

Parker, W. V. Prof., Univ. of Georgia, Athens, Ga. 
Parkinson, G. A. Dir., Milwaukee Extension Div., Univ. of Wisconsin, Milwaukee 3, Wis. 623 W. State St.

Parrish, H. C. Instr., Ohio State Univ., Columbus 10, Ohio. 72 E. Blake Ave., Columbus 2.

Parson, R. H. Acting Chm., Div. of Natural Sciences and Math., Houston Coll. for Negroes, Univ. of Houston, Houston 4, Tex.

Parsons, C. H. 611 W. 112 St., New York 25, N.Y.

Partington, C. R. Instr., Emory Univ., Emory University, Ga.

Partlow, B. W. Asso. Prof., Chemistry Dept., Madison Coll., Harrisonburg, Va. Box 518.

Paschal, Rosa C. Prof., Anderson Coll., Anderson, S.C.

Pate, R. S. Prof., Michigan State Normal Coll., Ypsilanti, Mich.

Patten, W. E. Hydrologist, Flood Control Surveys, Soil Conservation Service, Agric. Dept., Spartanburg, S.C. 1304 Main St., South Boston, Va.

Patterson, G. W. Research Asso., Moore School of Elec. Eng., Univ. of Pennsylvania, Philadelphia 4, Pa. 623 Yale Ave., Morton, Pa.

Patterson, J. M. Instr., Wayne Univ., Detroit 1, Mich.

Patterson, J. O. Retired. 16 W. Lynnwood Ave., Glenside, Pa.

Pauc, Dr. C. Y. Lect., Univ. of Cape Town, Cape Town, South Africa.

Paulson, Edward. Asst. Prof., Univ. of Washington, Seattle 5, Wash.

Paxson, Dr. E. W. Research Math., Rand Corp., Douglas Aircraft Co., 1500 4th St., Santa Monica, Calif.

Paxton, E. K. Asso. Prof., Physics Dept., Virginia Military Inst., Lexington, Va. P.O. Box 754.

Payne, C. K. Asso. Prof., New York Univ., New York 3, N.Y. 25 Valley Rd., Butler, N.J.

Payne, L. E. Fellow, Iowa State Coll. of Agric. and Mech. Arts, Ames, Iowa. $238 \mathrm{~N}$. Hyland.

Payne, Mary H. (Mrs. W. T.). Asst. Prof., Michigan State Coll., East Lansing, Mich. 133 Woodmere Ave.

Peabody, Elizabeth. 201 S. Willson, Bozeman, Mont.

Peabody, Dr. Mary K. Instr., Smith Coll., Northampton, Mass. Laura Scales House.

Peach, M. O. Instr., Carnegie Inst. of Tech., Pittsburgh 13, Pa. 1138 Cloverfield Dr., Pittsburgh 10.

Peacock, Thelma E. Instr., Wells Coll., Aurora, N.Y.

Peak, Philip. Instr., Indiana Univ., Bloomington, Ind.

Pearson, Erna H. (Mrs.). 2522 Mason St., Houston 6, Tex.

Pease, D. K. Asst., Brown Univ., Providence 12, R.I.

Pease, E. M. J. Prof., Rhode Island State Coll., Kingston, R.I.

Peck, E. R. Asst. Prof., Physics Dept., Northwestern Univ., Evanston, I11. 2735 Park P1.

Peck, J. E. L. Yale Univ., New Haven 11, Conn.

Peck, L. C. Instr., Ohio Univ., Athens, Ohio. Edge Hill Apartments.

Peck, L. G. Asst. Prof., Johns Hopkins Univ., Baltimore 18, Md.

Pedigo, Dr. Lois K. (Mrs. C. L.). 2300 Lexington Rd., Louisville 6, Ky.

Pedoe, Dr. Daniel. Lect., Westfield Coll., Univ. of London, Hampstead, London, N.W. 3, England.

Peebles, Dr. G. H. Research Math., Rand Corp., Douglas Aircraft Co., 1500 4th St., Santa Monica, Calif. 3091/2 S. Swall Dr., Los Angeles 36, Calif.

Pegram, Annie M. Retired. 308 Buchanan Blvd., Durham, N.C.

Pehrson, E. W. Prof. Emeritus, Univ. of Utah, Salt Lake City 1, Utah. 1456 Kensington Ave., Salt Lake City 5.

Peiser, Dr. A. M. Math., Hydrocarbon Research, Inc., 115 Broadway, New York 6, N.Y.

Peixoto, Marilia C. (Mrs. M. M.). Asst. Prof., National School of Eng., Rio de Janeiro, Brazil. 93 Palmeiras, Botofogo, Rio de Janeiro.

Peixoto, M. M. Asst. Prof., National School of Eng., Rio de Janeiro, Brazil. 93 Palmeiras, Batofogo, Rio de Janeiro.

Pekeris, C. L. Prof., Weizmann Inst., Rehovoth, Israel. 7 Goodman Rd., Princeton, N.J. 
Pell-Wheeler, Anna (Mrs. A. L. Wheeler). Prof. Emeritus, Bryn Mawr Coll., Bryn Mawr, Pa. 2605 Haste St., Berkeley 4, Calif.

Pence, Sallie E. Asso. Prof., Univ. of Kentucky, Lexington 29, Ky.

Penez, Jacqueline. Brown Univ., Providence 12, R.I. 61 Morton Ave., Woonsocket, R.I.

Penico, A. J. Instr., Univ. of Pennsylvania, Philadelphia 4, Pa. 853 N. 5th St., Philadelphia 23.

Penney, W. F. Cryptanalyst, Navy Dept., Washington 25, D.C. 1506 17th St. N.W.

Pepper, Echo D. Asst. Prof., Univ. of Illinois, Urbana, Ill. 365 Mathematics Bldg.

Pepper, P. M. Asso. Prof., Univ. of Notre Dame, Notre Dame, Ind. Box 1394.

Pepper, R. I. Asst. Prof., Winthrop Coll., Rock Hill, S.C. P.O. Box 1014.

Perkins, F. W. Prof., Dartmouth Coll., Hanover, N.H. 8 Prospect St.

Perlin, I. E. Asso. Prof., Georgia School of Tech., Atlanta 1, Ga. 251 10th St. N.W., Atlanta.

Perlis, Sam. Asst. Prof., Purdue Univ., Lafayette, Ind.

Perlstein, Mae. 1772 Vyse Ave., New York 60, N.Y.

Perrine, Dr. C. D. Retired. Villa Gral. Mitre, Totoral, Cordoba, Argentina.

Perrott, I. B. Lect., Univ. of Leeds, Leeds 2, England. 17 Widney Manor Rd., Solihull, Birmingham, England.

Perry, C. L. Fellow, Univ. of Michigan, Ann Arbor Mich. 1356 Geddes.

Perry, N. C. Lect., Univ. of Southern California, Los Angeles 7, Calif. 3801 S. Grand Ave., Los Angeles 37.

Pérsico, H. A. Prof., Univ. of LaPlata, LaPlata, Argentina. 1795 Juramento, Buenos Aires, Argentina.

Peters, A. S. Asso. Prof., New York Univ., New York 53, N.Y. 602 Rockland Ave., Mamaroneck, N.Y.

Peters, J. W. Asso. Prof., Univ. of Illinois, Urbana, Ill. 253 Mathematics Bldg.

Peters, Ruth M. Asso. Prof., St. Lawrence Univ., Canton, N.Y.

Peters, S. E. Teacher, Long Beach City Coll., Long Beach 8, Calif. 667 Los Altos Ave., Long Beach 14.

Petersen, Richard. Prof., Tech. Univ. of Denmark, Copenhagen K, Denmark. Mynstersvej 9, II, Copenhagen V.

Peterson, J. K. Asst. Prof., Western Michigan Coll., Kalamazoo 45, Mich. 49 W. South St., Kalamazoo 46.

Peterson, O. J. Prof., Kansas State Teachers Coll., Emporia, Kan.

Peterson, R. P. Math., Inst. for Numerical Analysis, National Bur. of Standards, Univ. of California at Los Angeles, Los Angeles 24, Calif.

Peterson, T. S. Asso. Prof., Univ. of Oregon, Eugene, Ore.

Petrie, G. W. Special Eng., Bethlehem Steel Co., Bethlehem, Pa. 1439 Lehigh Pkwy., Allentown, Pa.

Pettis, B. J. Asso. Prof., Tulane Univ. of Louisiana, New Orleans 15, La. Gibson Hall.

Pettis, C. R. Prof., Mississippi State Coll., State College, Miss. P.O. Box 1067.

Pettit, H. P. Prof., Marquette Univ., Milwaukee 3, Wis. Waterford, Wis.

Pflaum, C. W. Teacher, Darrow School, New Lebanon, N.Y.

Pfluger, Albert. Prof., Swiss Federal School of Tech., Zürich, Switzerland. 7 Büchnerstrasse, Zürich 6.

Phalen, H. R. Prof., Coll. of William and Mary, Williamsburg, Va. 130 Chandler Ct.

Phelps, C. R. Asst. Prof., Rutgers Univ., New Brunswick, N.J.

Phelps, R. J. Junior Eng., Labs. Div., Teleregister Corp., 157 Chambers St., New York 7, N.Y.

Phibbs, Edgar. Asst. Prof., Univ. of Alberta, Edmonton, Alta., Canada. 9859 70th Ave.

Phillips, C. A. Asst., Univ. of Illinois, Urbana, Ill. 367 Mathematics Bldg.

Phillips, H. B. Prof. Emeritus, Massachusetts Inst. of Tech., Cambridge 39, Mass.

Phillips, R. S. Asso. Prof., Univ. of Southern California, Los Angeles 7, Calif.

Picone, Mauro. Prof., Univ. of Rome, Rome, Italy. 18 Tre Madonne Way. 
Pierce, W. A. Harvard Univ., Cambridge 38, Mass. 39 Trowbridge St.

Piersol, C. C. Math., Naval Ordnance Lab., White Oak, Silver Spring 19, Md. 7007 Aspen Ave., Takoma Park 12, Md.

Pihl, Dr. Mogens. Lect., Vestre Borgerdydskole, Danmarks Loverhojskole, Copenhagen K, Denmark. 24 Hoeghsmindevej, Gentofte, Denmark.

Pinette, Lucille $\mathbf{K}$. Asst. Prof., Colby Coll., Waterville, Me.

Pinl, M. J. Prof., Univ. of Cologne, Cologne, Germany. Oberrahmede Kreis A1tena Westfalen, 70 Neuen Weg, British Occupation Zone, Germany.

Pinney, Edmund. Asst. Prof., Univ. of California, Berkeley 4, Calif.

Piper, R. I. Personnel Asst., Pacific Telephone and Telegraph Co., $140 \mathrm{New}$ Montgomery St., San Francisco 5, Calif. Route 2309.

Pipes, L. A. Asso. Prof., Eng. Dept., Univ. of California at Los Angeles, Los Angeles 24, Calif. 2471 Walgrove Ave., Venice, Calif.

Piranian, George. Asst. Prof., Univ., of Michigan, Ann Arbor, Mich.

Pitcairn, Joel. Instr., Temple Univ., Philadelphia 22, $\mathrm{Pa}$. Bryn Athyn, $\mathrm{Pa}$.

Pitcher, Everett. Prof., Lehigh Univ., Bethlehem, Pa. 422 W. Broad St.

Pitt, H. R. Prof., Queen's Univ., Belfast, Northern Ireland.

Pitts, R. J. Prof., Ft. Valley State Coll., Ft. Valley, Ga.

Pitts, Walter, Massachusetts Inst. of Tech., Cambridge 39, Mass.

Pixley, Dr. Emily C. (Mrs. H. H.). 20194 Briarcliff Rd., Detroit 21, Mich.

Pixley, H. H. Asst. Dean, Coll. of Liberal Arts., and Asso. Prof., Wayne Univ., Detroit 1, Mich.

Plancherel, Michel. Prof., Swiss Federal School of Tech., Zürich, Switzerland.

Plant, L. C. Retired. 231 Oakhill Ave., East Lansing, Mich.

Pleijel, A. V. C. Docent, Lund Univ., Lund, Sweden. Inst. for Advanced Study, Princeton, N.J.

Pocock, J. G. Instr., Milton Acad., Milton 86, Mass.

Podmele, Theresa L. Teacher, East High School, Buffalo 8, N.Y. 356 Lisbon Ave., Buffalo 15.

Pohle, F. V. Instr., Inst. for Math. and Mech., New York Univ., New York 3, N.Y.

Poitras, Rev. Albeni. Dean of Students, St. Joseph's Univ., St. Joseph's, N.B., Canada.

van der Pol, Balthasar. Dir., Advisory Com. for Radio Communications. 24 Parklaan, Eindhoven, Netherlands.

Polachek, Dr. Harry. Math., Naval Ordnance Lab., White Oak, Silver Spring 19, Md.; Asst. Prof., Univ. of Maryland, College Park, Md. 3279 S. Stafford St., Arlington, Va.

Pollak, H. O. Harvard Univ., Cambridge 38, Mass. 8 Terrace P1., Stamford, Conn.

Pollard, Harry. Asso. Prof., Cornell Univ., Ithaca, N.Y. 112 Auburn St.

Pollard, H. S. Prof., Miami Univ., Oxford, Ohio. 350 Patterson Ave.

Polley, J. C. Prof., Wabash Coll., Crawfordsville, Ind.

Pollock, Helen S. Instr., West Virginia Univ., Morgantown, W.Va. 300 Glendon Ave.

P6lya, George. Prof., Stanford Univ., Stanford University, Calif. 660 Dartmouth St., Palo Alto, Calif.

Ponds, J. W. Asst. Prof., West Virginia State Coll., Institute, W.Va.

Poole, A. R. Asst. Prof., Oregon State Coll., Corvallis, Ore.

Poor, C. L. Prof. Emeritus, Columbia Univ., New York 27, N.Y. Dering Harbor, Shelter Island Heights, N.Y.

Poor, V. C. Asso. Prof. Emeritus, Univ. of Michigan, Ann Arbor, Mich. 930 Packard St.

Poritsky, Dr. Hillel. General Elec. Co., 1 River Rd., Schenectady 5, N.Y. 1012 Wendell Ave., Schenectady 8.

Porter, E. A. Sec. and Actuary, Manhattan Life Ins. Co., 120 W. 57th St., New York 19, N.Y. 17 Glen Ridge Pkwy., Bloomfield, N.J.'

Porter, Goldie H. (Mrs. M. B.). Asst. Prof., Univ, of Texas, Austin 12, Tex. 2402 Windsor Rd., Austin 21.

Porter, J. W. Math., Naval Air Developmental Station, Johnsville, Pa. 1260 N. 54th St., Philadelphia 31, Pa. 
Porter, M. B. Prof. Emeritus, Univ. of Texas, Austin 12, Tex. 2402 Windsor Rd., Austin 21.

Porter, Ruth E. Instr., Berea Coll., Berea, Ky. Box 1251.

Post, E. L. Asso. Prof., City Coll., New York 31, N.Y. 610 W. 173d St., New York 32.

Potor, Valentina A. Asst. Prof., Youngstown Coll., Youngstown, Ohio. 474 Coitsville Rd., Campbell, Ohio.

Pott, F. H. Aeronautical Research Scientist, Flight Propulsion Research Lab., National Advisory Com. for Aeronautics, Cleveland, Ohio. 12547 Lake Ave., Lakewood 7, Ohio.

Potts, D. H. Asst. Prof., Northwestern Univ., Evanston, I11.

Pounder, D. W. Asst., Illinois Inst. of Tech., Chicago 16, Ill.

Pounder, I. R. Prof., Univ. of Toronto, Toronto 5, Ont., Canada.

Povey, Kenneth. Lib., Queen's Univ., Belfast, Northern Ireland.

Powell, J. E. Prof., Michigan State Coll., East Lansing, Mich. 137 Bogue St.

$\dagger$ Powers, R. E. Retired. 225 E. 6th St., Pomona, Calif.

Prager, William. Prof., Brown Univ., Providence 12, R.I.

Preiser, Rabbi T. H. 30 Tudela St., Jerusalem.

Prendergast, H. B. Lect., Univ. of Manitoba, Winnipeg, Man., Canada. 276 Wellington Crescent.

Prenowitz, Walter. Asso. Prof., Brooklyn Coll., Brooklyn 10, N.Y. 501 W. 121st St., New York 27.

Preston, Bernard. 103 Park Ave., New York 17, N.Y.

Pretz, P. S. Prof., St. Benedict's Coll., Atchison, Kan.

Price, G. B. Prof., Univ. of Kansas, Lawrence, Kan. 205 Frank Strong Hall.

Price, H. H. Prof., Pasadena Coll., Pasadena 7, Calif. 1799 N. Hill Ave.

Price, H. V. Asso. Prof., State Univ. of Iowa, Iowa City, Iowa. University High School.

Price, J. F. Instr., Oregon State Coll., Corvallis, Ore.

Prim, R. C. Research Asso., Princeton Univ., Princeton, N.J.

Proctor, W. W. Asst. Prof., Morgan State Coll., Baltimore 12, Md.

Proschan, Frank. Asso., George Washinton Univ., Washington 6, D.C. 2133 F St. N.W., Washington 7.

Protter, M. H. Asst. Prof., Syracuse Univ., Syracuse 10, N.Y.

Pruett, Lee D. (Mrs. J. R.). Teaching Fellow, Indiana Univ., Bloomington, Ind.

Puckett, W. T. Asso. Prof., Univ. of California at Los Angeles, Los Angeles 24, Calif. Inst. for Advanced Study, Princeton, N.J.

Pulliam, F. M. Asst. Prof., Univ. of Kentucky, Lexington 29, Ky. 114 Arcadia Park, Lexington 2.

Purcell, E. J. Prof., Univ. of Arizona, Tucson, Ariz.

Putnam, A. L. Asst. Prof., Univ. of Chicago, Chicago 37, Ill. Eckhart Hall 413.

Putnam, Dr. C. R. Instr., Johns Hopkins Univ., Baltimore 18, Md. 3504 Hayward Ave., Baltimore 15.

Putnam, R. G. Prof., New York Univ., New York, N.Y. 115 Riverview Ave., Tarrytown, N.Y.

Putt, C. N. Asst., Kansas State Coll. of Agric. and Applied Science, Manhattan, Kan. 2500 Granthurst, Topeka, Kan.

Putz, R. R. Teaching Asst., Univ. of California, Berkeley 4, Calif. 1631 Cornell Ave., Berkeley 2.

Pyle, H. R. Prof., Whittier Coll., Whittier, Calif.

Quade, E. S. 2621 30th St., Santa Monica, Calif.

Quaid, L. J. Asst. Prof., Univ. of Minnesota, Minneapolis 14, Minn. 208 Engineering Bidg.

Quarles, D. A. Asst. in Instruction, Yale Univ., New Haven 11, Conn. 273 Robin Rd., Englewood, N.J.

Quarles, H. L. Lt. Col. A.U.S., Hqs. 7th Inf. Div., APO 7, San Francisco, Calif.

Querry, J. W. Prof., Sam Houston State Teachers Coll., Huntsville, Tex. 1313 21 st St.

Quinn, Dr. Grace S. (Mrs. R. B.). 3221 Wheeler Rd. S.E., Washington 20, D.C.

Quinn, Brother Leo. Prof., St. Francis Coll., Brooklyn 2, N.Y. 
Quintella, A. N. M. Prof., Military Coll., Rio de Janeiro, Brazil. 16 Santa Terezinha St.

Rabson, Gustave. Univ. of Michigan, Ann Arbor, Mich. 934 Dewey.

Rademacher, Hans. Prof., Univ. of Pennsylvania, Philadelphia 4, Pa. Bennett Hall.

Rader, C. B. Asst. Prof., Univ. of Houston, Houston 4, Tex. 5533 McCormick, Houston 3.

Radin, Arthur. Tutor, Drafting Dept., City Coll, New York 31, N.Y. 1055 Findlay Ave., New York 56.

Rado, Tibor. Prof., Ohio State Univ., Columbus 10, Ohio. 92 Walhalla Rd., Columbus 2.

Rådström, H. V. Instr., Univ. of Stockholm, Stockholm, Sweden. 8 Sikrenovàgen, Stocksund, Sweden.

Rahn, Edris P. Teacher, Hayward Union High School, Hayward, Calif. 1456 Glen Dr., San Leandro, Calif.

Rainich, G. Y. Prof., Univ. of Michigan, Ann Arbor, Mich. 602 Oswego St.

Rainville, E. D. Asso. Prof., Univ. of Michigan, Ann Arbor, Mich. 1459 Rosewood.

Raisbeck, Gordon. Instr., Massachusetts Inst. of Tech., Cambridge 39, Mass.

Raleigh, John. Univ. of Pennsylvania, Philadelphia 4, Pa. 2300 S. 11th St., Philadelphia 48.

Rambo, Susan M. Prof. Emeritus, Smith Coll., Northampton, Mass. 71 Ridgewood Ter.

Ramler, O. J. Prof., Catholic Univ. of America, Washington 17, D.C. 12 Girard St. N.E., Washington 2.

Ramsey, Margaret. Asso. Prof., Linfield Coll., McMinnville, Ore. P.O. Box 449.

Randels, Dr. W. C. Research Eng., North American Aviation, Inc., Inglewood, Calif. 6009 Mansfield St., Los Angeles 43, Calif.

Randolph, J. F. Prof., Univ. of Rochester, Rochester 3, N.Y. Morey Hall.

Raney, G. N. Lect., Columbia Univ., New York 27, N.Y. 59 E. 7th St., New York 3.

Rankin, J. M. Prof., Coll. of Idaho, Caldwell, Idaho. 1810 Ash St.

Rankin, Dr. R. A. Lect., Univ. of Cambridge, Cambridge, England. Memorial Court, Clare Coll.

Rao, B. S. M. Prof., Central Coll., Univ. of Mysore, Bangalore, India.

Rao, K. N. Lib., Annamalai Univ., Annamalainagar, India.

Rapoport, Anatol. Asst. Prof., Univ. of Chicago, Chicago 37, I11. Com. on Math. Biology.

Rapp, Mary K. Instr., Illinois Inst. of Tech., Chicago 16, I11. 5804 Giddings St., Chicago 30.

Rasch, Dr. G. W. Lect., State Serum Inst., Univ. of Copenhagen, Copenhagen S, Denmark.

Rasmusen, Dr. Ruth B. Instr., Wilson Junior Coll., Chicago 21, I11. 6105 Woodlawn Ave., Chicago 37.

Rasmussen, O. M. Instr., Univ. of Kansas. Lawrence, Kan. 814 E. 13th St.

Rasor, S. E. Prof. Emeritus, Ohio State Univ., Columbus 10, Ohio. 1594 Neill Ave., Columbus 1.

Ratner, L. T. Teaching Asst., Univ. of California at Los Angeles, Los Angeles 24, Calif. 152 S. Maple Dr., Beverly Hills, Calif.

Rauch, L. L. Instr., Princeton 'Univ., Princeton, N.J. Fine Hall.

Raudenbush, H. W. Asso. Prof., Queens Coll., Flushing, N.Y.

Rawhouser, Robert. Math., Wright Field, Ohio. 65 W. Great Miami Blvd., Dayton 5, Ohio.

Rawling, A. G. Asst., Univ. of Maryland, College Park, Md. P.O. Box 331.

Rawlins, C. H. Prof., U.S. Naval Postgrad. School, Annapolis, Md. 13 Franklin St.

Raybould, Ethel H. Lect., Univ. of Queensland, Brisbane, Queensland, Australia.

Rayl, Adrienne S. Asso. Prof., Birmingham Center, Univ. of Alabama, Birmingham, Ala. 2825 Thornhill Rd., Birmingham 5.

Raymond, Rev. John. Prof., St. Martin's Coll., Olympia, Wash. 
Raynor, G. E. Prof., Lehigh Univ., Bethlehem, Pa. 349 8th Ave.

Reade, M. O. Asst. Prof., Univ, of Michigan, Ann Arbor, Mich. 275 West Engineering Bldg.

Reagan, L. M. Asso. Prof., Univ. of Wichita, Wichita, Kan. 1056 N. Market St., Wichita 5.

Reaves, S. W. Prof., Univ. of Oklahoma, Norman, Okla. 527 Chautauqua Ave.

Rechard, O. H. Dean, Univ. of Wyoming, Laramie, Wyo.

Rechard, Dr. O. W. Instr., Ohio State Univ., Columbus 10, Ohio.

$\dagger$ Reddick, H. W. Prof., New York Univ., New York 53, N.Y. Box 205.

Redheffer, Dr. R. M. Instr., Harvard Univ., Cambridge 38, Mass. 424 Beacon St., Boston 15, Mass.

Reed, F. W. Prof., Ohio Univ., Athens, Ohio. 61 Columbia Ave.

Reed, I. S. Consultant, Northrup Aircraft, Hawthorne, Calif. 2050 Emporer Ave., Temple City, Calif.

Reed, Dr. L. J. Vice Pres., Johns Hopkins Univ., Baltimore 18, Md. 615 N. Wolfe St.

Reed, S. G. Instr., Physics Dept., Georgetown Univ., Washington 7, D.C. 170 U St. N.E., Washington 2.

Rees, C. J. Prof., Univ. of Delaware, Newark, Del.

Rees, Dr. Mina S. Head, Math. Branch, Office of Naval Research, Washington 25, D.C. 1117 N. Pitt St., Alexandria, Va.

Rees, P. K. Asso. Prof., Louisiana State Univ. and Agric. and Mech. Coll., Baton Rouge 3, La. 307 Centenary Dr., Baton Rouge 14.

Regan, Francis. Prof., St. Louis Univ., St. Louis 3, Mo.

Rehberg, C. F. Asso. Prof. of Elec. Eng., New York Univ., New York 53, N.Y. 25-28 84th St., Flushing, N.Y.

Reichelderfer, P. V. Asso. Prof., Ohio State Univ., Columbus 10, Ohio. 328 E. Beechwold Blvd., Columbus 2.

Reid, W. P. Asst. Prof., Purdue Univ., Lafayette, Ind.

Reid, W. T. Prof., Northwestern Univ., Evanston, I11.

Reiner, Irving. Asst. Prof., Univ. of Illinois, Urbana, I11.

Reiner, Dr. Irma M. (Mrs. Irving). Instr., Univ. of Illinois, Urbana, Ill.

Reingold, Haim. Asso. Prof., Illinois Inst. of Tech., Chicago 16, Ill.

Reinsch, B. P. Prof., Florida Southern Coll., Lakeland, Fla. 191 Lake Morton Dr.

Reissner, Eric. Asso. Prof., Massachusetts Inst. of Tech., Cambridge 39, Mass.

Reiter, Hans. Asst., Rice Inst., Houston 1, Tex.

Reklis, Dr. Virginia M. (Mrs. E. P.). 35 Defense Dr., Aberdeen, Md.

Remage, Russell. Instr., Univ. of Delaware, Newark, Del. Instr., Úniv. of Pennsylvania, Philadelphia 4, Pa. 4819 Regent St., Philadelphia 43.

Rempfer, Dr. R. W. Math. Physicist, Farrand Optical Co., Bronx Blvd. and E. 238th St., New York 66, N.Y. 20 Prospect Ave., Ardsley, N.Y.

Renner, Theresa M. Instr., Univ. of Arkansas, Fayetteville, Ark. Room 116, B.A.

Renno, J. G. Fellow, Univ. of Wisconsin, Madison 6, Wis. 140 E. Johnson St., Madison 3.

Renzetti, Dr. N. A. Physicist, Research, Development, and Test Organization, Naval Ordnance Test Station, China Lake, Calif.

Resch, Daniel. Instr., Syracuse Univ., Syracuse 10, N.Y.

Reschovsky, Helene. Asso. Prof., Russell Sage Coll., Troy, N.Y. 1996 15th St.

Resnikoff, M. M. Instr., Pennsylvania State Coll., State College, Pa. 1414 Washington Ave., Altoona, $\mathrm{Pa}$.

Reynolds, C. N. Prof., West Virginia Univ., Morgantown, W.Va. 217 McLane Ave.

Reynolds, R. R. Asst. Prof., Oklahoma Agric. and Mech. Coll., Stillwater, Okla. Navy Project, Harvard Univ., Cambridge 38, Mass. 223 Pierce Hall.

Reynolds, Dr. T. D. Instr., Duke Univiv., Durham, N.C. Box 4646, Duke Station.

Rhodes, E. E. Vice Pres., Mutual Benefit Life Ins. Co., Box 359, Newark 1, N.J. 233 Elwood Ave., Newark 4.

Rhodes, J. S. Sampling Stat., Bur. of the Census, Commerce Dept., Washington 25, D.C. 1201 Valley Ave. S.E., Washington 20.

Ribeiro, Dr. Hugo. Lect., Univ. of California, Berkeley 4, Calif. 
Riblet, Dr. H. J. Radio Eng., Submarine Signal Co., 160 N. Washington St., Boston 14, Mass. 3 Eliot Rd., Belmont 78, Mass.

Ricabarra, R. A. Univ. of LaPlata, LaPlata, Argentina. 683 53d St.

Rice, Harris. Prof., Worcester Polytech. Inst., Worcester 2, Mass.

Rice, J. N. Asso. Prof., Catholic Univ. of America, Washington 17, D.C. 3326 13th St. N.E.

Rice, Sister M. Edmund. Business Manager, Our Lady of Cincinnati Coll., Cincinnati 6 , Ohio.

Rice, R. B. Senior Physicist, Research Dept., Phillips Petroleum Co., Bartlesville, Okla.

Rich, Dr. G. R. Dir., Chas. T. Main, Inc., 80 Federal St., Boston 10, Mass.

Rich, R. P. Johns Hopkins Univ., Baltimore 18, Md. 303 E. 29th St.

Richardson, D. P. Prof., Univ. of Arkansas, Fayetteville Ark. 524 Leverett St.

Richardson, Moses. Asst. Prof., Brooklyn Coll., Brooklyn 10, N.Y.

$\dagger$ Richardson, R. G. D. Dean Emeritus and Prof. Emeritus, Brown Univ., Providence 12, R.I.

Richert, D. H. Prof. Emeritus, Bethel Coll., North Newton, Kan.

Richmond, D. E. Prof., Williams Coll., Williamstown, Mass. 11 Park St.

Rickart, C. E. Asst. Prof., Yale Univ., New Haven 11, Conn. 1050 Yale Station.

Ricker, Dr. Norman. Senior Research Physicist, Research Lab., Carter Oil Co., P.O. Box 801, Tulsa 2, Okla. 1718 E. 30th St., Tulsa 5.

Rickey, F. A. Prof., Louisiana State Univ. and Agric. and Mech. Coll., Baton Rouge 3, La.

Rider, P. R. Prof., Washington Univ., St. Louis 5, Mo.

Ridgway, A. O. Asst., Univ. of Maryland, College Park, Md.

Riess, J. K. Asst. Prof., Physics Dept., Tulane Univ. of Louisiana, New Orleans 15 , La. 17 Audubon Blvd.

Rigby, Dr. F. D. Math., Office of Naval Research, Washington 25, D.C. 1505 Tyler Ave., Tyler Park, Falls Church, Va.

Riggs, L. G. Instr., Northwestern Univ., Evanston, I11. 1725 Orrington Ave., Apt. 731 .

Riley, J. D. Math., Naval Research Lab., Washington 20, D.C. 3958 2d St. S.W.

Riley, Melita H. (Mrs. C. W.). Stat., United Nations, Lake Success, N.Y. 147-27 Charter Rd., Jamaica 2, N.Y.

Rinehart, Dr. R. F. Dir., Planning Div., Research and Development Board, Pentagon Bldg., Washington 25, D.C. Room 3E572.

Rines, David. Lawyer, 40 Broad St., Boston 9, Mass.

Ringenberg, L. A. Prof., Eastern Illinois State Coll., Charleston, Ill.

Riordan, John. Member of Tech. Staff, Bell Telephone Labs., Inc., 463 West St., New York 14, N.Y.

Ripton, T. B. 242-31'51st Ave., Flushing, N.Y.

Ritt, J. F. Prof., Columbia Univ., New York 27, N.Y.

Ritt, R. K. Instr., Univ. of Michigan, Ann Arbor, Mich.

Ritter, E. K. Asso. Prof., U.S. Naval Postgrad. School, Annapolis, Md.

Ritter, I. F. Asso. Prof., New York Univ., New York 53, N.Y.

Ritterman, M. B. Instr., Long Island Univ., Brooklyn 1, N.Y. 7201 Ridge Blvd., Brooklyn 9.

Rivers, M. Ethyl. Instr., Univ. of Tennessee, Knoxville 16, Tenn. 1841 Laurel Ave.

Robbins, C. K. Asso. Prof., Purdue Univ., Lafayette, Ind. 418 Vine St., West Lafayette, Ind.

Robbins, H. E. Asso. Prof., Inst. of Stat., Univ. of North Carolina, Chapel Hill, N.C. P.O. Box 168.

Robbins, Dr. R. B. Retired. 1443 Gentry Lane, Charlottesville, Va.

Roberson, R. E. Senior Research Eng., Mech. Dept., Washington Univ., St. Louis 5, Mo. 4549 Magnolia, St. Louis 10.

Roberts, A. E. 651 Highland Ave., Revloc, Pa.

Roberts, Helen M. (Mrs. S. C.). Asst. Prof., Univ. of Connecticut, Storrs, Conn.

Roberts, J. H. Prof., Duke Univ., Durham, N.C. Box 4987.

Roberts, R. A. Harvard Univ., Cambridge 38, Mass. 127 Mt. Auburn St.

Roberts, Virginia B. Instr., Texas Tech. Coll., Lubbock, Tex. 220814 th St. 
Robertson, H. P. Prof. of Math. Physics, California Inst. of Tech., Pasadena 4, Calif. Norman Bridge Lab.

Robertson, M. S. Asso. Prof., Rutgers Univ., New Brunswick, N.J. 16 Lenox Ave., Box 339, Milltown, N.J.

Robinson, G. de B. Asso. Prof., Univ. of Toronto, Toronto 5, Ont., Canada.

Robinson, H. A. Prof., Agnes Scott Coll., Decatur, Ga.

Robinson, Joan E. Bryn Mawr Coll., Bryn Mawr, Pa.

Robinson, Dr. Julia B. (Mrs. R. M.). 2533A Dwight Way, Berkeley 4, Calif.

Robinson, L. B. 131 E. North Ave., Baltimore 2, Md.

Robinson, L. V. Asso. Prof., Univ. of South Carolina, Columbia 19, S.C.

Robinson, P. G. Asso. Prof., Iowa State Coll. of Agric. and Mech. Arts, Ames, Iowa.

Robinson, Robin. Prof., Dartmouth Coll., Hanover, N.H. 16 Allen St.

Robinson, R. M. Asso. Prof., Univ. of California, Berkeley 4, Calif.

Robinson, S. L. Asst. Prof., City Coll., New York 31, N.Y. Hudson View Gardens, New York 33.

Robinson, v. G. Asst., Purdue Univ., Lafayette, Ind.

Robinson, V. N. Asso. Prof., U.S. Naval Acad., Annapolis, Md. Lt. Com., U.S.N.

Robinson, W. J. Prof., Centre Coll. of Kentucky, Danville, Ky.

Robison, G. M. Asso. Prof., Susquehanna Univ., Selinsgrove, Pa.

Robusto, C. C. Asst. Prof., St. Johns Univ., Brooklyn 2, N.Y.

Rocha, P. S. Prof. of Theoretical Mech., Univ. of Brazil, Rio de Janeiro, Brazil. 28 Aires de Saldanha St., Copacabana, Rio de Janeiro.

Rochford, Sister M. DePazzi. Prof., Briar Cliff Coll., Sioux City 17, Iowa.

Rochmes, Nathan. Instr., Univ. of Illinois, Urbana, Ill. 205 S. Busey Ave.

Rock, D. H. Asst, Prof., Iowa State Coll. of Agric. and Mech. Arts, Ames, Iowa.

Rodabaugh, L. D. Asso. Prof., Southern Illinois Univ., Carbondale, I11. 709 W. College.

Rodgers, T. G. Dean Emeritus, New Mexico Highlands Univ., Las Vegas, N.M. 1018 4th St.

Roessler, E. B. Asso. Prof., Coll. of Agric., Univ. of California, Davis, Calif.

Roettinger-Kaplan, Dr. Ida (Mrs. Wilfred Kaplan). 1308 Olivia Ave., Ann Arbor, Mich.

Roever, W. H. Prof. Emeritus, Washington Univ., St. Louis 5, Mo. 6802 Waterman Ave.

Roger, Frédéric. Prof., Univ. of Bordeaux, Bordeaux, France. 51 Park St., Cauderan, France.

Rolfe, Kathryn B. (Mrs. R. W.). Asso., Coll. of Agric., Univ. of California, Davis, Calif.

Rommel, Dr. J. D. 1724 Edenside Ave., Louisville 4, Ky.

Room, T. G. Prof., Univ. of Sydney, Sydney, New South Wales, Australia. Visiting Prof., Univ. of Washington, Seattle 5, Wash.

Roos, Dr. C. F. Pres., Econometric Inst., Inc., 500 5th Ave., New York 18, N.Y. 817 5th Ave., New York 21.

Roosevelt, G. E. Roosevelt and Son, 30 Pine St., New York 5, N.Y.

Root, R. E. Prof. Emeritus, U.S. Naval Postgrad. School, Annapolis, Md. 7 Franklin St.

Roscoe, Marguerite K. Instr., Montana State Coll., Bozeman, Mont. 209 Ave. B, Billings, Mont.

Rose, G. F. Instr., Univ. of Wisconsin, Madison 6, Wis. 111 N. Randall Ave., Madison 5.

Rose, I. H. Asst. Prof., Univ. of Massachusetts, Amherst, Mass.

Rose, M. E. New York Univ., New York, N.Y. 106 Webster St., Irvington 11, N.J.

Rosen, Saul. Lect., Univ. of California at Los Angeles, Los Angeles 24, Calif.

Rosenbach, J. B. Prof., Carnegie Inst. of Tech., Pittsburgh 13, Pa. 2550 Beechwood Blvd., Pittsburgh 17.

Rosenbaum, Ira. Asst. Prof., Univ. of Miami, Coral Gables 34, Fla. 760 N.W. 13th Ct., Miami, Fla.

Rosenbaum, Louise J. (Mrs. R. A.). Asst. Prof., Reed Coll., Portland 2, Ore.

Rosenbaum, R. A. Asso. Prof., Reed Coll., Portland 2, Ore. 
Rosenbloom, J. H. Asso. Physicist, Experimental Branch, Fire Control Div., Frankford Arsenal, Philadelphia 37, Pa. 343 E. Roosevelt Blvd., Philadelphia 20.

Rosenbloom, P. C. Asst. Prof., Syracuse Univ., Syracuse 10, N.Y.

Rosenlicht, M. A. Teaching Fellow, Harvard Univ., Cambridge 38, Mass. 44 Irving St.

Rosenthal, Arthur. Prof., Purdue Univ., Lafayette, Ind.

Rosenthal, Dr. Jenny E. 226 Bath Ave., Long Branch. N.J.

Rosenthall, Edward. Asso. Prof., McGill Univ., Montreal, Que., Canada. Engineering Bldg.

Ross, A. E. Prof., Univ. of Notre Dame, Notre Dame, Ind.

Ross, Dr. Clarence. Math., Naval Proving Ground, Dahlgren, Va. P.O. Box 94.

Ross, R. M. Asst. Prof., Rose Polytech. Inst., Terre Haute, Ind. Box $32 \mathrm{~A}$, R.F.D. 5.

Ross, V. H. Asst., Univ. of Wisconsin, Madison 6, Wis. 705 Langdon St., Madison 5 .

Rosser, J. B. Prof., Cornell Univ., Ithaca, N.Y. White Hall.

Rosskopf, M. F. Asso. Prof., Syracuse Univ., Syracuse 10, N.Y.

Roth, S. G. Asst. Prof., New York Univ., New York 3, N.Y.

Roth, W. E. Prof., Sampson Coll., Sampson, N.Y. Henry Peacham Apt.

Rothberger, Fritz. Asst. Prof., Acadia Univ., Wolfville, N.S., Canada.

Rothe, E. H. Asst. Prof., Univ. of Michigan, Ann Arbor, Mich. 1015 Vaughn St.

Rothrock, D. A. Prof. Emeritus, Indiana Univ., Bloomington, Ind. 1000 Atwater Ave.

Rothstein, Sally I. Teacher, William Howard Taft High School, New York 57, N.Y. 124 E. 176 St., New York 53.

Roudebush, Dr. W. C. New Mexico Military Inst., Roswell, N.M.

Rowen, Rose G. (Mrs. J. W.). Math., Army Map Service, 6500 Brooks Lane, Washington 25, D.C. 4217 S. 36th St., Arlington, Va.

Rowland, S. A. Prof., Ohio Wesleyan Univ., Delaware, Ohio. 45 Oak Hill Ave.

Rubashkin, Leila Raines. Teaching Fellow, Cornell Univ., Ithaca, N.Y. 123 Highland Place.

Rubenstein, Mollie A. (Mrs. Herbert). 5808 Woodcrest Ave., Philadelphia 31, $\mathrm{Pa}$.

Rubin, Herman. 7142 S. East End Ave., Chicago 49, I1l.

Rubin, Joyce. Instr., Polytech. Inst. of Brooklyn, Brooklyn 2, N.Y.; Teacher, Seward Park High School, New York 2, N.Y. 2119 Valentine Ave., New York 57.

Rubinfien, David. Asst. Research Eng., Illinois Inst. of Tech., Chicago 16, I11. 117 S. Central Ave., Chicago 44.

Rudberg, Hans. Vice Pres., Iron Refining Co., Haelleforsnaes, Sweden.

Ruderfer, Herbert. Univ. of Pennsylvania, Philadelphia 4, Pa. 1588 Sterling P1., Brooklyn 13, N.Y.

Ruderman, H. D. Teacher, Manhattan High School of Aviation Trades, New York 21, N.Y. 1533 Townsend Ave., N.Y. 52.

Rudin, Walter. Duke Univ., Durham, N.C. Box 5173, Duke Station.

Ruley, B. T. Senior Eng., SKF Industries, Inc., Front and Erie, Philadelphia 34, Pa. 1414 The Kenilworth, Alden Park, Philadelphia 44.

Rulon, P. J. Prof., Grad. School of Education, Harvard Univ., Cambridge 38, Mass. 40 Quincy St.

Runge, Lulu L. Asst. Prof., Univ. of Nebraska, Lincoln 8, Neb.

Running, T. R. Prof. Emeritus, Univ. of Michigan, Ann Arbor, Mich. 1019 Michigan Ave.

Ruse, H. S. Prof., Univ. of Leeds, Leeds 2, England.

Rusk, W. J. Retired. 1415 Park St., Grinnell, Iowa.

Russell, Helen G. Asso. Prof., Wellesley Coll., Wellesley 81, Mass.

Russell, W. P. Prof. Emeritus, Pomona Coll., Claremont, Calif. P.O. Box 13.

Rust, Rev. C. H. St. Louis Univ., St. Louis 3, Mo.

Rust, Dr. W. M. Head, Geophysics Research, Humble Oil and Refining Co., P.O. Box 2180, Houston 1, Tex. 
Rutledge, W. A. Instr., Univ. of Tennessee, Knoxville 16, Tenn. 1400 White Ave., Knoxville.

Rutt, N. E. Prof., Louisiana State Univ. and Agric. and Mech. Coll., Baton Rouge 3, La.

Ryan, D. R. Asst. Prof., Gonzaga Univ., Spokane 11, Wash. E. 727 Illinois Ave., Spokane 13.

Ryser, Dr. H. J. Member, Inst. for Advanced Study, Princeton, N.J.

Saalfrank, C. W. Asst. Prof., Rutgers Univ., New Brunswick, N.J.

Sabin, Mary S. Teacher, Denver High School, Denver 3, Colo. 1333 E. 10th Ave.

Sachs, Bernard. Instr., Polytech. Inst. of Brooklyn, Brooklyn 2, N.Y.

Sachs, Dr. J. M. Wilson Junior Coll., Chicago 21, I11.

Sacks, Louis. Instr., Carnegie Inst. of Tech., Pittsburgh 13, Pa.

Sadowsky, M. A. Asso. Prof., Illinois Inst. of Tech., Chicago 16, I11.

$\dagger$ Safford, F. H. Prof. Emeritus, Univ. of Pennsylvania, Philadelphia 4, Pa. 4527 Osage Ave., Philadelphia 43.

Sagastume Berra, A. E. Prof., Univ. of LaPlata, LaPlata, Argentina. 353 47th St.

Saibel, Edward. Prof., Mech. Dept., Carnegie Inst. of Tech., Pittsburgh 13, Pa.

Salem, Raphael. Asso. Prof., Massachusetts Inst. of Tech., Cambridge 39, Mass. Room 2-279.

Salerno, John. Math., U.S. Coast and Geodetic Survey, 641 Washington St., New York 14, N.Y. 530 Lincoln Ave., Brooklyn 8, N.Y.

Salkind, Charles. Teacher, S. J. Tilden High School, Brooklyn 3, N.Y. 1304 New York Ave.

Salkind, William. 127 Remsen St., Brooklyn 2, N.Y.

Saltonstall, W. G. Prin., Phillips Exeter Acad., Exeter, N.H.

Saltzer, Charles. Instr., Case Inst. of Tech., Cleveland 6, Ohio.

Salzer, H. E. Math., Computation Lab., National Bur. of Standards, 150 Nassau St., New York 7, N.Y. 1903 Ocean Ave., Brooklyn 30, N.Y.

Samelson, Hans. Asst. Prof., Univ. of Michigan, Ann Arbor, Mich.

Samuel, Dr. Pierre. Research Asst., National Center for Scientific Research, Paris 7, France. 56 Maubeuge St., Paris 9.

Sanchez-Díaz, Rafael. Prof., Coll. of Agric. and Mech. Arts, Univ. of Puerto Rico, Mayagüez, Puerto Rico.

Sandt, J. E. Asst. Prof., Marietta Coll., Marietta, Ohio.

Sanger, R. G. Prof., Kansas State Coll. of Agric. and Applied Science, Manhattan, Kan.

Sangren, W. C. Fellow, Univ. of Michigan, Ann Arbor, Mich. 1435 University Ter.

Santalo, L. A. Prof., Inst. of Math., Univ. of Rosario, Rosario, Argentina. Inst. for Advanced Study, Princeton, N.J.

Santos, A. M. V. P.O. Box 709, Salvador, Bahia, Brazil.

Sard, Arthur. Asso. Prof., Queens Coll., Flushing, N.Y. 146-19 Beech Ave.

Sarno, A. H. Instr., St. John's Univ., Brooklyn 6, N.Y. 82-10 60th Rd., Elmhurst, N.Y.

Saslaw, S. S. Asso. Prof., U.S. Naval Acad., Annapolis, Md.

Sasuly, Max, Research Asso., Robinson Foundation, 14 Wall St., New York 5, N.Y. 412 5th St. N.W. Washington 1, D.C.

Satterthwaite, Dr. F. E. Design Eng., General Elec. Co., Ft. Wayne, Ind. 4110 Bowser Ave., Ft. Wayne 5.

Saunders, R. B. Asso. Prof., Oregon State Coll., Corvallis, Ore.

Saunders, S. W. Prof., Morgan State Coll., Baltimore 12, Md.

Saunders, T. J. Asso. Prof., Univ. of San Francisco, San Francisco 17, Calif.

Savage, I. R. Columbia Univ., New York 27, N.Y. 1414 John Jay Hall.

Savage, Dr. L. J. Research Asso., Univ. of Chicago, Chicago 37, Ill.

Savit, C. H. Math., Western Geophysical Co., Edison Bldg., Los Angeles 13, Calif. 15323/4 Rosalin Rd., Los Angeles 27.

Sawyer, J. W. Instr., Univ. of Missouri, Columbia, Mo.

Saxer, Walter. Goldbach-Küsnacht, Zürich, Switzerland. 
Scarborough, J. B. Prof., U.S. Naval Acad., Annapolis, Md. Ferry Farms, R.F.D. 2 .

Schaaf, S. A. Asst. Prof., Mech. Eng. Dept., Univ. of California, Berkeley 4, Calif. 229 Mech. J.C.

Schacknow, A. B. Instr., Polytech. Inst. of Brooklyn, Brooklyn 2, N.Y.

Schaefer, G. R. 1602 Elm St., Hays, Kan.

Schaeffer, A. C. Prof., Purdue Univ., Lafayette, Ind.

Schaeffer, Sister M. Rosalin. Ursuline Coll., Louisville 6, Ky.

Schaerf, H. M. Asso. Prof., Washington Univ., St. Louis 5, Mo.

Schafer, Alice T. (Mrs. R. D.). Asst. Prof., Swarthmore Coll., Swarthmore, Pa.

Schafer, R. D. Asst. Prof., Univ. of Pennsylvania, Philadelphia 4, Pa. Bennett Hall.

Schaffer, Sister M. Charles. Instr., Immaculate Heart Coll., Los Angeles 27, Calif.

Schart, Dr. W. J. Research Eng., Consolidated Vultee Aircraft Corp., San Diego, Calif. 4331 Yale Ave., La Mesa, Calif.

Schatten, Robert. Asso. Prof., Univ. of Kansas, Lawrence, Kan.

Schecter, Samuel. Instr., Syracuse Univ., Syracuse 10, N.Y.

Scheffé, Henry. Asso. Prof., Math. Stat. Dept., Columbia Univ., New York 27, N.Y.

Scheier, Rev. M. A. Prof., St. Bonaventure Coll., St. Bonaventure, N.Y.

Schelkunoff, Dr. S. A. Research Math., Bell Telephone Labs., Inc., 463 West St., New York 14, N.Y.

Scherk, Peter. Asso. Prof., Univ. of Saskatchewan, Saskatoon, Sask., Canada.

Schiffer, Dr. M. M. Instr., Inst. of Theoretical Physics, Hebrew Univ., Jerusalem. 201 Pierce Hall, Harvard Univ., Cambridge 38, Mass.

Schild, Albert, Instr., Univ. of Pennsylvania, Philadelphia 4, Pa. 3024 W. Susquehanna Ave., Philadelphia 21.

Schild, Alfred. Asst. Prof., Univ. of Toronto, Toronto 5, Ont., Canada.

Schilling, O. F. G. Asso. Prof., Univ. of Chicago, Chicago 37, I1l. Eckhart Hall.

Schliestett, G. V. Head, Fluid Mech. Branch, Office of Naval Research, Washington 25, D.C. $2700 \mathrm{Q}$ St. N.W., Washington 7.

Schlotthauer, Lilah G. (Mrs. A. D.). Asst. Prof., Walla Walla Coll., College Place, Wash. P.O. Box 353.

Schmied, R. W. Instr., Tulane Univ. of Louisiana, New Orleans 15, La. 2017 Peniston St.

Schneckenburger, Edith R. Asst. Prof., Univ. of Buffalo, Buffalo 14, N.Y.

Schnittke, Raymond. Major, U.S.A. 6156 S. Ellis Ave., Chicago 37, Ill.

Schoenbaum, Emil. Prof., Charles Univ., Prague II, Czechoslovakia. 26 Tneouská, Prague Bubeneč, Czechoslovakia.

Schoenberg, I. J. Prof., Univ. of Pennsylvania, Philadelphia 4, Pa. 333 Dartmouth Ave., Swarthmore, $\mathrm{Pa}$.

Schoenfeld, Lowell. Asst. Prof., Univ. of Illinois, Urbana, I11. 1103 W. Church St., Champaign, Ill.

Scholomiti, N. C. Instr., Univ. of Illinois, Chicago 11, I11. 2200 W. Cullom Ave., Chicago 18.

Scholz, Dr. J. P. Instr., Wilson Coll., Chambersburg, Pa.

Schouten, Dr. J. A. Wissel A 126 IX, Epe, Netherlands.

Schraut, K. C. Prof., Univ. of Dayton, Dayton 9, Ohio.

Schub, Dr. Pincus. Instr., Univ. of Pennsylvania, Philadelphia 4, Pa. State Hospital, Trenton 8, N.J.

Schubert, Dr. Jewell E. Instr., Univ. of Illinois, Urbana, Ill. 605 E. Chalmers St., Champaign, Ill.

Schuld, E. G. Instr., Univ. of Wisconsin in Milwaukee, Milwaukee 3, Wis.

Schumaker, J. A. Instr., MacMurray Coll. for Women, Jacksonville, Ill. $330 \mathrm{~W}$. College Ave.

Schurrer, Augusta L. Asst., Univ. of Wisconsin, Madison 6, Wis. 1731 Regent St., Madison 5.

Schutzberger, Henry. Research Math., Research and Development Div., New Mexico School of Mines, Albuquerque, N.M. 1611 Ridgecrest Circle. 
Schwartz, Abraham. Instr., City Coll., New York 31, N.Y. 196 Bogert Rd., River Edge, N.J.

Schwartz, B. L. Fellow, Brown Univ., Providence 12, R.I. 121 Power St., Providence 6.

Schwartz, Dr. H. M. Scientist, Brookhaven National Lab., Upton, N.Y. 369 Oak St., Patchogue, N.Y.

Schwartz, J. T. 1915 Southern Blvd., New York 60, N.Y.

Schweigert, G. E. Asso. Prof., Univ. of Pennsylvania, Philadelphia 4, Pa. College Hall.

Schweitzer, Dr. A. R. Research Math., 452 Oakdale Ave., Chicago 14, Ill. Box 653, Lake Forest, I11.

Schweitzer, E. O. Elec. Eng., 1241 Waukegan Rd., Northbrook, Ill.

Schwerdtfeger, Dr. H. W. E. Lect., Univ. of Melbourne, Melbourne N. 3, Victoria, Australia.

Scobert, W. G. Instr., Univ. of Oregon, Eugene, Ore.

Scott, Carol S. (Mrs. W. C.). Teacher, St. Petersburg Junior Coll., St. Petersburg 3, Fla. 1190 8th St. N., St. Petersburg 4.

Scott, L. L. Asst., Univ, of Illinois, Urbana, I1l. 910 College Ct.

Scott, Mary L." (Mrs. William Squire). 146 16th St., Buffalo 13, N.Y.

Scott, Dr. W. R. Instr., Univ. of Michigan, Ann Arbor, Mich.

Scott, W. T. Asst. Prof., Northwestern Univ., Evanston, Ill.

Sealander, C. E. Asst. Prof., Ohio State Univ., Columbus 10, Ohio.

Secada, C. F. Lt. Com., Peruvian Navy. 565 Copacabana, Lima, Peru.

Secrist, J. B. Instr., Brooklyn Coll., Brooklyn 10, N.Y. 70 Morningside Dr., New York 27.

Sedgewick, C. H. W. Prof., Univ. of Connecticut, Storrs, Conn.

Seebeck, C. I. Asso. Prof., Univ. of Alabama, University, Ala. Box 814.

Seeber, R. R. Senior Staff Member, Pure Science Dept., International Business Machines Corp., 590 Madison Ave., New York 22, N.Y.

Seekins, C. W. Asso. Prof., U.S. Naval Acad., Annapolis, Md. 107 Old Crossing Lane.

Seely, Dr. Caroline E. Willseyville, Tioga County, N.Y.

Segal, I. E. Asst. Prof., Univ. of Chicago, Chicago 37, Ill. Eckhart Hall.

Segner, Sister M. Georgianne. Dean of Women and Instr., Le Clerc Coll., Belleville, Ill.

Seidel, Wladimir. Prof., Univ. of Rochester, Rochester 3, N.Y. Inst. for Numerical Analysis, Univ. of California at Los Angeles, Los Angeles 24, Calif.

Seidenberg, Abraham. Asst. Prof., Univ. of California, Berkeley 4, Calif. 1900-B Woolsey, Berkeley 3.

Seielstad, H. D. Lt. Com., U.S.C.G., 9th C. G. District, Keith Bldg., Cleveland 15, Ohio. 1676 Chesterland, Lakewood 7, Ohio.

Seiler, Brother Louis De LaSalle. Dean, St. Mary's Coll., Winona, Minn.

Selberg, Atle. Asso. Prof., Syracuse Univ., Syracuse 10, N.Y.

Self, Fariebee P. (Mrs.). Instr., Centenary Coll., Shreveport, La.

Selfridge, O. G. Instr., Massachusetts Inst. of Tech., Cambridge 39, Mass.

Seligman, C. B. Harvard Univ., Cambridge 38, Mass. Kirkland House A-23.

Selz, P. B. Asst. Treas. and Business Manager, Parsons Coll., Fairfield, Iowa. College Campus.

Semple, J. G. Prof., King's Coll., Univ. of London, London W.C. 2, England.

Senior, J. K. Asso. Prof., Univ. of Chicago, Chicago 37, I11. 5612 Kenwood Ave.

Senior, J. L. Vice Pres. and Treas., Lottrell and Senior, Inc., 331 Madison Ave., New York 17, N.Y.

Seth, B. R. Prof., Hindu Coll., Delhi, India.

Seybold, M. Anice. Prof., North Central Coll., Naperville, Ill.

Shaffer, Dorothy B. (Mrs. L. H.). Research Asst., Harvard Univ., Cambridge 38, Mass. 98 Westgate, Cambridge 39.

† Shah, Dr. S. M. Lect., Muslim Univ., Aligarh, India.

Shanks, E. B. Asst. Prof., Vanderbilt Univ., Nashville 4, Tenn.

Shanks, M. E. Asso. Prof., Purdue Univ., Lafayette, Ind. 
Shannon, Dr. C. E. Member of Tech. Staff, Bell Telephone Labs., Murray Hill, N.J.

Shapiro, A. S. Teaching Fellow, Univ. of Michigan, Ann Arbor, Mich. 413-2 Forest Ave.

Shapiro, E. I. Instr., Brooklyn Col1., Brooklyn 10, N.Y. 2865 Ocean Ave., Brooklyn 29.

Shapiro, George. Instr., Johns Hopkins Univ., Baltimore 18, Md.

Shapiro, H. N. Asst. Prof., New York Univ., New York, N.Y. 1704 Seddon St., New York 61.

Shapley, Harlow. Dir., Harvard Coll. Observ., Harvard Univ., Cambridge 38, Mass.

Sharp, H. S. C. Prof., U.S. Coast Guard Acad., New London, Conn.

Sharpe, Dr. J. A. Vice Pres., C. H. Frost Gravimetric Surveys, Inc., Box 58, Tulsa, Okla.

Shaub, H. C. Prof., Washington and Jefferson Coll., Washington, Pa.

Shaw, A. N. Prof., Physics Dept., McGill Univ., Montreal, Que., Canada.

Shaw, R. H. Math., CNO (CNC), Navy Dept., Washington 25, D.C. 117 N. Edgewood St., Arlington, Va.

Shaw, W. F. 1733 Ohio Ave., Mercedes, Tex.

Sheffer, H. M. Prof., Philosophy Dept., Harvard Univ., Cambridge 38, Mass. Emerson Hall.

Sheffer, I. M. Prof., Pennsylvania State Coll., State College, Pa. 212 Sparks Bldg.

Sheffield, E. T. Asst. Prof., Univ. of Alberta, Edmonton, Alta., Canada. H. S. Bldg.

Sheldon, E. W. Prof. Emeritus, Univ. of Alberta, Edmonton, Alta., Canada.

Shenton, W. F. Prof., American Univ., Washington 16, D.C. 3605 Porter St. N.W.

Shephard, R. W. Asst. Prof., New York Univ., New York 3, N.Y.

Shepherd, J. C. Instr., Univ. of Maryland, College Park, Md. 4811 Guilford Rd.

Shepherd, W. L. Instr., Univ. of Oregon, Eugene, Ore.

Sherak, Bernard. Instr., Newark Coll., Rutgers Univ., Newark, N.J.

Sheridan, L. W. Asso. Prof., Coll. of St. Thomas, St. Paul 1, Minn.

Sherman, Bernard. Instr., Univ. of Vermont and State Agric. Coll., Burlington, Vt. Waterman Bldg. UVM.

Sherman, Dr. Jack. Math., Research Labs., Texas Co., Beacon, N.Y. P.O. Box 750, Poughkeepsie, N.Y.

Sherman, Dr. Jacob. Prin. Physicist, Naval Base, Philadelphia, Pa. 3024 S. 23d Ter., Philadelphia 45.

Sherman, Seymour. Member, Inst. for Advanced Study, Princeton, N.J.

Sherman, W. L. 60 Pitman St., Providence 6, R.I.

Sherratt, W. A. Asst. Prof., Presbyterian Coll., Clinton, S.C. P.O. Box 451.

Sherwood, G. E. F. Prof., Univ. of California at Los Angeles, Los Angeles 24, Calif. $400 \mathrm{~N}$. Barrington Ave.

Shewhart, Dr. W. A. Research Eng., Bell Telephone Labs., Murray Hill, N.J. 158 Lake Dr., Mountain Lakes, N.J.

Shi, Miriam E. (Mrs. W. M.). Instr., Alabama Polytech. Inst., Auburn, Ala. Graves Apt. 13-A.

Shields, W. H. Instr., Massachusetts Inst. of Tech., Cambridge 39, Mass. 8620 Yale P1., Philadelphia 36, $\mathrm{Pa}$.

Shiffman, Max. Prof., Stanford Univ., Stanford University, Calif. 1162 Saratoga Ave., Palo Alto, Calif.

Shirk, J. A. G. Prof., Kansas State Teachers Coll., Pittsburg, Kan. 116 E. Lindburg.

Shniad, Dr. Harold. Instr., Purdue Univ., Lafayette, Ind.

Shnider, Ruth W. (Mrs. J. C.). Elec. Eng., Naval Research Lab., Washington 20, D.C. 1833 New Hampshire Ave. N.W., Washington 9.

Sholander, Marlow. Asst. Prof., Washington Univ., St. Louis 5, Mo.

Shonka, E. A. Prof. and Business Manager, St. Procopios Coll., Lisle, I11.

Shook, C. A. Asso. Prof., Lehigh Univ., Bethlehem, Pa. 1122 W. Broad St. 
Shook, Dr. R. C. Vice Pres., International Stat. Bur., Inc., 350 5th Ave., New York 1, N.Y.

Shreve, Dr. D. R. Research Math., Research Lab., Carter Oil Co., Box 801, Tulsa 2, Okla. 2709 E. 10th St., Tulsa 4.

Shui, S. S. Asst. Prof., Illinois Inst. of Tech., Chicago 16, Ill.

Shugert, S. P. Prof., Univ. of Pennsylvania, Philadelphia 4, Pa. College Hall.

Shumway, R. R. Prof. Emeritus, Univ. of Minnesota, Minneapolis 14, Minn. 3844 Thomas Ave. S., Minneapolis 10.

Shuster, C. N. Prof., New Jersey State Teachers Coll., Trenton, N.J. 2393 Pennington $\mathrm{Rd}$.

Siceloff, L. P. Prof., Columbia Univ., New York 27, N.Y.

Siedband, J. H. Instr., Illinois Inst. of Tech., Chicago 16, I1l. 4705 N. Central $\mathrm{Pk}$. Ave., Chicago 25.

Siegel, K. M. 171 W. 57th St., New York 19, N.Y.

Siegel, Roselyn A. Math., Inst. for Numerical Analysis, Univ. of California at Los Angeles, Los Angeles 24, Calif.

Sigley, Dr. D. T. Physicist, Applied Physics Lab., Johns Hopkins Univ., Silver Spring, Md.

Silver, D. N. Instr., Johns Hopkins Univ., Baltimore 18, Md. 2244 Linden Ave., Baltimore 17.

Silverman, Edward. Member, Inst. for Advanced Study, Princeton, N.J. 40 Moore St.

Silverman, L. I. Prof., Dartmouth Coll., Hanover, N.H.

Simmons, Dr. D. H. Albert Merritt Billings Hospital, 950 E. 59th St., Chicago 37, I11.

Simmons, G. F. Univ. of Chicago, Chicago 37, I11. 5424 S. University Ave., Chicago 15 .

Simmons, H. A. Asso. Prof., Northwestern Univ., Evanston, I11. 1125 Davis St.

Simon, Heinz. Prof., William Penn Coll., Oskaloosa, Iowa.

Simon, H. A. Prof., Political Science Dept., Illinois Inst. of Tech., Chicago 16, I11.

Simon, L. G. Stat., 393 7th Ave., New York 1, N.Y.

Simon, W. G. Educational Vice Pres. and Prof., Western Reserve Univ., Cleveland 6, Ohio.

Simond, Ruth G. Asst. Prof., Univ. of Vermont and State Agric. Coll., Burlington, Vt. 123 Orchard St., Franklin, N.H.

Simons, Lao G. Prof. Emeritus, Hunter Coll., New York 21, N.Y. 875 West End Ave., New York 25.

Simons, W. H. Asst. Prof., Univ. of British Columbia, Vancouver, B.C., Canada.

Simpson, Harold. Prof. Emeritus, Bedford Coll. for Women, Univ. of London, London, England. 1 Bevington Rd., Oxford, England.

Simpson, R. C. Instr., U.S. Naval Acad., Annapolis, Md.

Simpson, T. M. Dean, Grad. School, Univ. of Florida, Gainesville, Fla.

Simpson, T. W. Specialist in Applied Math., 2254 Bancroft Way, Berkeley 4, Calif. 2903 Forest Ave., Berkeley 5.

Sinclair, Annette. Asst., Univ. of Illinois, Urbana, I11. 160 Mathematics Bldg.

Sinclair, Mary Emily, Prof. Emeritus, Oberlin Coll., Oberlin, Ohio. 175 Morgan St.

Singer, David. Instr., School of Tech., City Coll., New York 31, N.Y. 1521 Ocean Ave., Brooklyn 30, N.Y.

Singer, James. Asst. Prof., Brooklyn Coll., Brooklyn 10, N.Y. 3054 Bedford Ave.

Singer, Maurice. Architect, Piqué and Singer, Architects, 4010 Eden St., New Orleans 15, La. 2603 Napoleon Ave.

Singer, Dr. P. M. Instr., Extension Div., Univ. of California, Berkeley 4, Calif. 1288 Campus Dr., Berkeley 8.

Singleton, R. R. Head, Development Dept., Aero Service Corp., 236 E. Courtland St., Philadelphia 20, Pa. 404 Burd St., Pennington, N.J.'

Singleton, Rev. T. F. Instr., Regis Coll., Denver 11, Colo.

Sips, Robert. Scientific Secretary, Belgian Chemical Union, 61 Louise Ave., Brussels, Belgium. 361 Mérode St. 
† Sisam, C. H. Prof., Colorado Coll., Colorado Springs, Colo.

Sitts, M. R. Teacher, Flint Junior Coll., Flint, Mich. 612 Bradley.

Skinner, Miriam. Columbia Univ., New York 27, N.Y. 433 E. 51st St., New York 22.

Slater, M. I. Teaching Fellow, Harvard Univ., Cambridge 38, Mass. 27 Francis Ave.

Slepian, Dr. Joseph. Asso. Dir. of Research, Westinghouse Research Labs., Westinghouse Elec. Corp., East Pittsburgh 30, Pa.

Slepin, Benjamin. Instr., Lincoln Coll. Preparatory School, Philadelphia, Pa. 422 S. 57th St., Philadelphia 43.

Sloat, F. B. Asst. Prof., Kansas State Coll. of Agric. and Applied Science, Manhattan, Kan.

Slobin, H. L. Dean Emeritus and Prof. Emeritus, Univ. of New Hampshire, Durham, N.H. 3266 6th Ave., San Diego 3, Calif.

Slook, T. H. Instr., Temple Univ., Philadelphia 22, Pa. 3610 Richmond St., Philadelphia 34.

Sloss, F. B. Prof., Westminster Coll., Fulton, Mo.

Slotnick, Dr. M. M. Supervisor, Geophysical Exploration, Humble Oil and Refining Co., Box 2180, Houston 1, Tex.

Slud, M. H. Lect., Civil Eng. Dept., Catholic Univ. of America, Washington 17, D.C.

Slutz, Dr. R. J. Design Eng., Electronic Computer Project., Inst. for Advanced Study, Princeton, N.J. 199 Nassau St.

Smail, L. L. Prof., Lehigh Univ., Bethlehem, Pa.

Smart, O. M. Eng., Naval Air Experimental Station, Navy Base, Philadelphia, Pa. Hotel Parker, 13th and Spruce Sts., Philadelphia 7.

Smiley, C. H. Dir., Ladd Observ., and Prof., Astronomy Dept., Brown Univ., Providence 12, R.I.

Smiley, Dr. Dorothy M. (Mrs. M. F.). 1716 E. Court St., Iowa City, Iowa.

Smiley, M. F. Prof., State Univ. of Iowa, Iowa City, Iowa. 1716 E. Court St.

Smith, A. H. Asso. Prof., Purdue Univ., Lafayette, Ind.

Smith, A. J. Asso. Prof., Montana School of Mines, Butte, Mont. 502 W. Granite St.

Smith, Dr. Burke. Retired. P.O. Box 314, Williams Bay, Wis.

Smith, C. B. Asso. Prof., Univ. of Florida, Gainesville, Fla. 706 N.W. 9th Ave.

Smith, Dr. C. V. L. Head, Computing Machine Sec., Office of Naval Research, Washington 25, D.C. 5006 Columbia Pike, Arlington, Va.

Smith, D. M. Prof., Georgia School of Tech., Atlanta 1, Ga.

Smith, E. R. Prof., Iowa State Coll. of Agric. and Mech. Arts, Ames, Iowa. 1113 Clark Ave.

Smith, E. S. Prof., Univ. of Cincinnati, Cincinnati 21, Ohio.

Smith, F. C. Asso. Prof., Coll. of St. Thomas, St. Paul 1, Minn.

Smith, Georgia C. (Mrs.). Asst. Prof., Spelman Coll., Atlanta, Ga.

Smith, Gertrude. Prof. Emeritus, Vassar Coll., Poughkeepsie, N.Y. Route 1, Box 191, Cumberland Center, Me.

Smith, G. W. Prof., Univ. of Kansas, Lawrence, Kan. 1730 Illinois St.

Smith, Harold E. Asst. Prof., Physics Dept., Univ. of Bridgeport, Bridgeport 5, Conn. 58 Argyle Rd., Milford, Conn.

Smith, Henry E. Asst. Prof., Dickinson Coll., Carlisle, Pa. 361 W. High St.

Smith, Helen F. Asst. Prof., Iowa State Coll. of Agric and Mech. Arts, Ames, Iowa. 113 Welch Ave.

Smith, H. L. Prof., Louisiana State Univ. and Agric. and Mech. Coll., Baton Rouge 3, La. Nicholson Hall.

Smith, H. W. Asso. Prof., Oklahoma Agric. and Mech. Coll., Stillwater, Okla.

Smith, J. C. Asst. Prof., Lafayette Coll., Easton, Pa.

Smith, Rev. J. P. Student Counsellor, Georgetown Univ., Washington 7, D.C.

Smith, P. A. Prof., Columbia Univ., New York 27, N.Y.

Smith, R. E. Asso. Prof., Coll. of William and Mary, Williamsburg, Va.

Smith, R. F. Instr., LeMoyne Coll., Memphis, Tenn.

Smith, R. G. Prof., Kansas State Teachers Coll., Pittsburg, Kan.

Smith, S. S. Asso. Prof., Univ. of Utah, Salt Lake City 1, Utah. 
Smith, T. F. 4320 Erie Ave., Cincinnati 27, Ohio.

Smith, Dr. T. L. Chief Eng., Supersonic Wind Tunnel, Ballistic Research Labs., Aberdeen Proving Ground, Md.

Smith, W. F. Asst. Prof., Univ. of Detroit, Detroit 21, Mich. P.O. Box 43.

Smith, W. M. Prof., Lafayette Coll., Easton, Pa. 2 W. College Campus.

Smith, W. N. Asst. Prof., Univ. of Wyoming, Laramie, Wyo.

Smithies, Dr. Frank. Univ. Lect., Univ. of Cambridge, Cambridge, England. 36A Bridge St.

Snapper, Ernst. Asso. Prof., Univ. of Southern California, Los Angeles 7, Calif.

Snell, L. J. Univ. of Illinois, Urbana, Ill. 1204 W. Oregon.

Snook, T. E. Retired. Hotel Newburgh, Newburgh, N.Y.

Snow, Dr. Chester. Math., National Bur. of Standards, Commerce Dept., Washington 25, D.C. 104 E. Bldg.

Snowdon, Dr. S. C. Lect., Physics Dept., Univ. of Wisconsin, Madison 6, Wis. Sterling Hall.

Snyder, A. D. Prof., Union Coll., Schenectady 8, N.Y. 1592 Union St.

Snyder, A. K. Prof., Valley Forge Military Acad. and Junior Coll., Wayne, Pa. The Kingsway.

Snyder, Virgil. Prof. Emeritus, Cornell Univ., Ithaca, N.Y. 214 University Ave.

Snyder, W. S. Asso. Prof., Univ. of Tennessee, Knoxville 16, Tenn.

Sobczyk, Andrew. Asst. Prof., Boston Univ., Boston 15, Mass. 53 Pine Ridge Rd., Arlington 74, Mass.

Soble, A. B. Control Eng. Div., General Elec. Co., 1 River Rd., Schenectady 5. N.Y.

Sohl, Dr. H. K. Asst. Prof., U. S. Naval Acad., Annapolis, Md. 523 Mt. Holly St., Baltimore 29 , Md.

Sohon, Rev. F. W. Dir., Seismological Observ., and Prof., Georgetown Univ., Washington 7, D.C.

Sokolnikoff, Elizabeth S. Asst. Prof., Univ. of Wisconsin, Madison 6, Wis. North Hall.

Sokolnikoff, I. S. Prof., Univ. of California at Los Angeles, Los Angeles 24, Calif.

Solari, Mary-Elizabeth L. (Mrs. Frank). 7 Lammas Rd., Slough, Buckinghamshire, England.

Solomon, Herbert. Senior Math. Stat., Air Intelligence Div., Strategic Vulnerability Branch, Hq., Air Forces, Washington 25, D.C.

Sopka, J. J., Teaching Fellow, Harvard Univ., Cambridge 38, Mass. 7. Harris St., Somerville, Mass.

Sorgenfrey, R. H. Asst. Prof., Univ. of California at Los Angeles, Los Angeles 24, Calif.

Sorrells, Ruth C. (Mrs. C. C.). Teacher, Highland Park Senior High School, Dallas, Tex. 619 N. Mount Clair, Dallas 11.

South, D. E. Prof., Univ. of Kentucky, Lexington 29, Ky.

Southard, T. H. Asst. Prof., Wayne Univ., Detroit 1, Mich.

Spanier, E. H. Asst. Prof., Univ. of Chicago, Chicago 37, Ill. Eckhart Hall.

Spear, Joseph. Prof., Northeastern Univ., Boston 15, Mass.

Spears, O. S. Instr., Univ. of Oklahoma, Norman, Okla. Box 2102, Boulevard Station.

Specht, E. J. Prof., Emmanuel Missionary Coll., Berrien Springs, Mich. College Station.

Specht, R. D. Asst. Prof., Univ. of Wisconsin, Madison 6, Wis. North Hall.

Spenceley, G. W. Asso. Prof., Miami Univ., Oxford, Ohio. 402 E. Church St.

Spencer, D. C. Prof., Stanford Univ., Stanford University, Calif.

Spencer, G. L. Univ. of Mich., Ann Arbor, Mich. 15 Arnold Rd., Wellesley Hills 82 , Mass.

Spencer, Dr. Vivian E. Prin. Stat., Bur. of the Census, Commerce Dept., Washington 25, D.C. Room 3073, Federal Office Bldg. No. 3.

Sperry, Pauline. Asso. Prof., Univ. of California, Berkeley 4, Calif. Box 68, Wheeler Hall.

Spicer, C. A. Prof., Western Maryland Coll., Westminster, Md. 17 Ridge Rd.

Spitzbart, Abraham. Asst. Prof., Univ. of Wisconsin in Milwaukee, Milwaukee 3 , Wis. 
Splinter, Lois L. (Mrs. O. L.). Asst. Prof., Physics Dept., Nebraska Wesleyan Univ., Lincoln, Neb. 6307 Leighton Ave., Lincoln 4.

Spohn, R. H. Instr., Lehigh Univ., Bethlehem, $\mathrm{Pa}$.

Spooner, C. C. Prof. Emeritus, Northern Michigan Coll. of Education, Marquette, Mich. 117 E. Ridge St.

Spragens, W. H. Asso. Prof., Florida State Univ., Tallahassee, Fla. 330 Hull Dr., Mabry Heights.

Sprague, A. H. Prof., Amherst Coll., Amherst, Mass. 227 S. Pleasant St.

Springer, C. E. Prof., Univ. of Oklahoma, Norman, Okla.

Springer, George. Teaching Fellow, Harvard Univ., Cambridge 38, Mass. 33 Lexington Ave.

Spurgeon, Vivian. Instr., Southwest Baptist Coll., Bolivar, Mo. 624 S. Mill.

Sreb, J. H. Physicist, Naval Research Lab., Washington 20, D.C. 1360 Peabody St. N.W., Washington 11.

Stabler, E. R. Asso. Prof., Hofstra Coll., Hempstead, N.Y.

Stachel, J. J. 203 W. 94th St., New York 25, N.Y.

Stahl, K. H. Asso. Prof., Coll. of Eng., Univ. of Colorado, Boulder, Colo.

Stahlman, W. D. Instr., Philosophy Dept., Amherst Coll., Amherst, Mass.

Staley, R. C. Prof., Univ. of North Dakota, Grand Forks, N.D. 321 Cambridge St.

Stanger, P. C. Instr., Oklahoma Agric. and Mech. Coll., Stillwater, Okla.

Stanton, Dr. R. G. Instr., Univ. of Michigan, Ann Arbor, Mich. R.F.D. 1, Lambeth, Ont., Canada.

Stanwick, C. A. Elec. Eng. 131 Rynda Rd., South Orange, N.J.

Starcher, G. W. Dean, Ohio Univ., Athens, Ohio. Northwood Dr.

Stark, Marion E. Prof., Wellesley Coll., Wellesley 81, Mass. 6 Waban St.

Stark, Dr. R. H. Math., Los Alamos Scientific Lab., Univ. of California, P.O. Box 1663, Los Alamos, N.M.

Starke, E. P. Prof., Rutgers Univ., New Brunswick, N.J.

Starr, D. W. Prof., Southern Methodist Univ., Dallas 5, Tex. 3937 Wentwood Dr.

Stauffer, J. R. K. Kingston, R.I.

Stechschulte, V. C. Prof., Xavier Univ., Cincinnati 7, Ohio.

Steed, D. V. Prof., Univ. of Southern California, Los Angeles 7, Calif.

Steele, Sister M. Philip. Asso. Prof., Rosary Coll., River Forest, Ill.

Steen, F. H. Prof., Allegheny Coll., Meadville, Pa. Route 1.

Steenrod, N. E. Asso. Prof., Princeton Univ., Princeton, N.J. Fine Hall.

Stegun, Irene A. Math., Computation Lab., National Bur. of Standards, 150 Nassau St., New York 7, N.Y. 90 Herriot St., Yonkers 2, N.Y.

Stein, Arthur. Ballistician, Ballistic Research Lab., Aberdeen Proving Ground, Md. 2 Liberty St., Aberdeen, Md.

Stein, M. L. Math., Inst. for Numericâl Analysis, National Bur. of Standards, Univ. of California at Los Angeles, Los Angeles 24, Calif. 111 N. Breed St., Los Angeles 33.

Stein, S. K. B. Lect., Columbia Univ., New York 27, N.Y. 416 Furnald Hall.

Steinberg, Robert. Instr., Univ. of California at Los Angeles, Los Angeles 24, Calif.

Steinhardt, Fritz. Lect., Columbia Univ., New York 27, N.Y. 535 W. 113th St., New York 25.

Steinhaus, Dr. H. W. Research Asst., Equitable Life Assurance Society of the U.S., 393 7th Ave., New York 1, N.Y. Elm Ridge Farm, Scarsdale, N.Y.

Stelson, H. E. Asso. Prof., Michigan State Coll., East Lansing, Mich. 300 Beal St.

Stephan, F. F. Prof., Social Stat. Dept., Princeton Univ., Princeton, N.J. 20 Nassau St.

Stephens, C. F. Prof., Morgan State Coll., Baltimore 12, Md. 1014 W. 43rd St., Baltimore 11.

Stephens, H. W. Instr., Univ. of Maryland, College Park, Md.

Stephens, Rothwell. Prof., Knox Coll., Galesburg, I11.

Stephens, R. P. Prof. Emeritus, Univ. of Georgia, Athens, Ga. 230 Woodlawn Ave.

Sternberg, Dr. Wolfgang. Math., Naval Air Magnetics Lab., Lakehurst, N.J. 
†Stetson, J. M. Prof., Coll. of William and Mary, Williamsburg, Va. 232 Jamestown Rd.

Stevenson, Guy. Prof., Univ. of Louisville, Louisville 8, Ky.

Stewart, B. M. Asso. Prof., Michigan State Coll., East Lansing, Mich. 318 Albert.

Stewart, Dr. F. M. Instr., Brown Univ., Providence 12, R.I.

Stewart, J. C. Asso. Prof., Lawrence Coll., Appleton, Wis.

Stewart, R. C. Asst. in Instruction, Yale Univ., New Haven 11, Conn. 2780 Yale Station.

Stewart, S. W. Head, Eng. Sec., Sperry Gyroscope Co., Great Neck, N.Y. 129 2d St., Garden City, N.Y.

Stibitz, Dr. G. R. Consultant. 393 S. Prospect St., Burlington, Vt.

Stinespring, (W.) F. Harvard Univ., Cambridge 38, Mass. Kirkland B-52.

Stippes, Marvin. Asst. Prof., Montana State Coll., Bozeman, Mont.

Stock, Robert. Prof., Univ. of Montreal, Montreal 26, Que., Canada. 4130 Decarie Blvd., Montreal 28.

Stoker, J. J. Prof., New York Univ., New York 3, N.Y. 66 Wayne Ave., White Plains, N.Y.

Stokes, C. N. Prof., Temple Univ., Philadelphia 22, Pa.

Stokes, Ellen C. Dean of Women and Dir. of Summer Session, New York State Coll. for Teachers, Albany 3, N.Y.

Stokes, Ruth W. Asst. Prof., Syracuse Univ., Syracuse 10, N.Y.

Stoll, R. R. Asso. Prof., Lehigh Univ., Bethlehem, Pa.

Stone, Dr. A. H. Lect., Univ. of Manchester, Manchester 13, England.

Stone, Dr. Dorothy M. (Mrs. A. H.). Univ. of Manchester, Manchester 13, England.

Stone, M. H. Prof., Univ. of Chicago, Chicago 37, I11. 313 Eckhart Hall.

Stone, R. B. Asso. Prof., Purdue Univ., Lafayette, Ind. 615 Russell St., West Lafayette, Ind.

Stone, W. M. Asst. Prof., Oregon State Coll., Corvallis, Ore.

Stoneham, R. G. Teaching Asst., Univ. of California, Berkeley 4, Calif. 5680 Oak Grove, Oakland 9, Calif.

Stoner, Irwin. Instr., Univ. of Minnesota, Minneapolis 14, Minn.

Stopher, E. C. Asst. Prof., Miami Univ., Oxford, Ohio. 106 Oberlin Ct.

Story, Helen F. Asst., Purdue Univ., Lafayette, Ind.

Stouffer, E. B. Dean, Univ. of Kansas, Lawrence, Kan. 1019 Maine St.

Stovall, W. B. 1101 Elm Ave., Sanford, Fla.

Strandberg, J. E. Instr., Riverside Coll., Riverside, Calif.

Strange, W. J. Asst. Prof., U.S. Naval Acad., Annapolis, Md.

Stratton, W. T. Prof., Kansas State Coll. of Agric. and Applied Science, Manhattan, Kan.

Straus, Dr. E. G. Instr., Univ. of California at Los Angeles, Los Angeles 24, Calif.

Straus, Louise M. (Mrs. E. G.). Univ. of California at Los Angeles, Los Angeles 24, Calif.

Strehler, A. F. Asst., Univ. of Wisconsin, Madison 6, Wis.

Strodt, Ruth C. (Mrs. Walter). Sarah Lawrence Coll., Bronxville 8, N.Y. 35 Mayhew Ave., Larchmont, N.Y.

Strodt, Walter. Asst. Prof., Columbia Univ., New York 27, N.Y. 35 Mayhew Ave., Larchmont, N.Y.

Strohl, G. R. Asst. Prof., U.S. Naval Acad., Annapolis, Md. 201 Hanover St.

Struik, D. J. Prof., Massachusetts Inst. of Tech., Cambridge 39, Mass.

Stubbe, J. S. Asst. Prof., Univ. of New Hampshire, Durham, N.H.

Studley, D. M. Dir., Organizational Research, Foundation Research, 1311 Cheyenne Blvd., Colorado Springs, Colo.

Sturley, E. A. Instr., Ällegheny Coll., Meadville, $\mathrm{Pa}$.

Suer, Loraze B. (Mrs. R. E.). Naval Hospital, Long Beach, Calif.

Suffa, Mary C. Prof., Elmira Coll., Elmira, N.Y.

Sugar, A. C. Asso. Prof., Mech. Eng. Dept., Univ. of Southern California, Los Angeles 7, Calif.

Sullivan, Sister Helen. St. Scholastica's Convent, Covington, La. 
Sullivan, Sister M. Carmel. Instr., Albertus Magnus Coll., New Haven 11, Conn. Sullivan, Mildred M. Asst. Prof., Queens Coll., Flushing, N.Y.

Sumner, D. B. Asst. Prof., Louisiana State Univ. and Agric. and Mech. Coll., Baton Rouge, La.

Sumner, Ruth G. (Mrs. J. H.). Teacher, Oakland High School, Oakland, Calif. 4281 Lakeshore, Oakland 10.

Sunde, E. D. Member of Tech. Staff, Bell Telephone Labs., Inc., 463 West St., New York 14, N.Y.

Supnick, Fred. Instr., Columbia Univ., New York 27, N.Y. 501 W. 121st St.

Suppiger, E. W. Asso. Prof., Mech. Eng. Dept., Princeton Univ., Princeton, N.J. 304 Engineering Bldg.

Sussman, Irving. Asst. Prof., Univ. of Dayton, Dayton 9, Ohio. 3251 E. Broadway, Tucson, Ariz.

Sutton, C.'S. Asst. Prof., The Citadel, Charleston, S.C.

Sutton, P. M. Columbia Univ., New York 27, N.Y. 565 Park Ave., New York 21.

Sutton, P. P. Prof. of Physics and Chemistry, State Coll. of Agric. and Eng., Univ. of North Carolina, Raleigh, N.C.

Svoboda, A. F. Asst. Prof., DePaul Univ., Chicago 1, Ill. 1815 S. Troy St., Chicago 23.

Swafford, E. G. Instr., U.S. Naval Acad., Annapolis, Md.

Swain, Dr. R. L. Instr., Ohio State Univ., Columbus 10, Ohio.

Swann, Dr. W. F. G. Dir., Bartol Research Foundation, Franklin Inst., Swarthmore, $\mathrm{Pa}$.

Swanson, L. W. Prof., Coe Coll., Cedar Rapids, Iowa; Math. Consultant, Collins Radio Co., Cedar Rapids.

Swanson, Nellie R. Prof., State Teachers Coll., Minot, N.D.

Swenson, C. R. Instr., Georgia School of Tech., Atlanta 1, Ga.

†Swift, Dr. Elijah. Retired. 415 S. Willard St., Burlington, Vt.

Swift, Dr. J. D. Instr., Univ. of California at Los Angeles, Los Angeles 24, Calif.

Swinford, L. H. Asst. Prof., Univ. of California, Berkeley 4, Calif. 420 Wheeler Hall.

Swingle, P. M. Prof., Univ. of Miami, Coral Gables 34, Fla. 215 Romano Ave.

Swope, W. G. 2809 5th Ave., Altoona, Pa.

Synge, J. L. Prof., School of Theoretical Physics, Dublin Inst. for Advanced Studies, Dublin, Ireland.

Szasz, Otto. Prof., Univ. of Cincinnati, Cincinnati 21, Ohio.

Szego, Gabor. Prof., Stanford Univ., Stanford University, Calif.

Taliaferro, Dr. R. C. Teacher, Portsmouth Priory School, Portsmouth, R.I.

Tama, Joseph. T/4, 19147363, Hq. Comdt., Hq. XXIV Corps., APO 235, San Francisco, Calif.

Tanimoto, T. T. Instr., Illinois Inst. of Tech., Chicago 16, Ill.

Tannenbaum, Benjamin. Witwatersrand Univ., Johannesburg, South Africa.

Tanzola, J. J. Chief, Pension Div., Finance Dept., New York, N.Y. 2041 Watson Ave., New York 61.

Tappan, A. Helen. Prof., Western Coll., Oxford, Ohio.

Tarski, Alfred. Prof., Univ. of California, Berkeley 4, Calif. 1001 Cragmont Ave., Berkeley 8.

Tartler, Alexander. Prof., Drexel Inst. of Tech., Philadelphia 4, Pa.

Taskin, H. K. Consulting Eng., 30 Yogurtcubasl Sokak, Kiziltoprak, Istanbul, Turkey.

Taub, A. H. Prof., Univ. of Illinois, Urbana, Ill. Inst. for Advanced Study, Princeton, N.J.

Taussky, Dr. Olga. (Mrs. John Todd). 82 Bridge Rd., East Molesey, Surrey, England.

Taylor, A. E. Asso. Prof., Univ. of California at Los Angeles, Los Angeles 24, Calif.

Taylor, Eugene. Prof., Univ. of Idaho, Moscow, Idaho.

Taylor, F. B. Instr., Manhattan Coll., New York 63, N.Y. 345 E. 193d St., New York 59. 
Taylor, H. E. Asst., Rice Inst., Houston 1, Tex. 4235 Emory St., Houston 5.

Taylor, J. H. Prof., George Washington Univ., Washington 6, D.C.

Taylor, J. J. Math., Kellex Corp., 233 Broadway, New York 7, N.Y. 785 Grange Rd., Teaneck, N.J.

Taylor, J. S. Prof., Univ, of Pittsburgh, Pittsburgh 13, Pa.

Taylor, Mildred E. Prof., Mary Baldwin Coll., Staunton, Va.

Taylor, R. L. Magdalen Coll., Oxford Univ., Oxford, England.

Taylor, William Charles. Asst. Prof., Eng. Dept., Univ. of Tennessee Junior Coll., Martin, Tenn.

Taylor, William Clare. Asst. Prof., Univ. of Cincinnati, Cincinnati 21, Ohio.

Teichmann, Theodor. Physics Dept., Princeton Univ., Princeton, N.J. Graduate College.

Temple, W. B. Asso. Prof., Louisiana Polytech. Inst., Ruston, La. Box 338, Tech. Station.

Tepping, Dr. B. J. Stat., Bur. of the Census, Commerce Dept., Washington 25, D.C.

Terracini, Alessandro. Prof., Univ. of Turin, Turin, Italy. Corso Francia 19 bis.

Terry, Ruth F. (Mrs. L. H.). Asst. Prof., Florida Southern Coll., Lakeland, Fla. 127 E. Belvedere.

Terzuoli, A. J. Instr., Polytech. Inst. of Brooklyn, Brooklyn 2, N.Y.

Thalgott, F. W. Mech. Eng., Clinton Labs., Oak Ridge, Tenn. 107 Norman Lane.

Thébault, v. M. J. Le Paradis, Tennie, France.

Theilheimer, Dr. Feodor. Math., Naval Ordnance Lab., Washington 25, D.C. 3041 M St. S.E., Washington 19.

Thielman, H. P. Prof., Iowa State Coll. of Agric. and Mech. Arts, Ames, Iowa.

Thomas, C. F. Prof. Emeritus, Case Inst. of Tech., Cleveland 6, Ohio. 3240 Scarborough Rd., Cleveland Heights 18, Ohio.

Thomas, C. J. Teaching Asst., Univ. of Chicago, Chicago 37, I11. 11471/2 E. 60th St.

Thomas, G. B. Asst. Prof., Massachusetts Inst. of Tech., Cambridge 39, Mass.

Thomas, J. M. Prof., Duke Univ., Durham, N.C. 2215 Cranford Rd.

Thomas, Sister M. Placide. Pres., Mt. St. Agnes Junior Coll., Baltimore 9, Md.

Thomas, T. Y. Prof., Indiana Univ., Bloomington, Ind. Swain Hall.

Thompson, J. E. Prof., Pratt Inst., Brooklyn 5, N.Y. 183 Steuben St.

Thompson, J. E. Instr., Univ. of Minnesota, Minneapolis 14, Minn.

Thompson, J. S. Pres., Mutual Benefit Life Ins. Co., Box 359, Newark 1, N.J.

Thompson, L. O. Instr., Univ. of Detroit, Detroit 10, Mich. Clear Creek, W.Va.

Thompson, Dr. W. R. Senior Biochemist, Div. of Labs. and Research, Health Dept., State of New York, Albany, N.Y. 1 Darroch Rd., Delmar, N.Y.

Thomsen, D. L. Asst. Prof., Haverford Coll., Haverford, Pa.

Thomson, J. F. Asso. Prof., Tulane Univ. of Louisiana, New Orleans 15, La.

Thorne, C. J. Asso. Prof., Univ. of Utah, Salt Lake City 1, Utah.

Thorne, H. H. Lect., Univ. of Sydney, Sydney, New South Wales, Australia. Fisher Library.

Thorne, P. L. Prof., New York Univ., New York 53, N.Y.

Thorpe, Alice C. (Mrs. H. A.). Instr., Univ, of Maryland, College Park, Md. 410751 st St., Bladensburg, Md.

Thrall, R. M. Asso. Prof., Univ. of Michigan, Ann Arbor, Mich. 3006 Angell Hall.

Thron, W. J. Asst. Prof., Washington Univ., St. Louis 5, Mo.

Thullen, Dr. Peter. Actuary, Colombian Ins. Co., Bogota, Colombia.

Thurston, H. S. Prof., Univ. of Alabama, University, Ala. P.O. Box 1453.

Tiller, G. L. Asst. Prof., Utica Coll., Syracuse Univ., Utica, N.Y.

Tilley, Arthur. Asso. Prof., New York Univ., New York 3, N.Y.

Timoshenko, S. P. Prof. Emeritus, Stanford Univ., Stanford University, Calif. 536 W. Crescent Dr., Palo Alto, Calif.

Tingey, v. H. Prof., Utah State Agric. Coll., Logan, Utah. College Hill.

Tinnappel, H. E. Asst., Ohio State Univ., Columbus 10, Ohio. 4212 Fisher Rd., Columbus 4.

Tintner, Gerhard. Prof., Iowa State Coll. of Agric. and Mech. Arts, Ames, Iowa. Research Asso., Applied Economics Dept., Cambridge Univ., Cambridge, England. 
Titt, E. W. Asso. Prof., Univ. of Texas, Austin 12, Tex. 2206 Newfield Lane, Austin 21.

Titus, Dr. C. J. Instr., Syracuse Univ., Syracuse 10, N.Y.

Todd, John. 82 Bridge Rd., East Molesey, Surrey, England.

Togliatti, E. G. Prof., Univ. of Genoa, Genoa, Italy. 11-4 Tommaso Invrea Way.

de Toledo Piza, A. P. Tech. Consultant, Stat. Dept., São Paulo, Brazil. 1123 Ministro Godoi St.

Tolle, L. F. Prof., St. John's Univ., Brooklyn 6, N.Y. 240-23 141st Ave., Jamaica 10, N.Y.

Tolsted, Elmer. Asst. Prof., Pomona Coll., Claremont, Calif.

Tomber, M. L. Instr., Univ. of Pennsylvania, Philadelphia 4, Pa. P.O. Box 53, Central Islip, N.Y.

Tompkins, Dr. C. B. Tech. Advisor, Eng. Research Associates, 507 18th St. S., Arlington, Va.

Tong, Dr. Hing. Instr., Barnard Coll., Columbia Univ., New York 27, N.Y. John Jay Hall.

Toops, H. A. Prof., Ohio State Univ., Columbus 10, Ohio. 1430 Cambridge Blvd., Columbus 8.

Topp, C. W. Asst. Prof., Fenn Coll., Cleveland 15, Ohio. 1524 Compton Rd., Cleveland Heights 18, Ohio.

Toralballa, Dr. L. V. Instr., Fordham Univ., New York 58, N.Y. 2706 Valentine Ave.

Tornheim, Dr. Leonard. Instr., Univ. of Michigan, Ann Arbor, Mich. 211 Pine Ridge.

Torrance, C. C. Asso. Prof., U.S. Naval Postgrad. School, Annapolis, Md. R.F.D. 3.

Torrance, Dr. Esther M. (Mrs. C. C.). R.F.D. 3, Annapolis, Md.

Torrey, Marian M. Prof., Goucher Coll., Baltimore 18, Md.

Townsend, E. J. Prof. Emeritus, Univ. of Illinois, Urbana, I11. 510 E. John St., Champaign, I11.

Towson, L. R. Asso. Prof., North Georgia Coll., Dahlonega, Ga.

Trabant, Dr. E. A. Instr., Purdue Univ., Lafayette, Ind.

Tracey, J. I. Asso. Prof., Yale Univ., New Haven 11, Conn. 84 McKinley Ave., New Haven 15.

Transue, Dr. W. R. Asst., Inst. for Advanced Study, Princeton, N.J.

Trapani, B. J. Instr., Keystone Junior Coll., La Plume, Pa. Factoryville, Pa.

Trauring, Mitchell. Johns Hopkins Univ., Baltimore 18, Md. 3301 St. Paul St.

Treiber, H. I. Math., Watson Labs., Red Bank, N.J. 74 White St., Long Branch, N.J.

Trejo, C. A. Asst. Prof., Univ. of LaPlata, LaPlata, Argentina. 407 46th St.

Trejos-Fernández, J. J. Prof., Univ. of Costa Rica, San José, Costa Rica. Ap. 1313.

Tremblay, C. J. Instr., Bard Coll., Annandale-on-Hudson, N.Y.

Trennt, Evelyn L. Instr., Springfield Junior Coll., Springfield, Il1.

Treon, Marion. Instr., Ohio Univ., Athens, Ohio.

Treuenfels, P. M. Inst. for Math. and Mech., New York Univ., New York 3, N.Y. 140 Bay Ridge Pkwy., Brooklyn 9, N.Y.

Trevifio García, José. 1302 Hidalgo Pt., Monterrey, N.L., Mexico.

Tricomi, F. G. Prof., Univ. of Turin, Turin, Italy. Asso., California Inst. of Tech., Pasadena 4, Calif.

Trifan, Dr. Deonisie. Instr., Case Inst. of Tech., Cleveland 6, Ohio, 3872 W. 162d St., Cleveland 11.

Trimble, H. C. Asso. Prof., Florida State Univ., Tallahassee, Fla. Box 1013.

Tripp, M. O. Prof. Emeritus, Wittenberg Coll., Springfield, Ohio. 218 West Cecil, Springfield 30 .

Tripp, Dr. R. H. Head of Research Dept., Grumman Aircraft Eng. Corp., Bethpage, N.Y.

Trjitzinsky, W. J. Prof., Univ. of Illinois, Urbana, I1l.

Trombley, E. F, Asst., Univ. of Chicago, Chicago 37, I11. Box 47, Eckhart Hall.

Trott, G. R. Prof., Univ. of Mississippi, University, Miss. P.O. Box 523.

Truell, Rohn. Asso. Prof., Physics Dept., Brown Univ., Providence 12, R.I. 27 Brown St., Providence 6. 
Truesdell, Dr. C. A. Consultant, Applied Math. Sec., Mech. Div, Naval Research Lab., Washington 20, D.C.; Asso. Prof., Univ. of Maryland, College Park, Md.

Trump, P. L. Dean of Men and Asso. Dir. for Student Personnel Service, Univ. of Wisconsin, Madison 6, Wis. 2228 Eton Ridge, Madison 5.

Tryon, G. M. V. 105 W. Shiawassee Ave., Fenton, Mich.

Tsien, H. S. Prof. of Aerodynamics, Massachusetts Inst. of Tech., Cambridge 39 , Mass.

Tuan, H. F. Prof., Tsing Hua Univ., Peiping, China.

Tucker, A. W. Prof., Princeton Univ., Princeton, N.J. Fine Hall.

Tucker, C. B. Asso. Prof., Kansas State Teachers Coll., Emporia, Kan.

Tuckerman, Dr. Bryant. Instr., Cornell Univ., Ithaca, N.Y. White Hall.

Tukey, J. W. Asso. Prof., Princeton Univ., Princeton, N.J.; Member of Tech. Staff, Bell Telephone Labs., Murray Hill, N.J. Box 708, Fine Hall, Princeton.

Tuller, Dr. Annita. Instr., Hunter Coll., New York 21, N.Y. 139-62 Pershing Crescent, Jamaica 2, N.Y.

Tulloch, L. H. Asso. Prof., Southwest Texas State Coll., San Marcos, Tex.

Tully, G. A. Supervisor, Tabulating Unit, Comptrollers Dept., State Capitol Bldg., Little Rock, Ark. 4812 W. 25th St.

Turán, Dr. Paul. Izabella u 41 III rm 23, Budapest VI, Hungary.

Turner, Bird M. Prof. Emeritus, West Virginia Univ., Morgantown, W.Va. $61 \mathrm{Wils}$ on Ave.

Turner, Dr. J. S. Master, Wycliffe Coll., Stonehouse, Gloucestershire, England.

Turner, Lona L. Univ. of Chicago, Chicago 37, I11. 5709 Ellis Ave.

Turner, Dr. Mary H. (Mrs. M. J.). 174 Plymouth St., Stratford, Conn.

Turner, V. D. Univ. of Illinois, Urbana, Ill. 503 S. Wright St., Champaign, Ill.

Turquette, A. R. Asso. Prof., Philosophy Dept., Univ. of Illinois, Urbana, Ill. 322 Gregory Hall.

Turrin, Gino. Prof., Univ. of Cuyo, San Luis, Argentina. 2675 José C. Paz Ave., Olivos, F.C.C.A., Argentina.

Turrittin, H. L. Asso. Prof., Univ. of Minnesota, Minneapolis 14, Minn. 4046 Beard Ave. S., Minneapolis 10.

Tye, Bradford. Asst. Prof., Bethany Coll., Bethany, W:Va. Box 154.

Tyler, E. F. Research Eng., Douglas Aircraft Co., Inc., E1 Segundo, Calif. 3337 Stocker St., Los Angeles 43, Calif.

Uhl, H. R. Fellow, Tulane Univ. of Louisiana, New Orleans 15, La. 2300 Moss St., New Orleans 19.

Uhler, H. S. Prof. Emeritus, Yale Univ., New Haven 11, Conn. 206 Spring St., Meriden, Conn.

Uhrich, G. E. Asso., Univ. of Washington, Seattle 5, Wash. 120 E. 52d St.

Ulam, Dr. S. M. 1149-B 23d St., Los Alamos, N.M.

Ullman, J. L. Stanford Univ., Stanford University, Calif.

Ulmer, Gilbert. Asst. Dean and Asso. Prof., Univ. of Kansas, Lawrence, Kan.

Ulrich, F. E. Asso. Prof., Rice Inst., Houston 1, Tex.

Uluçay, Dr. Cengiz. Nisantasi, Tesvikiye caddesi, Inayet Apart. No. 3, Istanbul, Turkey.

Umberger, E. H. Instr., Pennsylvania State Coll., State College, Pa. 511 E. Foster Ave.

Updike, G. Z. Instr., City Coll., New York 31, N.Y.

Upton, C. B. Prof. Emeritus, Teachers Coll., Columbia Univ., New York 27, N.Y.

Urner, Dr. S. E. Instr., Los Angeles City Coll., Los Angeles 27, Calif. 5141 Fulton Ave., Sherman Oaks, Calif.

Utz, Dr. W. R. Instr., Univ. of Michigan, Ann Arbor, Mich. 3020 Angell Hall.

Vaage, E. F. Eng., Bell Telephone Labs., Inc., 463 West St., New York 14, N.Y.

Valentine, F. A. Asst. Prof., Univ. of California at Los Angeles, Los Angeles 24, Calif.

Valiron, Georges. Prof., Univ. of Paris, Paris, France. 95 Jourdan Blvd., Paris 14. 
Vallarta, Dr. M. S. General Dir., National Polytech. Inst., Mexico, D.F., Mexico. 100 Monterrey St.

Van Andel, J. R. Asst., Moore School of Elec. Eng., Univ. of Pennsylvania, Philadelphia 4, Pa. 3418 Walnut St.

Van Bergen, Dr. F. E. Instr., Royal Athenaeum of St. Nicolas, St. NicolasWaas, Belgium. 15 Spoorweglaan.

Van Buskirk, H. C. Prof. Emeritus, California Inst. of Tech., Pasadena 4, Calif. 390 S. Holliston Ave., Pasadena 5.

Vance, E. P. Asst. Prof., Oberlin Coll., Oberlin, Ohio.

Vanderburg, Bethuhne. Asst., Univ. of Chicago, Chicago 37, I11. 919 E. 56th St.

Vanderslice, J. L. Asso. Prof., Univ. of Maryland, College Park, Md.

Vandiver, H. S. Prof., Univ. of Texas, Austin 12, Tex.

Van Dyke, Louisa A. M. Prof., Flora Macdonald Coll., Red Springs, N.C.

Van Engen, Henry. Asso. Prof., Iowa State Teachers Coll., Cedar Falls, Iowa.

† Van Orstrand, C. E. Retired. Route 2, Manito, I1l.

Vansant, Helen E. Asso. Prof., Beaver Coll., Jenkintown, $\mathrm{Pa}$.

Van Schaack, G. B. Asso. Prof., Washington Univ., St. Louis 5, Mo. Missouri Botanical Garden, 2315 Tower Grove Ave., St. Louis 10.

Van Tuyl, Dr. A. H. Math., Naval Ordnance Lab., White Oak, Silver Spring 19, Md. 1460 Harvard St. N.W., Washington 9, D.C.

Van Vleck, J. H. Prof., Physics Dept., Harvard Univ., Cambridge 38, Mass. Lyman Laboratory of Physics.

Varineau, V. J. Asst. Prof., Univ. of Wyoming, Laramie, Wyo.

Varney, R. N. Prof., Physics Dept., Washington Univ., St. Louis 5, Mo.

Vass, Dr. J. I. Retired. 5141 N. Santa Monica Blvd., Milwaukee 11, Wis.

Vassallo, Walter. Prof., Physics Dept., Univ. of Portland, Portland 3, Ore.

Vassiliou, Philon, Prof., National Univ. of Eng. Science, Patissia, Athens, Greece. 30 Keas St.

Vaudreuil, Sister M. Felice. Prof., Mt. Mary Coll., Milwaukee 13, Wis.

Vaughan, H. E. Asst. Prof., Univ. of Illinois, Urbana, Ill. 907 S. Vine St.

Vause, R. Z. Instr., Clemson Coll., Clemson, S.C. P.O. Box 1249.

Vazsonyi, Dr. And̈rew. Acting Head, Guided Missile Div., Naval Ordnance Test Station, Pasadena, Calif. 42 S. Grand Ave.

† Veblen, Oswald. Prof., Inst. for Advanced Study, Princeton, N.J. 58 Battle Rd.

Velesz, Rev. D. G. Instr., Quincy Coll., Quincy, Il1.

Ventriglia, A. E. Instr., Manhattan Coll., New York 63, N.Y. 317 Hayward Ave., Mt. Vernon, N.Y.

Vermes, Dr. Paul. Lect., Birkbeck Coll., Univ. of London, London, England. 30 Greencroft Gardens, London N.W. 6.

Vest, M. L. Asst. Prof., West Virginia Univ., Morgantown, W.Va.

Vezeau, W. A. Asst. Prof., St. Louis Univ., St. Louis 3, Mo.

Vickery, C. W. Asso. Prof., Louisiana State Univ. and Agric. and Mech. Coll., Baton Rouge 3, La. Box 6890.

Vigder, J. S. Asst. Prof., Univ. of Saskatchewan, Saskatoon, Sask., Canada.

† Vijayaraghavan, Thirukkannapuram. Prof., Andhra Univ., Waltair, India.

Vincensini, Paul. Prof., Univ. of Besançon, Besançon, France.

Vinograde, Bernard. Asso. Prof., Iowa State Coll. of Agric. and Mech. Arts, Ames, Iowa. Beardshear Hall.

Vitale, R. L. Asst. Elec. Eng., Board of Transportation of the City of New York, 250 Hudson St., New York 13, N.Y. 366 Ave. T, Brooklyn 23, N.Y.

Vogel, Bess B. (Mrs. A. M.). Instr., Adelphi Coll., Garden City, N.Y. 230 E. 87 th St., New York 28, N.Y.

Vogel, Barbara R. (Mrs. R. C.). Illinois Inst. of Tech., Chicago 16, I11. 4603 Lake Park Ave., Chicago 15.

Votaw, Dr. D. F. Instr., Yale Univ., New Haven 11, Conn. 210 Leet Oliver Memorial Hall.

Vrooman, S. I. Instr., Rensselaer Polytech. Inst., Troy, N.Y. 180 8th St.

Waddell, M. C. Johns Hopkins Univ., Baltimore 18, Md.

Waddell, Mary E. G. Univ. of Toronto, Toronto 5, Ont., Canada. 72 Madison Ave., Toronto. 
Wade, L. I. Prof., Louisiana State Univ. and Agric. and Mech. Coll., Baton Rouge 3, La.

Wade, T. L. Prof., Florida State Univ., Tallahassee, Fla. Box 1106.

van der Waerden, B. L. Prof., Univ. of Amsterdam, Amsterdam O, Netherlands. Verlengde Engweg 10, Laren N, Netherlands.

Wagner, R. D. Dir., Marinette Extension Center, and Asst. Prof., Univ. of Wisconsin, Marinette, Wis.

Wagner, R. W. Asst. Prof., Oberlin Coll., Oberlin, Ohio.

Wagner, Dr. T. C. G. Senior Eng., Davies Labs., Inc., College Park, Md.; Asso. Prof., Elec. Eng. Dept., Univ. of Maryland. 4995 Newport Ave., Washington 16, D.C.

Wakerling, Dr. R. K. Head, Tech. Information Div., Radiation Lab., Univ. of California, Berkeley 4, Calif. 2834 Derby St., Berkeley 5.

Wald, Abraham. Prof., Math Stat. Dept., Columbia Univ., New York 27, N.Y.

Walder, O. E. Prof., South Dakota State Coll., Brookings, S.D. College Station.

Walker, A. G. Prof., Univ. of Sheffield, Sheffield 10, England.

Walker, B. M. Pres. Emeritus, Mississippi State Coll., State College, Miss. Starkville, Miss.

Walker, G. L. Asst. Prof., Purdue Univ., Lafayette, Ind.

Walker, Rev. G. W. Pastor, Walden Presbyterian Church, Buffalo, N.Y. 2065 Bailey Ave., Buffalo 11.

Walker, Helen M. Prof., Teachers Coll., Columbia Univ., New York 27, N.Y.

Walker, R. J. Prof., Cornell Univ., Ithaca, N.Y. White Hall.

Walkley, S. E. Univ. of Illinois, Urbana, Ill. 160 Mathematics Bldg.

Wall, H. S. Prof., Univ. of Texas, Austin 12, Tex. 11 Waggener Hall.

Wallace, A. D. Prof., Tulane Univ. of Louisiana, New Orleans 15, La. 306 Gibson Hall.

Wallach, Dr. Sylvan. Instr., Johns Hopkins Univ., Baltimore 18, Md. 1348 Pentwood Rd., Baltimore 12.

Wallick, E. E. Teacher, Lakewood Senior High School, Lakewood, N.J. 426 3d St.

Wallman, Henry. Asso. Prof., Massachusetts Inst. of Tech., Cambridge 39, Mass. Chalmers Inst. of Tech., Gothenburg, Sweden.

Walmsley, Charles. Asso. Prof., Dalhousie Univ., Halifax, N.S., Canada. 6 Studley Ave.

Walsh, J. L. Prof., Harvard Univ., Cambridge 38, Mass. 474 Widener Library.

Walter, R. M. Asso. Prof., New Jersey Coll. for Women, Rutgers Univ., New Brunswick, N.J.

Walters, Eleanor B. Asso Prof., Delta State Teachers Coll., Cleveland, Miss.

Walton, Dr. Jean B. Instr., Univ. of Pennsylvania, Philadelphia 4, Pa. R.F.D. 3, Media, Pa.

Walton, L. F. Asst. Prof., Santa Barbara Coll., Univ. of California, Santa Barbara, Calif.

Walton, Sister M. Bertrand. Prof., Marywood Coll., Scranton 9, Pa.

Walton, T. O. Prof., Kalamazoo Coll., Kalamazoo 49, Mich.

Wang, C. T. Asso. Prof., Aeronautics Dept., New York Univ., New York 53, N.Y.

Wang, C. Y. Univ. of Minnesota, Minneapolis 14, Minn. 2829 16th Ave. S.

Ward, J. A. Prof., Univ. of Georgia, Athens, Ga.

Ward, Dr. L. E. Math., Naval Ordnance Test Station, China Lake, Calif. 206-B E1lis St.

Ward, Morgan. Prof., California Inst. of Tech., Pasadena 4, Calif.

Wardwell, J. F. Asso. Prof., Colgate Univ., Hamilton, N.Y.

Ware, Mamie S. (Mrs.). Instr., Clark Coll., Atlanta, Ga. 719 W. Fair St. N.W.

Warnock, W. G. Prof., Rensselaer Polytech. Inst., Troy, N.Y. Melrose, N.Y.

Warren, J. E. Supervisor, Government Sec., Labs. Div., Teleregister Corp., 157 Chambers St., New York 7, N.Y. 8 Ruby P1., Newark 4, N.J.

Warschawski, S. E. Prof., Univ. of Minnesota, Minneapolis 14, Minn.

Washburne, A. C. Actuary Emeritus, Berkshire Life Ins. Co., Pittsfield, Mass.

Washnitzer, Gerard. Instr., Brooklyn Coll., Brooklyn 10, N.Y. 1030 Carroll St., Brooklyn 25. 
Wasow, W. R. Asst. Prof., Swarthmore Coll., Swarthmore, Pa. 915 Harvard Ave. Watanabe, Dr. Kenichi. Physicist, Optics Div., Naval Research Lab., Washington 20, D.C. 4318 3d St. S.E.

Waterman, D. R. Instr., Johns Hopkins Univ., Baltimore 18, Md.

Watkeys, C. W. Prof., Univ. of Rochester, Rochester 3, N.Y. 287 Dartmouth St., Rochester 7.

Watson, Marjorie. Instr., Univ. of Tennessee, Knoxville 16, Tenn.

Wayne, Alan. Teacher, Brooklyn High School of Automotive Trades, Brooklyn 6, N.Y. 141-21 78th Rd., Flushing, N.Y.

Wear, L. E. Asso. Prof., California Inst. of Tech., Pasadena 4, Calif. 2247 Lambert Dr., Pasadena 8.

Weatherburn, C. E. Prof., Univ. of Western Australia, Perth, Western Australia, Australia.

Weaver, Theodore. Asst. Prof., Michigan State Normal Coll., Ypsilanti, Mich. Box 286.

Weaver, Dr. Warren. Dir., Div. of Natural Sciences, Rockefeller Foundation, 49 W. 49th St., New York 20, N.Y.

Webb, D. L. Asso. Prof., Univ. of Arizona, Tucson, Ariz.

Webber, G. C. Prof., Univ. of Delaware, Newark, Del.

Webber, W. J. Asso. Prof., Univ. of Toronto, Toronto 5, Ont., Canada.

Weber, Ernst. Prof., Elec. Eng. Dept., Polytech. Inst. of Brooklyn, Brooklyn 2, N.Y.

Weber, Maria A. Instr., Goucher Coll., Baltimore 18, Md.

Weber, W. W. Asso. Prof., Univ. of South Carolina, Columbia 19, S.C. 1516 Columbia College Dr.

Webster, J. B. Columbia Univ., New York 27, N.Y. 81 Columbia Heights, Brooklyn 2, N.Y.

Webster, M. S. Asso. Prof., Purdue Univ., Lafayette, Ind.

Wechsler, M. T. Univ. of Michigan, Ann Arbor, Mich. 934 Dewey Ave.

Wehausen, Dr. J. V. Math., David Taylor Model Basin, Navy Dept., Washington 7, D.C. 126 Winchester Way, Falls Church, Va.

Weida, F. M. Prof., Stat. Dept., George Washington Univ., Washington 6, D.C.

Weil, André. Prof., Univ. of Chicago, Chicago 37, I11. Eckhart Hall.

Weil, Herschel. Asso., Brown Univ., Providence 12, R.I. 92 Lyndon Rd., Cranston 5 , R.I.

Weingarten, Harry. Tutor, City Coll., New York 31, N.Y.; Instr., Div of General Education, New York Univ., New York. 347 W. 55th St., New York 19.

Weinstein, Alexander. Prof., Univ. of Maryland, College Park, Md.; Senior Research Asso., Naval Ordnance Labs., White Oak, Silver Spring 19, Md. 10-B N. Manor Circle, Tacoma Park, Md.

Weinstein, Alma K. (Mrs. D. H.). 1904 Branard St., Houston 6, Tex.

Weisbecker, Frances. Instr., Milwaukee-Downer Coll., Milwaukee 11, Wis. 830 E. Mason St., Milwaukee 2.

Weisner, Louis. Asso. Prof., Hunter Coll., New York 21, N.Y. 1188 Sheridan Ave., New York 56.

Weiss, Marie J. Prof., Newcomb Memorial Coll., Tulane Univ. of Louisiana, New Orleans 18, La.

Welch, Brother B. A. Prof., Manhattan Coll., New York 63, N.Y.

Welch, F. P. Prof., Washington and Lee Univ., Lexington, Va. 303 S. Jefferson St.

Welker, Dr. E. L. Asso., Bur. of Medical Economic Research, American Medical Assn., 535 N. Dearborn, Chicago 10, I11. 4901 Montgomery Ave., Downers Grove, I11.

Wells, C. P. Asso. Prof., Michigan State Coll., East Lansing, Mich. Brown Univ., Providence 12, R.I.

Wells, Mary Evelyn. Prof. Emeritus, Vassar Coll., Poughkeepsie, N.Y.

Wells, N. W. Instr., Agric. and Mech. Coll. of Texas, College Station, Tex. 1928 Ave. N r 2 , Galveston, Tex.

Wells, V. H. Prof., Williams Coll., Williamstown, Mass. 3 Chapin Ct.

Welmers, Dr. E. T. Group Leader, Dynamic Analysis, Bell Aircraft Corp., Buffalo, N.Y. 165 Fayette Ave., Kenmore 17, N.Y. 
Welmers, Ina W. (Mrs. E. T.). Instr., Univ. of Buffalo, Buffalo 14, N.Y. 165 Fayette Ave., Kenmore 17, N.Y.

Welsh, C. A. Asso. Prof., Economics Dept., American Univ., Washington 16, D.C. 203 N. Trenton St., Arlington, Va.

Wend, D. V. V. Univ. of Michigan, Ann Arbor, Mich. 1004 Vaughn St.

Wendel, Dr. J. G. Instr., Yale Univ., New Haven 11, Conn.

Wente, Irene L. Asso. Prof., South Dakota State Coll., Brookings, S.D. State College Station.

Wernick, Dr. William. Teacher, Julia Richman High School, New York 21, N.Y. 2515 Davidson Ave., New York 63.

Wertheimer, Albert. Eng., Bur. of Ordnance, Navy Dept., Washington 25, D.C.

Wescott, M. E. Asst. Prof., Northwestern Univ., Evanston, I11. 2011 Beechwood Dr., Wilmette, Ill.

West, C. T. Asst. Prof., Mech. Dept., Ohio State Univ., Columbus 10, Ohio.

Westerfield, Dr. E. C. Physicist, Navy Electronics Lab., San Diego 52, Calif. Box 206, Point Loma, San Diego 6.

† Western, Dr. A. E. Windwhistle, Grayshott, Hindhead, Surrey, England.

Western, D. W. Asso. Prof., Franklin and Marshall Coll., Lancaster, Pa.

Westfall, W. D. A. Prof., Univ. of Missouri, Columbia, Mo. 11 S. Glenwood.

de Wet, Dr. J. S. Fellow, Balliol Coll., Oxford Univ., Oxford, England.

Wetherell, Elizabeth H. Instr., Syracuse Univ., Syracuse 10, N.Y. 415 Sedgwick Dr., Syracuse 3.

Wetzel, R. A. Retired. 218 Tecumseh Ave., Mt. Vernon, N.Y.

Weyl, Dr. F. J. Math., Office of Naval Research, Navy Dept., Washington 25, D.C. Route 3, Fairfax, Va.

Weyl, Hermann. Prof., Inst. for Advanced Study, Princeton, N.J.

Whaples, George. Asso. Prof., Indiana Univ., Bloomington, Ind. Swain Hall.

Wheeler, A. H. Retired. 44 Beverly Rd., Worcester 5, Mass.

Wheeler, Dr. C. H. Treas., Univ. of Richmond, University of Richmond, Va.

Wheeler, J. J. Asso. Prof. Emeritus, Univ. of Kansas, Lawrence, Kan. 1024 Alabama St.

Whelan, A. Marie. Asst. Prof., Hunter Coll., New York 21, N.Y.

White, E. F. Asst. Prof., Univ. of Cincinnati, Cincinnati 21, Ohio.

White, J. H. Asst. Prof., U.S. Naval Acad., Annapolis, Md. Riva, Md.

White, L. R. 3182 Westover Dr. S.E., Washington 20, D.C.

White, Dr. Marion B. Retired. 1949 Woodlyn Rd., Pasadena 7, Calif.

White, M. E. 105 W. 16th St., New York 11, N.Y.

White, P. A. Asso. Prof., Univ. of Southern California, Los Angeles 7, Calif. 3821 S. Degnan Blvd.

White, R. L. Univ. of California at Los Angeles, Los Angeles 24, Calif. 2122 W. Washington Blvd., Los Angeles 7.

White, T. J. Asst., Rice Inst., Houston 1, Tex. $2509 \mathrm{McClendon}$, Houston 5.

Whitehead, G. W. Asso. Prof., Brown Univ., Providence 12, R.I.

Whitehead, J. H. C. Prof., Oxford Univ., Oxford, England. 22 Charlbury Rd.

Whitehead, Dr. Kathleen B. (Mrs. G. W.). Instr., Brown Univ., Providence 12, R.I.

Whiteman, A. L. Asst. Prof., Univ. of Southern California, Los Angeles 7, Calif.

Whitford, D. E. Asst. Prof., Polytech. Inst. of Brooklyn, Brooklyn 2, N.Y.

Whitman, E. A. Asso. Prof., Carnegie Inst. of Tech., Pittsburgh 13, Pa.

Whitman, Dr. P. M. Math., Applied Physics Lab., Johns Hopkins Univ., Silver Spring, Md.

Whitmore, R. M. Asst. Prof., Southwestern Univ., Georgetown, Tex. Box 15, Southwestern University Station.

Whitmore, Dr. W. F. Operations Analyst, Operations Evaluation Group, (CNO), Navy Dept., Washington 25, D.C. 6415 MacArthur Blvd., Washington 16.

Whitney, Anne M. Univ. of Pennsylvania, Philadelphia 4, Pa. 4720 Cedar Ave., Philadelphia 43.

Whitney, D. R. Asst. Prof., Ohio State Univ., Columbus 10, Ohio. 39 Tibet Rd., Columbus 2.

Whitney, Hassler. Prof., Harvard Univ., Cambridge 38, Mass.

† Whittaker, Sir Edmund T. Retired. 48 George Sq., Edinburgh 8, Scotland. 
Whyburn, G. T. Prof., Univ. of Virginia, Charlottesville, Va.

Whyburn, Lucille S. (Mrs. G. T.). Acting Asst. Prof., Univ. of Virginia, Charlottesville, Va. 133 Bollingwood Rd.

Whyburn, W. M. Prof., Univ. of North Carolina, Chapel Hill, N.C.

†Widder, D. V. Prof., Harvard Univ., Cambridge 38, Mass. 12 Hunt Hall.

Widder, Dr. Vera A. (Mrs. D. V.). Instr., Cambridge Junior Coll., Cambridge 38, Mass. 30 Gould Rd., Arlington 74, Mass.

Widmer, W. J. Instr., Civil Eng. Dept., Univ. of Connecticut, Storrs, Conn.

Wiegmann, Dr. N. A. Instr., Univ. of Michigan, Ann Arbor, Mich.

Wiener, Norbert, Prof., Massachusetts Inst. of Tech., Cambridge 39, Mass. 53 Cedar Rd., Belmont 78, Mass.

Wiggin, Evelyn P. Prof., Randolph-Macon Woman's Coll., Lynchburg, Va.

Wightman, A. S. Princeton Univ., Princeton, N.J. Physics Dept.

Wigner, E. P. Prof., Physics Dept., Princeton Univ., Princeton, N.J. Fine Hall.

Wilansky, Albert. Asst. Prof., Lehigh Univ., Bethlehem, Pa.

Wilcox, L. R. Asso. Prof., Illinois Inst. of Tech., Chicago 16, Ill.

Wilcox, Mary E. Asst. Prof., Southwestern Univ., Georgetown, Tex. 1202 E. 12th St.

Wilczewski, Rev. Joseph. Prof., Marquette Univ., Milwaukee 3, Wis.

Wilder, C. E. Prof., Dartmouth Coll., Hanover, N.H. 5 Dana Rd.

Wilder, R. L. Prof., Univ. of Michigan, Ann Arbor, Mich. 1617 Cambridge Rd.

Wildermuth, R. B. Prof. Capital Univ., Columbus 9, Ohio. 2285 E. Mound St.

Wiley, F. B. Prof., Denison Univ., Granville, Ohio.

Wiley, R. B. Research Asst., Owens-Corning Fiberglas Corp., Newark, Ohio. 282 Elmwood Ave.

Wilkins, Dr. J. E. Math., American Optical Co., Box A, Buffalo 15, N.Y. 557 Stockbridge Ave.

Wilks, S. S. Prof., Princeton Univ., Princeton, N.J. Fine Hall.

Willerding, Dr. Margaret F. Instr., Harris Teachers Coll., St. Louis 4, Mo. 5225 Walsh, St. Louis 9.

Williams, A. R. Asst. Prof., Univ. of California, Berkeley 4, Calif. 455 Wheeler Hall.

Williams, Dr. Christine S. Instr., Cornell Univ., Ithaca, N.Y. White Hall.

Williams, C. W. Asst. Prof., Washington and Lee Univ., Lexington, Va.

Williams, Dr. Emily C. (Mrs. W. H.). Care of Central Hanover Bank and Trust Co., 5th Ave. and 60th St., New York 22, N.Y.

Williams, Dr. H. B. Consultant, Cambridge Instrument Co., 3732 Grand Central Terminal Bldg., New York 17, N.Y.

Williams, J. B. Univ. of New Hampshire, Durham, N.H. 7 Spring St., Exeter, N.H.

Williams, J. D. 15070 Corona Del Mar, Pacific Palisades, Calif.

Williams, J. F. Univ. of Tennessee, Knoxville 16, Tenn. Schubert Dr., Route 2, Fountain City, Tenn.

Williams, J. N. Asst., Yale Univ., New Haven 11, Conn. 236 Whitney Ave.

Williams, K. P. Prof., Indiana Univ., Bloomington, Ind.

Williams, L. B. Asst. Prof., Reed Coll., Portland 2, Ore.

Williams, Roger. Asst. Editor, Chemical Eng., McGraw-Hill Publishing Co., 330 W. 42 d St., New York 18, N.Y. 9 Sniffen Ct., New York 19.

Williams, W. J. Prof., Baylor Univ., Waco, Tex.

Williams, W. L. Prof., Univ. of South Carolina, Columbia 19, S.C.

Williams, W. L. G. Prof., McGill Univ., Montreal, Que., Canada. Engineering Bldg.

Williamson, C. O. Prof., Coll. of Wooster, Wooster, Ohio. 1141 Beall Ave.

Williamson, John. Prof., Queens Coll., Flushing, N.Y.

Wilson, Dr. A. G. Fellow, Astro-Physics Dept., California Inst. of Tech., Pasadena 4, Calif. $1759 \mathrm{La} \mathrm{Paz}$ Rd., Altadena, Calif.

Wilson, A. H. Prof. Emeritus, Haverford Coll., Haverford, Pa.

Wilson, A. L. Instr., Univ. of Vermont and State Agric. Coll., Burlington, Vt. 400 Hamilton Ave., Ottawa, Ont., Canada.

Wilson, E. B. Prof. Emeritus, Harvard Univ., Cambridge 38, Mass.; Consultant, Office of Naval Research, Boston, Mass. School of Public Health, Harvard Univ., Boston 15. 
Wilson, Dr. Elizabeth W. Editor, National Physicians' Com., 75 E. Wacker Dr., Chicago 1, Ill. 1 Waterhouse St., Cambridge 38, Mass.

Wilson, L. T. Prof., U.S. Naval Acad., Annapolis, Md. 20 Thompson St.

Wilson, R. L. Asst. Prof., Univ. of Tennessee, Knoxville 16, Tenn.

Wilson, Wilfrid. Asst. Prof., Univ. of Illinois, Urbana, Ill. 362 Mathematics Bldg.

Wilson, W. H. Prof. of Practical Logic, Univ. of Florida, Gainesville, Fla. Box 2227, University Station.

Wine, R. L. Asst. Prof., Washington and Lee Univ., Lexington, Va. Davidson Park Apt., Washington St.

Wing, G. M. Fellow, Cornell Univ., Ithaca, N.Y.

Winger, R. M. Prof., Univ. of Washington, Seattle 5, Wash.

Winston, Dr. Clement. Economic Analyst, Commerce Dept., Washington 25, D.C. 1420 Tuckerman St. N.W., Washington 11.

Winter, Eva P. (Mrs. A. B.). 114 Heatherdown Rd., Decatur, Ga.

Wirth, H. P. Asso. Prof., City Coll., New York 31, N.Y.

Wise, Dr. W. H. Member of Tech. Staff, Bell Telephone Labs., Inc., 463 West St., New York 14, N.Y.

Witmer, E. E. Asst. Prof., Physics Dept., Univ. of Pennsylvania, Philadelphia 4, Pa. Randal Morgan Lab.

Witz, Harold. Instr., Chicago Tech. Coll., Chicago 16, Ill. 749 S. Kostner Ave., Chicago 24.

Wolf, František. Asso. Prof., Univ. of California, Berkeley 4, Calif. Box 66 W.

Wolf, Louise A. Asst. Prof., Univ. of Wisconsin in Milwaukee, Milwaukee 3, Wis. 3700 S. 116th St., Milwaukee 14.

Wolfe, Herbert. Security Analyst, Birnbaum and Co., 60 Broad St., New York 4, N.Y. 755 Ocean Ave., Brooklyn 26, N.Y.

Wolfe, H. E. Asso. Prof., Indiana Univ., Bloomington, Ind. 812 S. Fess St.

Wolfe, J. H. Teaching Fellow, Harvard Univ., Cambridge 38, Mass. 42 Kirkland St.

Wolfe, J. M. Asst. Prof., Brooklyn Coll., Brooklyn 10, N.Y.

Wolfe, R. S. Instr., Northwestern Univ., Evanston, Ill.

Wolfenden, H. H. Consulting Actuary and Stat., P.O. Box 63, Station K, 2384 Yonge St., Toronto 12, Ont., Canada.

Wolfowitz, Jacob. Asso. Prof., Math. Stat. Dept., Columbia Univ., New York 27, N.Y.

Wong, Y. C. Prof., Sun Yat-Sen Univ., Canton, China.

Wong, $\mathbf{Y}$. $\dot{K}$. Asso. Prof., Univ. of North Carolina, Chapel Hill, N.C. Member, Inst. for Advanced Study, Princeton, N.J.

Wood, Frederick. Dean, Univ. of Nevada, Reno, Nev.

Wood, F. E. Asso. Prof., Univ. of Oregon, Eugene, Ore.

Wood, F. L. Instr., State Teachers Coll., Bridgewater, Mass. Box 368, 9 Alsada Rd., Nantasket Beach, Mass.

Wood, Dr. H. A. Senior Project Analytical Eng., Chance Vought Aircraft, Stratford, Conn. 1843 Elm St.

Woodard, D. W. Care of Dr. W. Tate., 5324 S. Park Ave., Chicago 16, I11.

Woodbridge, Dr. Margaret Y. (Mrs. D. E.). Instr., Brooklyn Coll., Brooklyn 10, N.Y. 169 Columbia Heights, Brooklyn 2.

Woodbury, Dr. M. A. Instr., Univ. of Michigan, Ann Arbor, Mich.

Woods, C. L. Dean and Prof., Pacific Union Coll., Angwin, Calif. P.O. Box 235.

Woods, F. S. Prof. Emeritus, Massachusetts Inst. of Tech., Cambridge 39, Mass. 123 Sumner St., Newton Centre 59, Mass.

Woods, L. P. Prof., Northeastern State Coll., Tahlequah, Okla.

Woods, Roscoe. Asso. Prof., State Univ. of Iowa, Iowa City, Iowa. 210 B. Physics Bldg.

Woolson, J. R. Chief, Seismograph Party, United Geophysical Co., Inc., 822 Thompson Bldg., Tulsa 3, Okla.

Worthington, Euphemia R. Asst. Prof., Univ. of California at Los Angeles, Los Angeles 24, Calif.

Wouk, Arthur. Johns Hopkins Univ., Baltimore 18, Md.

Wozencraft, J. G. Univ. of Illinois, Urbana, Ill. 502 W. John St., Champaign, Ill. 
Wray, J. W. Asst., Univ. of Illinois, Urbana, Ill. 604 E. Chalmers St., Champaign, IIl.

Wray, Dr. W. D. Math., Naval Communications Station, Washington 16, D.C. Box 127-B, R.F.D. 1, McLean, Va.

Wrench, Dr. J. W. Math., David Taylor Model Basin, Navy Dept., Washington 7, D.C. 4711 Davenport St. N.W., Washington 16.

Wright, C. B. Prof., East Texas State Teachers Coll., Commerce, Tex. 1603 Bonham St.

Wright, Frances M. Instr., Triple Cities Coll., Syracuse Univ., Endicott, N.Y.

Wright, H. N. Pres., City Coll., New York 31, N.Y.

Wright, J. B. Univ. of Michigan, Ann Arbor, Mich. 2457 Parkwood.

Wright, Martin. Asst. Prof., Univ. of Houston, Houston 4, Tex. 1905 Idylwood Dr., Houston 12.

Wright, R. E. Univ. of Chicago, Chicago 37, I11. 6322 Stony Island Ave.

Wu, S. C. Prof., Hunan Univ., Changsha, Hunan, China.

Wulf, Dr. L. M. Instr., Monmouth Junior Coll., Long Branch, N.J. 123 Washington St.

Wundheiler, A. W. Prof., Mech. Dept., Illinois Inst. of Tech., Chicago 16, Ill.

Wurster, Marie A. Asst. Prof., Temple Univ., Philadelphia 22, Pa. 6635 McCallum St., Philadelphia 19.

Wurtele, Zivia S. (Mrs.). 510 Gayley, Los Angeles 24, Calif.

Wyckoff, J. F. Research Div., Actuarial Dept., Connecticut General Life Ins. Co., Hartford, Conn. $78 \mathrm{~W}$. Cedar St., Newington 11, Conn.

Wylie, C. R. Prof., Univ. of Utah, Salt Lake City 1, Utah.

Wyman, Max. Asst. Prof., Univ. of Alberta, Edmonton, Alta., Canada.

Wynne, W. M. Com., U.S.N., retired. 149 68th St., Brooklyn 20, N.Y.

Wysocki, Theodore. Asst., Univ. of Wisconsin, Madison 6, Wis. 315 N. Brooks, Madison 5.

Yadoff, Dr. O. I. Asso., School of Eng., Columbia Univ., New York 27, N.Y. 27 W. $82 \mathrm{~d}$ St., New York 24.

Yagi, Dr. Fumio. Instr., Univ. of Washington, Seattle 5, Wash. Philosophy Hall.

Yahner, Rev. C. G. Prof., St. Vincent Coll., Latrobe, Pa. St. Vincent Archabbey.

Yancey, L. E. Instr., Morehouse Coll., Atlanta, Ga. 166 Ashby St. S.W.

Yanney, B. F. Prof. Emeritus, Coll. of Wooster, Wooster, Ohio. 354 E. Bowman St.

Yanosik, G. A. Asso. Prof., New York Univ., New York, N.Y. 52 Greenvale Ave., Yonkers 3, N.Y.

Yarbrough, H. M. Prof., Western Kentucky State Coll., Bowling Green, Ky. Route 4.

Yarnell, J. L. Univ. of Kansas, Lawrence, Kan. 1201 Ohio St.

Yarnelle, J. E. Prof., Hanover Coll., Hanover, Ind.

Yarosheski, Marcella. Lib., Archmere Acad., Claymont, Del. 1056 Main St., Dickson City, $\mathrm{Pa}$.

Yeardley, N. P. Fellow, Univ. of Cincinnati, Cincinnati 21, Ohio. 2808 Alice St., Cincinnati 19.

Yeaton, C. H. Prof., Oberlin Coll., Oberlin, Ohio. 189 Forest St.

Yeaton, Dr. Marie J. (Mrs. C. H.). 189 Forest St., Oberlin, Ohio.

Yntema, T. O. Prof., School of Business, Univ. of Chicago, Chicago 37, Ill. 5729 Blackstone Ave.

Yood, Dr. Bertram. Instr., Cornell Univ., Ithaca, N.Y. White Hall.

Young, C. G. Instr., Univ. of Santa Clara, Santa Clara, Calif.

Young, G. S. Asst. Prof., Univ. of Michigan, Ann Arbor, Mich

Young, J. W. Asst. Instr., Univ. of Pennsylvania, Philadelphia 4, Pa. 4033 Spruce St.

Young, J. W. A. Asso. Prof., Univ. of Chicago, Chicago 37, Ill. 5422 Blackstone Ave., Chicago 15.

Young, L. C. Prof., Univ. of Cape Town, Cape Town, South Africa. Lalisa, Talana Rd., Newlands, Cape Town.

Young, Mabel M. Prof. Emeritus, Wellesley Coll., Wellesley 81, Mass. 6 Norfolk Ter.

Young, N. F. Asst. Prof., Emory and Henry Coll., Emory, Va. P.O. Box 51. 
Young, P. M. Asso. Prof., Kansas State Coll. of Agric. and Applied Science, Manhattan, Kan.

Young, R. L. Taft School, Watertown, Conn.

Young, R. W. Instr., Univ. of Florida, Gainesville, Fla. 101 Annis Blvd.

Youngs, J. W. T. Prof., Indiana Univ., Bloomington, Ind.

Yowell, E. I. Prof. Emeritus, Univ. of Cincinnati, Cincinnati 21, Ohio. 3127 Griest Ave., Cincinnati 8.

$\mathrm{Yu}, \mathbf{A}$. T. Fellow, Frits Lab., Lehigh Univ., Bethlehem, $\mathrm{Pa}$.

Zaanen, A. C. Prof., Univ. of Indonesia, Bandoeng, Java.

Zabb, N. J. 210 E. 51 st St., Brooklyn 3, N.Y.

Zabronsky, Herman. Instr., Univ. of Pennsylvania, Philadelphia 4, Pa. 264 Albany Ave., Brooklyn 13, N.Y.

Zadeh, L. A. Instr., Elec. Eng. Dept., Columbia Univ., New York 27, N.Y.

Zanolar, Rev. A. J. Asso. Prof., St. Joseph's Coll., Collegeville, Ind.

Zarantonello, Dr. E. H. 150 Prospect St., Cambridge 39, Mass.

Zariski, Oscar. Prof., Harvard Univ., Cambridge 38, Mass. 7 Avon St.

Zarling, Lillian B. Instr., Extension Center, Univ. of Wisconsin, Green Bay, Wis.

Zassenhaus, Dr. H. J. Dir., Math. Seminar, Univ. of Hamburg, Hamburg, Germany.

Zatzkis, Henry. Instr., Syracuse Univ., Syracuse 10, N.Y.

Zeichner, Arthur. Harvard Univ., Cambridge 38, Mass. Pierce Hall 223.

Zeiders, H. L. Prof., Univ. of Toledo, Toledo, Ohio. Geigertown, $\mathrm{Pa}$.

Zeig, J. J. Tutor, City Coll., New York 31, N.Y. 1750 Grand Ave., New York 53.

Zeigler, R. K. State Univ. of Iowa, Iowa City, Iowa. 1111 Finkbine Park.

Zeldin, S. D. Asso. Prof., Massachusetts Inst. of Tech., Cambridge 39, Mass.

Zelinsky, Dr. Daniel. National Research Fellow, Inst. for Advanced Study, Princeton, N.J.

Zeller, Sister M. Claudia. Asst. Prof., Coll. of St. Francis, Joliet, Ill.

Zemmer, J. L. Asst., Univ. of Wisconsin, Madison 6, Wis. 927 Lake Ct., Madison 5 .

Zettler-Seidel, P. W. Naval Ordnance Lab., White Oak, Silver Spring 19, Md. Bldg. 90.

Zia, J. V. Asst., Univ. of Maryland, College Park, Md.

Ziegler, J. R. Teaching Asst., Univ. of California at Los Angeles, Los Angeles 24, Calif.

Zilber, J. A. Lect., Columbia Univ., New York 27, N.Y. 530 Furnald Hall.

Zilmer, Dr. D. E. Math., Naval Ordnance Test Station, China Lake, Calif. 110-A Mitscher Rd.

Zimmer, J. W. Asst. Prof., Michigan State Coll., East Lansing, Mich. 117 Center St.

Zimmerberg, H. J. Asst. Prof., Rutgers Univ., New Brunswick, N.J.

Zippin, Leo. Asso. Prof., Queens Coll., Flushing, N.Y. 984 E. 27th St., Brooklyn 10, N.Y.

Zobel, Dr. O. J. Member of Tech. Staff, Bell Telephone Labs., Inc., 463 West St., New York 14, N.Y.

Zora, V. A. Chemical Process Eng., Chemical Plants Div., Blaw-Knox Construction Co., 321 Pennsylvania Ave., Pittsburgh, Pa. R.F.D. 2, Wexford, $\mathrm{Pa}$.

Zorn, M. A. Prof., Indiana Univ., Bloomington, Ind. Swain Hall.

Zuckerman, H. S. Asso. Prof., Univ. of Washington, Seattle 5, Wash.

Zulon, J. A. Harvard Univ., Cambridge 38, Mass. 150 Webster Ave.

Zygmund, Antoni. Prof., Üniv. of Chicago, Chicago 37, Ill. Eckhart Hall.

Number of Members, November 1, 1946 (including 211 nominees of Institu-

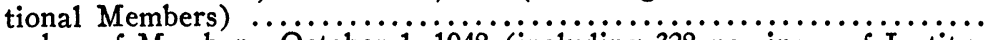

Number of Members, October 1,1948 (including 328 nominees of Institu-

tional Members) $\ldots \ldots \ldots \ldots \ldots \ldots \ldots \ldots \ldots \ldots \ldots \ldots \ldots \ldots \ldots \ldots \ldots, 3805$

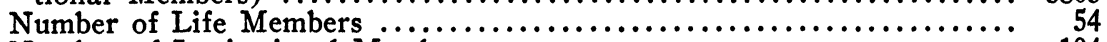

Number of Institutional Members $\ldots \ldots \ldots \ldots \ldots \ldots \ldots \ldots \ldots \ldots \ldots \ldots \ldots \ldots, 104$ 